\title{
The use of charge -charge correlation in impedance measurements: A test of the EPET method
}

Christopher W. Gregory

West Virginia University

Follow this and additional works at: https://researchrepository.wvu.edu/etd

\section{Recommended Citation}

Gregory, Christopher W., "The use of charge -charge correlation in impedance measurements: A test of the EPET method" (2005). Graduate Theses, Dissertations, and Problem Reports. 2326.

https://researchrepository.wvu.edu/etd/2326

This Dissertation is protected by copyright and/or related rights. It has been brought to you by the The Research Repository @ WVU with permission from the rights-holder(s). You are free to use this Dissertation in any way that is permitted by the copyright and related rights legislation that applies to your use. For other uses you must obtain permission from the rights-holder(s) directly, unless additional rights are indicated by a Creative Commons license in the record and/ or on the work itself. This Dissertation has been accepted for inclusion in WVU Graduate Theses, Dissertations, and Problem Reports collection by an authorized administrator of The Research Repository @ WVU.

For more information, please contact researchrepository@mail.wvu.edu. 


\title{
THE USE OF CHARGE-CHARGE CORRELATION IN IMPEDANCE
} MEASUREMENTS: A TEST OF THE EPET METHOD

\author{
by
}

\section{Christopher W. Gregory}

Dissertation submitted to the College of Engineering and Mineral Resources at West Virginia University

in partial fulfillment of the requirements

for the degree of

\author{
Doctor of Philosophy \\ in \\ Electrical Engineering
}

\author{
Approved by \\ Bojan Cukic, Ph.D., Committee Chairperson \\ Wils Cooley, Ph.D. \\ Roy Nutter, Ph.D. \\ Stephanie Schuckers, Ph.D. \\ Raymond Raylman, Ph.D. \\ Donald McRae, Ph.D.
}

Lane Department of Computer Science and Electrical Engineering

$$
\begin{gathered}
\text { Morgantown, West Virginia } \\
2005
\end{gathered}
$$

Keywords: Electrical Impedance Measurement, Boundary Element Method Copyright 2005 Christopher Gregory 


\title{
ABSTRACT \\ THE USE OF CHARGE-CHARGE CORRELATION IN IMPEDANCE MEASUREMENTS: A TEST OF THE EPET METHOD
}

\author{
by Christopher W. Gregory
}

It is well known that biological tissues possess electrical impedance properties that might be useful in medical diagnostics and treatment. Electrical Impedance Tomography (EIT) images internal electrical properties by using numerical methods to solve Laplace's differential equation. The indirect reconstruction method (IRM), a common method in application, predicts internal electrical property distribution by iteratively computing a forward and inverse solution. This approach reduces the non-linear Laplace's equation into a poorly conditioned series of linear equations, which are solved simultaneously. This method suffers from high computational effort and is susceptible to prediction errors that stem from measurement noise.

As an alternative to Laplace's differential equation, this research applies the quasi-static approximation, Dirichlet boundary conditions and a rectangular shaped domain (with corresponding Green's function for Cartesian coordinates) to solve the integral form of Poisson's equation (Green's $2^{\text {nd }}$ identity). The result is the charge-charge correlation method (CCCM), a well-conditioned relationship between charge build-up at internal structures and induced domain boundary charge build-up (which corresponds to measured boundary current). The CCCM is applied in a reconstruction technique called Electrical Property Enhanced Tomography (EPET). While related to the existing impedance imaging methods, EPET does not attempt to create the image with the electrical data but rather adds electrical property information to an existing conventional imaging modality (CT or MI) and, in fact, requires the data from the other modality to locate the position of internal structures in the object. Predicted electrical properties are then superimposed over the a priori structural image to yield the electrical property distribution.

To test the feasibility of the CCCM, experiments using agar media placed in a saline bath were performed. The position, size and conductivity of the agar were varied and the CCCM was applied to predict the conductivities from external boundary current measurements. Predicted conductivities yielded relative errors less than $10 \%$, results that are equal to or better than the IRM. Additionally, CCCM was able to compute these results with a $10^{4}$ improvement in speed over the IRM. 


\section{DEDICATION}

The author wishes to dedicate this work to two separate people, Peter Klein and Maria del Refugio Urita. Pete and I were physics majors and friends at Clarkson University. He was an excellent student who understood that success in life was not limited to just getting good grades. Pete was an outgoing person who was active in many hobbies and helped me break out of my shell. Peter Anton Klein was born on May 2, 1966 in Poughkeepsie, NY and died on September 11, 2001 on the $96^{\text {th }}$ floor of the World Trade Center. His life was senselessly ended by a terrorist attack. The world is a poorer place without him. Rest in peace.

Maria del Refugio is my wife and my partner. This effort has been a long, long journey. She has patiently, perhaps too patiently, waited for me to finish this work. In the course of our marriage she has taught me about sacrifice and love. Maria sacrificed her career to be with me. Together we have mourned when we wanted to start a family and couldn't, and celebrated when we were able to adopt two beautiful children. We are looking forward to new challenges in the future, a future that I know will be full of great adventures and joy. Cuqui, I dedicate this work to you, David and Eileen. Te amo. 


\section{ACKNOWLEDGMENTS}

The author wishes to thank a number of people for their help, guidance, and support throughout the years. First, I want to thank my advisor Dr. Bojan Cukic. He offered advice and guidance from the very beginning and when I was most frustrated, he assured me that I would complete the dissertation. I sincerely thank him for agreeing to be my advisor and committee chair and for his confidence in me. Next, I want to thank Dr. Don McRae. His invaluable technical advice guided me from the Ph.D. proposal to the dissertation defense. He helped me through some difficult times and set me on the right track to complete the job. Finally, his feedback on the dissertation draft allowed me to set forth a brief and concise document. I want to additionally thank the remaining members of the committee, Dr. Wils Cooley, Dr. Roy Nutter, Dr. Stephanie Schuckers, and Dr. Ray Raylman for their feedback throughout the years.

A number of people assisted me while I worked at the University of Wisconsin at Milwaukee. Al Nichols is a superb tool and die maker who fabricated the data acquisition tank. Dr. Dale Buechler, who shared laboratory space with me, has been one of my strongest supporters by listening to my to my ideas, sharing his thoughts, and helping me address issue after issue to complete the experimental work. He sacrificed time with his family and his own sleep to read and critique my entire dissertation. Dr. David Klemer volunteered his time to read and critique my dissertation draft. He helped me keep a schedule and his advice was critical in my finishing the dissertation draft. Lastly, Dr. Susan Cashin was instrumental in conducting the statistical analysis of the experimental data. She met with me numerous times and guided me in understanding how to interpret the results.

I want to thank my family. Thanks to my parents for raising me to value education. My mother always encouraged me, even when I was discouraged. My father has been an advisor and collaborator for more than a decade and together we developed and advanced the concepts contained in this dissertation. Thanks to my four brothers for continuously encouraging me to pursue my Ph.D. Finally, I am eternally indebted to my wife, Maria del Refugio. She has loved me and encouraged me even though she has endured many years of uncertainty and financial strain. She was a successful dentist in her home country of Mexico and left her practice behind to be with me. Love is when one person sacrifices for the other. I know my wife loves me. Now it's my turn to sacrifice for her. 


\section{TABLE OF CONTENTS}

ABSTRACT _ ii

DEDICATION _ iii

ACKNOWLEDGMENTS__ iv

TABLE OF CONTENTS__ V

LIST OF FIGURES__ viii

LIST OF TABLES__ ix

1 INTRODUCTION _ 1

1.1 Electrical Impedance Tomography___ 1

1.2 Issues Limiting EIT__ 2

1.3 Electrical Property Enhanced Tomography: A New Approach to EIT _ 3

1.4 Experimental Overview __ 4

1.5 Dissertation Outline __ 4

2 BACKGROUND AND LITERATURE SURVEY___ 6

2.1 Biological Tissue Electrical Property Characteristics__ 6

2.1.1 Dispersion Regions____ 7

2.1.2 Characterizing Tissue with Conductivity and Relative Permittivity __ 11

2.1.3 High Relative Permittivity Values for Biological Tissues ___ 12

2.1.4 Differentiating Normal and Abnormal (cancerous) Tissue __ 13

2.1.5 Characteristics of Breast Cancer Lesions __ 13

2.1.6 Temperature Dependence of Tissue Conductivity and Relative Permittivity _ 15

2.1.7 Anisotropic Dependence on Tissue Conductivity and Relative Permittivity _ _ 15

2.2 Medical Applications Using Biological Electrical Properties ___ 16

2.2.1 Breast Cancer Screening____ 16

2.3 The Electrical Impedance Tomography __ 18

2.4 Image Reconstruction Methods in EIT _ 20

2.4.1 Early EIT Research____ 21

2.4.2 Reviews of EIT__ 22

2.4.3 Direct Method__ 22

2.4.4 Indirect Method__ 22

$3 \quad$ THEORY 29

3.1 Charge-charge Correlation __ 31

3.1.1 Simplifying the Problem 32

3.1.2 Reducing the Expression to Charge and Potential __ 33

3.1.3 Discretizing the Formulation __ 34

3.1.4 Applying the Green's Function for Cartesian Coordinates___ 35

3.1.5 Using Sine Transforms to Produce the Charge-charge Correlation Relationship 36 
3.2 Relating Internal Charge to Electrical Property Values

3.3 Electrical Property Enhanced Tomography __ 38

3.3.1 EPET Forward Solution __ 41

3.3.2 EPET Inverse Solution __ 41

4 MATERIALS AND METHODS _ 43

4.1 Description of Experiments __ 43

4.1.1 Two-Media Experiments 433

4.1.2 Three Media Experiments___ 46

4.2 Numerical Methods_ 48

4.2.1 Application of Charge-charge Correlation Method __ 48

4.2.2 Application of the Indirect Reconstruction Method __ 51

4.3 Sample Preparation

4.3.1 Agar__ 55

4.3.2 Liver 55

4.3.3 Oatmeal__ 55

4.4 Determining Internal Boundary Locations a priori _ 56

4.4.1 Templates for Two Media Experiments ___ 56

4.4.2 Position Spacers for Three Media Experiments with Agar _ 56

4.4.3 Digital Images for Three Media Experiments with Liver and Oatmeal ___ 57

4.5 Experimental Procedures__ 58

4.5.1 Two Media Experiments___ 58

4.5.2 Three Media Experiments with Agar __ 58

4.5.3 Three Media Experiments with Liver and Oatmeal ___ 59

4.6 Experimental Set-up _ 59

5 RESULTS AND DISCUSSION — 66

5.1 Raw Experimental Data: Description and Characterization __ 66

5.1.1 Typical Raw Current Pattern ___ 66

5.1.2 Current Pattern Dependence on Position, Size and Contrast ___ 67

5.1.3 Quantifying the Similarity in Current Pattern Shape __ 70

5.1.4 Examining Current Magnitude Dependence on Contrast___ 72

$5.2 \quad$ Forward Solution Results__ 75

5.2.1 Comparing Measured and Predicted Current Patterns __ 75

5.2.2 Estimating Limits on the Forward Solution ___ 77

5.3 Inverse Solution Results

5.3.1 Two Media Results: Predicted Contrast vs. Depth___ 82

5.3.2 Two Media Results: Predicted Contrast vs. Size ___ 87

5.3.3 Three Media Results Using Agar __ 89

5.3.4 Three Media Results Using Liver and Oatmeal ___ 92

5.4 Charge-charge Correlation vs. Indirect Reconstruction Method _ 99

6 SUMMARY AND FUTURE WORK__ 102 
6.1 The Proposed Solution 102

6.2 Conclusions _ 103

6.3 Future Work 105

APPENDIX A: EPET Related Publications__ 107

APPENDIX B: Using FDM to Model the Uniform Case Potential___ 113

APPENDIX C: Observed Current Pattern Dependence on Lateral Position __ 116

CURRICULUM VITAE__ 118

REFERENCES 121 


\section{LIST OF FIGURES}

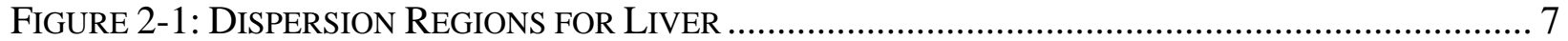

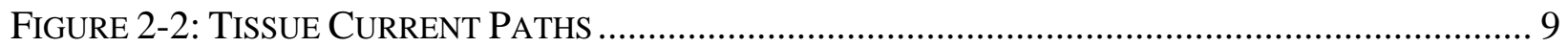

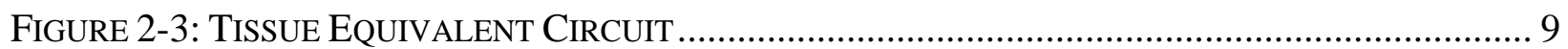

FigURE 2-4: FREQUENCY DEPENDENCE OF TISSUE CONDUCTIVITIES ........................................ 10

Figure 2-5: FreQuency DePENDENCE Of TISSUE RELATIVE PERMITTIVITIES .......................... 11

FIGURE 2-6: FREQUENCY DEPENDENCE OF BREAST CANCER CONDUCTIVITY ............................. 14

FIGURE 2-7: FREQUENCY DEPENDENCE OF BREAST CANCER RELATIVE PERMITTIVITY................. 14

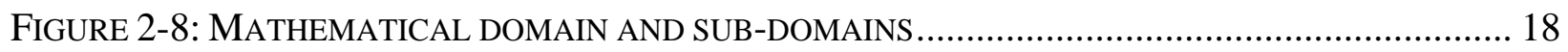

FIGURE 2-9: FUNCTIONAL FLOW CHART OF THE INDIRECT RECONSTRUCTION METHOD ............... 23

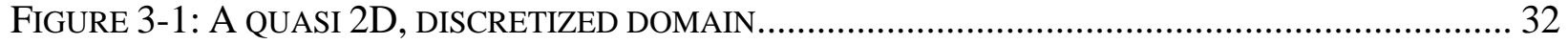

FIGURE 3-2: REPRESENTATION OF ELECTRICAL PROPERTIES AT INTERNAL BOUNDARIES ............... 37

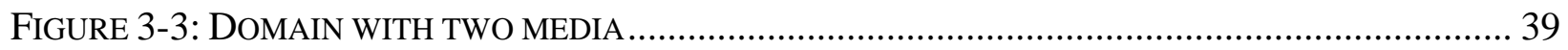

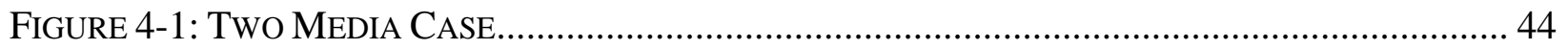

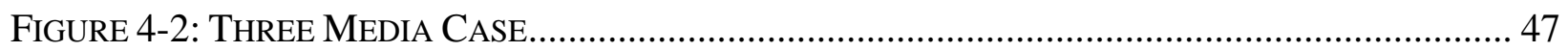

FIGURE 4-3: THE TWO MEDIA CASE WITH CIRCULAR SHAPED $2^{\text {ND }}$ MEDIUM .................................. 50

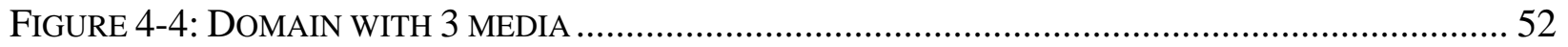

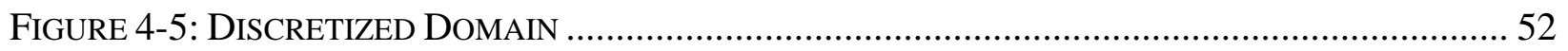

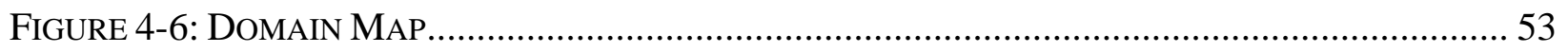

FIGURE 4-7: CLOSE-UP VIEW OF POTENTIAL VERTICES AND ELECTRICAL PROPERTY ELEMENTS .... 53

FIGURE 4-8: ORIGINAL PHOTO OF THE LIVER AND OATMEAL SAMPLE PLACE IN SAMPLE HOLDER .. 57

FIGURE 4-9: FINAL ELECTRICAL PROPERTY MAP FOR FDM PROCESSING .................................. 57

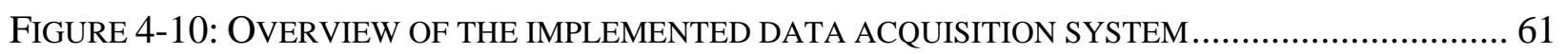

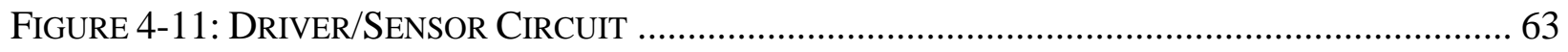

FIGURE 4-12: (ABOVE) SIDE VIEW AND (BELOW) TOP VIEW OF THE DATA ACQUISITION TANK ...... 65

FIGURE 5-1: I I RELATIVE VS. ELECTRODE NUMBER: THE REFERENCE CASE ....................................... 67

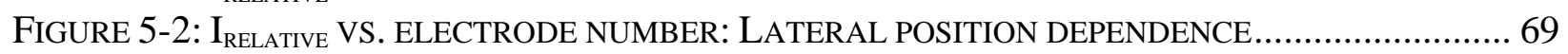

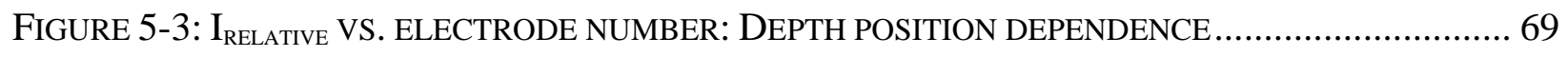

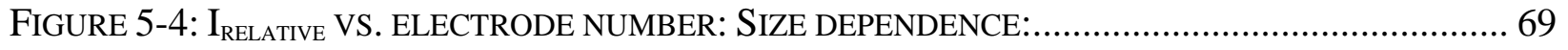

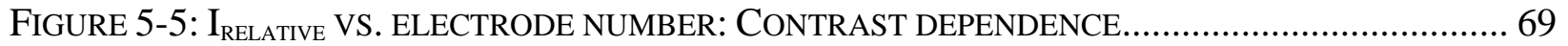

FIGURE 5-6: EXPERIMENTAL AND PREDICTED SCALING FACTOR RESULTS: $\xi$ VS. $\kappa_{21} \ldots \ldots \ldots \ldots \ldots \ldots . . . . . . . .73$

FIGURE 5-7: COEFFICIENT OF VARIANCE VS. SCALING FACTOR FOR EXPERIMENTAL RESULTS........ 75

FIGURE 5-8: I IELATIVE VS. ELECTRODE NUMBER: REFERENCE CASE MEASURED AND PREDICTED...... 76

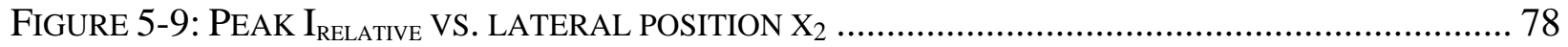

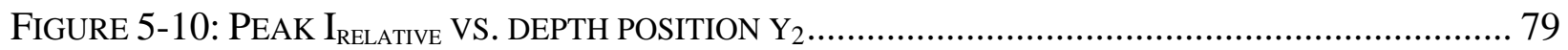

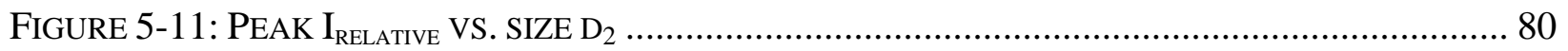

FIGURE 5-12: DETERMINING MINIMUM SIZE AND CONTRAST DETECTABILITY ............................ 81

FIGURE 5-13: PREDICTED CONTRAST VS. DEPTH: THE 2 MEDIA CASE ....................................... 83

FIGURE 5-14: PREDICTED CONTRAST UNCERTAINTIES AS A FUNCTION OF SCALING FACTOR .......... 85

FIGURE 5-15: PREDICTED CONTRAST VS. SIZE: THE 2 MEDIA CASE ......................................... 88

FIGURE 5-16: PREDICTED CONTRAST VS. DEPTH: THE 3 MEDIA CASE ......................................... 91

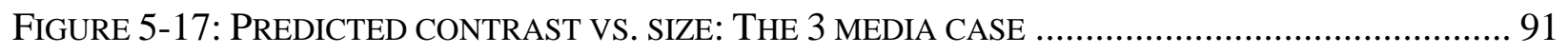

FIGURE 5-18: PREDICTED LIVER CONDUCTIVITY VS. TIME....................................................... 95

FIGURE 5-19: PREDICTED LIVER RELATIVE PERMITTIVITY VS. TIME .......................................... 95

FIGURE 5-20: PREDICTED OATMEAL CONDUCTIVITY VS. TIME ............................................. 97 


\section{LIST OF TABLES}

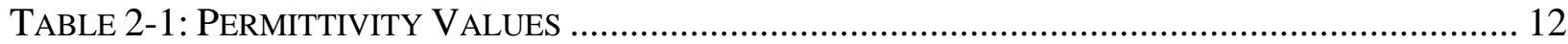

TABle 2-2: Boundary Conditions MATRIX FOR THE Direct ProbleM.................................. 20

TABLE 4-1: PARAMETERS USED EXPERIMENTAL GROUPS 1 THROUGH 6 ........................................45

TABLE 4-2: PARAMETERS USED EXPERIMENTAL GROUPS 7 AND 8 ............................................ 48

TABLE 5-1: POSITION, SIZE, AND CONTRAST VALUES PRESENTED IN FIGURES 5-2 THROUGH 5.5 .. 68

TABLE 5-2: CROSS-CORRELATION COEFFICIENTS AS A FUNCTION OF DEPTH FOR MEDIUM 2 .......... 71

TABLE 5-3: CROSS-CORRELATION COEFFICIENTS AS A FUNCTION OF SIZE FOR MEDIUM 2 .............. 71

TABLE 5-4: CROSS-CORRELATION COEFFICIENTS AS A FUNCTION OF CONTRAST FOR MEDIUM $2 \ldots . .72$

TABLE 5-5: ERROR IN PREDICTED CONTRAST FOR VARIOUS DEPTHS: THE 2 MEDIA CASE ............... 86

TABLE 5-6: ERROR IN PREDICTED CONTRAST FOR VARIOUS SIZES: THE 2 MEDIA CASE ................. 87

TABLE 5-7: ERROR IN PREDICTED CONTRAST FOR VARIOUS DEPTHS: THE 3 MEDIA CASE ............... 90

TABLE 5-8: ERROR IN PREDICTED CONTRAST FOR VARIOUS SIZES: THE 3 MEDIA CASE .................. 90

TABLE 5-9: RELATIVE STANDARD ERROR OF THE ESTIMATE FOR LIVER AND OATMEAL................93

TABLE 5-10: PREDICTED CONTRAST RESULTS USING IRM AND CCCM .................................. 100

TABLE 5-11: COMPARING RELATIVE ERRORS AND COMPUTATION TIMES FOR IRM AND CCCM.. 101 


\section{INTRODUCTION}

Biological tissues possess complex impedance properties (collectively described by conductivity, $\sigma$, and relative permittivity, $\varepsilon_{\mathrm{r}}$ ) that exhibit frequency dependent characteristics when excited with time-varying currents. In general, over the frequency range from 0 to $10^{9} \mathrm{~Hz}$, impedance magnitude decreases with increasing frequency and phase shift is negative with three local phase minima corresponding to the $\alpha, \beta$, and $\gamma$ dispersion regions. Within the $\beta$-dispersion region (1 kHz to $100 \mathrm{MHz}$ ), conductivity and relative permittivity properties differ from tissue to tissue, permitting identification and differentiation. Furthermore, within a given tissue, electrical properties differ between normal and abnormal tissue. Studies of breast cancer tissue, for example, have determined that the conductivity and relative permittivity at the center of a tumor are greater in magnitude than the surrounding normal tissue by one to two orders of magnitude [1].

Exploiting these electrical properties in medical applications is very appealing. Imaging devices based on electrical properties would not expose patients to harmful ionizing radiation from X-rays, are relatively inexpensive to produce because of the availability of low-cost components, and could be made portable for use in small office or remote settings. Researchers have investigated applications for breast cancer screening, monitoring the gastrointestinal system, monitoring and screening the pulmonary system, and monitoring brain function, to name a few [18].

\subsection{Electrical Impedance Tomography}

Electrical Impedance Tomography (EIT) is a modality capable of imaging the electrical property distribution within a tissue medium. The electrical property distribution has been traditionally determined by solving Laplace's equation $[18,74]$ using appropriate boundary conditions and measurements of voltage and/or current:

$$
\nabla \cdot\left(\left(\sigma+j \omega \varepsilon_{0} \varepsilon_{r}\right) \nabla \Phi\right)=0
$$

where $\nabla$ is the gradient operator

$\sigma$ is the conductivity

$\omega$ is the radial frequency

$\varepsilon_{0}$ is the relative permittivity in free space

$\varepsilon_{\mathrm{r}}$ is the relative permittivity

$\Phi$ is the potential 
The solution of this equation is non-trivial and practical applications require that EIT employ numerical methods. The direct method computes a solution in a single step with no intermediate computations. The indirect method performs a two-step process iteratively until an arbitrary termination criterion is met. Yorkey [2] compared direct and indirect methods and showed that the indirect method always converges and produces results with minimum error. Consequently, this research focuses on the indirect method.

A common numerical method, the indirect reconstruction method, is computed iteratively in two parts, the forward and inverse solutions. The forward solution combines an estimate of the electrical property distribution with boundary conditions (e.g., voltage), to produce a prediction of the measured response (e.g., current). Typically, finite-element methods (FEM) or finite-difference methods (FDM) are employed. The inverse solution computes a least squares error estimate (LSEE) from the predicted and measured response and then applies the NewtonRaphson method to compute a correction to the electrical property estimate. The forward solution and inverse solution are repeated until the LSEE reaches a termination threshold. At this point, the estimated conductivity and relative permittivity distribution are presumed to be optimal.

\subsection{Issues Limiting EIT}

Image reconstruction in EIT suffers from two limiting factors: a high computational effort and the susceptibility to relatively large errors in estimating the electrical property distribution resulting from even relatively low noise in measured electrical data (voltages and currents). The high computation time is a result of the computationally expensive numerical methods used in the forward solution. These methods (FEM and FDM) discretize the domain and convert the non-linear Poisson's equation (1-1) into a series of $\mathrm{N}$ linear equations. The computational demand of these methods increases as the order of $\mathrm{N}^{3}$. Finer discretization (i.e., finer resolution of the estimated electrical property distribution) increases the number of computations and computation time dramatically.

The second issue, the susceptibility of the estimates to significant error caused by relatively low noise in measured electrical data (voltages and currents), is traced to the NewtonRaphson method used in the inverse solution. The N-R method requires computation of an illconditioned matrix inversion. The problem is ill-posed and results in unstable matrix inversions. 
Implementing regularization techniques and using a priori anatomical information (from CT or MRI images) are two common techniques used to address this problem. Regularization has helped reduce instability of the matrix inversion, but it does not eliminate the ill-posed nature of the problem. This research presents an alternative approach that poses the problem in a more computationally robust manner.

\subsection{Electrical Property Enhanced Tomography: A New Approach to EIT}

The new approach, called Electrical Property Enhanced Tomography (EPET) was first introduced by Gregory, et al [3] and later extended by Gregory and Gregory [4] and examines

the internal distribution of electrical properties from a Green's $2^{\text {nd }}$ identity perspective [74]. The approach applies a quasi-static approximation for frequencies below $1 \mathrm{MHz}$ and notes that instantaneous (static) charge accumulates only at internal dielectric boundaries within the media under investigation. The Green's second identity for Dirichlet boundary conditions (equation (1-2)), uses the Green's function $\left(G_{D}\right)$ to relate a charge distribution in the interior, $\rho\left(\mathbf{r}^{\prime}\right)$, and the potential on the domain boundary, $\Phi_{\mathrm{S}}\left(\mathbf{r}^{\prime}\right)$, to the potential at any point in the domain, $\Phi(\mathbf{r})$,

$$
\Phi(\mathbf{r})=\frac{1}{4 \pi \varepsilon_{0}}\left[\int_{V} \rho\left(\mathbf{r}^{\prime}\right) G_{D}\left(\mathbf{r}, \mathbf{r}^{\prime}\right) d^{3} x^{\prime}-\varepsilon_{0} \oint_{S} \Phi\left(\mathbf{r}^{\prime}\right) \frac{\partial G_{D}}{\partial n^{\prime}} d a^{\prime}\right]
$$

The work by Gregory and Gregory [4] outlined a derivation of equation (1-2) accompanied by an appropriate selection of the Green's function, $\mathrm{G}_{\mathrm{D}}$, for Cartesian coordinates. This derivation led to a relationship between the interior charge and external charge build-up on the domain boundary that, by the law of conservation of charge, relates measured boundary current to the internal charge buildup. This relationship is referred to as the charge-charge correlation method and has a matrix representation relating interior to boundary charge. From a computational perspective, performing this matrix manipulation is far less costly than either the FEM or FDM.

The method outlined in [4] forms the theoretical basis for the EPET reconstruction technique. While related to the existing impedance imaging methods, the technique differs in a number of substantive ways, EPET does not attempt to create the image with the electrical data but rather adds electrical property information to the existing modality and, in fact, requires the 
data from the other modality to locate the position of internal structures in the object. EPET uses a priori structural information to locate boundaries between different materials within an object under investigation. This structural information reduces the number of electrical property values needed to be reconstructed. It also provides necessary data for use in the charge-charge correlation relationship in the estimation of those electrical properties. The resulting estimates are superimposed over the a priori structural image (such as a CT image) to produce the 'reconstructed' impedance image. The EPET method avoids the high computational expense associated with the finite element and finite difference methods, as well as the ill-posed nature of the Newton-Raphson technique.

\subsection{Experimental Overview}

A number of experiments were performed to test and validate the EPET method. These experiments used media of varying types to estimate the dependence of solution accuracy on position, size, and electrical property magnitude. Experiments were conducted using metallic rods and agar doped with salt; these media possess purely conductive electrical properties. The agar doped with salt is prepared with a specific conductivity, while the metallic objects test the behavior of the method at extreme limits. Experiments with complex impedances were conducted using a three-media domain composed of a saline solution, calves liver, and an object embedded in the liver composed of a mixture of oatmeal, salt and water. The advantage of using liver is that it is a biological medium. These experiments will demonstrate that the EPET method produces repeatable results that are consistently more accurate, less error prone, and far less computationally demanding than the classical indirect reconstruction method.

\subsection{Dissertation Outline}

The remainder of the dissertation is divided into five chapters: Background and Literature Survey, Theory, Materials and Methods, Results and Discussion, and finally a Summary and Future Work chapter.

The Background and Literature Survey, Chapter 2, includes a brief history of early research into the electrical characteristics of biological media, an overview of the electrical properties of these media, and a brief survey of medical applications based on these property characteristics. The chapter then narrows its focus to Electrical Impedance Tomography, an 
imaging modality for obtaining the electrical property distribution. This discussion describes the problem in mathematical terms and describes numerical methods used to render impedance images. The limitations of EIT are discussed in this chapter.

The Theory, Chapter 3, introduces the charge-charge correlation method. The method is then applied to the electrical impedance problem using a priori structural information. This application divides the problem into forward and inverse solution, which are derived from first principles.

The Materials and Methods, Chapter 4, describes experiments using two- and threemedia. The media are composed of either purely resistive materials (saline solutions, agar, oatmeal mixtures, and conducting objects) or materials with complex impedances (liver). The chapter covers experimental procedures, specific numerical methods, sample preparation, and a description of the experimental apparatus.

The Results and Discussion, Chapter 5, presents the experimental results in three sections. The first section examines the characteristics of the observed electrical measurements (current patterns) as a function of the size, position, and electrical properties for two media experiments. Conclusions drawn from these observations are used to support the underlying assumptions made in the development of the charge-charge correlation method. The second section examines the accuracy (fidelity) of the forward solution in the prediction of current patterns, as compared to measured data. This section examines only two-media experiments with metallic objects. The size and depth of the objects are varied to determine limits of the methods. The final section examines the accuracy of the inverse solution in the prediction of electrical properties of a medium. This section presents experimental results for two-media and three-media experiments using saline, agar and metallic objects, as well as three-media experiments using saline, liver and oatmeal mixtures. Experiments using agar tested the accuracy as object position and size were varied, while experiments using liver tested accuracy of predicting results under complex, but more practically relevant, conditions.

The Summary and Future Work, Chapter 6, is the final chapter. It summarizes the results and the difficulties that were encountered and gives recommendations for future work. 


\section{BACKGROUND AND LITERATURE SURVEY}

Early research involving the electrical properties of biological properties extends back more than one century. Stewart, an early pioneer in the study of blood, observed that the conductivity of blood is similar to that of salt solutions. He demonstrated that injecting salt solutions into the circulatory system alters blood conductivity, a technique to study the circulation time of blood through organs [5] and the output of the heart [6]. Höber demonstrated that the cell membrane is involved in the electrical properties of cells and tissues. He studied the passage of alternating current through cells and determined that the cell membrane limits current at low frequency but at high frequencies current passes through the cell membrane [7]. Crile, et al., observed that healthy and diseased tissue have different conductivities [8] and Fricke and Morse observed that cancerous breast tissue has a higher capacitance than healthy breast tissue [9]. Finally, between 1928 and 1944, Cole, et al published results from a series of experiments that examined biological impedance. Their investigations produced an equivalent circuit to model biological impedance behavior and resulted in a graphical description for the vector impedance and the modulus $[10,11]$.

This work led to research to characterize and quantify the electrical properties of different tissues. Early in his career, H. P. Schwan, a noted figure in the field of bioimpedance phenomena, published results on the relaxation phenomena in tissues [12]. Some 30 years later, Foster and Schwan published a review article on the same topic [13]. Geddes and Baker published a compendium of the specific resistance of biological media in 1967 [14]. More recently, Gabriel, et al., compiled the conductivity and dielectric properties of various tissues for frequencies ranging from $10 \mathrm{~Hz}$ to $10^{9} \mathrm{~Hz}$ [15]. Their work also produced models of the equivalent electrical circuits [16].

This chapter examines the electrical properties of biological tissue, surveys various medical applications based on those properties, introduces electrical impedance tomography and finally describes various methods for generating impedance images.

\subsection{Biological Tissue Electrical Property Characteristics}

Biological tissues injected with alternating currents exhibit frequency dependent impedance characteristics. These impedance characteristics are complex (i.e., they possess both 
a real and an imaginary part) and vary as a function of frequency. Figure 2-1 presents impedance magnitude and phase values for liver over the frequency range 10 to $10^{9} \mathrm{~Hz}$, computed from Gabriel, et al. [16, 17]. In general, biological tissues have decreasing impedance magnitude with increasing frequency, three phase local minima, and a negative impedance phase (implying capacitive properties).

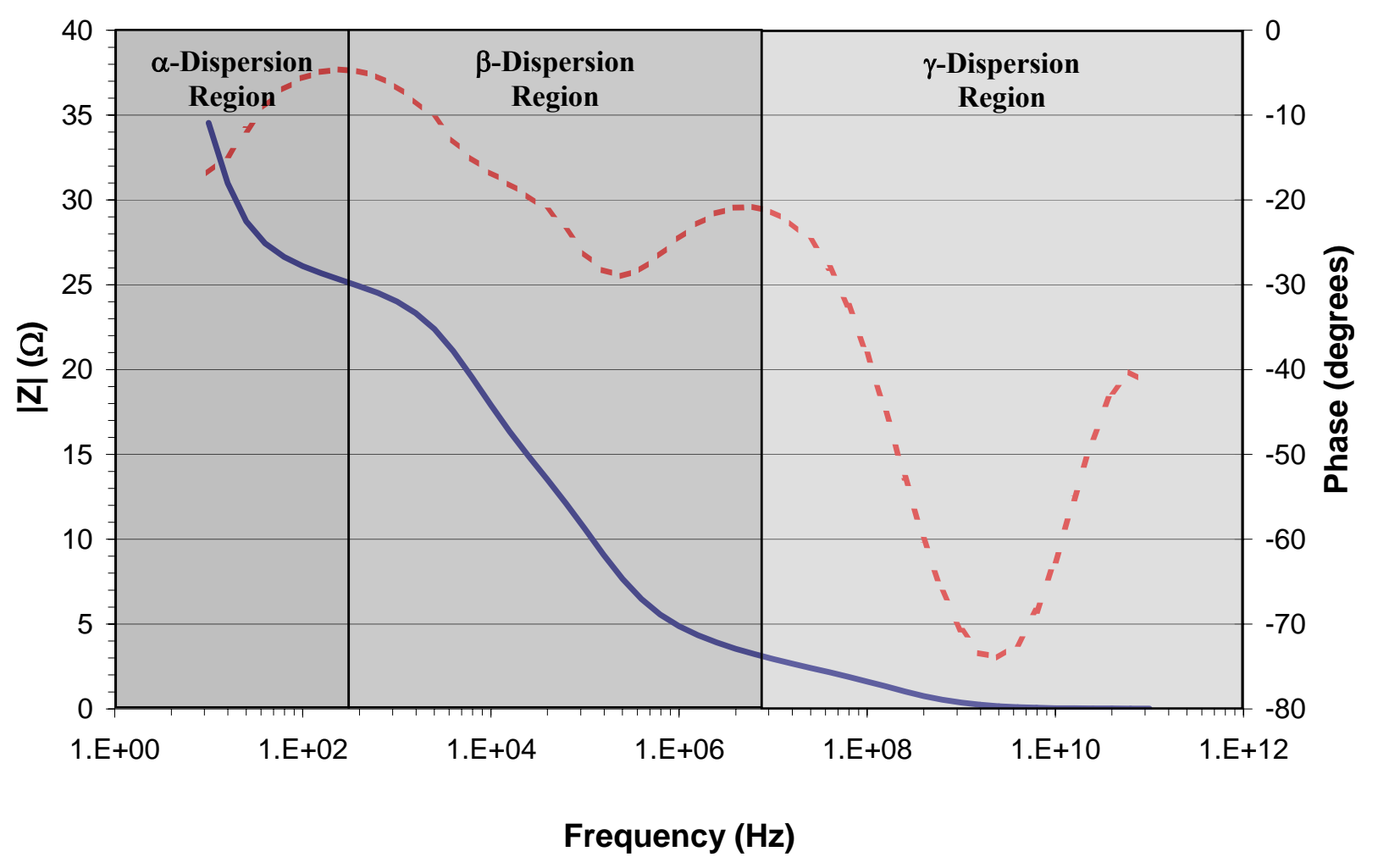

Figure 2-1: Dispersion Regions for Liver

Liver impedance magnitude and phase for frequencies ranging from $10 \mathrm{~Hz}$ to $10 \mathrm{GHz}$ computed for a geometric factor of 1.0 [1/m]. Adapted from Gabriel, et al. [16].

\subsubsection{Dispersion Regions}

The three phase minima correspond to the $\alpha, \beta$, and $\gamma$ dispersion regions. At very low frequencies ( $\mathrm{f}<1 \mathrm{kHz}$ ) $\alpha$-dispersion dominates, attributed to electrochemical polarizations and an ionic atmosphere surrounding the cells, conditions that produce very large relative 
permittivity values $[18,19]$. At frequencies between $1 \mathrm{kHz}$ and $100 \mathrm{MHz} \beta$-dispersion dominates, attributed to Maxwell-Wagner structural relaxation and the cell membrane capacitive response. Cell membrane degradation resulting from cellular death greatly reduces the presence of this dispersion region [18, 19], an important effect exploited in biomedical diagnostic instrumentation based on measurements of tissue impedance. At frequencies greater than 100 $\mathrm{MHz} \gamma$-dispersion dominates, caused by the relaxation of water molecules (Debye effect) [18, 19, 20]. All three of these dispersions generally depend on cellular quantity, shape, and orientation, as well as the tissue chemical composition (i.e., the composition and ionic concentrations of interstitial space and cytoplasm) $[18,19]$. Research described in this dissertation is restricted to frequencies in the $\beta$ dispersion region because of the great interest in using impedance measurements in this frequency range for imaging instrumentation.

Figure 2-2 (adapted from Webster [21]) presents an example of a tissue cross-section at the cellular level, illustrating the behavior of electric current flow across tissue transitions in the $\beta$-dispersion frequency range. The figure illustrates a cell enclosed by its lipid bi-layer membrane and filled with intracellular fluid, surrounded by extra-cellular fluid. An applied electric field oriented from top to bottom of the figure induces an electrical current, which flows through the local cellular environment.

At the low end of the $\beta$-dispersion frequency range ( $\mathrm{f} \cong 1 \mathrm{kHz}$ ), the applied electric field (E-field) induces a charge on the cell membrane, which charges and discharges fully during each cycle of the alternating externally-applied E-field. When fully charged, no current flows into the interior of the cell, resulting in a current flow mainly in the extra-cellular fluid (illustrated as the dashed current flow in the figure). Impedance measurements are almost entirely resistive and reflect only the extra-cellular impedance [18].

Increasing the applied frequency results in incomplete charging of the cell membrane, leading to inter-cellular current flow. The measured impedance is influenced by the cell membrane (which is highly resistive as a result of its nonpolar lipid core), the intra-cellular fluid (highly conductive), and the capacitive nature of the cell membrane (resulting from the phenomenon of charge accumulation at the membrane interface). Accordingly, measured impedance is both resistive and reactive [18]. 


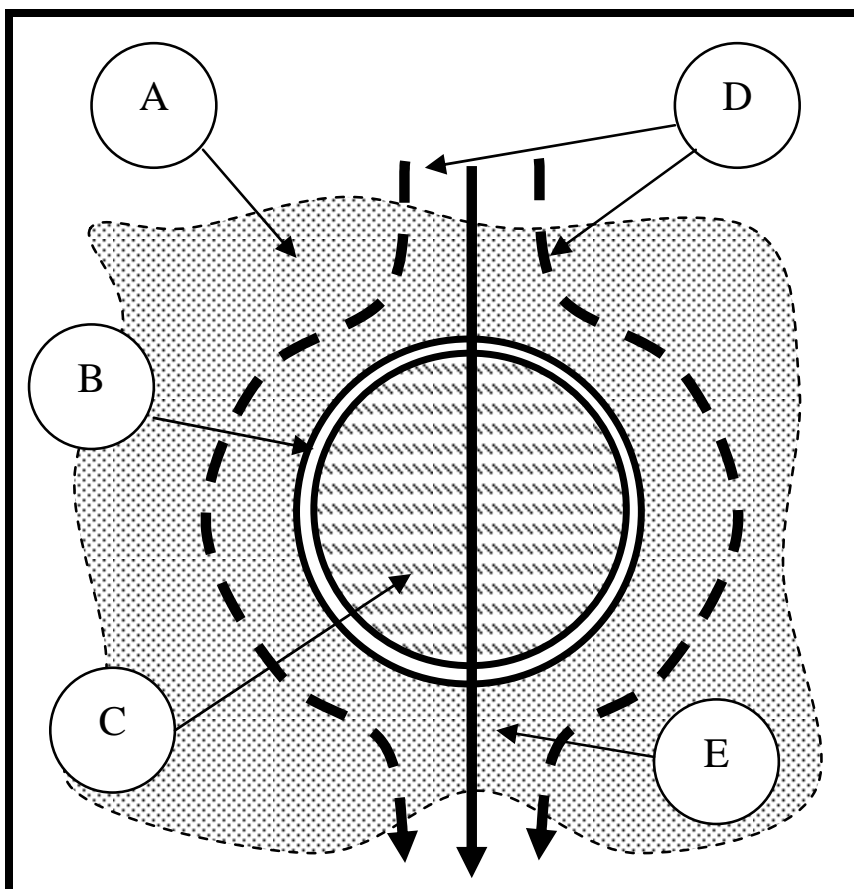
A: Extra-cellular fluid
B: Cell Membrane
C: Intra-cellular fluid
D: Low frequency current path
E: High frequency current path

Figure 2-2: Tissue Current Paths

Illustration of a basic cell with intra- and extracellular fluid as well as a cell membrane.

Current paths at low and high frequencies are illustrated. (adapted from Webster [21])

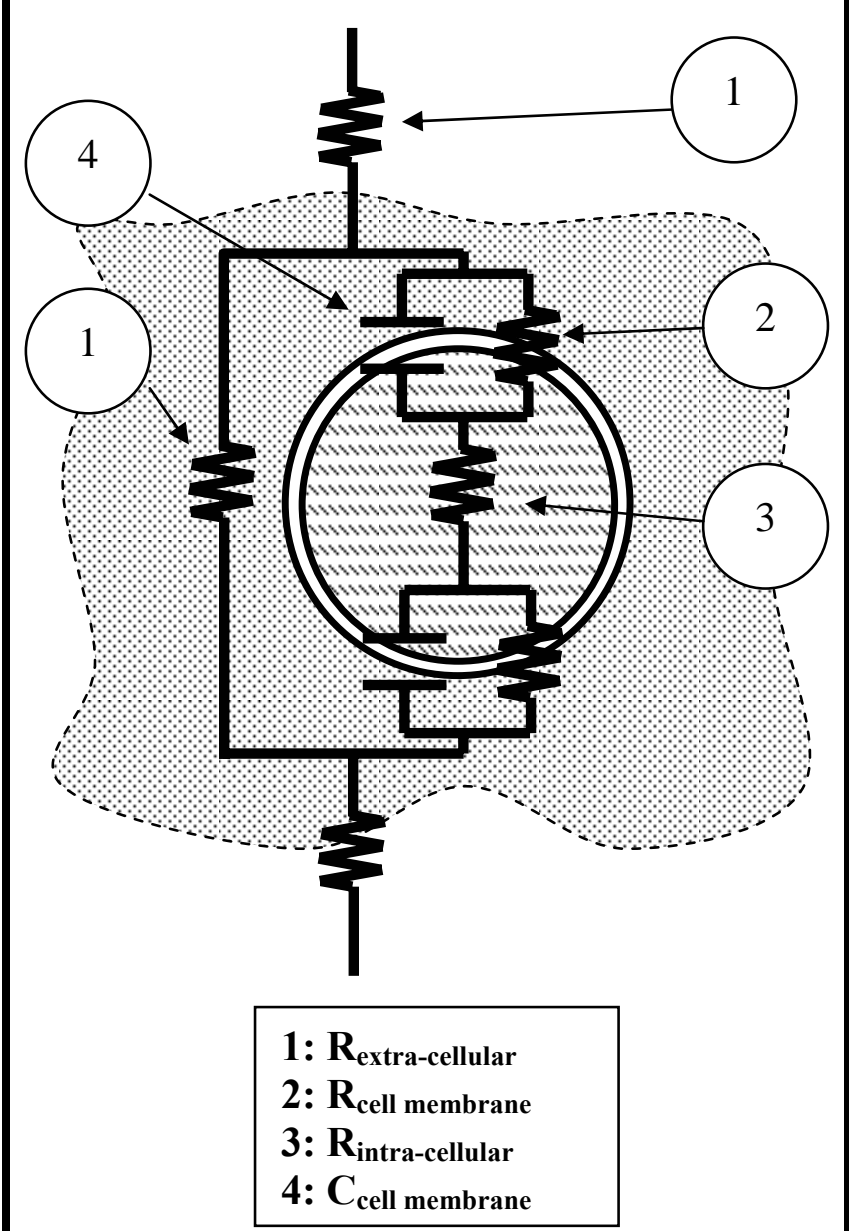

Figure 2-3: Tissue Equivalent Circuit An equivalent circuit, adapted from Webster [21], models the intra- and extra-cellular fluid with resistors and the cell membrane with a parallel resistor/capacitor circuit.

At the upper end of the $\beta$-dispersion frequency range ( $\cong 10 \mathrm{MHz}$ ), charge has

insufficient time to accumulate on the cell membrane. Current flows freely and penetrates the cells easily, resulting in a nearly purely resistive impedance component. This is illustrated as the solid line current flowing directly through the cell in Figure 2-2. 


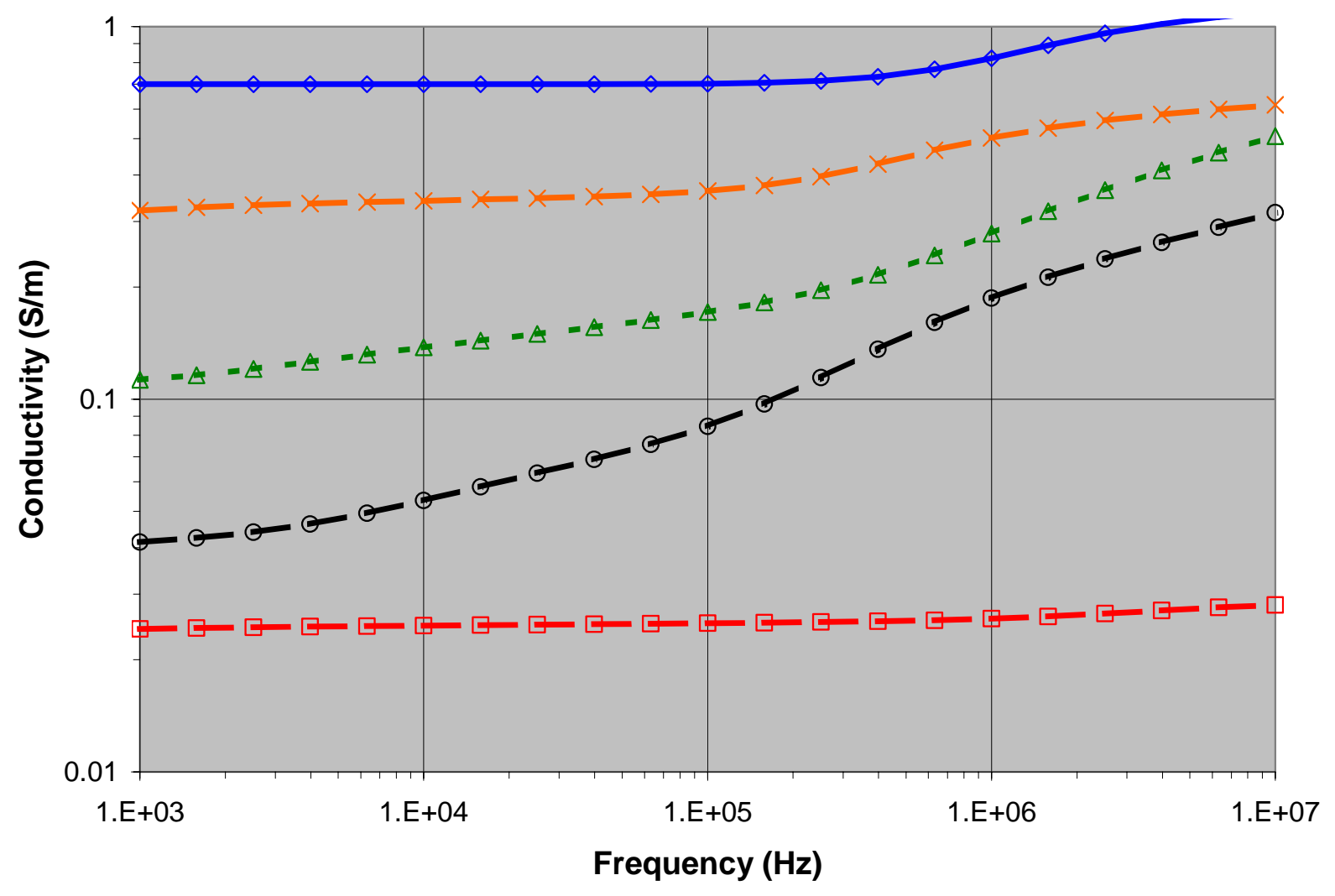

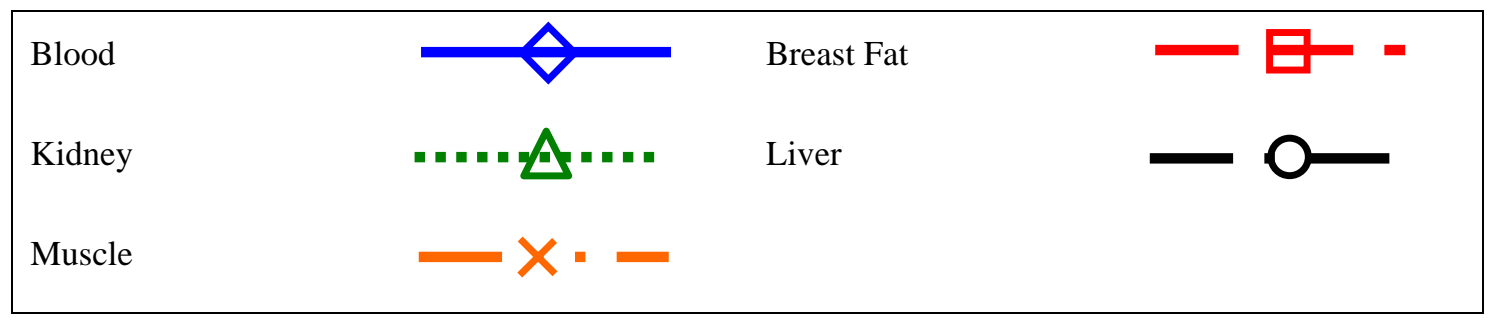

Figure 2-4: Frequency Dependence of Tissue Conductivities

Conductivity values for various biological tissues over the frequency range $1 \mathrm{kHz}$ to $10 \mathrm{MHz}$ (from Gabriel, et al. [16]).

Figure 2-3 presents an equivalent circuit that models the impedance behavior of the cell.

The circuit is composed of 4 different elements: a resistor, $\mathbf{R}_{\text {extra-cellular, associated with the extra- }}$

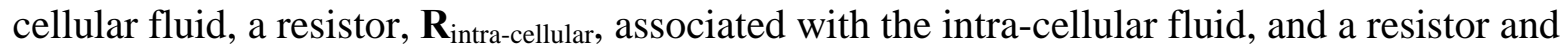

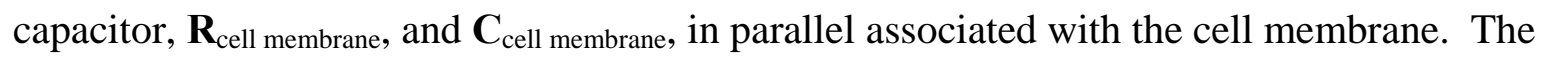
resistive and capacitive characteristics of cells on a microscopic level are also observed on a macroscopic tissue level, and are described by tissue conductivity and relative permittivity, respectively. 


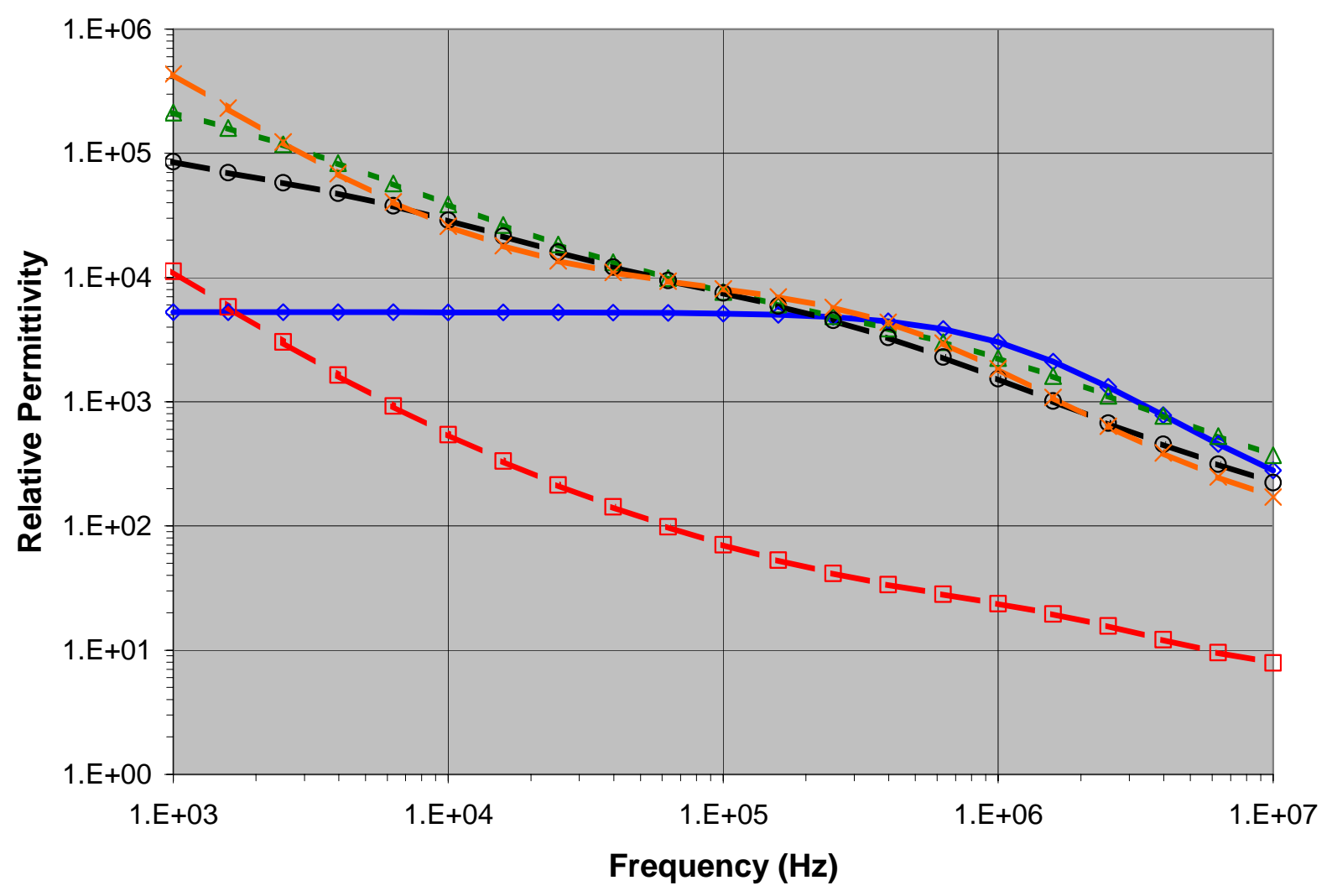

\begin{tabular}{|c|c|c|c|}
\hline Blood & & Breast Fat & E \\
\hline Kidney & 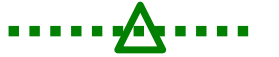 & Liver & E \\
\hline Muscle & X. & & \\
\hline
\end{tabular}

Figure 2-5: Frequency Dependence of Tissue Relative Permittivities

Relative permittivity values for various biological tissues over the frequency range $1 \mathrm{kHz}$ to 10 $\mathrm{MHz}$ (from Gabriel, et al. [16]).

\subsubsection{Characterizing Tissue with Conductivity and Relative Permittivity}

Figure 2-4 and Figure 2-5 present conductivity and relative permittivity values, respectively, for various tissues (liver, kidney, muscle, blood, breast fat) over the frequency range from $1 \mathrm{kHz}$ to $1 \mathrm{MHz}$, the $\beta$-dispersion region [16]. Tissue conductivities range in value from 0.01 to $1 \mathrm{~S} / \mathrm{m}$ while relative permittivities range from approximately $10^{2}$ to $10^{6}$. At very low frequencies the tissue relative permittivity is very large. The conductivity for each tissue 
increases with increasing frequency while the relative permittivity decreases with increasing frequency. It is apparent that the tissues are clearly differentiable by their conductivity values. The difference in tissue conductivity and relative permittivity values as well as their frequencydependent behavior provides a means for discriminating one tissue from another.

\section{Table 2-1: Permittivity Values}

Relative permittivity values of various biological $[16,17]$ and non-biological substances [22].

\begin{tabular}{|c|c|c|}
\hline Substance & $\begin{array}{c}\text { Relative } \\
\text { Permittivity } \\
\end{array}$ & Comments \\
\hline Water $\left(\mathrm{H}_{2} \mathrm{O}\right)$ & 78.54 & Temperature $=25^{\circ} \mathrm{C}, \mathrm{f} \cong 10^{8} \mathrm{~Hz}$, Liquid \\
\hline Benzene $\left(\mathrm{C}_{4} \mathrm{H}_{6}\right)$ & 2.274 & Temperature $=25^{\circ} \mathrm{C}, \mathrm{f} \cong 10^{8} \mathrm{~Hz}$, Liquid \\
\hline $\begin{array}{l}\text { Methanol } \\
\left(\mathrm{CH}_{4} \mathrm{O}\right)\end{array}$ & 32.63 & Temperature $=25^{\circ} \mathrm{C}, \mathrm{f} \cong 10^{8} \mathrm{~Hz}$, Liquid \\
\hline $\begin{array}{l}\text { Glycol } \\
\left(\mathrm{C}_{2} \mathrm{H}_{6} \mathrm{O}_{2}\right)\end{array}$ & 37.0 & Temperature $=25^{\circ} \mathrm{C}, \mathrm{f} \cong 10^{8} \mathrm{~Hz}$, Liquid \\
\hline $\begin{array}{l}\text { Glycerol } \\
\left(\mathrm{C}_{3} \mathrm{H}_{6} \mathrm{O}_{3}\right)\end{array}$ & 42.5 & Temperature $=25^{\circ} \mathrm{C}, \mathrm{f} \cong 10^{8} \mathrm{~Hz}$, Liquid \\
\hline $\begin{array}{l}\text { Calcium } \\
\text { Carbonate }\end{array}$ & 6.14 & $\begin{array}{l}\text { Temperature between } 17 \text { and } 22^{\circ} \mathrm{C}, \mathrm{f}=10^{6} \mathrm{~Hz} \text {, } \\
\text { Solid }\end{array}$ \\
\hline Ferrous Oxide & 14.2 & Temperature $15^{\circ} \mathrm{C}, \mathrm{f}=10^{8} \mathrm{~Hz}$, Solid \\
\hline $\begin{array}{l}\text { Potassium } \\
\text { Sulfate }\end{array}$ & 5.9 & $\begin{array}{l}\text { Temperature between } 17 \text { and } 22^{\circ} \mathrm{C}, \mathrm{f}=6^{*} 10^{7} \mathrm{~Hz} \text {, } \\
\text { Solid }\end{array}$ \\
\hline $\begin{array}{l}\text { Sodium } \\
\text { Chloride }\end{array}$ & 6.12 & $\begin{array}{l}\text { Temperature between } 17 \text { and } 22^{\circ} \mathrm{C}, \mathrm{f}=6^{*} 10^{7} \mathrm{~Hz} \text {, } \\
\text { Solid }\end{array}$ \\
\hline Lucite & 2.58 & Temperature $23^{\circ} \mathrm{C}, \mathrm{f}=10^{8} \mathrm{~Hz}$, Plastic \\
\hline Breast Fat & 543 & $\mathrm{f}=10 \mathrm{kHz}$ \\
\hline Liver & 28930 & $\mathrm{f}=10 \mathrm{kHz}$ \\
\hline Kidney & 38750 & $\mathrm{f}=10 \mathrm{kHz}$ \\
\hline Pancreas & 9190 & $\mathrm{f}=10 \mathrm{kHz}$ \\
\hline
\end{tabular}

\subsubsection{High Relative Permittivity Values for Biological Tissues}

Relative permittivity values for biological [16] and non-biological [22] medias are presented in

Table 2-1. These substances include both liquid and solid medias near room temperature (unless otherwise noted) and are quoted at $10 \mathrm{kHz}$ (except Lucite which is quoted at $10^{8} \mathrm{~Hz}$ ). Relative permittivity values for non-biological medias range from approximately 2.58 (Benzene) to approximately 80 (water). In contrast, the relative permittivity value for breast fat is nearly 550 at $10 \mathrm{kHz}$, with other tissues listed in Table 1 greater than this value by over an order of 
magnitude. It is evident that the relative permittivity values for biological media in the $\alpha$ - and $\beta$ dispersion regions are much greater than for non-biological medias.

\subsubsection{Differentiating Normal and Abnormal (cancerous) Tissue}

Numerous researchers have published their findings regarding electrical property differences between normal and abnormal neoplastic tissue [1,18, 19, 23, 24, 25]. Their results indicate that neoplastic cells are characterized by increased intracellular sodium, potassium, and other ionic content, resulting in higher intracellular conductivity than in normal cells. Neoplastic cells have a marked difference in the electrochemical properties of the cell membrane. Pethig reports cancerous cells possess greater relative permittivity than do normal tissues [19].

\subsubsection{Characteristics of Breast Cancer Lesions}

Surowiec, et al. [1] conducted a study of breast carcinoma and described three separate spatial classifications of tissue (Figure 2-6 and Figure 2-7): tumor bulk, infiltrating margins, and distant, normal tissue. Tumor bulk (the center of the tumor) is characterized by a high percentage of collagen, elastic fibers, and many tumor cells. The infiltrating margins are located between tumor bulk and normal tissue, and are characterized by few tumor cells and a large proportion of normally distributed collagen and fat from unaffected breast tissue. Finally, normal tissue is considered to be distant ( $2 \mathrm{~cm}$ or more) from the lesion. Other researchers use a similar classification for breast cancer [25, 26, 27, 28].

Breast cancer lesions are either in situ or infiltrating. In situ lesions remain confined within the epithelial tissue from which they originate. The lesion does not cross the basal membrane, thus the tumor and surrounding healthy tissue are of the same nature (epithelial). The abundance of malignant cells impacts the macroscopic conductivity (influenced by the increase in sodium and water) and relative permittivity (influenced by the differences in cell membrane electrochemistry) [18]. 


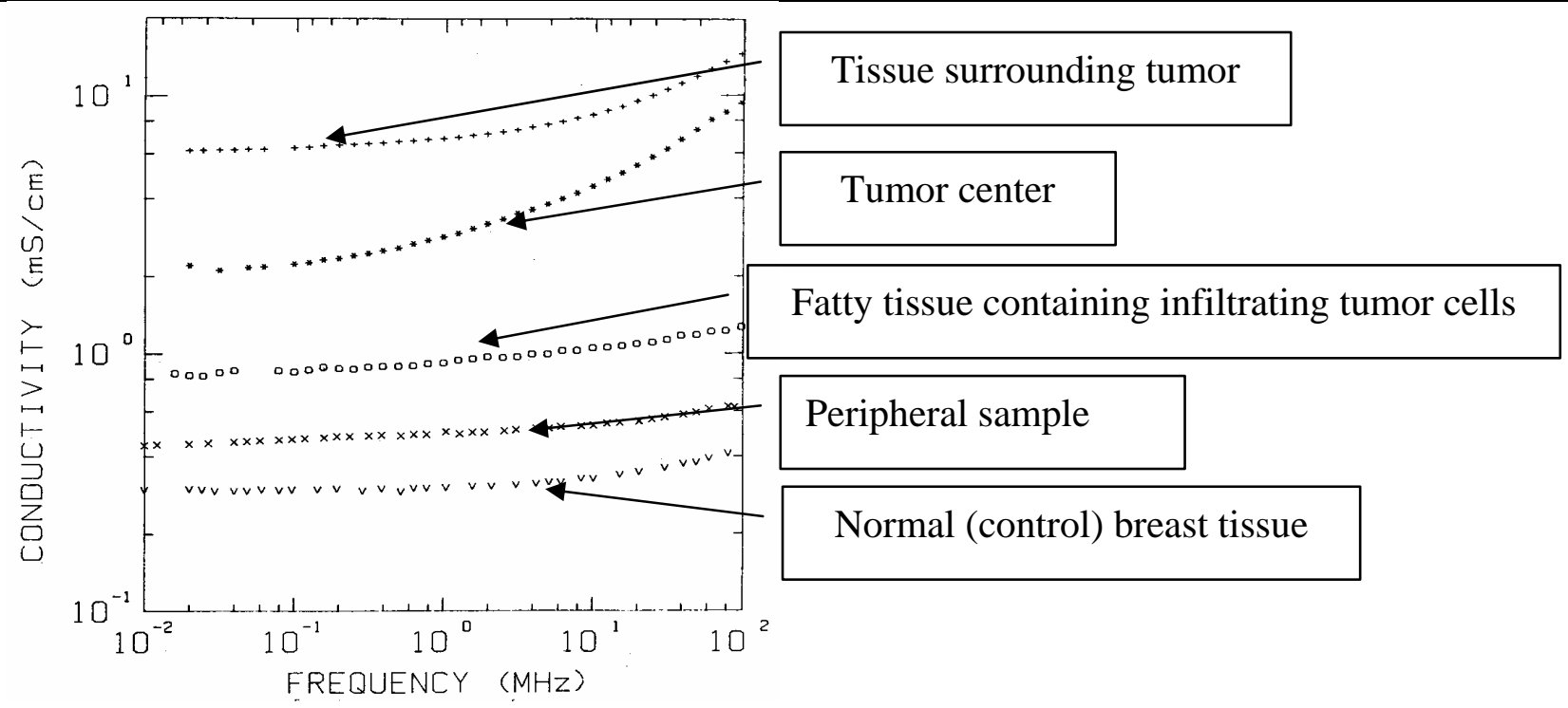

Figure 2-6: Frequency Dependence of Breast Cancer Conductivity

Conductivity of breast tumor measured at sites ranging from the tumor center to points distant from the center (healthy, normal tissue). (Surowiec, Figure 3 [1].)

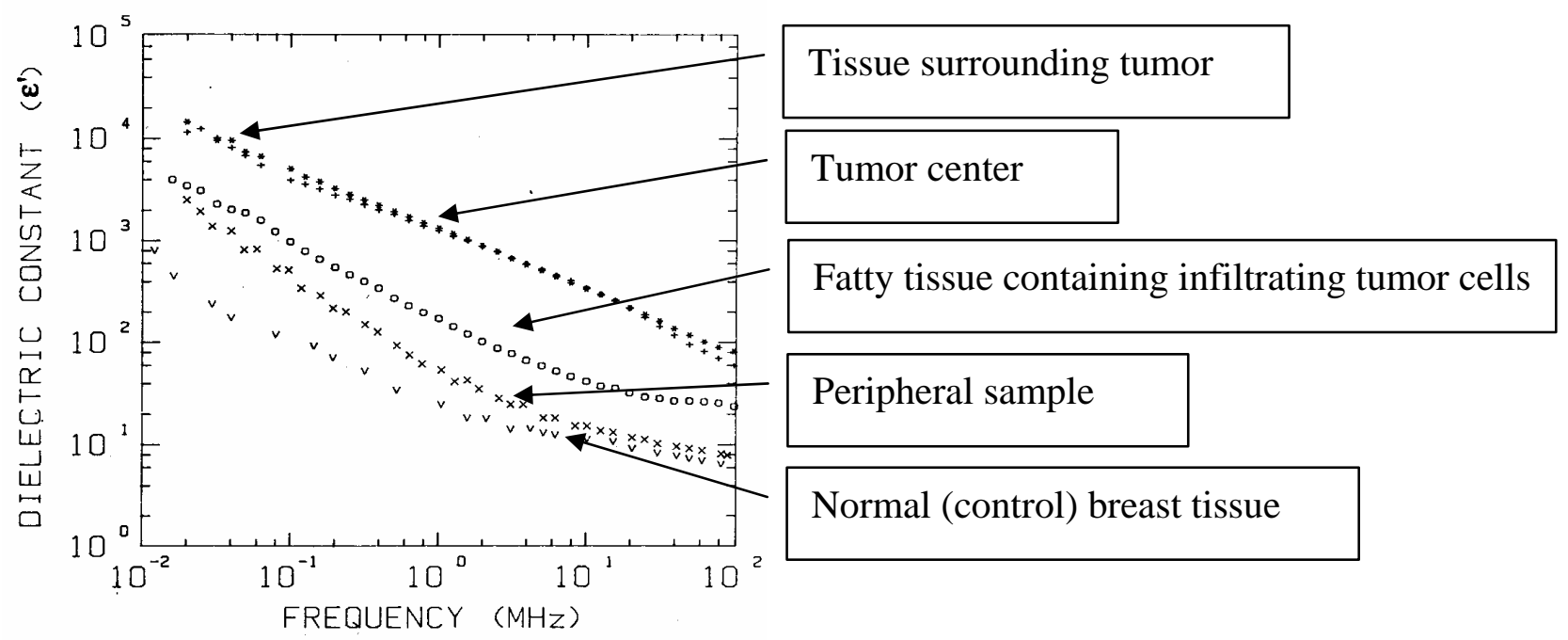

Figure 2-7: Frequency Dependence of Breast Cancer Relative permittivity

Relative permittivity of breast tumor measured at sites ranging from the tumor center to points distant from the center (healthy, normal tissue). (Surowiec, Figure 4 [1].)

Infiltrating lesions pass through the basal membrane. The malignant tissue has a different nature than surrounding normal tissue (epithelial vs. adipose). Epithelial tissue is compact and dense while adipose tissue is composed of large cells (having a high concentration of 
triglycerides). These structural differences affect the properties of tissue: first, normal tissue has a lower cellular density than does cancerous tissue. Secondly, cellular fluids within normal tissue are not as abundant as in epithelial cells . Generally the radii of epithelial cells are smaller than in adipose cells, which implies that the radii of cancerous cells will also be less than for healthy cells. The fractional volume of cancerous cells is greater than in normal tissue because the epithelial population is relatively greater than in normal breast tissue composed largely of adipose cells. Lastly, the intracellular conductivity of cancerous cells is greater than for normal cells, and the extracellular conductivity is also higher because of the abundance of extracellular fluid - resulting from larger gaps between normal and cancerous cells). Thus, the conductivity of the infiltrated tissue is greater than for normal, healthy breast tissue [18, 23, 24, 25].

\subsubsection{Temperature Dependence of Tissue Conductivity and Relative Permittivity}

Tissue impedance depends on tissue temperature. Webster [21] reports that impedance decreases with increasing temperature, but the effect depends on applied frequency and water content of the tissue. Pethig [29] reports relative resistivity $(\Delta \rho / \rho)$ changes of approximately $0.01 /{ }^{\circ} \mathrm{C}$ at $1 \mathrm{MHz}$. Zheng [30] reports that muscle impedance varies by $-2 \% /{ }^{\circ} \mathrm{C}$ between $5^{\circ} \mathrm{C}$ and $40^{\circ} \mathrm{C}$. Schwan [12] catalogued resistivity and relative permittivity temperature dependence in the 50-900 MHz frequency range. The temperature dependence is a property that has enabled monitoring tissue temperature during hyperthermia treatment by monitoring changes in tissue resistivity [31].

\subsubsection{Anisotropic Dependence on Tissue Conductivity and Relative Permittivity}

The bioelectric properties of muscles fibers in the body are anisotropic, i.e., the conductivity and relative permittivity of the muscle tissue at a given location varies in magnitude depending on the direction of the applied electric field [30, 32, 33, 34]. 


\subsection{Medical Applications Using Biological Electrical Properties}

The electrical characteristics of biological tissues have been exploited for a variety of medical applications, including breast cancer screening [23, 35, 36, 37, 38, 39, 40,41, 42] lymph node screening [43, 44, 45, 46] and in instrumentation used for gastrointestinal [47, 48, 49, 50], pulmonary [51, 52, 53, 54, 55, 56, 57, 58, 59, 60, 61, 62], neurological [63, 64, 65, 66, 67], and cryosurgical applications [68, 69, 70]. Breast cancer screening is a particularly interesting and relevant medical application. Breast cancers are the most commonly diagnosed neoplasm in women (excluding nonmelanoma skin cancer) and are the second leading cause of cancer death today. In the United States in 2004 approximately 216,000 invasive and 59,000 in situ breast cancer cases were diagnosed resulting in an estimated mortality of 40,000 women for this disease. Medical experts have determined that earlier detection can lead to a decline in breast cancer mortality [71]. Because of its high incidence and prevalence in the female population, breast cancer screening will receive special attention in this survey.

\subsubsection{Breast Cancer Screening}

Three different recently developed impedance imaging techniques for breast cancer screening have been developed and clinically tested. These systems are the TSCAN 2000, a commercially available system distributed by Siemens Medical Systems, CENTILLION, a prePMA (pre market approval) system marketed by TCI Medical, and an Electrical Impedance Spectroscopy (EIS) system developed by the Breast Cancer Screen Project at Dartmouth College.

The TSCAN 2000 [35] is a two-component device; one component is a handheld scanning instrument applied directly to the patient's breast while the second component is a metal rod held in the patient's hand. The two components are maintained at different electric potentials resulting in a small current flow through the breast. This current is sensed and measured by the planar array of sensors mounted in the handheld instrument and the acquired electrical data is used to render two-dimensional conductivity images of the breast.

The TSCAN 2000 has shown some promise as a scanning device to augment standard mammograms. Initial published results showed that $93.1 \%$ of malignant lesions and $65.5 \%$ of benign lesions were correctly identified [36]. A later study on 210 women collectively having 
240 suspicious findings on sonogram or mammogram correctly identified 86 of 103 malignant and 91 of 137 benign lesions (87.8\% sensitivity, 66.4\% specificity) [37]. A separate study, conducted on 208 women collectively having with 150 masses and 114 micro-calcifications, investigated the influence of lesion size on the detection rate and concluded that the highest detection rates were observed for 10-30-mm tumor masses and for invasive ductal carcinomas and noninvasive cancers [38].

CENTILLION is likewise a two component system similar to the TSCAN 2000. The device is approximately the same size as the TSCAN 2000 but possesses a far greater number (256 vs. 16) of electrodes for sensing the breast's electrical properties. The system injects a current through a single electrode and measures electric potential on the remaining electrodes. A significant difference between Centillion and TSCAN 2000 is that Centillion generates threedimensional images of breast tissues [39] whereas TSCAN 2000 generates only 2D images. Initial clinical results showed that regions identified as having increased conductivity are typically associated with increased vascularity that is associated with tumor growth. Such focal abnormalities are found in $67 \%$ of patients with breast cancer. CENTILLION is sold by TCInternational, Inc, which is continuing its research. [40].

The EIS breast scanner at Dartmouth College differs significantly from the previous two systems. First, the system is not handheld, but rather embedded in an examination table upon which the patient lays prone with breast hanging pendant through an opening in the table. A ring of 32 pneumatically actuated electrodes is pressed against the patient's breast. The system injects current, measures potentials and generates two-dimensional images of the complex impedance distribution. Spatial distributions of conductivity and relative permittivity of the tissue are subsequently reconstructed from the complex impedance values acquired by the measurement system. Initial results on 26 subjects identified $83 \%$ of the American College of Radiology Breast Imaging Reporting and Database System category (ACR BI-RADS) 4-5 lesions using a visual criterion while identifying just $67 \%$ using a numerical criterion [23, 41]. A later study suggests that Electrical Impedance Spectroscopy breast exams were capable of locating tumors with better than $1 \mathrm{~cm}$ accuracy, provided the electrode placement is well controlled [42]. 


\subsection{The Electrical Impedance Tomography}

Electrical Impedance Tomography (EIT) is an imaging modality that renders images or 'representations' of the distribution of electrical properties within a medium. This section introduces and defines mathematical notation, introduces the physical problem and assumptions commonly used to solve the EIT problem, and, finally, introduces and discusses different boundary conditions. Methods for solving the EIT problem and reconstructing images are discussed in the next section. This reconstruction is computed from currents that are measured after an ac voltage is applied through individual electrodes. The distribution of complex electrical properties (conductivity and relative permittivity) is computed and rendered from measured currents, voltages and electrode position data.

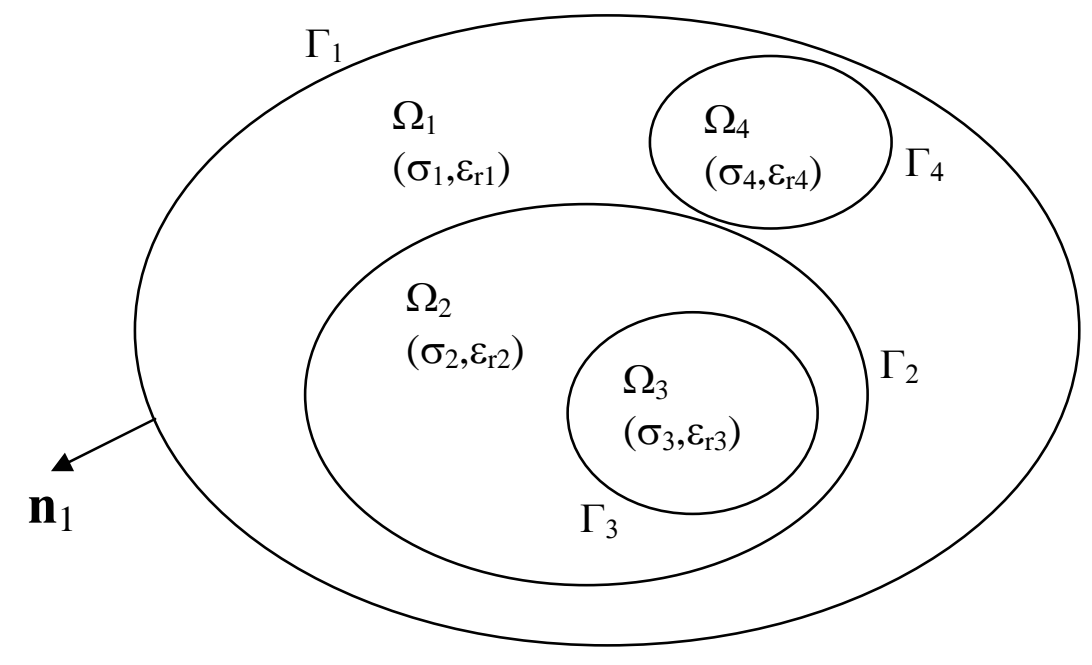

Figure 2-8: Mathematical domain and sub-domains

A mathematical representation of region to be imaged, divided into domains $\left(\Omega_{1}, \Omega_{2}\right.$, and $\left.\Omega_{3}\right)$ and boundaries $\left(\Gamma_{1}, \Gamma_{2}\right.$, and $\left.\Gamma_{3}\right)$. Within each domain, conductivity and permittivity $\left(\sigma\right.$ and $\left.\varepsilon_{\mathrm{r}}\right)$ are uniform and constant.

Notation introduced in this section is based on the work of Morucci and Marsili [72]. A mathematical domain $\Omega_{1}$ having a boundary $\Gamma_{1}$ is illustrated in Figure 2-8. It is open, bounded, and "regular" with $\mathrm{C}^{\mathrm{n}}$ in general $\mathrm{n}=2$ or 3 ( $\mathrm{C}$ for complex domain). Within the domain is a distribution of electrical properties (conductivity $\sigma$ and relative permittivity $\varepsilon_{\mathrm{r}}$ ) represented as a complex conductivity, $\alpha^{*}=\alpha+j \omega \varepsilon_{0} \varepsilon_{\mathrm{r}}$. The distribution is modeled as a collection of sub-domains, with each sub-domain possessing a piecewise, constant complex conductivity. Four domains and 
boundaries, $\left[\left(\Omega_{1}, \Gamma_{1}\right),\left(\Omega_{2}, \Gamma_{2}\right),\left(\Omega_{3}, \Gamma_{3}\right)\right.$, and $\left.\left(\Omega_{4}, \Gamma_{4}\right)\right]$, are illustrated. The unit vector of the normal to $\Gamma_{1}, \underline{\hat{\mathbf{n}}}$, is oriented outward from $\Omega_{1}$. Finally, boundary conditions and external measurements are imposed at the boundary surface of $\Gamma_{1}$.

Problems involving time-varying electric and magnetic fields are described by Maxwell's equations ((2-1) to (2-4)) [74], where $\nabla$ is the divergence operator, $\mathbf{E}$ is the electric field intensity, $\mathbf{D}$ is the electric flux density, $\mathbf{H}$ is the magnetic field intensity and $\mathbf{B}$ the magnetic flux density.

$$
\begin{gathered}
\nabla \cdot \mathbf{D}=\rho \\
\nabla \cdot \mathbf{B}=0 \\
\nabla \times \mathbf{E}=-\frac{\partial \mathbf{B}}{\partial t} \\
\nabla \times \mathbf{H}=\mathbf{J}+\frac{\partial \mathbf{D}}{\partial t}
\end{gathered}
$$

Maxwell's equations are used to derive a relationship between the complex conductivity distribution, $\sigma^{*}$, and the potential field, $\Phi$. The result is Poisson's equation, (2-5), which is an elliptic partial differential equation. The derivation assumes negligible magnetic phenomena $(\mu=0$ ), an isotropic medium (which may be an imperfect assumption), charge conservation, and preponderant electrostatic phenomenon [73] (i.e., the time derivative of the B field is ignored in the Maxwell equations above).

$$
\begin{gathered}
\nabla \cdot\left(\sigma^{*} \nabla \Phi\right)=0 \text { in } \Omega \\
+ \text { boundary conditions on } \Gamma_{1}
\end{gathered}
$$

Determining $\sigma^{*}$ in $\Omega$ is achieved by solving equation (2-5) subject to the imposed boundary conditions. Dirichlet, Neumann, mixed, or Cauchy problem boundary conditions are summarized in Table 2-2; the specific boundary conditions dictate the type of problem to be solved. Typically either the Dirichlet (currents are measured resulting from an applied potential) or Neumann (potential is measured resulting from an injected current) boundary conditions are employed. 
Table 2-2: Boundary Conditions Matrix for the Direct Problem

(adapted from Table 1 in Morucci and Marsili [72])

\begin{tabular}{|c|c|c|c|c|}
\hline & $\begin{array}{l}\text { Dirichlet type } \\
\text { problem }^{(a)}\end{array}$ & $\begin{array}{l}\text { Neuman type } \\
\text { problem }^{(b)}\end{array}$ & $\begin{array}{l}\text { Mixed type } \\
\text { problem }^{(\mathrm{c})}\end{array}$ & $\begin{array}{l}\text { Cauchy type } \\
\text { problem }^{(\mathrm{d})}\end{array}$ \\
\hline Formulation & $\begin{array}{c}\nabla\left(\sigma^{*} \nabla \Phi\right)=0 \\
\text { in } \Omega \\
\Phi_{\Gamma}=\mathrm{g} \\
\\
\text { g (voltage } \\
\text { values) given } \\
\text { on } \Gamma\end{array}$ & $\begin{array}{c}\nabla\left(\sigma^{*} \nabla \Phi\right)=0 \\
\text { in } \Omega \\
\sigma^{*} \frac{\partial \Phi}{\partial \overrightarrow{\mathbf{n}}}=0 \\
\text { on } \Gamma \backslash \Gamma_{\mathrm{a}} \\
\sigma^{*} \frac{\partial \Phi}{\partial \overrightarrow{\mathbf{n}}}=\mathrm{j}\end{array}$ & $\begin{array}{c}\nabla\left(\sigma^{*} \nabla \Phi\right)=0 \\
\text { in } \Omega \\
\sigma^{*} \frac{\partial \Phi}{\partial \overrightarrow{\mathbf{n}}}=\mathrm{j} \\
\text { on } \Gamma_{\mathrm{a}} \\
\Phi_{\mathrm{i}}=\Phi_{\mathrm{m}} \\
\text { on } \Gamma \backslash \Gamma_{\mathrm{a}}\end{array}$ & $\begin{array}{c}\nabla\left(\sigma^{*} \nabla \Phi\right)=0 \\
\text { in } \Omega \\
\sigma^{*} \frac{\partial \Phi}{\partial \overrightarrow{\mathbf{n}}}=0 \\
\text { on } \Gamma \backslash \Gamma_{\mathrm{a}} \\
\Phi=\Phi_{\mathrm{m}} \\
\text { on } \Gamma \backslash \Gamma_{\mathrm{a}}\end{array}$ \\
\hline Characteristic & $\begin{array}{l}\text { Voltage } \\
\text { problem on } \Gamma\end{array}$ & $\begin{array}{l}\text { Current flow } \\
\text { problem }\end{array}$ & $\begin{array}{l}\text { Piecewise } \\
\text { voltage or } \\
\text { current flow } \\
\text { problem }\end{array}$ & $\begin{array}{l}\text { Simultaneous } \\
\text { voltage and } \\
\text { current flow } \\
\text { problem }\end{array}$ \\
\hline
\end{tabular}

Notes:

a) Voltage at the boundary $\Gamma$ is known, current is measured

b) Current at the boundary $\Gamma$ is known, voltage is measured

c) Voltage and current are known at different points of the boundary $\Gamma$

d) Voltage and current are known at the same points of the boundary $\Gamma$. Cauchy problem is characterized by the presence of redundant conditions on one part $\left(\Gamma \backslash \Gamma_{\mathrm{a}}=\Gamma \cap \sigma \Gamma_{\mathrm{a}}\right)$

\subsection{Image Reconstruction Methods in EIT}

Image reconstruction consists of solving equation (2-5) using either analytical or numerical methods. The analytical method solves equation (2-5) using a set of known, welldefined functions (sine, cosine, Bessel, etc.), resulting in continuous, or piecewise continuous solutions throughout the domain. Jackson [74] solves some simple cases analytically, however, the analytical method is better suited to simple cases where internal structures are known $a$ priori, which is rarely the case. Consequently, EIT images are more typically generated using numerical methods.

Numerical methods compute the electrical property distribution using numerical values at the boundary of the medium, the measured output signal and electrode position information. The results are expressed as discrete numerical values, and not continuous or piece-wise continuous functions. Due to the computational complexity and high processing times required, results are 
generally (but not always) piecewise constant or solutions for discrete points. Numerical methods are generally grouped as either the direct or indirect (iterative) method.

\subsubsection{Early EIT Research}

Henderson and Webster report the earliest attempt to image the internal resistance of the human body in 1978 [75]. They developed an impedance camera that conducted spatially specific measurements of the thorax. Although the images produced by this camera were not strictly tomographic, (i.e. the representation was not for a single plane), the method is noteworthy because it produced a representation of the impedance distribution in the chest. In 1983, Barber and Brown [72] reported the first true electrical impedance tomograph that produced tomographic images, using a system that applied a potential to the subject, measured the resulting currents and rendered images using the back projection method. Additional results were reported the following year [76].

In subsequent years several other methods were introduced. Murai and Kagawa [77] developed the sensitivity reconstruction method based on Geselowitz's sensitivity theorem [78]. Kim, et al. [79], proposed a perturbation method for image reconstruction derived from Gilbert's [80] Simultaneous Iterative Reconstruction Technique (SIRT). Yorkey, et al. [81] later improved on this method. Wexler, et al. [82] introduced the double constraint method which uses finite element methods [83] to derive internal resistivity distributions by first computing the internal current density with known current sources followed by computation of potential gradients at internal elements using measured voltage and current source data.

Yorkey $[2,81,84]$ introduced the modified Newton-Raphson (N-R) method. This method, which has been cited nearly 200 times, persists as one of the most common and accurate methods. This method typically uses finite element methods [83] to compute a forward solution, followed by a Newton-Raphson computation of the inverse solution of the impedance problem. Yorkey modified the basic Newton-Raphson method by including a regularization term in the update equation; this addresses the underdetermined nature of the EIT problem when finite element methods are employed. An underdetermined problem results in ill-posedness, yielding a solution that may be quite susceptible to small errors due to measurement noise. Yorkey, et al. [84] compared the sensitivity reconstruction method, the perturbation method, the Simultaneous Iterative Reconstruction Technique, Wexler's double constraint method, and the modified 
Newton-Raphson method. Their conclusion was that the Newton-Raphson method yielded the best overall results that converge in all instances. The disadvantage of the N-R method is that it is computationally intensive, thus requiring large amounts of system memory, disk space, and computational time. The Newton-Raphson method will be described in greater detail in subsequent sections of this chapter.

\subsubsection{Reviews of EIT}

In 1989, Barber [85] published a review of image-reconstruction techniques for electrical-impedance tomography. In 1990, Webster [21] edited a textbook covering the basic theory of the impedance properties of biological tissues, data acquisition systems, reconstruction algorithms, and performance criteria in electrical impedance tomography. In 1996, Morucci and Marsili [72] compiled a highly comprehensive and instructional review that covers all work from the early discoveries by Barber and Brown to the mid 1990’s. In 1997, The European Concerted Action on Impedance Tomography published a report on imaging with electricity [86]. Finally, in 1999 Cheney, et al. [87] published a survey of research performed at RPI in electrical impedance tomography.

\subsubsection{Direct Method}

The direct method uses the boundary condition values, the measured signal and electrode positional information to 'directly' compute the electrical property distribution without intermediate computations. Generally, direct method solutions attempt to convert the non-linear Poisson's equation into a linear equation, although the non-linear nature of the problem is difficult to avoid.

Over the years a number of direct methods have been proposed. Among the most notable are the back projection method [88, 89, 90, 91, 92], the sensitivity method [77, 93, 94], the layer stripping method [95], the Newton One Step Error Reconstructor method [96, 97, 98 99], and Nachman’s Dirichlet to Neuman mapping method [100, 101, 102].

\subsubsection{Indirect Method}

The indirect method, illustrated in Figure 2-9, solves Poisson's equation by computing intermediate results. The process is divided into two parts, the forward solution and the inverse 
solution. The forward solution combines an electrical property estimate with boundary conditions to produce a prediction of the measured output signal (either the current or voltage). An error term is computed from the predicted and measured output signals. The inverse solution then uses the error term to compute an adjustment to the electrical property estimate that minimizes the magnitude of the error term. The process of predicting signal and adjusting electrical properties is repeated until an arbitrary convergence criterion is achieved.

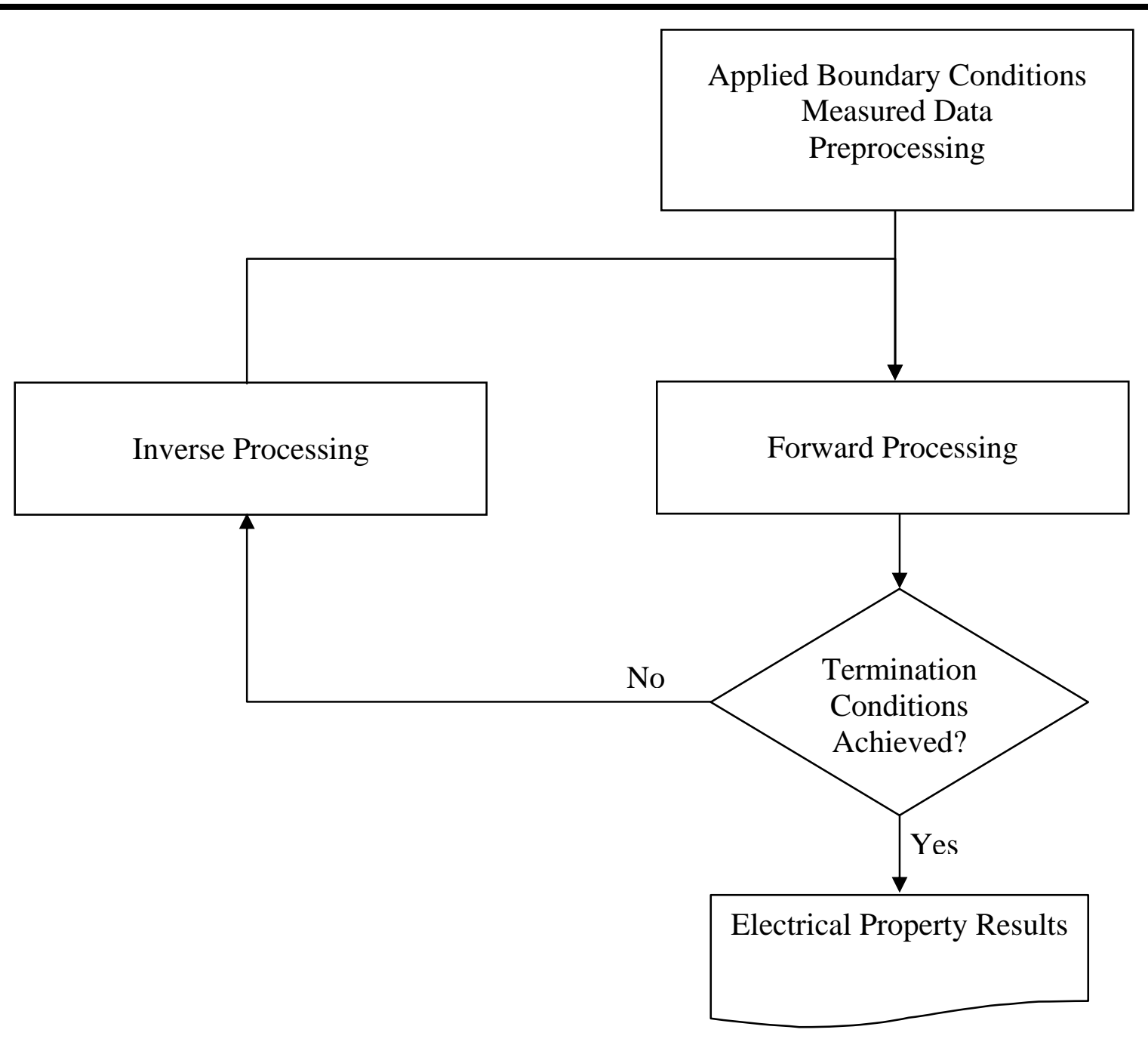

Figure 2-9: Functional Flow Chart of the Indirect Reconstruction Method

The flow chart illustrates the indirect reconstruction method. Following initial preprocessing, the voltage along the boundary of the domain is computed by the forward process and the predicted electrical properties are updated by the inverse process. Both forward and inverse process are repeated until termination conditions are achieved. 


\subsubsection{Forward Solution}

The forward problem uses numerical methods that combine boundary condition values and estimates of the electrical property distribution to compute predictions of the output signal. For Dirichlet boundary conditions, a potential is applied and current serves as the output signal. The domain, a continuous region, is modeled using a discretized representation of piecewise constant sub-domains, a process that maps numerical values to specific points and regions in the domain. The problem is transformed into a system of linear equations, which are solved numerically.

\subsubsection{Finite Difference, Finite Element, and Boundary Element Methods}

Finite element methods (FEM), finite difference methods (FDM), and boundary element methods (BEM) are three numerical methods used to perform the forward solution. FDM and FEM require discretization of the entire domain under study. These methods produce a piecewise approximation to the differential equation form of the Poisson equation. The BEM gives a point wise approximation and solves the integral formulation of the Poisson equation. BEM is better suited to arbitrarily shaped boundaries. All three of these methods are described in greater detail in [103, 104, and 105].

The finite difference method has been implemented by a number of authors. Gregory and Gregory [4] used the FDM in simulation studies to investigate the effectiveness of the Electrical Property Enhanced Tomography (EPET) method. Their studies considered four regions of the human body (Brain/head, thorax, pelvis, and thigh), and finite difference maps were developed from CT and MR data supplied by the Visible Human Project [106]. Ider, et al. [107], performed 2D simulations in which they applied an FDM-based algorithm in image reconstruction for magnetic resonance-electrical impedance tomography (MR-EIT). Zhao, et al. [108, 109], used an FDM approach to estimate multi-layer tissue conductivities using divided electrodes. Finally, Patterson and Zhang [110] used a finite difference human thorax model as a phantom to evaluate an EIT reconstruction algorithm.

Finite element methods have been cited nearly 200 times. Paulsen and Jiang [31] developed a finite element method to track subsurface temperature distributions during hyperthermia. Their model minimizes the total variation in a dual-mesh FEM implementation with spatial low-pass filtering. Glidewell and $\mathrm{Ng}$ [142, 141] implemented 2D and 3D finite element models and methods and applied these to the region of a canine thorax. Their research was concerned with the anisotropic properties of cardiac tissue. Bayford, et al. [111], developed 
finite element models and methods for the head. Borsic, et al. [112], developed a 2D finite element model and method for the human thorax. And finally, Osterman, et al. [23], and Kerner, et al. [41 and 42], applied a 2D finite element model and method in the context of breast cancer screening.

The boundary element method has found some application in EIT. Marsili, et al. [113], considered a dipole representation in which the optimized position, orientation and length are used to model discontinuities in conductivity. Shi, et al. [114], studied conductivity changes and boundary potential differences, and evaluated the sensitivity coefficients. De Munck, et al. [115, 116, 117], introduced a parametric formulation that treats position, orientation, size, and conductivity as unknowns and solves the forward problem by adjusting parameters to optimize the fit to EIT data. Other researchers using BEM are Jain, et al. [118], in 1997, Duraiswami, et al. [119], in 1998, and Tarvainen, et al. [120], in 2001.

\subsubsection{Inverse Solution}

The inverse problem, as illustrated in Figure 2-9, updates the electrical property estimates used in the forward problem by minimizing an error term. The indirect reconstruction method concept presumes that the error term approaches a minimum as the estimate of the electrical property distribution approaches the actual distribution. Based on the number of publications, the Newton-Raphson (N-R) method is by far the most commonly used inverse method in EIT. There are a number of other methods aside from N-R. These include the Sensitivity Method [121, 122, 123, 124, 125, 126], Neural Networks [127, 128, 129, 130], and Wexler's double constraint method [82]. This review will focus on the Newton-Raphson method.

\subsubsection{The Modified Newton-Raphson Method}

The Newton-Raphson method for Neumann boundary conditions is defined by equations (2-6) and (2-7), where $\Phi_{m}$ is the measured potential at the electrodes, $\Phi(\sigma)$ is the predicted potential at the electrodes and $\sigma$ is the estimated conductivity distribution within the domain $\Omega$.

$$
\begin{gathered}
\text { error }=\sum_{\text {all independent measurements }}\left(\Phi(\sigma)-\Phi_{m}\right)^{2} \\
\min _{\sigma}\{\text { error }\} \Rightarrow \frac{\partial e r r o r}{\partial \sigma}=0
\end{gathered}
$$

The error term is minimized and the resultant equation is solved for $\sigma$. This process requires performing a matrix inversion on $\left[\Phi^{\prime}(\sigma)\right]^{T}\left[\Phi^{\prime}(\sigma)\right]$. The problem is ill-posed, leading to 
a poorly conditioned matrix and an unstable matrix inversion. Consequently, regularization techniques such as the Tikhonov regularization scheme (equation (2-8)), are employed. Tikhonov regularization includes a regularization matrix, $\mathrm{RM}(\sigma)$, and regularization parameter, $\mu$, together with the error term. Equations (2-9) and (2-10) present an update scheme derived by Yorkey [84] that includes a Marquardt regularization term, where the index k reflects the number of iterations for which the conductivity has been updated.

$$
\begin{gathered}
\min _{\rho}\left\{\text { error }+\mu\|R M(\sigma)\|^{2}\right\} \\
\sigma_{k+1}=\sigma_{k}-\left\{\left[\Phi^{\prime}\left(\sigma_{k}\right)\right]^{T}\left[\Phi^{\prime}\left(\sigma_{k}\right)\right]+\mu \mathbf{I}\right\}^{-1}\left[\Phi^{\prime}\left(\sigma_{k}\right)\right]^{T}\left[\Phi\left(\sigma_{k}\right)-\Phi_{m}\right] \\
\Phi^{\prime}(\sigma)=\frac{\partial \Phi(\sigma)}{\partial \sigma}
\end{gathered}
$$

Yorkey, et al. [84], concluded that the N-R method always converges iteratively to a solution that yields the smallest residual error when compared with other methods. The N-R method has been modified, improved, and extended over the years. The method was shown to be unique [131], although unstable [132] —in fact, since the method can suffer from severe illposedness, no method has been developed to improve stability through a closed-form mathematical approach [133]

Woo, et al. [134], implemented Hachtel's augmented matrix method to develop an efficient and robust image reconstruction algorithm for static impedance imaging. This implementation reduces the undesirable effects of the ill-conditioned Hessian matrix. To overcome high computation time, the algorithm was implemented on a parallel computer system, reducing computation time from hours to minutes. Artola and Dell [135] implement a Broyden quasi-Newton approach on a simulated 30 by 30 discretized region. The simulation solves the forward problem and updates the inverse of the Jacobian matrix in an average of $0.11 \mathrm{~s}$ on a 25 $\mathrm{MHz}$ NeXT station using the 68040 processor. An average of 20 iterations is required for accurate image reconstruction. Loh and Dickin [136] implemented a sparse matrix method to avoid redundant computations. These improvements reduce overall computational complexity in the original modified Newton Raphson algorithm by a factor of 5 .

Grootveld, et al. [137], present a regularized, modified Newton-Raphson algorithm that limits the number of elements used by the algorithm to the number of true non-zero eigenvalues. 
This approach reduces ill-conditioning effects by implementing a mapping scheme from a userdefined pixel mesh to a standard finite-element mesh. Edic, et al. [138], implemented an N-R method that produces images of admittivity or conductivity, and relative permittivity. Rao, et al. [139], implemented a method based on the notion of homotopy. This method continuously maps solution space. After a few initial mapping solutions, the solution behavior is restrained towards the global convergence. Lastly, Player, et al. [140], implemented a truncated a 3-dimensional Newton algorithm that avoids computational delays through the use of a preconditioned conjugate gradient (PCG) solution of the Levenberg-Marquardt update.

\subsubsection{Using a priori anatomical information}

Aside from regularization, another approach for improving the N-R method is through the use of a priori anatomical information. The information is acquired through CAT or MRI images and is used in the creation of the FE or FD meshes. Furthermore, the assignment and update of estimated electrical property values could be constrained based on tissue group; mesh elements corresponding to a muscle tissue region can be constrained to possess similar values. Groups investigating the use of anatomical information are Gregory and Gregory [4], Glidewell and Ng [141, 142], Baysal and Eyuboglu[143, 144, 145], and the Vauhkonen and Kaipio group $[146,147]$.

Glidewell and Ng [141] introduced the use of anatomical constraints to address anisotropy in static EIT, using a two-step approach. First, the boundaries between regions of different conductivities were constrained anatomically using magnetic resonance imaging (MRI) data. Second, the conductivity values in different regions were determined, including regions of anisotropic conductivity. This approach was intended to better model muscle regions (e.g., skeletal muscle) that possess greater conductivity in the direction parallel to the muscle fiber. Simulation studies conducted on a canine torso, demonstrated that anisotropic effects couldn't be ignored. Glidewell and Ng [142] extended the method to a realistic three-dimensional (3-D) case.

Baysal and Eyuboglu [143] used a priori geometrical information to compare minimum mean squares error estimator (MiMSEE) with least squares error estimator (LSEE). The LSEE is the error estimator method commonly used in the modified N-R method. This group hypothesized that statistical properties of regional resistivities, linearization error, and instrumentation noise needed to be incorporated into a new resistivity estimation algorithm. 
MiMSEE uses geometrical information from a separate high-resolution imaging modality (CAT or MRI). The MiMSEE method was examined in simulation studies and reduced errors by a factor of 27 over the LSEE, but required more than 20 times more computational time than the LSEE. Baysal and Eyuboglu [144] applied the method to experimental data obtained from two different phantom geometries. They concluded that the MiMSEE is more robust than the LSEE. Finally, Baysal and Eyuboglu [145] examined geometrical uncertainties due to organ boundary variation and electrode position uncertainties. They showed that the MiMSEE yields an estimation error ranging between 17 and 64 times lower than for the LSEE. They concluded that the MiMSEE is robust even with geometrical uncertainties.

Vauhkonen, et al. [146], implemented prior information in the form of approximate images. In their technique, an eigen-image method using principal component analysis is generated from a number of similar conductivities based on the approximation of the prior covariance matrix by simulated samples of feasible conductivities. The eigenfunctions form a basis set of functions that are used to create the mesh for FE analysis. Kaipio, et al. [147], report a method based on the generalized Tikhonov regularization and on generally approximate spatial information. A regularization operator incorporates the $a$ priori information with a properly constructed matrix-valued field. 


\section{THEORY}

The previous chapter introduced and described Electrical Impedance Tomography (EIT), a technique for determining conductivity and relative permittivity distributions within the human body. EIT relies mainly on numerical methods to solve the non-linear Poisson's equation (equation (3-1)) [18, 21, 74]. Both direct (single iteration) and indirect (multiple iteration) methods are used, but indirect methods are the dominant method of choice.

$$
\nabla\left(\left(\sigma+j \omega \varepsilon_{0} \varepsilon_{r}\right) \nabla \Phi\right)=0
$$

where: $\Phi$ is the potential field in volts

$\sigma$ is the conductivity in $\mathrm{S} / \mathrm{m}$

$\varepsilon_{\mathrm{r}}$ is the relative relative permittivity

$\omega$ is the radial frequency in rads/s

$\varepsilon_{0}$ is the relative permittivity of free space in Coulombs/Volt/m

The indirect method is divided into a forward and an inverse problem. Typically the forward problem uses either Finite Element or Finite Difference Methods (FEM or FDM) to compute predictions of the measured boundary values using estimates of the internal conductivity and relative permittivity values. The inverse problem uses the Newton-Raphson (N-R) method to update these estimated electrical properties by minimizing the least square error estimate (LSEE) between the predicted and measured boundary values. The forward and inverse problems are performed sequentially and iteratively until the LSEE falls below a minimum threshold. The N-R method produces a matrix representation that is ill-posed. The matrix must be inverted, which produces erroneous results. The ill-posed issues are generally addressed using regularization or a priori geometric information.

This research proposes an alternative approach to solving the Poisson equation. This approach solves the Green's second identity (equation (3-2)), also referred to as Green’s theorem, using Dirichlet boundary conditions. Green's theorem uses a function, $\mathrm{G}_{\mathrm{D}}\left(\mathbf{r}, \mathbf{r}^{\prime}\right)$, to relate internal charge distribution, $\rho\left(\mathbf{r}^{\prime}\right)$, and applied boundary potential, $\Phi_{\mathrm{S}}$, to the internal potential field $\Phi(\mathbf{r})$. The function $\mathrm{G}_{\mathrm{D}}$, the Green's Function (or geometric function), is determined solely by the shape of the domain. The internal potential field is a function only of the shape of the domain, the boundary conditions and the location and magnitude of the internal charge distribution, as follows: 


$$
\Phi(\mathbf{r})=\frac{1}{4 \pi \varepsilon_{0}}\left[\int_{V} \rho\left(\mathbf{r}^{\prime}\right) G_{D}\left(\mathbf{r}, \mathbf{r}^{\prime}\right) d^{3} x^{\prime}-\varepsilon_{0} \oint_{S} \Phi_{S}\left(\mathbf{r}^{\prime}\right) \frac{\partial G_{D}}{\partial n^{\prime}} d a^{\prime}\right]
$$

An expression relating the internal charge distribution to the electrical properties, $\sigma$ and $\varepsilon_{\mathrm{r}}$, is derived from equation (3-1). The left most gradient in equation (3-1) is applied to both the electrical property term and the potential term resulting in equation (3-3). The charge density, $\rho$, is related to the $\nabla^{2} \Phi$ term (equation (3-4)), one of Maxwell's equations [74]. Equation (3-4) is substituted into equation (3-3), which is then rearranged to solve for the charge density, $\rho$ (equation (3-5)).

$$
\begin{aligned}
\nabla\left(\sigma+j \omega \varepsilon_{0} \varepsilon_{r}\right) \cdot \nabla \Phi & =-\left(\sigma+j \omega \varepsilon_{0} \varepsilon_{r}\right) \nabla^{2} \Phi \\
\nabla^{2} \Phi & =-\frac{\rho}{\varepsilon_{0}}
\end{aligned}
$$

where: $\rho$ is the charge density in Coulombs $/ \mathrm{m}^{3}$

$$
\rho\left(\mathbf{r}^{\prime}\right)=\frac{q\left(\mathbf{r}^{\prime}\right)}{\text { volume }}=\varepsilon_{0} \frac{\nabla\left(\sigma+j \omega \varepsilon_{0} \varepsilon_{r}\right)}{\left(\sigma+j \omega \varepsilon_{0} \varepsilon_{r}\right)} \cdot \nabla \Phi\left(\mathbf{r}^{\prime}\right)
$$

The charge density, $\rho$, is non-zero only when $\nabla\left(\sigma+j \omega \varepsilon_{0} \varepsilon_{\mathrm{r}}\right)$ is non-zero and not tangential to $\nabla \Phi$. The $\nabla\left(\sigma+j \omega \varepsilon_{0} \varepsilon_{\mathrm{r}}\right)$ term is non-zero at the boundary between two different media. The Green's Theorem approach has the advantage that it requires only knowledge of the location and value of internal charge whereas the indirect method requires knowledge of the entire domain. As will be shown, the Green's Theorem approach will significantly reduce the computational complexity compared to the indirect reconstruction method and reduce high prediction errors resulting from measurement noise associated with an ill-posed problem.

The remainder of this chapter is devoted to the development of the Electrical Property Enhanced Tomography (EPET) method. EPET, first introduced by Gregory and Gregory [4] (see Appendix A), applies the charge-charge correlation relationship (equations (3-6) and (3-7)), which was derived from the Green's Theorem expression (equation (3-2)). EPET is related to the existing impedance imaging methods, but does not attempt to create the image with the electrical data. Rather it adds electrical property information to the existing modality and, in fact, requires the data from the other modality to locate the position of internal structures in the object. The charge-charge correlation relationship relates the internal charge within the domain (lower case q) via a sine series to the external charge that builds up on the boundary of the 
domain (uppercase Q). The relationship has two parts. First, the sine transform coefficients, $A_{l}$, are computed from estimates of internal charge, $\delta q$, and a geometric weighting function, $\mathrm{B}(\mathrm{l})$. Second, the coefficients $A_{l}$ are used to compute the external charge buildup, Q. The EPET method uses a priori structural (geometric) information to identify the location of internal charge buildup. This will be discussed later. The buildup is expected to occur at the boundary between different electrical properties per equation (3-5). Using a well defined domain, the chargecharge correlation relationship, and a priori structural information, the EPET method produces estimates of the internal electrical properties within a domain.

$$
\begin{aligned}
A_{l}= & \sum_{i=1}^{N} B_{i}(l) \cdot \delta q_{i} \\
& \text { where: } \\
Q(x)= & \sum_{l=1}^{M} A_{l} \cdot \sin \left(\frac{l \pi x}{a}\right)
\end{aligned}
$$

\subsection{Charge-charge Correlation}

Figure 3-1 presents a rectangular shaped domain that shall be treated as a quasi 2-D domain. The sides (corresponding to the $\mathrm{x}$ and $\mathrm{y}$ axes) of the domain have lengths $\mathrm{a}$ and $\mathrm{b}$, and the height (axis coming out of the paper) is c, corresponding to the $\mathrm{z}$ axis. Voltages are applied along the four sides of the domain $((0, y),(a, y),(x, 0),(x, a))$. The current is measured (sensed) along the $(\mathrm{x}, 0)$ side of the domain. Since this scenario assumes a small height $\mathrm{c}(\mathrm{c}<<\mathrm{a}, \mathrm{b})$ and small variations in electric field (E-field) along the $\mathrm{z}$-direction $\left(\mathrm{E}_{\mathrm{Z}}<<1\right)$, the domain is treated as two-dimensional.

The domain, $\Omega_{1}$, (Figure 3-1) possesses one sub-domain, $\Omega_{2}$, or, alternatively, two media. Medium 1 corresponds to domain $\Omega_{1}$ and boundary $\Gamma_{1}$ while medium 2 corresponds to domain $\Omega_{2}$ with boundary $\Gamma_{2}$. Two coordinate systems, the prime system and unprimed system (corresponding to source and sensed points, respectively), are defined. From equation (3-2), the potential $\Phi(\mathbf{r})$ is determined at the test point $\mathbf{r}$, by the charge density $\rho\left(\mathbf{r}^{\prime}\right)$ at the source point $\mathbf{r}^{\prime}$, by the boundary potential $\Phi\left(\mathbf{r}^{\prime}\right)$, and by the Green’s function, $G_{D}$. 


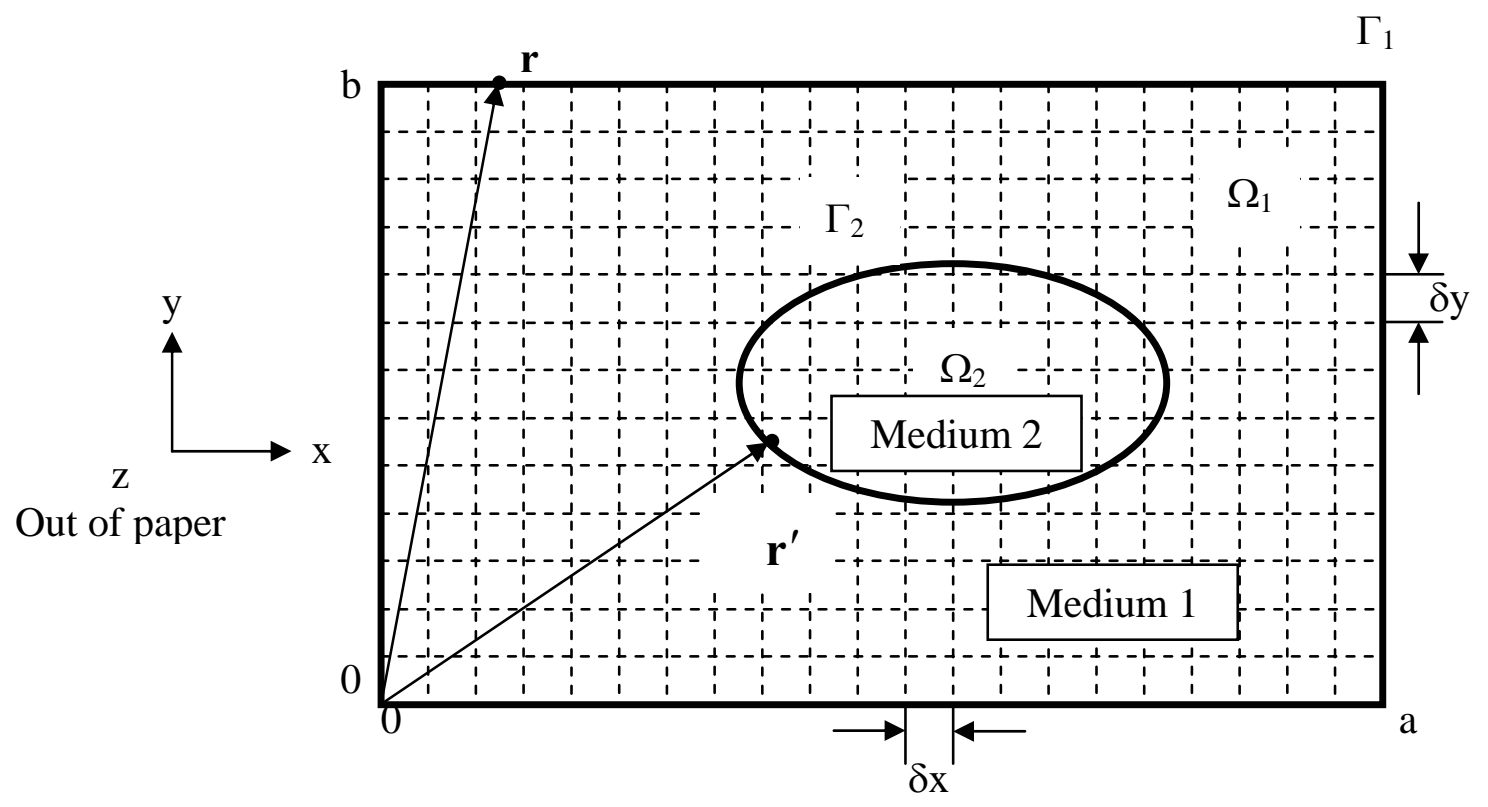

Figure 3-1: A quasi 2D, discretized domain

A two-media, rectangular domain is illustrated. A different medium is contained in each domain $\left(\Omega_{1}\right.$ and $\left.\Omega_{2}\right)$. Two coordinates systems (primed and unprimed) are illustrated. The primed coordinate points to source points and the unprimed coordinate points to sensed points. The domain is discretized with elemental lengths $\delta x$ and $\delta y$.

\subsubsection{Simplifying the Problem}

Consider two scenarios, the uniform and non-uniform cases. In the uniform case, only one medium is in the domain. There are no internal boundaries $\left(\Gamma_{2}\right)$ and thus no internal charges are present. The first term in equation (3-2) is zero resulting in equation (3-8). The potential $\Phi$ has the subscript $\mathrm{u}$ to represent the uniform potential. In the non-uniform case two media are within the domain. Internal charges are present on boundary $\Gamma_{2}$. Equation (3-2) is unchanged (equation (3-9)) and the potential $\Phi$ has the subscript $n$ to represent the non-uniform potential. Taking the difference between the non-uniform and uniform cases (equation (3-10)) eliminates the second term (the surface integral) in the Green’s Theorem, leaving only the volume integral, which includes the charge term (equation (3-11)). The difference potential, $\Delta \Phi$, corresponds to change in the potential field resulting from charge build up at $\Gamma_{2}$. 


$$
\begin{gathered}
\Phi_{u}(\mathbf{r})=\frac{-1}{4 \pi} \oint_{S} \Phi_{S}\left(\mathbf{r}^{\prime}\right) \frac{\partial G_{D}}{\partial n^{\prime}} d a^{\prime} \\
\Phi_{n}(\mathbf{r})=\frac{1}{4 \pi \varepsilon_{0}}\left[\int_{V} \rho\left(\mathbf{r}^{\prime}\right) G_{D}\left(\mathbf{r}, \mathbf{r}^{\prime}\right) d^{3} x^{\prime}-\varepsilon_{0} \oint_{S} \Phi_{S}\left(\mathbf{r}^{\prime}\right) \frac{\partial G_{D}}{\partial n^{\prime}} d a^{\prime}\right] \\
\Delta \Phi(\mathbf{r})=\Phi_{n}(\mathbf{r})-\Phi_{u}(\mathbf{r}) \\
\Delta \Phi(\mathbf{r})=\frac{1}{4 \pi \varepsilon_{0}} \int_{V} \rho\left(\mathbf{r}^{\prime}\right) G_{D}\left(\mathbf{r}, \mathbf{r}^{\prime}\right) d^{3} x^{\prime}
\end{gathered}
$$

\subsubsection{Reducing the Expression to Charge and Potential}

The measured current is related to the internal charge via the current density $\mathbf{J}(\mathbf{x})$ [74] at the boundary of the domain, $\Gamma_{1}$, equation (3-12). The medium ( $\sigma$ and $\varepsilon_{\mathrm{r}}$ ) at the boundary $\Gamma_{1}$ is the same for both the uniform and non-uniform cases.

$$
\mathbf{J}(\mathbf{r})=-\left(\sigma(\mathbf{r})+j \omega \varepsilon_{0} \varepsilon_{r}(\mathbf{r})\right) \nabla \Phi(\mathbf{r})
$$

The current density is a vector quantity. Equation (3-13) relates elemental currents $\left(\delta I_{x}, \delta I_{y}\right)$ passing through elemental areas $(\delta y \delta z, \delta x \delta z)$ at the sense point $\mathbf{r}$ to the current density $\left(\delta J_{x}, \delta J_{y}\right)$.

$$
\mathbf{J}(\mathbf{r})=J_{x}(\mathbf{r}) \hat{\mathbf{x}}+J_{y}(\mathbf{r}) \hat{\mathbf{y}}=\frac{\delta I_{x}(\mathbf{r})}{\delta y \delta z} \hat{\mathbf{x}}+\frac{\delta I_{y}(\mathbf{r})}{\delta x \delta z} \hat{\mathbf{y}}
$$

Equation (3-14) results when equation (3-12) is substituted into equation (3-13) and is evaluated along the $(x, 0)$ side of the domain. Along this side of the domain, the direction of the domain normal points outward, $-\hat{\mathbf{y}}$, the gradient term reduces to the partial derivative with respect to $\mathrm{y}, \frac{\partial}{\partial y} \hat{\mathbf{y}}$, and the elemental area is $\delta x \delta z$. Similar results can be derived for the remaining three sides of the domain.

$$
\delta I_{u}(x, 0)=\mathbf{J}(x, 0) \cdot(-\hat{\mathbf{y}}) \delta x \delta \mathbf{z}=\left(\sigma(x, 0)+j \omega \varepsilon_{0} \varepsilon_{r}(x, 0)\right) \cdot \frac{\partial}{\partial y}(\Delta \Phi(x, 0)) \cdot(-\hat{\mathbf{y}}) \cdot(\delta x \delta z)
$$


Current and charge are related by the law of conservation of charge (equation (3-15)) [74]. The divergence of the current density is related to an elemental current along the $y$ direction in equation (3-16). The charge density function, by separation of variables [74], is divided into positional and time dependent equations (equation (3-17)). The positional charge density is related to the elemental charge, $\delta Q$ (equation (3-18)). Equations (3-16) through (3-18) are substituted into equation (3-15) to create a relationship between the elemental current and elemental charge along the boundary $\Gamma_{1}$. Equation (3-19) along with equation (3-11) and equation (3-12) are substituted in equation (3-14) to produce a relationship between charge on the boundary of the domain, $\delta Q(x, 0)$, and the interior charge density $\rho\left(\mathbf{r}^{\prime}\right)$ (equation (3-20)).

$$
\begin{gathered}
\left.\left(\nabla \cdot \mathbf{J}+\frac{\partial \rho}{\partial t}\right)\right|_{(x, 0)}=0 \\
\left.\nabla \cdot \mathbf{J}\right|_{(x, 0)}=\left.\frac{\partial J_{y}}{\partial y}\right|_{(x, 0)}=\left.\frac{\delta I_{y}}{\delta x \delta y \delta z}\right|_{(x, 0)} \\
\rho(x, 0, t)=\rho(x, 0) \cdot e^{j \omega t} \\
\rho(x, 0)=\frac{\delta Q(x, 0)}{\delta x \delta y \delta z} \\
\delta I(x, 0)=j \omega \delta Q(x, 0) \\
\delta Q_{u}(x, 0)=\frac{j\left(\sigma(x, 0)+j \omega \varepsilon_{0} \varepsilon_{r}(x, 0)\right)}{4 \pi \omega \varepsilon_{0}}\left[\left.\int_{V} \rho\left(\mathbf{r}^{\prime}\right) \cdot \frac{\partial}{\partial y} G_{D}\left(\mathbf{r}, \mathbf{r}^{\prime}\right)\right|_{(x, 0)} d^{3} x^{\prime}\right] \cdot(-\hat{\mathbf{y}}) \cdot(\delta x \delta z)
\end{gathered}
$$

\subsubsection{Discretizing the Formulation}

The domain is discretized (Figure 3-1) with equal lengths of the discretized elements for both the primed and unprimed coordinate systems (equation (3-21)).

$$
\begin{aligned}
& \delta x=\delta x^{\prime} \\
& \delta y=\delta y^{\prime} \\
& \delta z=\delta z^{\prime}=c
\end{aligned}
$$

The volume integral in equation (3-20)) is converted into a summation over a single index, $i$, where the $x^{\prime}$ and $y^{\prime}$ coordinates are mapped to discretized positions $x_{i}{ }_{i}$ and $y^{\prime}{ }_{i}$ (equation $(3-22))$. 


$$
\iiint \rho\left(x^{\prime}, y^{\prime}\right) \frac{\partial G_{D}\left(\mathbf{r}, x^{\prime}, y^{\prime}\right)}{\partial y} d x^{\prime} d y^{\prime} d z^{\prime} \Rightarrow \sum_{i=1}^{\mathrm{N}} \rho\left(x_{i}^{\prime}, y_{i}^{\prime}\right) \frac{\partial G_{D}\left(\mathbf{r}, x_{i}^{\prime}, y_{i}^{\prime}\right)}{\partial y} \delta x^{\prime} \delta y^{\prime} \delta z^{\prime}
$$

The internal charge density (on boundary $\Gamma_{2}$ ) is replaced with an elemental charge per unit volume (equation (3-23)).

$$
\rho\left(\mathbf{r}_{i}^{\prime}\right)=\frac{\delta q\left(x_{i}^{\prime}, y_{i}^{\prime}, z_{i}^{\prime}\right)}{\delta x^{\prime} \delta y^{\prime} \delta z^{\prime}}=\frac{\delta q_{i}}{\delta x^{\prime} \delta y^{\prime} \delta z^{\prime}}
$$

The charge on the boundary of the domain $\left(\Gamma_{1}\right)$ is designated with upper case $\mathrm{Q}$ while the charge in the interior $\left(\Gamma_{2}\right)$ is designated with a lower case q. Both equation (3-22) and equation (3-23) are substituted into equation (3-20) to produce equation (3-24).

$$
\delta Q(x, 0)=\frac{j\left(\sigma+j \omega \varepsilon_{0} \varepsilon_{r}\right)}{4 \pi \omega \varepsilon_{0}} \cdot(\delta x \delta z) \sum_{i=1}^{\mathrm{N}}\left\{\left.\frac{\partial}{\partial y_{i}} \cdot G_{D}\left(\mathbf{r}, \mathbf{r}_{i}^{\prime}\right)\right|_{y=0} \cdot(-\hat{\mathbf{y}})\right\} \delta q_{i}
$$

\subsubsection{Applying the Green's Function for Cartesian Coordinates}

Equation (3-25) is a quasi-2D version of the Green's Function for a rectangular shaped domain and a Cartesian coordinate system adapted from Jackson [74].

$$
G_{D}\left(\mathbf{r}, \mathbf{r}_{i}^{\prime}\right)=\frac{8}{\pi a c} \sum_{l=1}^{M} \frac{\sin \left(\frac{l \pi x}{a}\right) \sin \left(\frac{l \pi x_{i}^{\prime}}{a}\right) \sinh \left(\frac{l \pi}{a} y\right) \sin \left(\frac{l \pi}{a}\left(b-y_{i}^{\prime}\right)\right)}{\frac{l \pi}{a} \sinh \left(\frac{l \pi}{a} b\right)}
$$

Substituting equation (3-25) into equation (3-24) results in equation (3-26).

$$
\begin{aligned}
& \delta Q(x, 0)=\frac{j\left(\sigma+j \omega \varepsilon_{0} \varepsilon_{r}\right)}{4 \pi \omega \varepsilon_{0}} \cdot(\delta x \delta z) \\
& \sum_{i=1}^{\mathrm{N}}\left\{\left.\frac{\partial}{\partial y} \cdot\left[\frac{8}{\pi a c} \sum_{l=1}^{M} \frac{\sin \left(\frac{l \pi x}{a}\right) \cdot \sin \left(\frac{l \pi x_{i}^{\prime}}{a}\right) \cdot \sinh \left(\frac{l \pi}{a} y\right) \sinh \left(\frac{l \pi}{a}\left(b-y_{i}^{\prime}\right)\right)}{\frac{l \pi}{a} \sinh \left(\frac{l \pi}{a} b\right)}\right]\right|_{(x, 0)} \cdot(-\hat{\mathbf{y}})\right\} \delta q_{i}
\end{aligned}
$$




\subsubsection{Using Sine Transforms to Produce the Charge-charge Correlation}

\section{Relationship}

The $\sin \left(\frac{l \pi x}{a}\right)$ term in equation (3-26) is eliminated by applying a sine transform (equation (3-27)) to both sides of the equation. The result is equation (3-28). The variable $\mathrm{A}_{l}$ is the $l^{\text {th }}$ sine transform coefficient in the sine series approximation of the boundary charge distribution, $\mathrm{Q}(\mathrm{x}, 0)$.

$$
\begin{gathered}
S T\{\delta Q(x, 0), l\}=\mathrm{A}_{i}=\frac{2}{a} \int_{0}^{a} \delta Q(x, 0) \sin \left(\frac{l \pi x}{a}\right) d x \\
\mathrm{~A}_{l}=-\frac{j\left(\sigma+j \omega \varepsilon_{0} \varepsilon_{r}\right)}{4 \pi \omega \varepsilon_{0}} \delta x \delta z \sum_{i=1}^{\mathrm{N}}\left\{\left.\frac{8 \pi}{a c} \sin \left(\frac{l \pi x_{i}^{\prime}}{a}\right) \frac{\partial}{\partial y}\left[\frac{\sinh \left(\frac{l \pi}{a} y\right) \sinh \left(\frac{l \pi}{a}\left(b-y_{i}^{\prime}\right)\right)}{\frac{l \pi}{a} \sinh \left(\frac{l \pi}{a} b\right)}\right]\right|_{(x, 0)}\right\} \delta q_{i}
\end{gathered}
$$

Finally, the partial derivative with respect to the y coordinate is applied in equation (3-28) evaluated at $(x, 0)$ to produce equation (3-29), the charge-charge correlation relationship.

$$
\mathrm{A}_{l}=-2 \frac{j\left(\sigma+j \omega \varepsilon_{0} \varepsilon_{r}\right)}{\omega \varepsilon_{0}}\left(\frac{\delta x \delta z}{a c}\right) \sum_{i=1}^{N}\left\{\sin \left(\frac{l \pi x_{i}^{\prime}}{a}\right) \frac{\sinh \left(\frac{l \pi}{a}\left(b-y^{\prime}\right)\right)}{\sinh \left(\frac{l \pi}{a} b\right)}\right\} \delta q_{i}
$$

Equation (3-29) is converted into a matrix expression (equation (3-30)), where the elements of the geometric matrix, $\mathrm{B}_{\mathrm{i}}(\mathrm{l})$, are defined in equation (3-31). The surface charge, $\mathrm{Q}(\mathrm{x}, 0)$, is computed from the sine series by using the sine transform coefficient, $\mathrm{A}_{l}$ (equation (3-32)).

$$
\begin{aligned}
& \left(\begin{array}{c}
\mathrm{A}_{1} \\
\mathrm{~A}_{2} \\
\vdots \\
\mathrm{A}_{M}
\end{array}\right)=-2 \frac{j\left(\sigma+j \omega \varepsilon_{0} \varepsilon_{r}\right)}{\omega \varepsilon_{0}}\left(\frac{\delta x \delta z}{a c}\right)\left(\begin{array}{cccc}
\mathrm{B}_{1}(1) & \mathrm{B}_{2}(1) & \cdots & \mathrm{B}_{\mathrm{N}}(1) \\
\mathrm{B}_{1}(2) & \ddots & & \\
\vdots & & & \\
\mathrm{B}_{1}(\mathrm{M}) & & & \mathrm{B}_{\mathrm{N}}(\mathrm{M})
\end{array}\right)\left(\begin{array}{c}
\delta q_{1} \\
\delta q_{2} \\
\vdots \\
\delta q_{\mathrm{N}}
\end{array}\right) \\
& \mathrm{B}_{i}(l)=\sin \left(\frac{l \pi x_{i}^{\prime}}{a}\right) \frac{\sinh \left(\frac{l \pi}{a}\left(b-y^{\prime}\right)\right)}{\sinh \left(\frac{l \pi}{a} b\right)}
\end{aligned}
$$




$$
\delta Q(x)=\sum_{l=1}^{M} A_{l} \sin \left(\frac{l \pi x}{a}\right)
$$

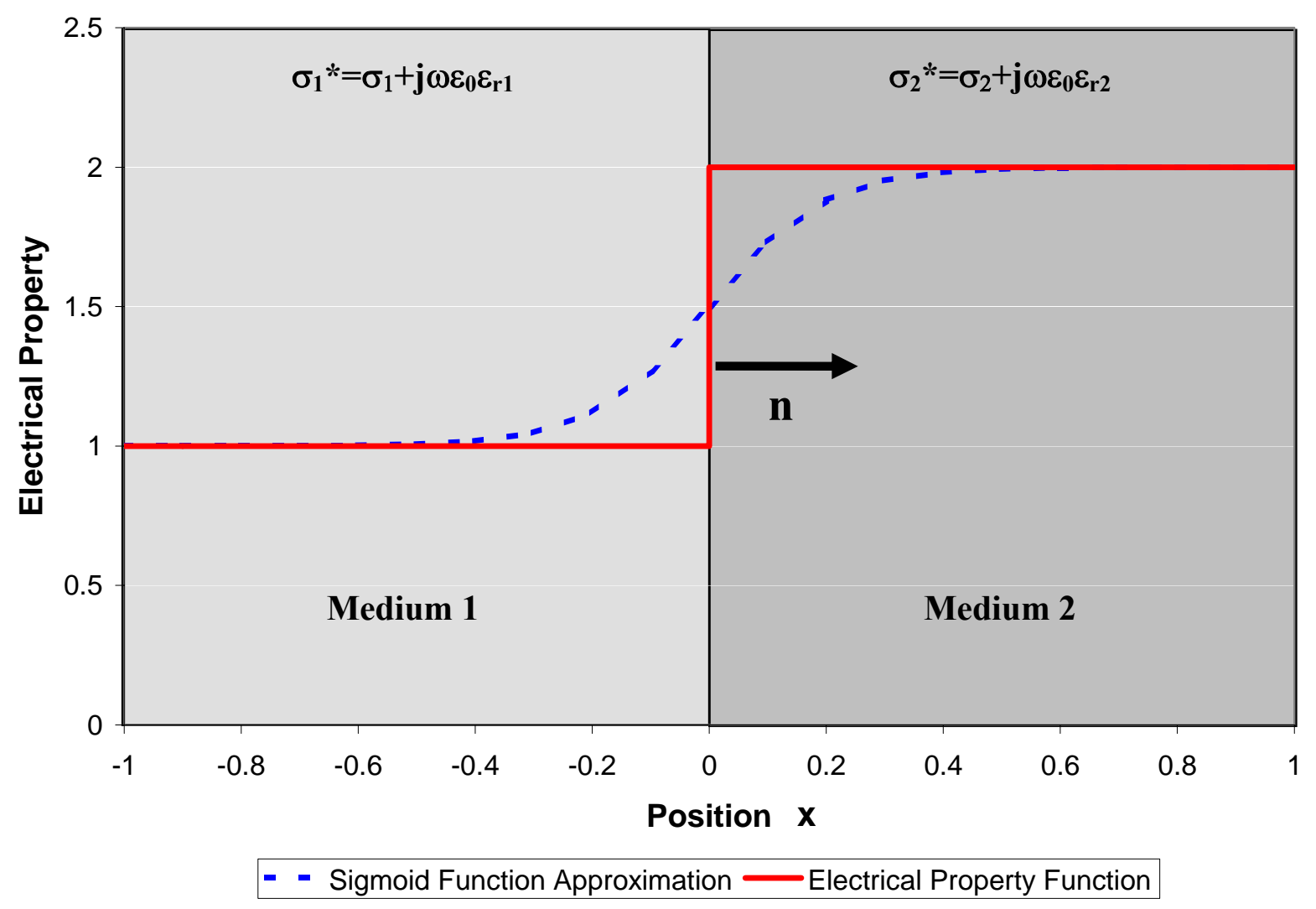

Figure 3-2: Representation of electrical properties at internal boundaries

A two-media domain with a boundary located at $x=0$ is illustrated. As a function of position, the electrical properties are represented as a step function (solid line) and approximated with a sigmoid function (dashed line). The vector perpendicular (normal) to the boundary is also illustrated.

\subsection{Relating Internal Charge to Electrical Property Values}

The internal charge terms in equation (3-30) must be replaced with expressions involving the internal electrical properties. To address this issue consider equation (3-5) in conjunction with Figure 3-2, which illustrates a case with two media, A and B, each possessing different symbolic electrical properties (magnitude of 1 for medium A and 2 for medium B).

Charge is deposited on the boundary surface between the two media, thus the charge density is effectively a charge per unit area per unit of thickness, $\Delta$ (equation (3-33)), where the 
thickness is a transitional distance between the two media where the charge accumulates. The properties change as a step function at the interface, $x=0$, represented by a solid line. It is desirable to model this step function with a continuous function that has a continuous first derivative. The sigmoid function (dashed line) [148] is one such function commonly used to model step function changes (equation (3-34)). The gradient of the electrical properties (equation (3-3)) is evaluated at the boundary $(\mathrm{x}=0)$ and produces an expression of the charge density (and thus the charge buildup) that is proportional to a function involving the electrical properties (equation (3-35)).

One last definition, the contrast ratio, is introduced. The contrast ratio is the ratio of electrical properties that meet at a common boundary. In this example, the contrast ratio, $\kappa$, is defined in equation (3-36), substituted into equation (3-35) to produce equation (3-37).

$$
\rho=\frac{\delta q}{\delta \text { area } \cdot \Delta}
$$

where $\Delta$ is a transition distance (thickness) where charge builds up)

$$
\sigma^{*}(x)=\frac{\sigma_{B}{ }^{*}-\sigma_{A}{ }^{*}}{1+e^{-x / \Delta}}+\sigma_{A}^{*}
$$

where $\sigma_{\mathrm{A}} *$ and $\sigma_{\mathrm{B}} *$ are the complex conductivities in media $\mathrm{A}$ and $\mathrm{B}$

$$
\delta q=\left(\frac{\sigma_{B}{ }^{*}-\sigma_{A}{ }^{*}}{{\sigma_{B}}^{*}+\sigma_{A}{ }^{*}}\right)\left(\frac{-\varepsilon_{0} \delta \text { sarea }}{2}\right) \hat{\mathbf{x}} \cdot \nabla \Phi
$$

where $\hat{\mathbf{x}}$ is the unit vector normal to the boundary

$$
\begin{gathered}
\kappa=\frac{\sigma_{B}^{*}}{\sigma_{A}^{*}} \\
\delta q=\left(\frac{\kappa-1}{\kappa+1}\right)\left(\frac{-\varepsilon_{0} \delta a r e a}{2}\right) \hat{\mathbf{x}} \cdot \nabla \Phi
\end{gathered}
$$

\subsection{Electrical Property Enhanced Tomography}

The ultimate goal in EIT is to determine value(s) of the electrical properties within a medium. The charge-charge correlation formula (equation (3-30) to (3-32)) provides a methodology to relate internal charge build-up ( $\left.\delta q_{i}\right)$ to external charge patterns $(Q(x))$. The sigmoid function was used to compute the internal charge build-up at the boundaries between media (equation (3-37)). In order to apply equation (3-30), it is necessary to determine the

location of the media boundaries $\left(x_{i}, y_{i}\right)$ and the local gradient of the potential field $\left(\left.\nabla \Phi\right|_{x_{i}, y_{i}}\right)$. 
To determine the boundary locations, a priori positional information, typically from a different imaging modality is used. In a clinical setting, this second image might be an MR or CT image. The local gradient of the potential field must be approximated.

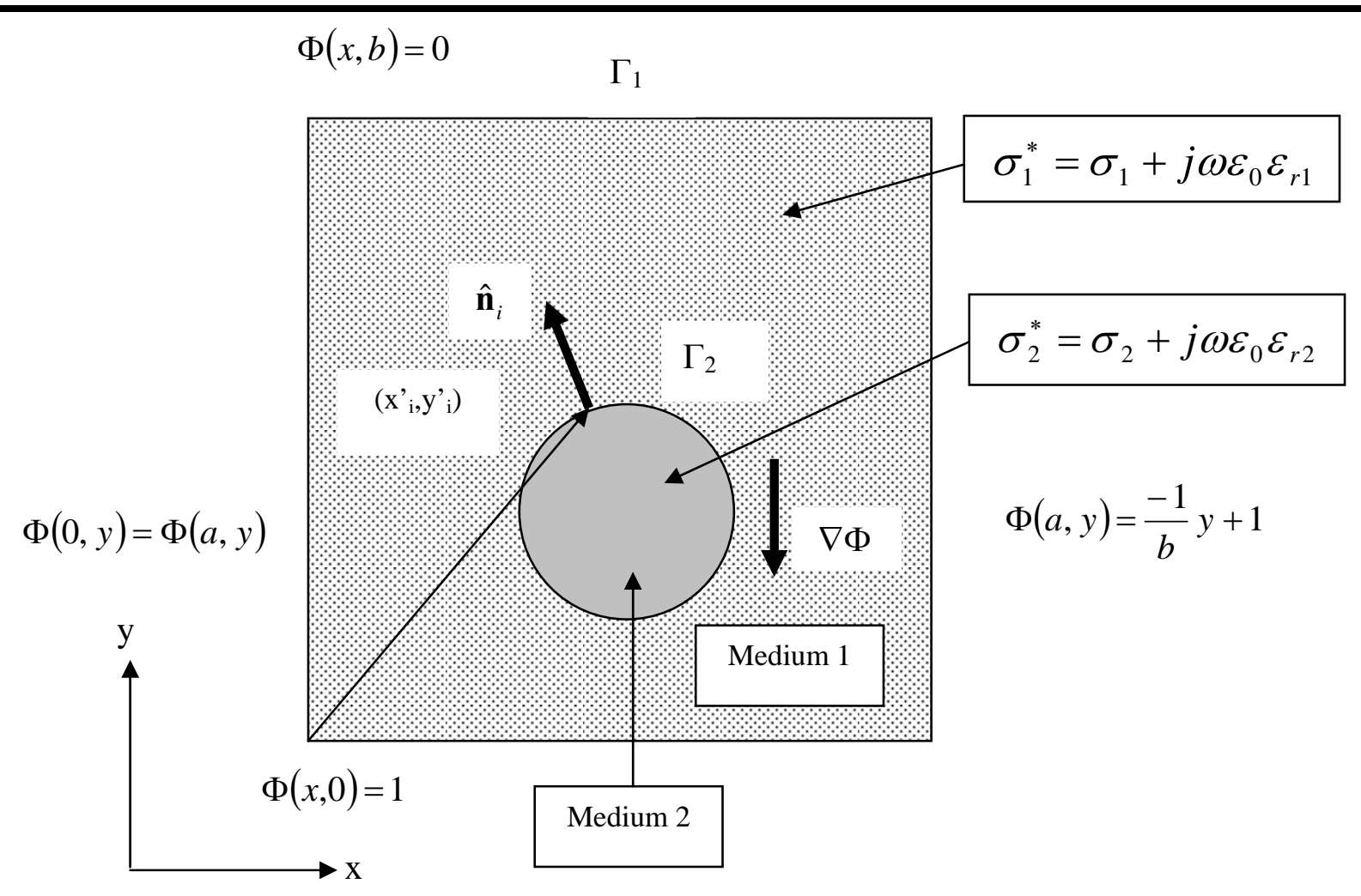

Figure 3-3: Domain with two media

A two-media, rectangular domain with Dirichlet boundary conditions is illustrated. Electrical properties, $\sigma_{1}{ }^{*}$ and $\sigma_{2}{ }^{*}$, and the boundary potential, $\Phi$, are defined. At an arbitrary point, $\mathrm{x}_{\mathrm{i}}{ }^{\prime}$ and $\mathrm{y}_{\mathrm{i}}^{\prime}$, along the boundary $\Gamma_{2}$, the vector normal to the boundary is defined. The potential gradient is also defined.

Consider Figure 3-3 that represents a domain with two media. The internal charge $\delta q_{i}$ is located at coordinates $\left(\mathrm{x}_{\mathrm{i}}, \mathrm{y}_{\mathrm{i}}\right)$. The electrical properties are $\sigma_{1}^{*}$ and $\sigma_{2}^{*}$ for media 1 and 2 respectively. The applied boundary potential is $\Phi_{s}=1$ along the $(\mathrm{x}, 0)$ side, $\Phi_{s}=0$ along the (x,b) side and varies linearly $\left(\Phi_{s}=\frac{-1}{b} y+1\right)$ along the $(0, y)$ and $(\mathrm{a}, \mathrm{y})$ sides. The unit vector $\hat{\mathbf{n}}$ replaces $\hat{\mathbf{x}}$ in equation (3-35).

To compute the gradient of the potential in the non-uniform case, the potential is approximated using the uniform case. The uniform case is trivial and is solved using finite 
difference methods. The solution is then fitted to a $4^{\text {th }}$ order polynomial (equation (3-38)) where the coefficients $\mathrm{kx}(\mathrm{i}, \mathrm{j})$ are listed in equation (3-39). The gradient of the potential is computed directly from the polynomial fit to yield equation (3-40). The reader is directed to Appendix B for additional detail regarding the computation and polynomial fitting of the uniform potential.

$$
\Phi(x, y)=\sum_{i=1}^{6} \sum_{j=1}^{6} k x(i, j) \cdot x^{i} \cdot y^{j}
$$

where:

$$
k x=\left(\begin{array}{cccccc}
9.88 \mathrm{E}-01 & -1.43 \mathrm{E}-02 & -1.14 \mathrm{E}-04 & 5.63 \mathrm{E}-06 & -7.05 \mathrm{E}-08 & 2.99 \mathrm{E}-10 \\
1.17 \mathrm{E}-03 & -1.36 \mathrm{E}-05 & 1.33 \mathrm{E}-05 & -4.94 \mathrm{E}-07 & 6.39 \mathrm{E}-09 & -2.83 \mathrm{E}-11 \\
-5.11 \mathrm{E}-05 & 5.21 \mathrm{E}-06 & -5.89 \mathrm{E}-07 & 1.93 \mathrm{E}-08 & -2.47 \mathrm{E}-10 & 1.09 \mathrm{E}-12 \\
7.93 \mathrm{E}-07 & -1.07 \mathrm{E}-07 & 9.57 \mathrm{E}-09 & -3.03 \mathrm{E}-10 & 3.86 \mathrm{E}-12 & -1.71 \mathrm{E}-14 \\
-3.55 \mathrm{E}-09 & 5.16 \mathrm{E}-10 & -5.05 \mathrm{E}-11 & 1.64 \mathrm{E}-12 & -2.11 \mathrm{E}-14 & 9.39 \mathrm{E}-17 \\
-6.05 \mathrm{E}-12 & 4.86 \mathrm{E}-13 & -9.87 \mathrm{E}-15 & 4.72 \mathrm{E}-18 & 1.39 \mathrm{E}-18 & -8.85 \mathrm{E}-21
\end{array}\right)
$$

$$
\nabla \Phi(x, y)=\sum_{i=0}^{5} \sum_{j=0}^{5} i \cdot k x(i, j) \cdot x^{i-1} \cdot y^{j} \hat{\mathbf{i}}+\sum_{i=0}^{5} \sum_{j=0}^{5} j \cdot k x(i, j) \cdot x^{i} \cdot y^{j-1} \hat{\mathbf{j}}
$$

Substituting equations (3-37) and (3-40) into (3-30) produces (3-41) (recalling that $\delta z=c$ for the quasi-2D approximation). The coefficient $A_{l}$ is simplified by separating the product of the $\frac{\kappa-1}{\kappa+1}$ term from the other terms (equation (3-42)) to produce $A^{\prime}{ }_{1}$ (equation (3-43)). When medium 2 is composed of metal, the contrast ratio is very large $\left(\sigma_{2}^{*}>>\sigma_{1}^{*}\right)$. The first term in equation (3-42) is approximately $1\left(\frac{\sigma_{2}^{*}-\sigma_{1}^{*}}{\sigma_{2}^{*}+\sigma_{1}^{*}} \approx 1\right)$. Consequently, the coefficient $\mathrm{A}^{\prime}$ l produces the charge pattern, $\mathrm{Q}(\mathrm{x}, 0)$, for a high contrast ratio.

$$
\begin{gathered}
\mathrm{A}_{l}=\frac{\kappa-1}{\kappa+1}\left\{-\left(\sigma_{1}^{*} \frac{\delta x}{a}\right) \sum_{i=1}^{\mathrm{N}} \mathrm{B}_{i}(l) \cdot\left(\text { sarea }\left._{i} \cdot \hat{\mathbf{n}}_{i} \cdot \nabla \Phi\right|_{\left(x_{i}, y_{i}\right)}\right)\right\} \\
\mathrm{A}_{l}=\frac{\kappa-1}{\kappa+1} A_{l}^{\prime} \\
\mathrm{A}_{l}^{\prime}=-\left(\sigma_{1}^{*} \frac{\delta x}{a}\right) \sum_{i=1}^{\mathrm{N}} \mathrm{B}_{i}(l) \cdot\left(\text { darea }\left._{i} \cdot \hat{\mathbf{n}}_{i} \cdot \nabla \Phi\right|_{\left(x_{i}, y_{i}\right)}\right)
\end{gathered}
$$




\subsubsection{EPET Forward Solution}

In an experimental application, the domain is lined with electrodes at the boundary $\Gamma_{1}$. The potential is applied at each electrode and the current, $I_{k}^{m}$, that passes through each electrode $\mathrm{k}$ (located at position $\mathrm{x}_{\mathrm{k}}$ and $\mathrm{y}=0$ ), is measured. The predicted current, $I_{k}^{p}$, is computed by summing a sine series (with coefficients $\mathrm{A}^{\prime}{ }_{l}$ ) over a fixed number of terms, $l_{\max }$ (equation (3-44)). Since the summation term in (3-44) possesses current units, it corresponds to the current pattern $\kappa>>1$ and is referred to as the 'high contrast forward solution' throughout the remainder of the dissertation (equation (3-45)).

$$
\begin{gathered}
I^{p}\left(x_{k}, 0\right)=I_{k}^{p}=\frac{\kappa-1}{\kappa+1} \cdot \sum_{l=1}^{l_{\max }} A_{l}^{\prime} \cdot \sin \left(\frac{l \pi x_{k}}{a}\right) \\
I^{\prime p}\left(x_{k}, 0\right)=\sum_{l=1}^{l_{\max }} A_{l}^{\prime} \cdot \sin \left(\frac{l \pi x_{k}}{a}\right)
\end{gathered}
$$

\subsubsection{EPET Inverse Solution}

The current pattern, $\mathbf{I}^{\mathrm{p}}$, (equation (3-46)) is represented by a column vector, which for a sequence of 15 electrodes results in a 1 column by 15 rows vector. Each element of the vector corresponds to a current reading at each individual electrode. The measured current pattern, $\mathbf{I}^{\mathrm{m}}$, is proportional in magnitude to the high contrast forward solution $\mathbf{I}^{\mathrm{p}}$ (equation (3-47)). Using a least-squares regression fit, the scaling factor $\xi$ is determined. The electrical properties are

computed by equating $\xi$ with $\frac{\kappa-1}{\kappa+1}$ (equation (3-48)) and solving for the unknown contrast ratio, $\kappa$ (equation (3-49)). The remainder of this dissertation tests the EPET method for its accuracy and computational speed.

$$
\begin{gathered}
\mathbf{I}^{p}=\left(\begin{array}{c}
I_{1}^{p} \\
I_{2}^{p} \\
\vdots \\
I_{15}^{p}
\end{array}\right) \\
\mathbf{I}^{m}=\xi \cdot \mathbf{I}^{p}
\end{gathered}
$$




$$
\begin{gathered}
\xi=\frac{\kappa-1}{\kappa+1} \\
\kappa=\frac{1+\xi}{1-\xi}
\end{gathered}
$$




\section{MATERIALS AND METHODS}

This chapter describes experiments and methods used in testing the EPET concept. The chapter is divided into six sections. The first section describes the experiments, next the numerical methods are reviewed, the media preparation is described, the process for determining internal boundary locations is explained, experimental procedures are listed, and finally the experimental set-up is described.

\subsection{Description of Experiments}

The experiments described below are divided into nine experimental groups. Experiments using two media (Figure 4-1) are described in experimental groups 1 through 6 while experiments using three media (Figure 4-2) are described in experimental groups 7 through 9.

\subsubsection{Two-Media Experiments}

Figure 4-1 presents the arrangement and defines key variables for two media experiments. A square tank (23.2 $\mathrm{cm}$ on a side) is filled with medium 1 (a liquid) while medium 2 (a solid) is placed at a known location within the tank. A potential, $\Phi_{\mathrm{S}}$, is applied at a frequency of $50 \mathrm{kHz}$ along the boundary of medium 1 . This potential is 1 Vrms along the $(\mathrm{x}, 0)$ side of the domain, 0 Vrms along the $(\mathrm{x}, 23.2)$ side of the domain, and varies linearly along the $(0, y)$ and $(23.2, y)$ sides of the tank.

Medium 1 is in all cases a saline bath of known conductivity, $\sigma_{1}$. Medium 2, with conductivity $\sigma_{2}$, is a cylinder with a diameter $\mathrm{d}_{2}$ composed of either an agar doped with salt or

stainless steel. The contrast ratio, $\kappa_{21}=\frac{\sigma_{2}}{\sigma_{1}}$, for agar ranges from approximately 1 to 20 while for stainless steel the contrast ratio is approximately $10^{7}$. The lateral and depth position of the center of medium 2 are represented by the position $\mathrm{x}_{2}$ and $\mathrm{y}_{2}$ respectively. Collectively, the position, size (diameter) and contrast ratio of the medium $2\left(\mathrm{x}_{2}, \mathrm{y}_{2}, \mathrm{~d}_{2}\right.$, and $\left.\kappa_{21}\right)$ describe the 'state' of the experiment.

Experimental groups 1 though 4 listed below describe experiments that test the behavior of one of the four descriptive variables while leaving the remaining three variables unchanged. 
Experimental groups 5 and 6 describe experiments that will test the accuracy of the EPET method for predicting contrast values while varying the depth and size respectively. These experiments are performed using only agar for medium 2 that possess a family of five different contrast values.

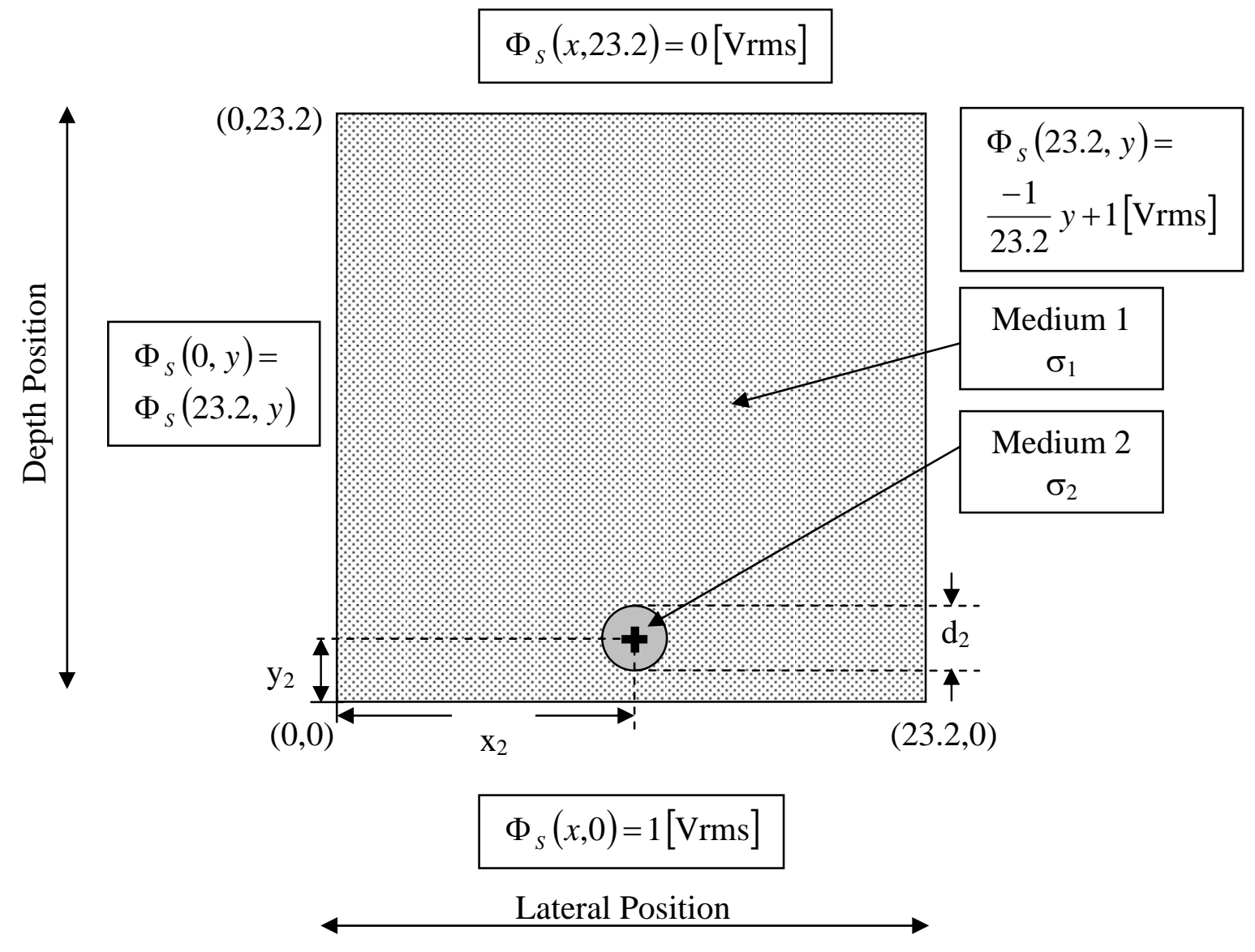

Figure 4-1: Two Media Case

A two-media, rectangular domain with Dirichlet boundary conditions is illustrated. Electrical properties, $\sigma_{1}{ }^{*}$ and $\sigma_{2}{ }^{*}$, and the boundary potential, $\Phi$, are defined. The center of mass, $\mathrm{x}_{2}$ and $\mathrm{y}_{2}$, as well as the diameter, $\mathrm{d}_{2}$, for medium 2 are defined.

Table 4-1 summarizes the state information for each group of experiments. Each row of the table lists the state for a given experimental group. The variable in each experimental group that is varied is highlighted. The experimental groups are summarize below:

Experimental Group 1: Variation of lateral position of Medium 2

Medium 2 is placed in fourteen different lateral positions ranging from $\mathrm{x}_{2}=1.7$ to 11.6 $\mathrm{cm}$.

Experimental Group 2: Variation of depth position of Medium 2

Medium 2 is placed in six different depth positions ranging from $\mathrm{y}_{2}=2.4$ to $11.6 \mathrm{~cm}$. 
Table 4-1: Parameters used experimental groups 1 through 6

\begin{tabular}{|c|c|c|c|c|}
\hline $\begin{array}{l}\text { Experimental } \\
\text { Group }\end{array}$ & $\begin{array}{c}\text { Lateral Position } \\
\quad \mathrm{x}_{2}(\mathrm{~cm})\end{array}$ & $\begin{array}{c}\text { Depth Position } \\
\mathrm{y}_{2}(\mathrm{~cm})\end{array}$ & $\begin{array}{c}\text { Size } \\
\mathrm{d}_{2}(\mathrm{~cm})\end{array}$ & $\begin{array}{c}\text { Contrast Ratio } \\
\kappa_{21}=\frac{\sigma_{2}}{\sigma_{1}}\end{array}$ \\
\hline 1 & $\begin{array}{l}1.7,2.4,3.2,3.9 \text {, } \\
4.7,5.5,6.2,7.0 \text {, } \\
7.7,8.5,9.3 \text {, } \\
10.0,10.8 \text {, and } \\
11.6\end{array}$ & 2.4 & 1.9 & $10^{7}$ \\
\hline 2 & 11.6 & $\begin{array}{l}\text { 2.4, 3.9, 5.5, 7.0, } \\
8.5 \text {, and 11.6 }\end{array}$ & 1.9 & $10^{7}$ \\
\hline 3 & 11.6 & 2.4 & $\begin{array}{l}1.0,1.3,1.6 \text { and } \\
1.9\end{array}$ & $10^{7}$ \\
\hline 4 & 11.6 & 2.4 & 1.9 & $\begin{array}{l}\text { 0.86, 1.11, 1.28, } \\
1.44,1.65,1.80, \\
1.99,2.15,2.26, \\
2.56,2.68,2.73, \\
3.34,3.36,4.06, \\
4.07,4.17,5.23, \\
5.23,6.33,6.84, \\
8.53,10.7, \text { and } \\
12.9\end{array}$ \\
\hline 5 & 11.6 & $\begin{array}{l}\text { 2.4, 3.9, 5.5, 7.0, } \\
8.5 \text {, and } 11.6\end{array}$ & 1.9 & $\begin{array}{l}1.7,2.4,4.4,7.4 \\
\text { and } 15.2\end{array}$ \\
\hline 6 & 11.6 & 2.4 & $\begin{array}{l}1.0,1.3,1.6 \text { and } \\
1.9\end{array}$ & $\begin{array}{l}1.6,2.3,4.2,6.9 \\
\text { and } 13.1\end{array}$ \\
\hline
\end{tabular}

Experimental Group 3: Variation of size of Medium 2

Medium 2 has four different sizes ranging from $\mathrm{d}_{2}=1.0$ to1.9 $\mathrm{cm}$.

Experimental Group 4: Variation of contrast of Medium 2

Medium 2 has 24 different contrast ratio values ranging from $\kappa_{21}=0.86$ to 12.9. To

achieve this variation, four different sample conductivity values $\left(\sigma_{2}=0.277,0.412,0.641\right.$, and

$1.309 \mathrm{~S} / \mathrm{m})$ and six different bathwater conductivity values $\left(\sigma_{1}=0.323,0.250,0.192,0.153\right.$,

0.123 , and $0.101 \mathrm{~S} / \mathrm{m}$ ) are used producing 24 separate possible combinations of contrast values.

\section{Experimental Group 5: Variation of depth and contrast of Medium 2}

Medium 2 has five different contrast values (ranging from $\kappa_{21}=1.7$ to 15.2 ) placed at six

different depth positions (ranging $\mathrm{y}_{2}=2.4$ to $11.6 \mathrm{~cm}$ ).

\section{Experimental Group 6: Variation of size and contrast of Medium 2}

Medium 2 has five different contrast values (ranging from $\kappa_{21}=1.6$ to 13.1) and four

different sizes (ranging from $\mathrm{d}_{2}=1.0$ to $1.9 \mathrm{~cm}$ ). 


\subsubsection{Three Media Experiments}

Figure 4-2 presents the arrangement and defines key variables for three media experiments. In these experiments, medium $3\left(\sigma_{3}\right)$ in embedded in medium $2\left(\sigma_{2}\right)$ that is placed in medium $1\left(\sigma_{1}\right)$, a saline bath. The position, size, and contrast of media 2 and 3 collectively are defined as $\left(\mathrm{x}_{2}, \mathrm{y}_{2}, \mathrm{~d}_{2}\right.$, and $\left.\kappa_{21}\right)$ and $\left(\mathrm{x}_{3}, \mathrm{y}_{3}, \mathrm{~d}_{3}\right.$, and $\left.\kappa_{32}\right)$, respectively. The contrast ratios are

defined as $\kappa_{21}=\frac{\sigma_{2}}{\sigma_{1}}$ and $\kappa_{32}=\frac{\sigma_{3}}{\sigma_{2}}$. The applied boundary potential is the same as in the two media experiments.

Three experimental groups, 7 through 9, are described below. In experimental groups 7 and 8, medium 2 is composed of agar and medium 3 is composed of either agar or stainless steel. As in the two media experiments, the agar is doped with salt and the contrast ratios ranged from 1.4 to 11.7. These experimental groups test the accuracy of the EPET method for predicting contrast values for the more complex three media case while varying the depth and size respectively. These experiments are performed using medium 3 that possess a family of four different contrast values.

Experimental group 9 departs from previous experiments by using different materials. In this case, medium 2 is composed of freshly slaughtered liver and medium 3 is composed of an oatmeal and salt mixture. Liver is selected to represent biological media. The conductivity and relative permittivity of liver is approximately $0.14 \mathrm{~S} / \mathrm{m}$ and 15000 respectively. By contrast, the conductivity and relative permittivity of agar is approximately $0.14 \mathrm{~S} / \mathrm{m}$ and 80 respectively. This experimental group explores how well the EPET method functions when the test media possess high relative permittivity values typically found in biological media. 


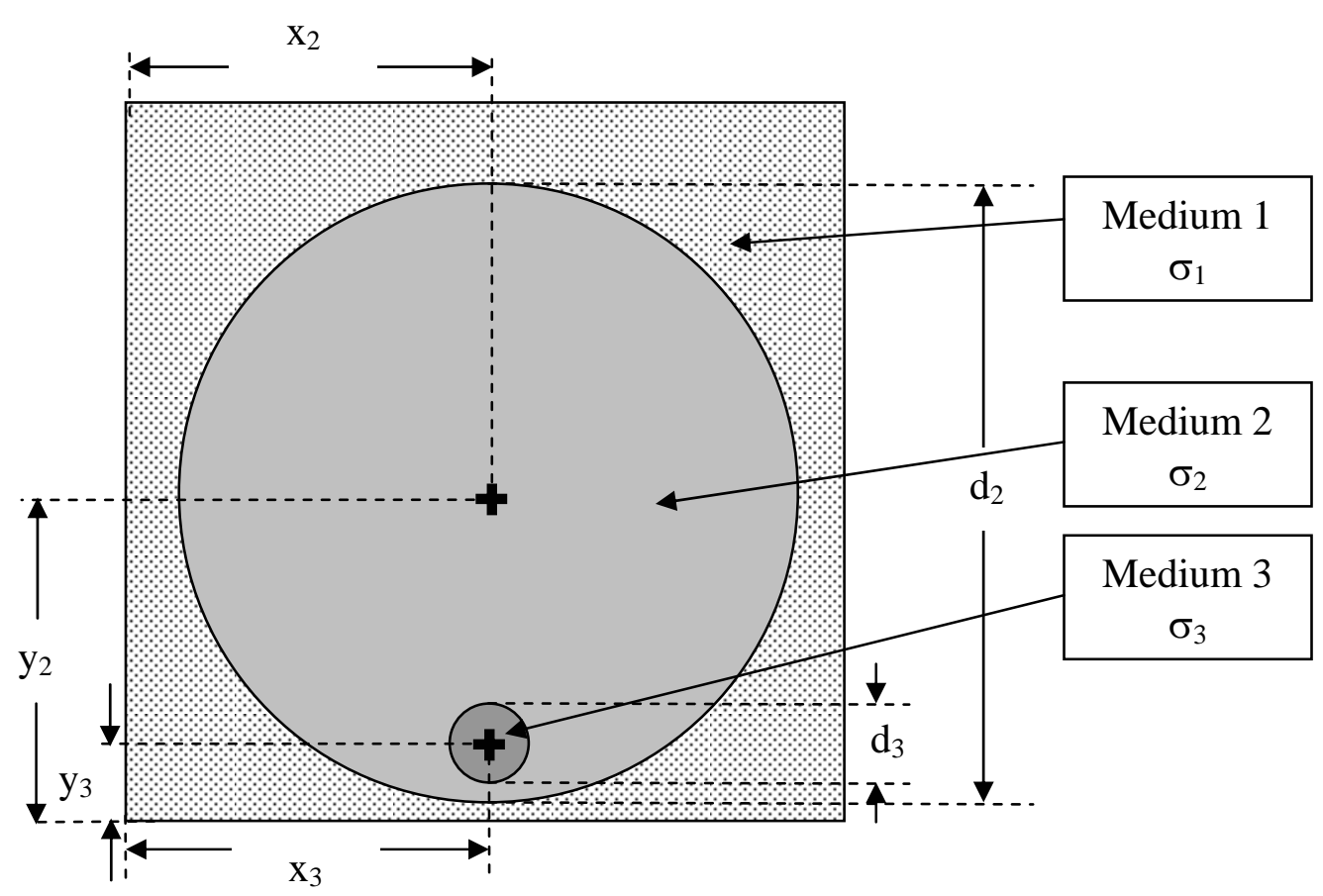

Figure 4-2: Three Media Case

A three-media, rectangular domain with Dirichlet boundary conditions is illustrated. Electrical properties, $\sigma_{1}{ }^{*}, \sigma_{2}{ }^{*}$ and $\sigma_{3}{ }^{*}$, and the boundary potential, $\Phi$, are defined. The centers of mass, $\mathrm{x}_{2}$, $\mathrm{y}_{2}, \mathrm{x}_{3}$, and $\mathrm{y}_{3}$, as well as the diameters, $\mathrm{d}_{2}$ and $\mathrm{d}_{3}$, for medium 2 and 3 are defined.

Table 4-2 summarizes the state information for experimental groups 7 and 8 . The experimental groups are summarize below:

Experimental Group 7: Variation of depth and contrast of Medium 3

Medium 3 has four different contrast values (ranging from $\kappa_{32}=1.4$ to 11.7) placed at four different depth positions (ranging from $\mathrm{y}_{3}=2.8$ to $7.8 \mathrm{~cm}$ ). The lateral position and size $\left(\mathrm{x}_{3}=11.6 \mathrm{~cm}\right.$ and $\left.\mathrm{d}_{3}=1.3 \mathrm{~cm}\right)$ remain unchanged. The state of media 1 and 2 remain unchanged $\left(\mathrm{x}_{2}=11.6 \mathrm{~cm}, \mathrm{y}_{2}=11.4 \mathrm{~cm}, \mathrm{~d}_{2}=20.3 \mathrm{~cm}, \sigma_{1}=0.1 \mathrm{~S} / \mathrm{m}\right.$ and $\left.\sigma_{2}=0.14 \mathrm{~S} / \mathrm{m}\right)$.

\section{Experimental Group 8: Variation of size and contrast of Medium 3}

Medium 3 has four different contrast values (ranging from $\kappa_{32}=1.4$ to 11.7) and four different sizes (ranging from $\mathrm{d}_{3}=1.0$ to $\left.1.9 \mathrm{~cm}\right)$. The lateral position and depth $\left(\mathrm{x}_{3}=11.6 \mathrm{~cm}\right.$ and $\left.\mathrm{y}_{3}=2.8 \mathrm{~cm}\right)$ remain unchanged. The state of media 1 and 2 remain unchanged $\left(\mathrm{x}_{2}=11.6 \mathrm{~cm}, \mathrm{y}_{2}=\right.$ $11.4 \mathrm{~cm}, \mathrm{~d}_{2}=20.3 \mathrm{~cm}, \sigma_{1}=0.1 \mathrm{~S} / \mathrm{m}$ and $\left.\sigma_{2}=0.14 \mathrm{~S} / \mathrm{m}\right)$.

\section{Experimental Group 9: High relative permittivity for Medium 2 with time varying conditions}

This experimental group was performed under nominally constant conditions nineteen times over a 17 hour period. The position and size of media 2 and 3 remain unchanged $\left(\mathrm{x}_{2}=\right.$ 
$\left.11.6 \mathrm{~cm}, \mathrm{y}_{2}=11.4 \mathrm{~cm}, \mathrm{~d}_{2}=20.3 \mathrm{~cm}, \mathrm{x}_{3}=11.6 \mathrm{~cm}, \mathrm{y}_{3}=2.8 \mathrm{~cm}, \mathrm{~d}_{3}=1.3\right)$. The electrical properties of two media vary over time. The conductivity of medium 1 ranges from 0.1 to $0.13 \mathrm{~S} / \mathrm{m}$ for each experimental run. This variation is due to the mixing of blood from the liver (medium 2) with the saline bath (medium 1). Over a 17 hour period, the conductivity of the medium 2 (liver) increases from approximately 0.11 to $0.14 \mathrm{~S} / \mathrm{m}$ while the relative permittivity decreases from approximately from 14500 to 9500 . The conductivity of medium 3 remains unchanged $\left(\sigma_{3}=\right.$ $0.25 \mathrm{~S} / \mathrm{m})$. Unlike all other experimental groups that apply the charge-charge correlation method, this experimental group is solved using the indirect reconstruction method (discussed below).

Table 4-2: Parameters used experimental groups 7 and 8

\begin{tabular}{|l||l|l|l|}
\hline \multicolumn{1}{|c||}{$\begin{array}{c}\text { Experimental } \\
\text { Group }\end{array}$} & $\begin{array}{c}\text { Depth Position } \\
\mathrm{y}_{3}(\mathrm{~cm})\end{array}$ & \multicolumn{1}{c|}{$\begin{array}{c}\text { Size } \\
\mathrm{d}_{3}(\mathrm{~cm})\end{array}$} & \multicolumn{1}{c|}{$\begin{array}{c}\text { Contrast Ratio } \\
\kappa=\frac{\sigma_{3}}{\sigma_{2}}\end{array}$} \\
\hline 7 & $\begin{array}{l}2.8,4.3,5.8, \text { and } \\
7.4\end{array}$ & 1.9 & $\begin{array}{l}1.4,2.7,4.9 \text {, and } \\
11.7\end{array}$ \\
\hline 8 & 2.8 & $\begin{array}{l}1.0,1.3,1.6, \text { and } \\
1.9\end{array}$ & $\begin{array}{l}1.4,2.8,4.9 \text {, and } \\
11.7\end{array}$ \\
\hline
\end{tabular}

\subsection{Numerical Methods}

The Electrical Property Enhanced Tomography method predicts boundary current patterns and internal electrical properties using numerical methods. Experiments will be performed that compare two numerical methods, the charge-charge correlation method (CCCM) derived from the Green's theorem and the indirect reconstruction method (IRM) using finite difference methods and Newton-Raphson methods. The section describes how each method is applied.

\subsubsection{Application of Charge-charge Correlation Method}

Equations (3-31), (3-43), and (3-45) in Chapter 3 are tailored for the two- and threemedia experiments previously described. Figure 4-3 represents a top view schematic of the domain with a circular shaped base of medium 2 (for the two media case). The domain is square with a side length of $23.2 \mathrm{~cm}$ (i.e. $\mathrm{a}=\mathrm{b}=23.2 \mathrm{~cm}$ in equation (3-31)). The center of mass of medium 2 is located at coordinates $\left(\mathrm{x}_{2}, \mathrm{y}_{2}\right)$ while the charge $\delta \mathrm{q}_{\mathrm{i}}$ is located at coordinates $\left(\mathrm{x}^{\prime}{ }_{\mathrm{i}}, \mathrm{y}^{\prime}{ }_{\mathrm{i}}\right)$. 
The angle $\theta_{\mathrm{l}}$ is measured with respect to the positive y axis (3 o'clock position on the sample). Coordinates $\mathrm{x}_{\mathrm{i}}$ and $\mathrm{y}_{\mathrm{i}}$ are rewritten in terms of $\mathrm{x}_{2}, \mathrm{y}_{2}$, and $\mathrm{d}_{2}$, and the angle $\theta_{\mathrm{i}}$ (equations (4-1) and (4-2)) and substituted into equation (3-31) of the previous chapter to express the coefficient $\mathrm{B}_{\mathrm{i}}(\mathrm{l})$ in these terms (equation (4-3)).

$$
\begin{gathered}
x_{i}^{\prime}=x_{2}+\frac{d_{2}}{2} \cdot \cos \left(\theta_{i}^{\prime}\right) \\
y_{i}^{\prime}=y_{2}+\frac{d_{2}}{2} \cdot \sin \left(\theta_{i}^{\prime}\right) \\
\left.B_{l}(l)=\sin \left(\frac{l \pi\left(x_{c}+\frac{d_{2}}{2} \cos \left(\theta_{i}^{\prime}\right)\right)}{23.2}\right) \frac{\sinh \left(l \pi\left(1-\frac{\left(y_{2}+\frac{d_{2}}{2} \sin \left(\theta_{i}^{\prime}\right)\right)}{23.2}\right)\right)}{\sinh (l \pi)}\right)
\end{gathered}
$$




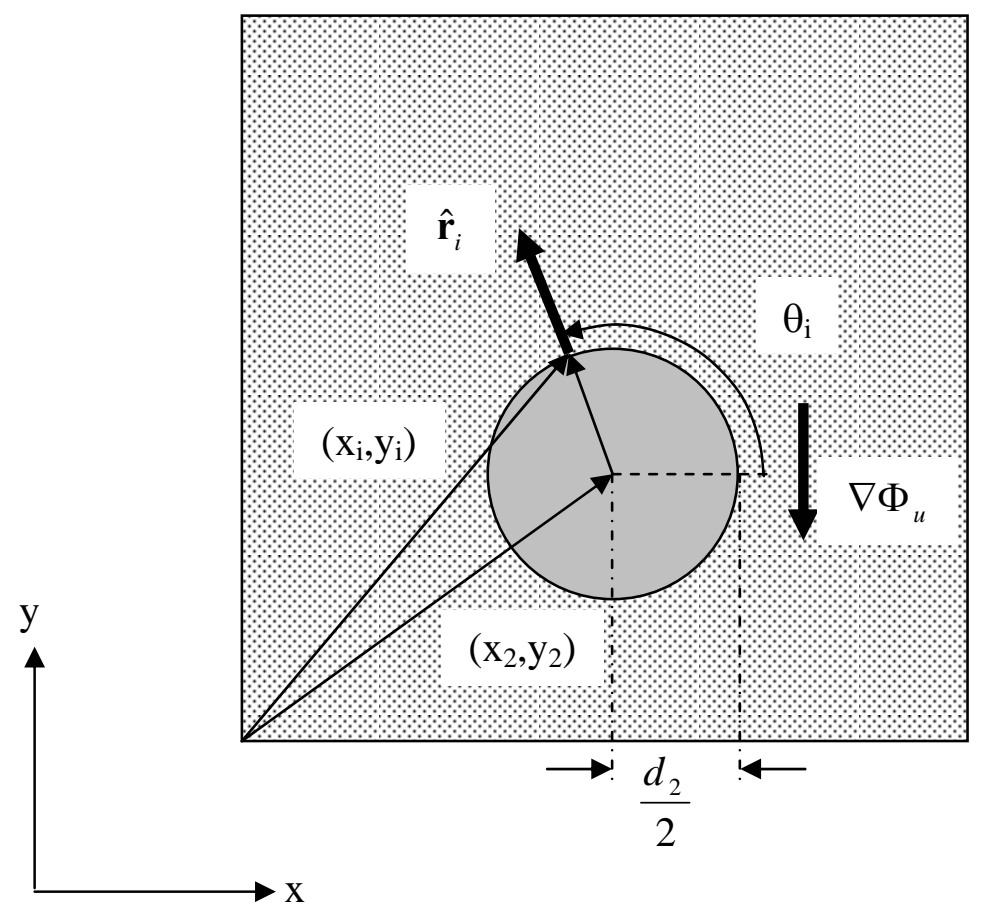

Figure 4-3: The two media case with circular shaped $2^{\text {nd }}$ medium The coordinate transformation for a circular Medium 2 is illustrated. The point along the boundary, $\mathrm{x}_{\mathrm{i}}$ and $\mathrm{y}_{\mathrm{i}}$, will be expressed in terms of the center of mass, $\mathrm{x}_{2}$ and $\mathrm{y}_{2}$, diameter, $\mathrm{d}_{2}$, and angle $\theta_{\mathrm{i}}$ measured with respect to the x-axis.

The dot product in equation (3-43) is computed by substituting $\hat{\mathbf{r}}_{i}$ (replacing the vector $\hat{\mathbf{n}}_{i}$ ), defined in equation (4-4), and $\nabla \Phi$, defined in equation (4-5), to produce equation (4-6). Additionally, the elemental area, Sarea, in the same equation is computed from the product of the height and arc-length (equation (4-7)). This dot product and elemental area are substituted back into equation (3-43) to produce equation (4-8)).

$$
\begin{gathered}
\hat{\mathbf{r}}_{i}=\cos \left(\theta_{i}^{\prime}\right) \hat{\mathbf{x}}+\sin \left(\theta_{i}^{\prime}\right) \hat{\mathbf{y}} \\
\left.\nabla \Phi_{u}\right|_{\left(x_{i}, y_{i}\right)}=\left\|\left.\nabla \Phi\right|_{\left(x_{i}, y_{i}\right)}\right\|\left(\cos \left(\lambda_{i}\right) \hat{\mathbf{x}}+\sin \left(\lambda_{i}\right) \hat{\mathbf{y}}\right) \\
\left.\hat{\mathbf{r}}_{i} \cdot \nabla \Phi_{u}\right|_{\left(x_{i}, y_{i}\right)}=\left\|\left.\nabla \Phi_{u}\right|_{\left(x_{i}, y_{i}\right)}\right\| \cos \left(\theta_{i}^{\prime}+\lambda_{i}\right) \\
\text { darea }_{i}=c \cdot\left(\frac{d_{2}}{2}\right) \cdot \delta \theta
\end{gathered}
$$




$$
\mathrm{A}_{l}^{\prime}=-\sigma_{1}\left(\frac{\delta x \cdot c}{a}\right) \cdot \sum_{i=1}^{\mathrm{N}} \mathrm{B}_{i}(l) \cdot\left(\left\|\left.\nabla \Phi_{u}\right|_{\left(x_{i}, y_{i}\right)}\right\| \cdot \cos \left(\theta_{i}+\lambda_{i}\right) \cdot\left(\frac{d_{2} \delta \theta}{2}\right)\right)
$$

The current, I' ${ }^{\mathbf{p}}$, in equation (3-45) is computed using $\mathrm{A}^{\prime}{ }_{1}$ and $\mathrm{B}_{\mathrm{i}}$ from equations (4-3) and (4-8). The relationship between the measured and predicted current patterns remains unchanged. For the purpose of clarity, the relationship for a single medium is restated in equation (4-9) and the computation of the mediums electrical properties is restated in equation (4-10).

$$
\begin{gathered}
\mathbf{I}^{m}=\xi \cdot \mathbf{I}^{p}\left(x_{2}, y_{2}, d_{2}\right) \\
\kappa_{21}=\frac{1+\xi}{1-\xi}
\end{gathered}
$$

For experiments using three media, the measured current pattern is related to the weighted sum of both predicted currents $\left(\mathbf{I}^{\prime} \mathrm{P}\left(\mathrm{x}_{2}, \mathrm{y}_{2}, \mathrm{~d}_{2}\right)\right.$ and $\left.\mathbf{I}^{\mathrm{P}}\left(\mathrm{x}_{3}, \mathrm{y}_{3}, \mathrm{~d}_{3}\right)\right)$. The relationship is expressed in matrix form (equation (4-11)). The variables $\xi_{1}$ and $\xi_{2}$ are determined by regressive means and used to compute the electrical properties $\kappa_{21}$ and $\kappa_{32}$ (equations (4-12) and (4-13)).

$$
\begin{gathered}
\mathbf{I}^{m}=\left[\mathbf{I}^{\prime p}\left(x_{2}, y_{2}, d_{2}\right), \quad \mathbf{I}^{p}\left(x_{3}, y_{3}, d_{3}\right)\right] \cdot\left(\begin{array}{l}
\xi_{1} \\
\xi_{2}
\end{array}\right) \\
\kappa_{21}=\frac{1+\xi_{1}}{1-\xi_{1}} \\
\kappa_{32}=\frac{1+\xi_{2}}{1-\xi_{2}}
\end{gathered}
$$

\subsubsection{Application of the Indirect Reconstruction Method}

The indirect reconstruction method (IRM) is divided into two steps, the forward solution and the inverse solution, which are performed sequentially and repeatedly for a predetermined number of iterations. The forward solution is performed using the Finite Difference Method (FDM) while the inversion solution is performed using the Newton-Raphson (N-R) method.

To apply the FDM, the domain is first discretized. Figure 4-4 illustrates a domain with three media. Figure 4-5 illustrates a discretized version of the same domain. The discretized domain is converted into a map of the electrical properties, where each element of the map is assigned an electrical property value and every vertex represents a potential point. Figure 4-6 illustrates the domain map. Each shade of the map represents a unique electrical property. This 
figure is represented by three different electrical properties. Figure 4-7 illustrates a close-up of four elements and 5 vertices. The four elements meet at a common point (one of the five vertices) and the remaining four vertices are located at points where two of the elements touch.

The forward solution first computes the internal potential field $\Phi$ using the Jacobi’s method [149]. The Jacobi's method solves equation (4-14) by computing the potential at a given discretized point. The method averages surrounding discretized potentials weighted by the surrounding electrical properties. The technique is guaranteed to converge to a solution. The Jacobi method is iterative and terminates when the residual (equation (4-15)) falls below a preset threshold (which is chosen to be $10^{-11}$ ). The resulting potential field is a 93 by 93 array (columns by rows) of complex numbers.

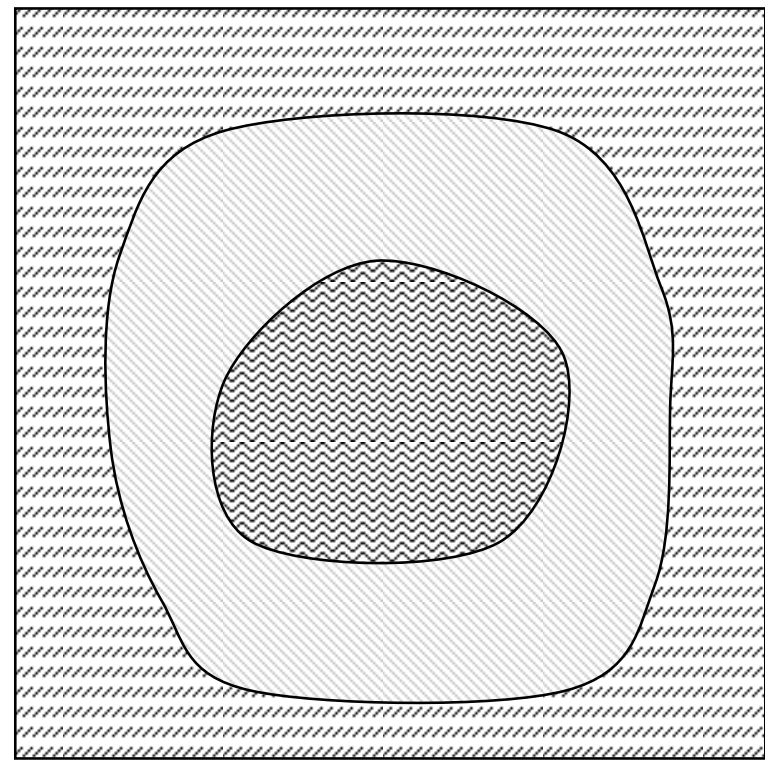

Figure 4-4: Domain with 3 media

A three-media, square domain is illustrasted.

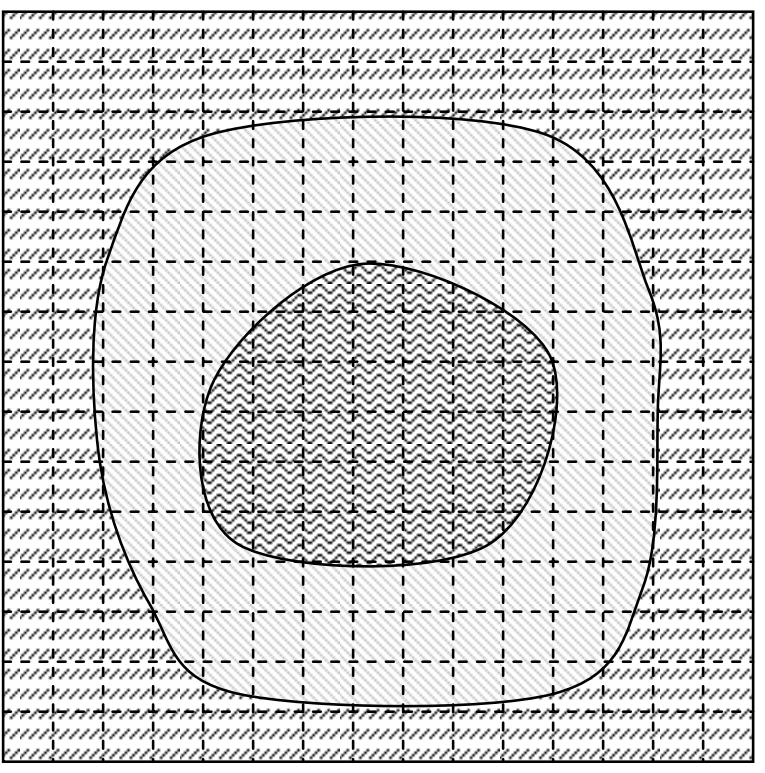

Figure 4-5: Discretized Domain

The three-media domain is discretized. 


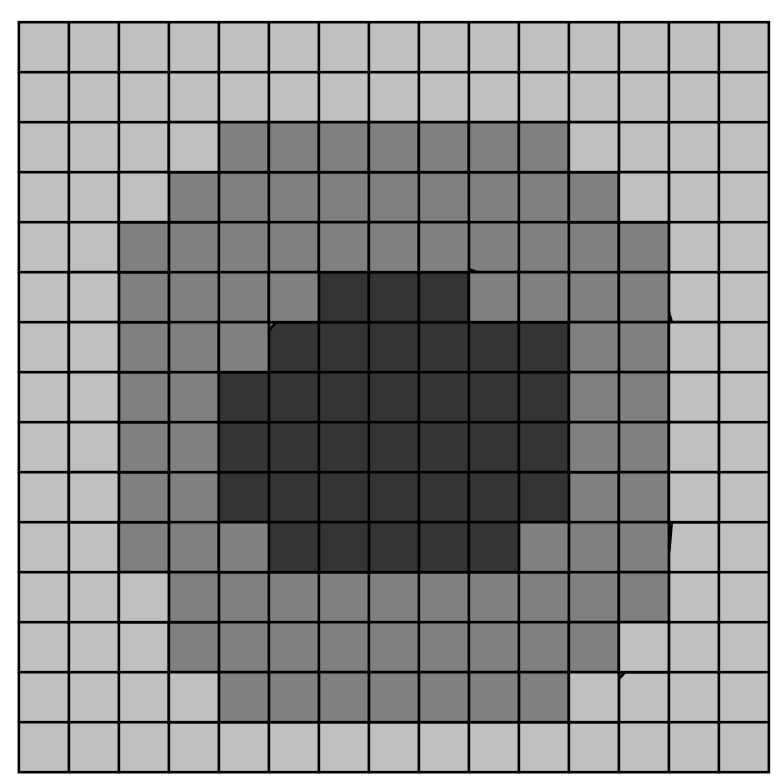

Figure 4-6: Domain Map

The discretize domain is replaced with a domain map, where each element in the map represents one of the discrete elements from the domain.

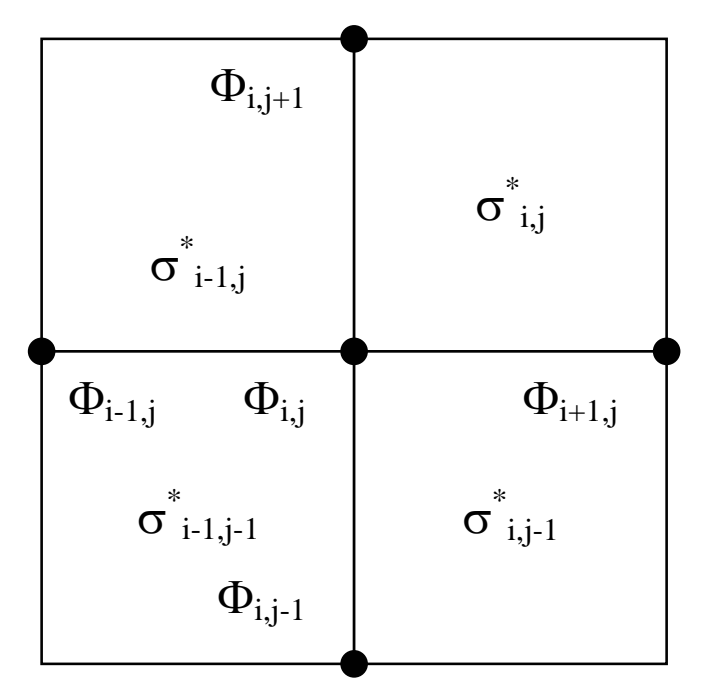

Figure 4-7: Close-up view of potential vertices and electrical property elements

Finite difference methods use the domain map to compute potentials throughout the domain. The potentials are computed at each vertex.

$$
\begin{aligned}
& \Phi_{i, j}^{n+1}= \\
& \frac{\left(\sigma_{i, j-1}^{n}+\sigma_{i, j}^{n}\right) \Phi_{i+1, j}^{n}+\left(\sigma_{i-1, j}^{n}+\sigma_{i, j}^{n}\right) \Phi_{i, j+1}^{n}+\left(\sigma_{i-1, j}^{n}+\sigma_{i-1, j-1}^{n}\right) \Phi_{i-1, j}^{n}+\left(\sigma_{i, j-1}^{n}+\sigma_{i-1, j-1}^{n}\right) \Phi_{i, j-1}^{n}}{2\left(\sigma_{i, j-1}^{n}+\sigma_{i, j}^{n}+\sigma_{i-1, j-1}^{n}+\sigma_{i-1, j}^{n}\right)}
\end{aligned}
$$

where $i$ is the column

$\mathrm{j}$ is the row

$\mathrm{n}$ is the iteration count

where $\mathrm{M}$ and $\mathrm{N}$ are 93

$$
\text { Residual }=\frac{\sum_{i=1}^{M} \sum_{j=1}^{N}\left\|\Phi_{i, j}^{n+1}-\Phi_{i, j}^{n}\right\|}{M N}
$$

The FDM is performed for the uniform and non-uniform cases producing two internal potential fields, $\Phi_{\mathrm{u}}$ and $\Phi_{\mathrm{p}}$, respectively. From these cases the difference potential field, $\Delta \Phi=\Phi_{\mathrm{p}}-\Phi_{\mathrm{u}}$, is computed

The current at each electrode, $\mathrm{k}$, is computed from the current density, $\mathbf{J}$, passing through the electrode cross-sectional area, AREA (equation (4-16)). The gradient is computed by the difference method (equation (4-17)). Here $\delta y$ is the distance in the $y$-direction between adjacent vertices. 


$$
\begin{gathered}
I^{p}\left(x_{k}, 0\right)=-\left.\sigma_{1}^{*} \cdot \nabla \Delta \Phi\right|_{\left(x_{k}, 0\right)} \cdot \operatorname{AREA} \\
\left.\nabla \Delta \Phi\right|_{\left(x_{k}, 0\right)}=\frac{\Delta \Phi_{k, 1}-\Delta \Phi_{k, 0}}{\delta y}
\end{gathered}
$$

The inverse processing applies the Newton-Raphson (N-R) method (equation (4-18)) to compute an update (or correction) to the electrical properties $\left(\sigma^{*}\right)$. The measured and predicted current patterns are represented as column vectors, as defined in equation (3-46 ). The predicted current, $\mathbf{I}^{\mathrm{P}}$, is a function of the electrical properties for all three media (equation (4-19)). The correction to the electrical properties, $\Delta \boldsymbol{\sigma}$, is represented by a column vector with 6 elements (equation (4-20)). The derivative matrix (equation (4-21)) is represented as a 6 by 15 (column by row) matrix, where each column is computed by difference equations (equation (4-22)).

$$
\begin{gathered}
\mathbf{I}^{m}=\mathbf{I}^{p}+\frac{\partial \mathbf{I}^{p}}{\partial \sigma^{*}} \Delta \boldsymbol{\sigma}^{*} \\
\mathbf{I}^{p}\left(\sigma^{*}\right)=\mathbf{I}^{p}\left(\sigma_{1}, \sigma_{2}, \sigma_{3}, \varepsilon_{r 1}, \varepsilon_{r 2}, \varepsilon_{r 3}\right) \\
\Delta \boldsymbol{\sigma}=\left(\Delta \sigma_{1}, \quad \Delta \sigma_{2}, \quad \Delta \sigma_{3}, \quad \Delta \varepsilon_{r 1}, \quad \Delta \varepsilon_{r 2}, \quad \Delta \varepsilon_{r 3},\right)^{\mathrm{T}} \\
\frac{\partial \mathbf{I}^{p}}{\partial \sigma^{*}}=\left(\frac{\partial \mathbf{I}^{p}}{\partial \sigma_{1}}, \quad \frac{\partial \mathbf{I}^{p}}{\partial \sigma_{2}}, \quad \frac{\partial \mathbf{I}^{p}}{\partial \sigma_{3}}, \quad \frac{\partial \mathbf{I}^{p}}{\partial \varepsilon_{r 1}}, \frac{\partial \mathbf{I}^{p}}{\partial \varepsilon_{r 2}}, \quad \frac{\partial \mathbf{I}^{p}}{\partial \varepsilon_{r 3}}\right) \\
\frac{\partial \mathbf{I}^{p}}{\partial \sigma_{1}}=\frac{\mathbf{I}^{p}\left(\sigma_{1}+\delta\right)-\mathbf{I}^{p}\left(\sigma_{1}\right)}{\delta}
\end{gathered}
$$

The correction to the electrical properties, $\Delta \sigma^{*}$, is computed by performing a matrix multiplication using the inverse of equation (4-23)). The derivative matrix is not square. The singular value decomposition (SVD) method is used to compute the inverse since it is a very powerful and stable tool for the solution of linear systems [149].

$$
\Delta \boldsymbol{\sigma}^{*}=\left[\frac{\partial \mathbf{I}^{p}}{\partial \sigma^{*}}\right]^{-1} \cdot\left(\mathbf{I}^{m}-\mathbf{I}^{p}\right)
$$

The electrical properties are updated (equation (4-24)) by adding the change in electrical properties to the current estimate for the electrical properties. At each correction the iteration index, $\mathrm{k}$, is incremented (equation (4-25)). The indirect method ends when the iteration count, $\mathrm{k}$, is equal to the predetermined maximum iteration maximum number of iterations, which is 
chosen to be 5 iterations. There was typically no change in the predicted electrical properties after 5 iterations.

$$
\begin{gathered}
\sigma^{k+1}=\sigma^{k}+\Delta \sigma \\
k=k+1
\end{gathered}
$$

\subsection{Sample Preparation}

Four types of sample medias are used in the experiments; saline solution, agar, liver, and oatmeal. The preparation and storage of agar, liver and oatmeal is described below.

\subsubsection{Agar}

Agar is a gelatinous colloidal extractive of a red alga genera Gelidium, Gracilaria, and Eucheuma [150]. It is sold in powder form. Agar is prepared by mixing one liter of a saline solution of known conductivity with $40 \mathrm{~g}$ of agar power. The mixture is heated and allowed to come to a boil. It thickens as it is heated, thus it must be continually stirred. The hot agar is poured into molds and allowed to cool. The now solid agar is refrigerated when not in use. It is stored in airtight plastic bags filled with saline solution with the same conductivity as when it is originally prepared. Samples are prepared in 1.0, 1.3, 1.6, 1.9 and $20.3 \mathrm{~cm}$ molds.

\subsubsection{Liver}

Freshly slaughtered calves liver was purchased within 2 hours of slaughter. The liver weighed between 4.5 to $5.5 \mathrm{Kg}$ and at the time of purchase the liver was warm to the touch. The liver was cut into a circular disk about $20.3 \mathrm{~cm}$ in diameter using a 'cookie cutter' template. It was cut to $3 \mathrm{~cm}$ thickness using a butcher knife. A hole was cut into the liver for the placement

of medium 3, a mixture of oatmeal, salt and water discussed below. The hole was cut with a 1.3 cm diameter hole punch. Liver was used for one day, and then discarded.

\subsubsection{Oatmeal}

The oatmeal mixture is prepared from 4.5 liters of dry oatmeal, 2 liters of distilled water, and 9 grams of salt. The oatmeal mixture is divided into smaller volumes, placed in plastic zip- 
lock bags and stored in a freezer. On the day of an experiment the oatmeal is removed from the freezer and allowed to warm to room temperature.

\subsection{Determining Internal Boundary Locations a priori}

The EPET method uses a priori positional information to compute the electrical properties. EPET does not attempt to create the image with the electrical data but rather adds electrical property information to the existing modality and, in fact, requires the data from the other modality to locate the position of internal structures in the object. Experiments with agar media used Plexiglas templates with pre-drilled holes to precisely determine size and position of the medium. Experiments using liver and oatmeal used a second imaging modality (digital photos) to determine the boundary positions.

\subsubsection{Templates for Two Media Experiments}

Plexiglas templates ( $23 \mathrm{~cm}$ by $23 \mathrm{~cm}$ by $0.5 \mathrm{~cm}$ ) were used to perform all the two media experiments. The plastic templates have pre-drilled slots located at positions described in the first section of this chapter. Separate templates are used for different slot diameters. Slots are precisely machined to allow less than $1 \mathrm{~mm}$ of play in the sample placement. The template sits on a Plexiglas lip about $1 \mathrm{~cm}$ above the bath water surface (approximately $2 \mathrm{~cm}$ above the sample holder floor). The agar medium is inserted through the slot in the template and rests on the sample holder floor.

\subsubsection{Position Spacers for Three Media Experiments with Agar}

The positions of the 'holes' in the large agar disk are well defined within the large agar disk. Consequently, the effort is limited to determining the position of the large disk $\left(\mathrm{x}_{1}\right.$ and $\left.\mathrm{y}_{1}\right)$. Four $1.3 \mathrm{~cm}$ diameter spacer rods are temporarily placed in the tank, one along each wall of the tank. The large agar disk, which is firm to the touch, is placed in the tank so that if fits snuggly between the four rods. The large agar disk is rotated until the appropriate 'hole' (i.e. the hole appropriate for the experiment) is properly aligned. The rods are then removed from the tank. The center of the agar disk is positioned at $\mathrm{x}_{1}=11.6 \mathrm{~cm}$ and $\mathrm{y}_{1}=11.3 \mathrm{~cm}$. 


\subsubsection{Digital Images for Three Media Experiments with Liver and Oatmeal}

A digital image is used to represent an axial slice from some imaging technique (e.g. CT) that would be available when applying this technique in a clinical setting. The process for generating a 2D model from a digital camera photo is completed in five steps:

1. Image acquisition

2. Image correction

3. Image discretization

4. Boundary identification

5. Filling elements between boundaries

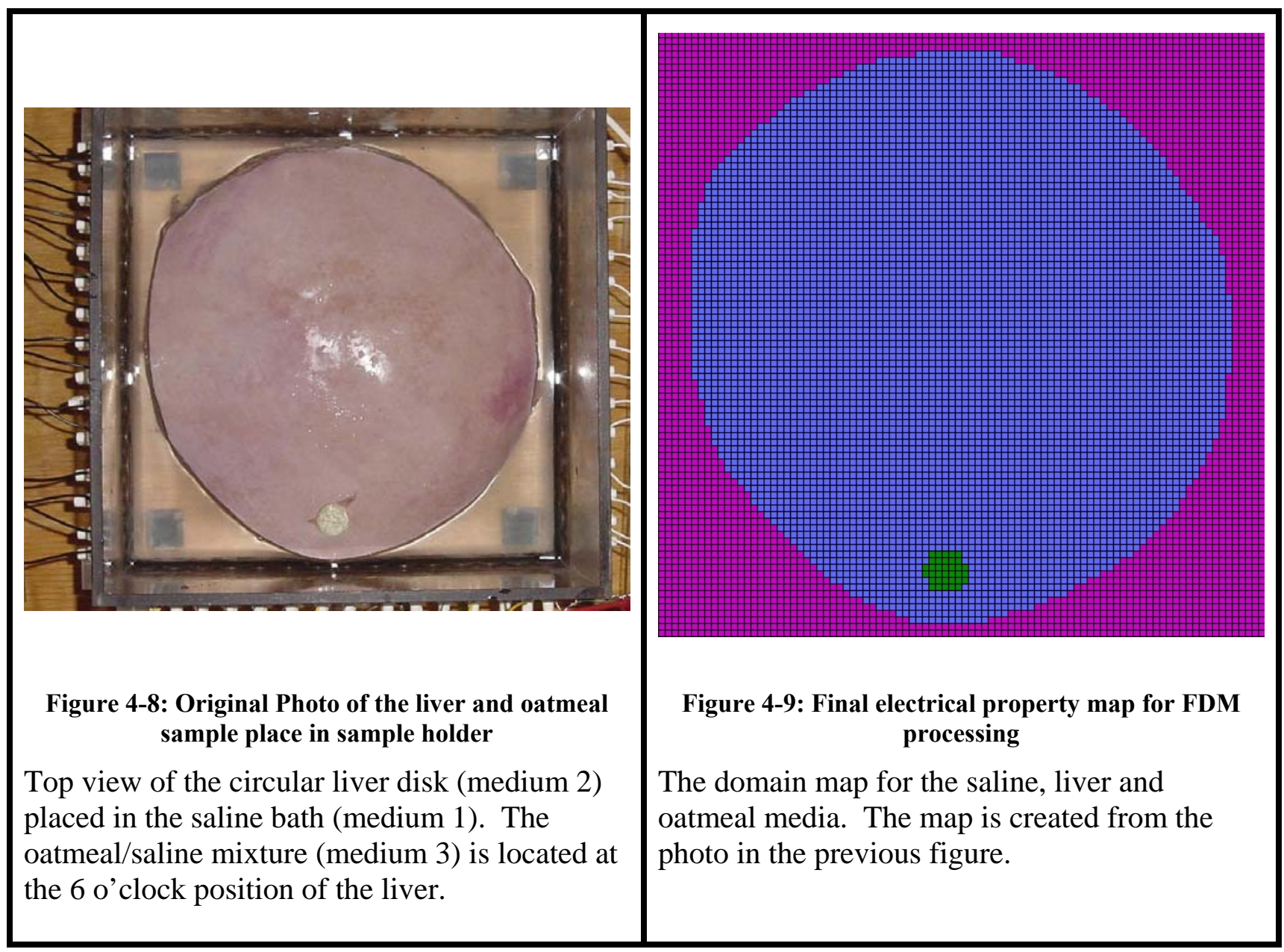

A digital photo is initially acquired using a digital camera manually held above the sample holder (Figure 4-8). The image is rotated, stretched, and cropped to fit a square array template. It is then discretized, divided into $\mathrm{N}$ by $\mathrm{N}$ square shaped elements (dashed lines delineate the boundaries) (symbolically illustrated in Figure 4-5). Each element represents the electrical properties for the area occupied by the discretized element. The medium type at boundaries between different media is assigned to the medium that occupies more than $50 \%$ of 
the area of that discretized element. Finally, all elements within the bounds are assigned with identical elements (Figure 4-9). The initial photo (Figure 4-8) and final ‘map’ (Figure 4-9) are illustrated below.

\subsection{Experimental Procedures}

\subsubsection{Two Media Experiments}

The sample holder is filled with the salt-water solution of known conductivity (medium 1). The temperature of medium 1 is measured with a thermocouple and recorded. The position template is placed in tank (above the water line). The measurement of the current proceeds in the following eight steps:

1. Current is measured at electrodes 1 through 15 with only medium 1 in the sample holder. Measurement of all 15 electrodes is called a 'pass'.

2. Medium 2 is placed through the template slot until it rests on the on the sample holder floor.

3. Current is measured at electrodes 15 through 1.

4. Medium 2 is removed.

5. Repeat steps 1 to 4 five times.

6. One final measurement is collected at electrodes 1 through 15 with only medium 1 in the sample holder.

7. The template is removed.

8. Finally, the temperature of medium 1 and 2 is measured and recorded.

\subsubsection{Three Media Experiments with Agar}

The sample holder is filled with the salt-water solution (medium 1). The temperature of medium 1 is measured with a thermocouple and recorded. The current is measured for 10 passes (i.e. from electrodes 1 to 15, then 15 to 1, and so forth five times). Medium 2 (large agar disk) is placed and positioned in the tank. Displaced water exits the tank through an overflow valve and

collects in a reservoir. The agar disk is NOT covered with water. All 'holes' in the agar disk are initially filled with agar plugs (medium 3) with the same conductivity as the agar disk (medium 2) technically making it a two-medium case. The measurement of the current proceeds in the following steps:

1. Current is measured at electrodes 1 through 15.

2. Medium3 is exchanged for an agar rod with the test conductivity.

3. Current is measured at electrodes 15 through 1.

4. Medium 3 is replaced with the agar rod with the same conductivity as medium 2. 
5. Repeat steps 1 to 4 ten times (10 passes).

6. Make one final measurement at electrodes 1 through 15 (matching agar is present)

7. Medium 2 is removed.

8. Medium 1 in the reservoir is returned to the tank.

9. Finally, the current was measured for 10 passes.

\subsubsection{Three Media Experiments with Liver and Oatmeal}

The sample holder is filled with the saline solution (medium 1). The current is measured for 5 passes. Medium 1 conductivity is measured using a handheld conductivity meter. Saline bath and liver (media 1 and 2) temperatures are measured using a digital thermometer. The liver with oatmeal plug (media 2 and 3) is placed in the tank. Displaced water rises and exits the tank through an overflow valve where it is collected in a reservoir. The liver is NOT covered with water. Medium 1 conductivity is measured a second time. The digital image of the liver in the tank is acquired. The current is measured for 10 passes. Medium 1 conductivity is measured a third time. Media 2 and 3 are removed from the tank and water in the reservoir is poured back into the tank. Medium 1 conductivity is measured for a fourth and last time. Finally, the current is measured for 5 passes after removing the liver.

\subsection{Experimental Set-up}

The data acquisition system has 7 functional units: an excitation source, a signal driver, a sample holder, output signal sensor, a signal demodulator and a computer for system control and data storage. The excitation source produces a voltage signal with known amplitude, phase, and frequency. This signal is input to a buffer circuit, the voltage driver, which drives the electrodes on the sample holder. The sample holder functions as the interface between the driver/sensor electronics, a rigid framework for the electrodes, and a water-tight container. The output signal, a current, is sensed and demodulated to produce a digital signal (magnitude and phase). Finally, a personal computer controls the excitation source and demodulator instrumentation and stores the acquired data.

The experimental set-up is sketched in Figure 4-10. Item 1 is a Gateway 2000 Pentium $133 \mathrm{MHz}$ with a GPIB card installed to provide two-way communication with item 2, the Solartron Instruments 1260 (SI 1260) Impedance Phase/Gain Analyzer [151]. The computer operates ZPLOT software, produced by Scribner Associates [152], to control the SI 1260, a dualpurpose instrument that provides both an output voltage source (excitation) signal and measures 
a sensed signal. ZPLOT controls the excitation signal magnitude and frequency, specifies the measurement mode, and controls the amount of acquired data. The Solartron 1260 generates a $1.0 \mathrm{~V}_{\text {rms }}$ excitation signal at $50 \mathrm{kHz}$ and measures a voltage difference. The sensed signal is acquired for at least 0.2 seconds and produces a demodulated measurement of the average difference voltage, $\mathrm{V}_{2}-\mathrm{V}_{1}$ across a sense resistor. The measured data are stored in text files on the personal computer's hard disk drive. 


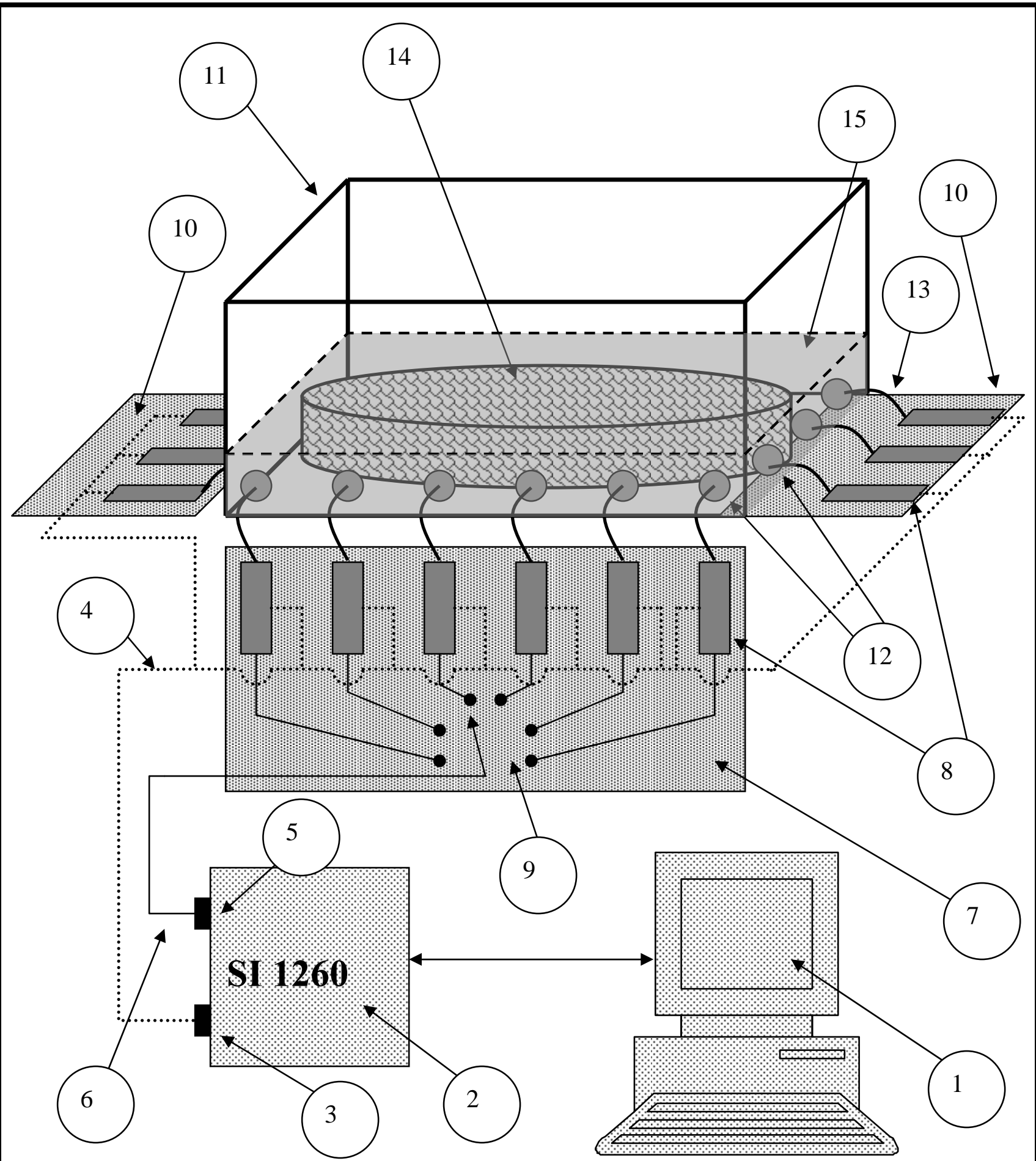

Figure 4-10: Overview of the implemented data acquisition system

Overview of the complete data acquisition system. The system is composed of: (1) computer processor with data storage device, (2) Solartron 1260 Impedance Phase/Gain Analyzer, (3) Excitation signal port, (4) source signal cable, (5) sensed signal port, (6) sense signal cable, (7) driver/sensor board, (8) driver/sensor electronics, (9) signal switch, (10) side gradient driver, (11) data acquisition tank, (12) electrode, (13) connection to electrode, (14) medium 2, and (15) saline. 
The SI 1260 has three ports, one output port (Item 3) and two input ports (Item 5). The input ports measures voltage drop $\left(\mathrm{V}_{2}-\mathrm{V}_{1}\right)$, across a sense resistor (Item 8), from which the 'sensed' current is computed. Oscilloscope probes connect the excitation and measured signals from the SI 1260 to the Main Driver/Sensor and Gradient Driver (Items 7 and 10, respectively) electronics boards. The paths of the excitation (Item 4) and measured signals (Item 6) are presented as dotted and solid black lines respectively. These pathways originate/terminate at the output/input ports of the SI 1260 and terminate/originate at the driver/sensor circuitry located on the main and gradient driver electronics boards.

The voltage driving and current sensing functions are performed by the dual function driver/sensor circuitry (Item 8) located on the main and gradient driver/sensor electronics boards. The driver electronics delivers an applied voltage signal to the electrodes (Item 12) of the sample holder (Item 11). This applied signal interacts with the contents of the sample holder resulting in the flow of electric current. The sensor function of the electronics senses the current flow passing through the electrodes.

The voltage driver and current sensing element (Item 8 in Figure 4-10) are combined within the same circuit. The basic circuit design is adapted from Blad [153], Blad et al [154] and Hartov et al [155] and is presented in Figure 4-11. An operational amplifier (Analog Devices AD828) is configured in an inverting mode with a gain of -1 . Resistors $R_{1}, R_{2}$, and $R_{3}$ have a resistance of $10 \mathrm{k} \Omega$ and are connected in a typical inverting mode configuration. The precision resistor $R_{\mathrm{s}}(1 \mathrm{~K} \Omega)$ functions as the current sensing element used to measure the current flowing through the electrode. The current that passes through a given electrode is computed using Ohm's law (equation (4-26)) from the voltage drop, $V_{2}-V_{1}$, across $R_{s}$.

$$
I=\frac{\left(V_{2}-V_{1}\right)}{R_{s}}-\frac{V_{1}}{R_{2}}
$$

Item 11, the sample holder, is a fixture that holds the media. The sample holder geometry is very important because it establishes the conditions for the Green's Function used. The sample holder holds the saline bath, medium 1(Item 15), and the other media being tested (Item 14). A more detailed illustration of the sample holder is presented in Figure 4-12.

The sample holder is a square shaped tank fabricated from transparent acrylic. It is lined with 60 stainless steel electrodes, 15 electrodes along each side of the sample holder tank, (Item 12). Each electrode has a $12.7 \mathrm{~mm}$ diameter with a separation gap of $2.54 \mathrm{~mm}$ between the electrodes. The overall size of the sample holder is $23.1 \mathrm{~cm}$ along each side. The liquid level is 
regulated by a drain spout mounted to the sample holder wall $1.88 \mathrm{~cm}$ above the sample holder floor. The liquid level is the same distance above the electrodes as below.

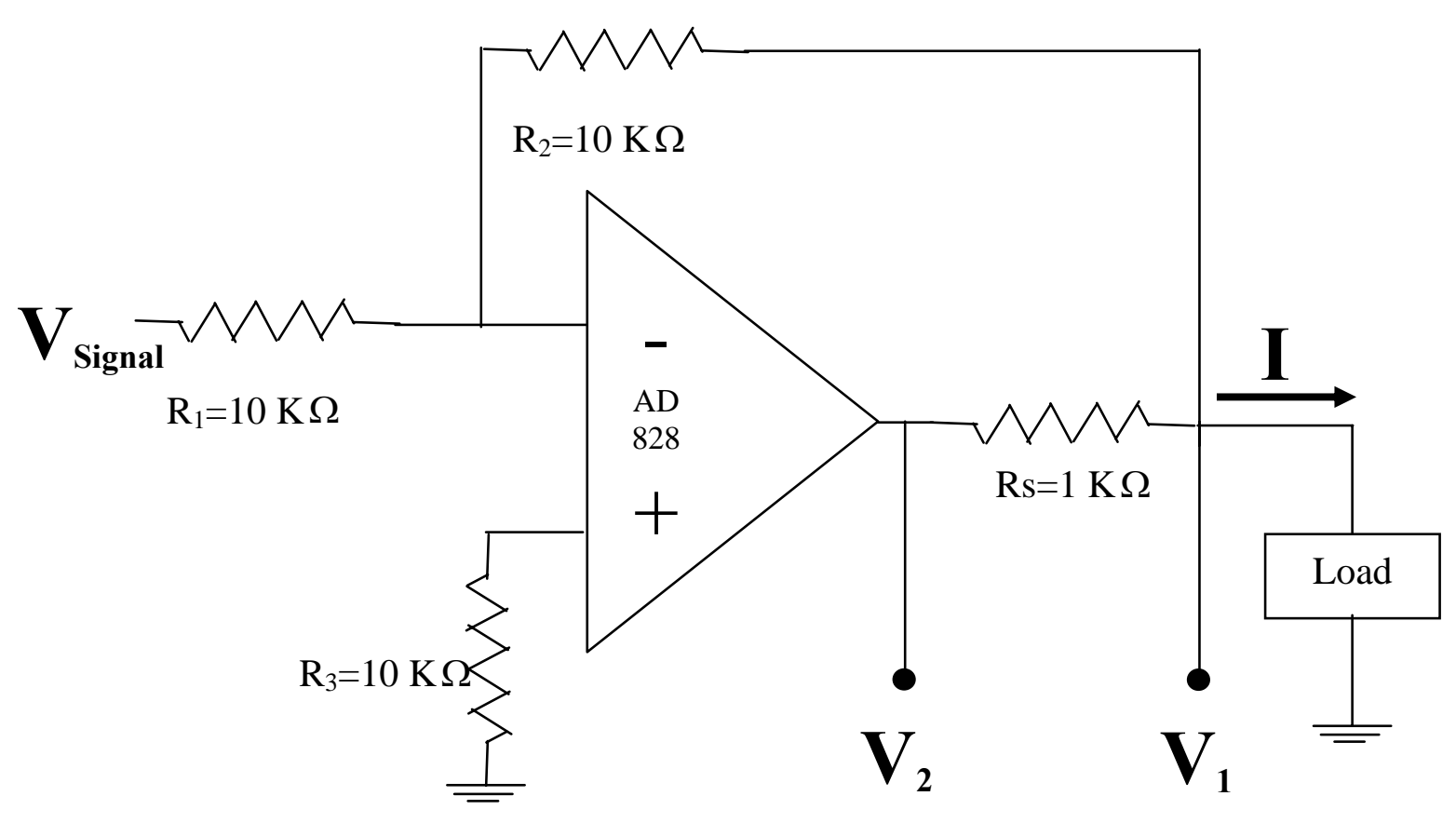

Figure 4-11: Driver/Sensor Circuit

The driver/sensor circuit is adapted from $[153,154,155]$. The driving signal, $\mathrm{V}_{\text {signal, }}$ is magnified with gain -1 and supplies current to the load. The current is computed from the measured potential difference, $V_{2}-V_{1}$, across the sense resistor $R_{s}$.

The applied voltage along the boundary of the sample holder is designed to produce a current flow primarily along the $+y$-direction. The square-shaped sample holder lends itself to the implementation of a simple potential pattern to produce an electric field, and consequently a current flow, along the y-direction. A potential pattern of constant phase, but different magnitude is applied to all electrodes along the near and far sides (defined in Figure 4-12), while simultaneously applying a potential with a gradient along the left and right sides. The near side voltage was maintained at 1 Vrms while the far side was held at ground. The voltage along the left and right side decreased linearly with increasing distance (from near to far sides) from 1 Vrms to ground $\left(V=\frac{-1}{23.2}+1[\right.$ Vrms $\left.]\right)$ in 15 steps.

Each electrode in the tank is assigned a number. Electrode numbers range from 1 to 15 along the near side (from left to right). The numbering continues along the right side from 16 to 
30 (near to far), from 31 to 45 along the far side (right to left), and from 46 to 60 along the left side (far to near).

Digital imagery of the test media is used in the implementation of the EPET theory. The DSC-S50 digital camera manufactured by SONY ${ }^{\mathrm{i}}$ is used for this purpose. The camera produces color images with a 1600x1200 pixel resolution. Images are captured and stored on a memory stick and later transferred to the Gateway 2000 in JPEG format. The operator holds the camera $86 \mathrm{~cm}$ directly above the surface of the sample holder floor. The camera pointed downward perpendicular to the sample in the sample holder.

The saline bath is a controlled element in the experiment. It is easily retrievable and thus measurement of its electrical properties during the course of the experiment does not violate the notion of identifying the electrical properties of the unknown object. Furthermore, its acquisition does not alter results since sampled saline is returned to the experimental sample holder.

During the experiment, the temperature is monitored in media 1, 2 and 3 because electrical properties of both biological and non-biological media are temperature dependent. These media are all at ambient temperature. In the event measured temperature varied dramatically, temperature data will be used to account for some of the variations in the computed electrical property values. Temperature data are acquired using a handheld digital stem thermometer manufactured by Tenma ${ }^{\text {ii }}$ and a HH82 thermocouple manufactured by Omega ${ }^{\text {iii }}$. A sample of the saline is removed from the sample holder using a syringe and injected into a clean container. The probe from the Tenma stem thermometer is inserted into the container and the temperature is measured. Once the measurement is complete, the saline is returned to the sample holder.

\footnotetext{
${ }^{i}$ Sony Electronics Inc., 1 Sony Drive Park, Ridge, NJ 07656, Manual Number: 3-060-523-12(1)

ii Digital Stem Thermometer, Tenma, 72-6789, http://www.tenma.com/

iii OMEGA Engineering, INC., One Omega Drive, Stamford, Connecticut, 06907-0047
} 

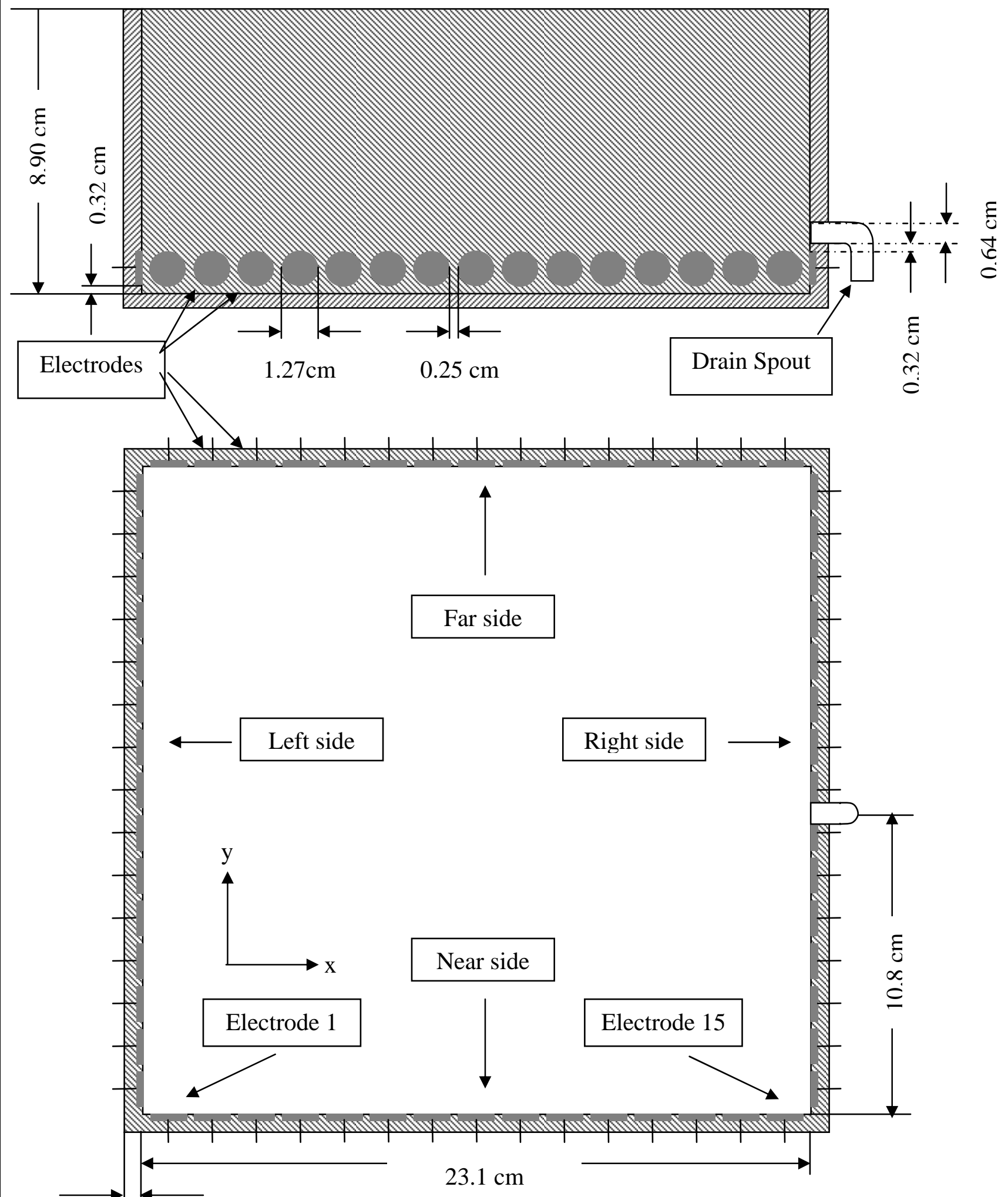

$0.64 \mathrm{~cm} \quad$ 12: (Above) Side view and (Below) top view of the data acquisition tank

Top and side views of the data acquisition tank are presented. The tank dimensions and electrode position (horizontal and vertical) are clearly illustrated. 


\section{RESULTS AND DISCUSSION}

\subsection{Raw Experimental Data: Description and Characterization}

\subsubsection{Typical Raw Current Pattern}

Figure 5-1 illustrates the sequence of current readings (the current pattern) that results from the two media case described in the previous chapter. In this example, medium 2, a piece

of metal $\left(\kappa_{21}>10^{7}\right)$ with a diameter $\mathrm{d}_{2}=1.9 \mathrm{~cm}$, is placed in data acquisition tank filled with saline bath, medium 1 , at $\mathrm{x}_{2}=11.6 \mathrm{~cm}$ and $\mathrm{y}_{2}=2.4$. This particular example is referred to as the reference case throughout this chapter.

Two chart types, bar and line, are presented in Figure 5-1. The bar chart presents the 15 discrete currents (one for each of the 15 electrodes) measured along $(\mathrm{x}, 0)$ side of the data acquisition tank. The $\mathrm{x}$ axis represents the electrode positions while the $\mathrm{y}$-axis represents the relative current, $\mathbf{I}_{\text {relative }}$ (equation (5-1)), defined as:

$$
\mathbf{I}_{\text {relative }}=\frac{\mathbf{I}_{\mathrm{n}}-\mathbf{I}_{\mathrm{u}}}{\mathbf{I}_{\mathrm{u}}}
$$

where $\mathbf{I}_{\text {relative }}$ is a 1 by 15 element column vector, $\mathbf{I}_{\mathrm{u}}$ are the current readings of the uniform state (i.e. saline only), and $\mathbf{I}_{\mathrm{n}}$ are the current readings of the non-uniform state (i.e. saline and other media).

The electrodes are evenly spaced along the $\mathrm{x}$ axis of the tank, thus the figure also represents the spatial distribution of the current readings. The relative current, a dimensionless quantity, is computed from difference between the non-uniform state (multi-media state) and the uniform state (single medium state) that is then divided by the uniform state current reading.

Due to the spatial and discrete nature of the current readings, the bar chart is the ideal method for displaying the current readings. However, comparing multiple current patterns using a bar chart is difficult to comprehend and interpret. Consequently, the line chart is used instead of the bar chart. The line chart aids the reader in viewing the 'pattern' that the current produces. The reader is cautioned to recall that the straight (dashed) lines connecting symbols DO NOT represent actual current values. The lines simply make it easier for the reader to perceive the current pattern. 


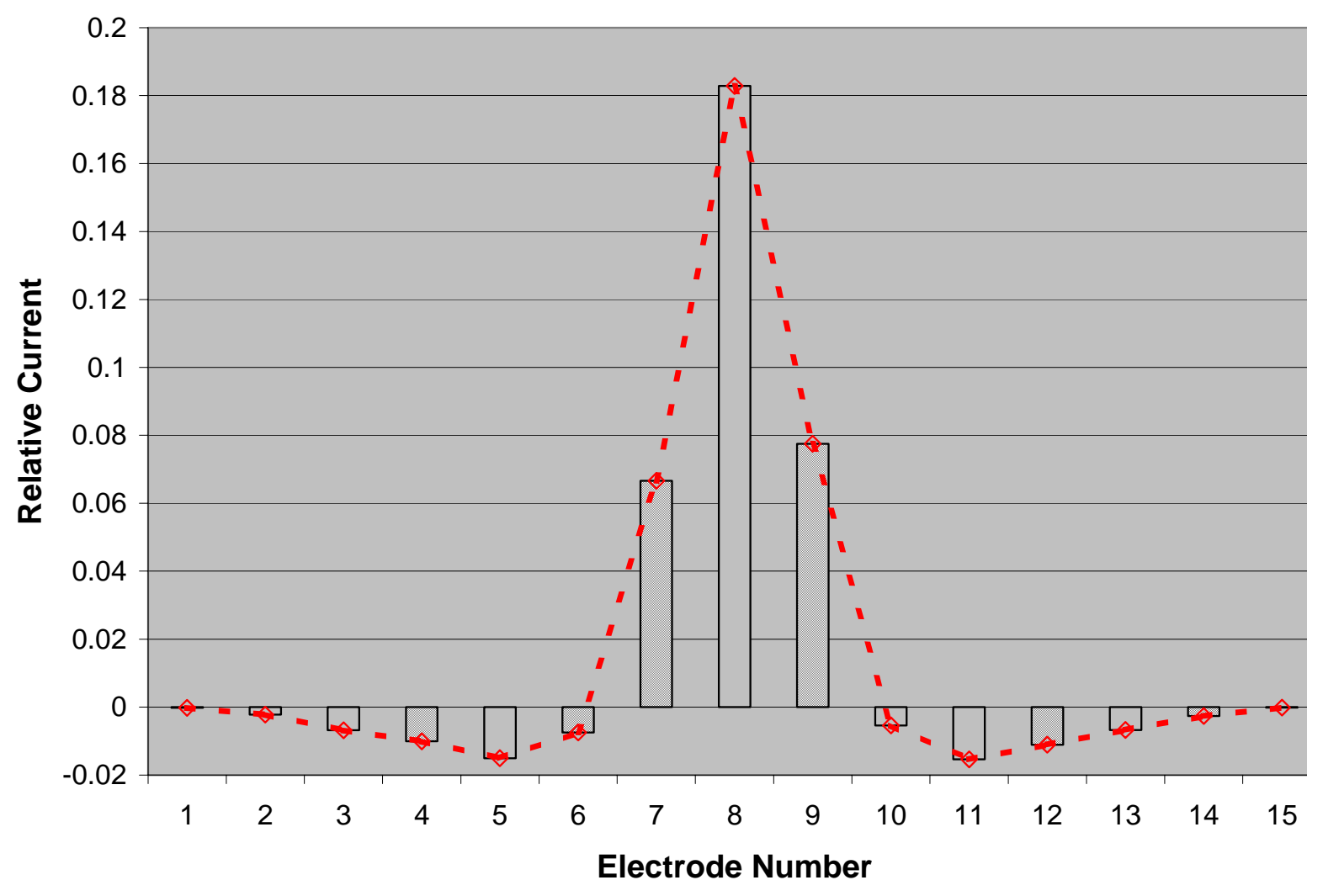

\begin{tabular}{|ll|l|l|l|l|}
\hline $\begin{array}{l}\text { Discrete Chart } \\
\text { of the relative current }\end{array}$ & $\begin{array}{l}\text { Line Chart } \\
\text { of the relative current }\end{array}$
\end{tabular}

Figure 5-1: $I_{\text {relative }}$ vs. electrode number: The reference Case

The current readings for the reference case, with medium 2 position, size, and contrast at $\mathrm{x}_{2}=11.6$ $\mathrm{cm}, \mathrm{y}_{2}=2.4 \mathrm{~cm}, \mathrm{~d}_{2}=1.9 \mathrm{~cm}$ and $\kappa_{21}=10^{7}$, are presented. The bar chart represents the discrete current readings while the line chart is used for ease of use for the reader. Note that interpolated lines between symbols do not represent actual data.

\subsubsection{Current Pattern Dependence on Position, Size and Contrast}

Figures 5-2 to 5-5 illustrate the dependence of the relative current pattern (or shape) on the position, size and contrast of medium 2. The figure $\mathrm{x}$ and $\mathrm{y}$ axes represent electrode number and relative current respectively. Each figure presents two current patterns, the reference case (same case as Figure 5-1) and the current pattern whose state results from a deviation (variation) from the reference case.

Table 5-1 below summarizes the position, size and contrast parameters for each current pattern. Figure 5-2 compares current patterns where only the lateral position, $\mathrm{x}_{2}$, varies. Figure 
5-3 compares current patterns where only the depth position, y2, varies. Figure 5-4 compares current patterns where only the size (diameter), $d_{2}$, varies. Finally, Figure 5-5 compares current patterns where only the contrast ratio varies.

Table 5-1: Position, Size, and Contrast values presented in Figures 5-2 through 5.5

\begin{tabular}{|c|c||c|c|c|c|}
\hline \multicolumn{2}{|c||}{} & \multicolumn{4}{c|}{ Position, size and contrast parameters of medium 2 } \\
\hline Case & Figure \# & $\begin{array}{c}\text { Lateral } \\
\text { Position } \\
\mathrm{x}_{2}(\mathrm{~cm})\end{array}$ & $\begin{array}{c}\text { Depth } \\
\text { Position } \\
\mathrm{y}_{2}(\mathrm{~cm})\end{array}$ & $\begin{array}{c}\text { Size } \\
\mathrm{d}_{2}(\mathrm{~cm})\end{array}$ & $\begin{array}{c}\text { Contrast } \\
\kappa_{21}\end{array}$ \\
\hline $\begin{array}{c}\text { Lateral } \\
\text { Variation }\end{array}$ & $5-2$ & 8.5 & 2.4 & 1.9 & $>10^{7}$ \\
$\begin{array}{c}\text { Depth } \\
\text { Variation }\end{array}$ & $5-3$ & 11.6 & 3.9 & 1.9 & $>10^{7}$ \\
\hline $\begin{array}{c}\text { Size } \\
\text { Variation }\end{array}$ & $5-4$ & 11.6 & 2.4 & 1.6 & $>10^{7}$ \\
\hline $\begin{array}{c}\text { Contrast } \\
\text { Variation }\end{array}$ & $5-5$ & 11.6 & 2.4 & 1.9 & 7.4 \\
\hline
\end{tabular}

Note: $\sigma_{1}=0.08 \mathrm{~S} / \mathrm{m}$ for the background saline solution.

In general terms, each current pattern displays a Gaussian-like shape with the relative current peak corresponding to the lateral position ( $\mathrm{x}_{2}$ ) of medium 2 (Figure 5-2). As the depth position $\left(\mathrm{y}_{2}\right)$ increases, the magnitude of the relative current peak decreases and the Gaussian shape broadens (Figure 5-3). Finally, as the size $\left(\mathrm{d}_{2}\right)$ and contrast $\left(\kappa_{21}\right)$ decrease, the magnitude of the relative current peak decreases, but the Gaussian shape is maintained i.e. the magnitude of all 15 current readings changes by the same proportion. These observations suggest the relative current pattern is a function of lateral position, depth position, size, and contrast. This relationship is expressed in functional form (equation (5-2)) as:

$$
\mathbf{I}_{\text {relative }}=\mathbf{I}\left(x_{2}, y_{2}, d_{2}, \kappa_{21}\right)
$$

where $\mathrm{x}_{2}$ is the lateral position,

$\mathrm{y}_{2}$ is the depth position,

$\mathrm{d}_{2}$ is the diameter, and

$\kappa_{21}$ is the contrast ratio $\left(\kappa_{21}=\frac{\sigma_{2}}{\sigma_{1}}\right)$ of medium 2 . 


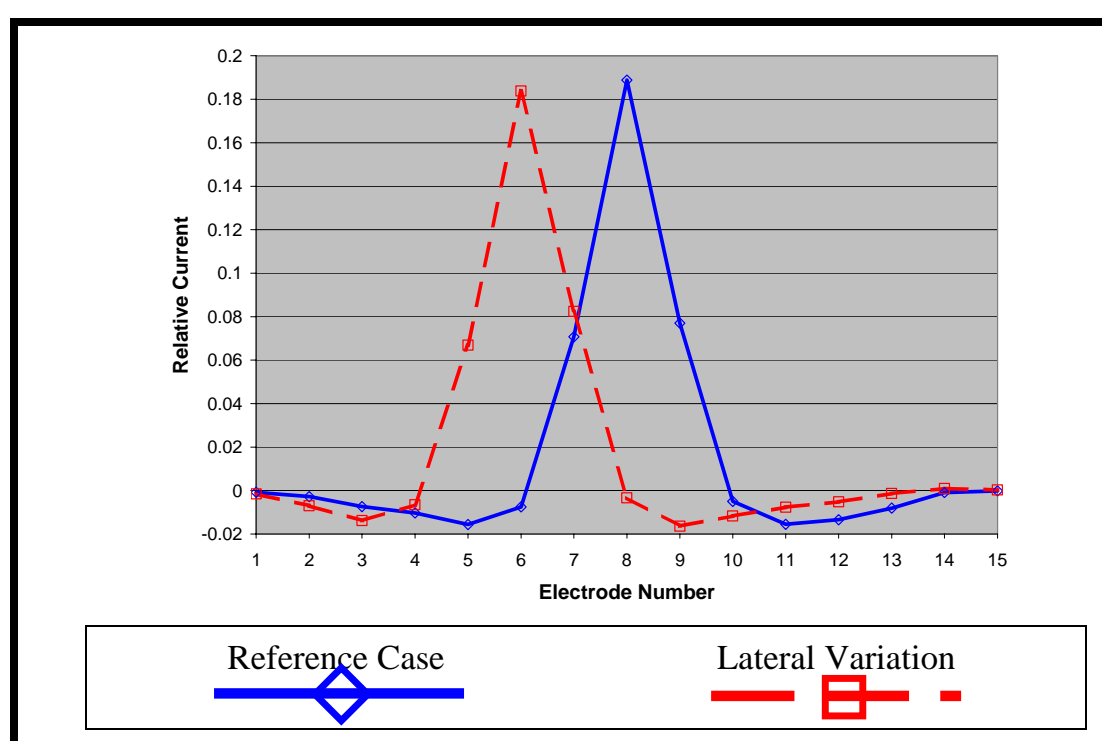

Figure 5-2: $I_{\text {relative }}$ vs. electrode number: Lateral position dependence Current patterns for the reference and laterally varied cases $\left(\mathrm{x}_{2}=11.6\right.$ and $8.5 \mathrm{~cm}$ respectively) are presented.
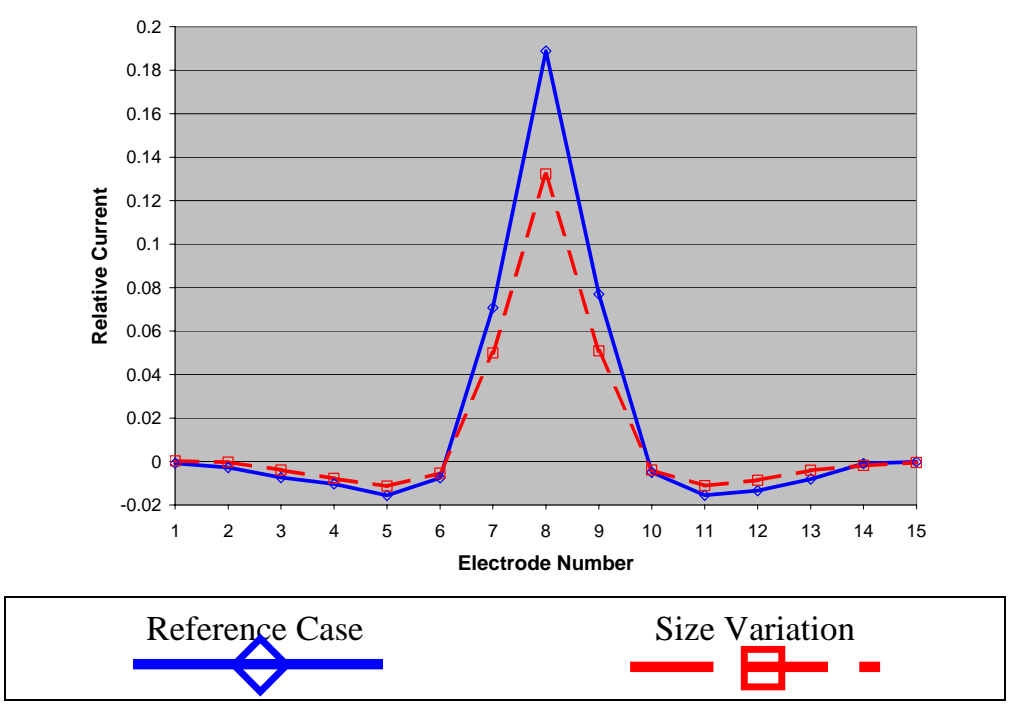

Figure 5-4: $I_{\text {relative }}$ vs. electrode number: Size dependence:

Current patterns for the reference and size varied cases $\left(\mathrm{d}_{2}=1.9\right.$ and 1.3 $\mathrm{cm}$ respectively) are presented.
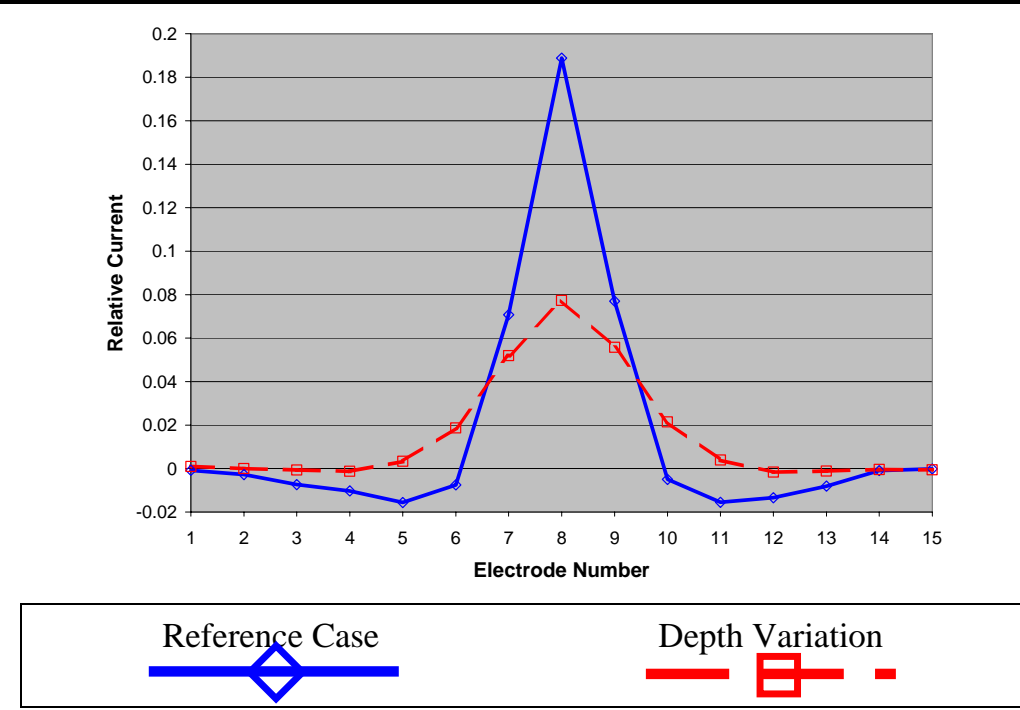

Figure 5-3: $I_{\text {relative }}$ vs. electrode number: Depth position dependence Current patterns for the reference and depth varied cases $\left(\mathrm{y}_{2}=2.4\right.$ and $5.5 \mathrm{~cm}$ respectively) are presented.

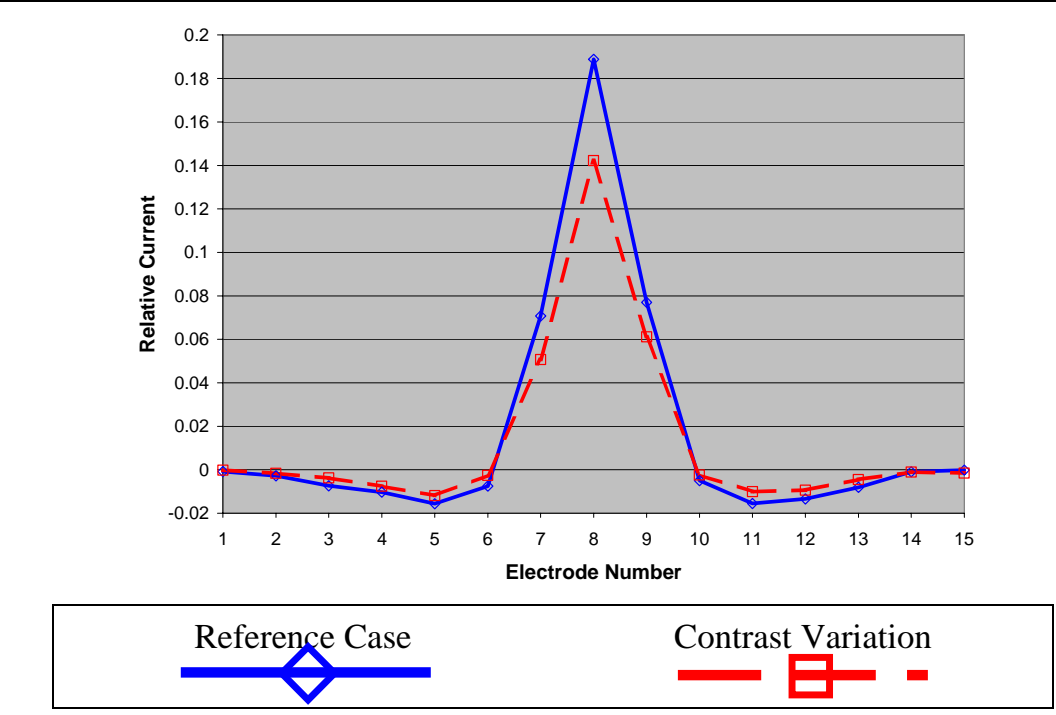

Figure 5-5: $I_{\text {relative }}$ vs. electrode number: Contrast dependence

Current patterns for the reference and contrast varied cases $\left(\kappa_{21}=10^{7}\right.$ and 7.4 respectively) are presented. 


\subsubsection{Quantifying the Similarity in Current Pattern Shape}

The similarity between the shapes of two current patterns is quantified using the crosscorrelation function $\mathrm{r}\left(\mathbf{I}^{\mathrm{R}}, \mathbf{I}^{\mathrm{T}}\right)$ (equation (5-3)) [156]. The cross-correlation is a statistical function that measures how well two current patterns, $\mathbf{I}^{\mathrm{R}}$ and $\mathbf{I}^{\mathrm{T}}$, vary jointly. Cross correlation values range from -1 to +1 . Values near $r= \pm 1$ indicate a high degree of correlation and a good fit to a linear model. A value of $r$ close to 0 indicates a poor fit to a linear model.

$$
r\left(\mathbf{I}^{\mathrm{R}}, \mathbf{I}^{\mathrm{T}}\right)=\frac{\sum_{k=1}^{15}\left(\mathrm{I}_{k}^{\mathrm{R}}-\overline{\mathrm{I}}^{\mathrm{R}}\right) \cdot\left(\mathrm{I}_{k}^{\mathrm{T}}-\overline{\mathrm{I}}^{\mathrm{T}}\right)}{\sqrt{\sum_{k=1}^{15}\left(\mathrm{I}_{k}^{\mathrm{R}}-\overline{\mathrm{I}}^{\mathrm{R}}\right)^{2} \sum_{k=1}^{15}\left(\mathrm{I}_{k}^{\mathrm{T}}-\overline{\mathrm{I}}^{\mathrm{T}}\right)^{2}}}
$$

where $I^{R}$ is the current pattern for the reference state

$\mathrm{I}^{\mathrm{T}}$ is the current pattern for the test state

$\overline{\mathrm{I}}$ is the mean current value

$\mathrm{k}$ is the index corresponding to the electrode position

The cross correlation is computed from the reference and test current patterns $\left(\mathbf{I}^{\mathrm{R}}\right.$ and $\mathbf{I}^{\mathrm{T}}$ respectively). The superscripts $\mathrm{R}$ and $\mathrm{T}$ refer to the state that produces the current values. The reference current pattern is defined in equation (5-4). The test current pattern is defined similarly.

$$
\mathbf{I}^{\mathrm{R}}=\mathbf{I}\left(x_{2}^{R}, y_{2}^{R}, d_{2}^{R}, \kappa_{21}^{R}\right)
$$

Cross-correlation analysis is applied to the experimental results where one parameter is varied while leaving the remaining parameters unchanged. These results are presented in tables 5-2 through 5-4. Table 5-2 examines how similar the shape of the current pattern is for different depths $\left(\mathrm{y}_{2}\right)$ of medium 2 (equation (5-5)). Table 5-3 examines shape similarity as the size (diameter) $d_{2}$ of medium 2 varies (equation (5-6)). Finally, Table 5-4 examines shape similarity as the contrast, $\kappa_{21}$, of medium 2 varies (equation (5-7)). The lateral dependence on the shape of the current pattern is not tested since it obviously changes with the lateral position. 


$$
\begin{aligned}
& r\left(\mathbf{I}^{R}, \mathbf{I}^{T}\right)=r\left(\mathbf{I}\left(x^{R}, y^{R}, d^{R}, \kappa^{R}\right), \mathbf{I}\left(x^{R}, y^{T}, d^{R}, \kappa^{R}\right)\right) \\
& r\left(\mathbf{I}^{R}, \mathbf{I}^{T}\right)=r\left(\mathbf{I}\left(x^{R}, y^{R}, d^{R}, \kappa^{R}\right), \mathbf{I}\left(x^{R}, y^{R}, d^{T}, \kappa^{R}\right)\right) \\
& r\left(\mathbf{I}^{R}, \mathbf{I}^{T}\right)=r\left(\mathbf{I}\left(x^{R}, y^{R}, d^{R}, \kappa^{R}\right), \mathbf{I}\left(x^{R}, y^{R}, d^{R}, \kappa^{T}\right)\right)
\end{aligned}
$$

The tables are laid out in a matrix format. For instance, the left most column in Table 5-2 lists the depth $y_{2}^{\mathrm{R}}$ for the reference case, while the top row lists the depth $y_{2}^{\mathrm{T}}$ of the test case. Each element of the matrix contains the cross-correlation coefficient corresponding to the combination of reference depth and test depth. Tables 5-3 and 5-4 are laid out in a similar

\begin{tabular}{|c|c|c|c|c|c|c|}
\hline \multirow{2}{*}{$\begin{array}{l}\text { Reference } \\
\text { depth } \\
\text { position } \\
\mathrm{y}_{2}^{\mathrm{R}}(\mathrm{cm}) \\
\end{array}$} & \multicolumn{6}{|c|}{ Test depth position $\mathrm{y}_{2}^{\mathrm{T}}(\mathrm{cm})$} \\
\hline & 2.4 & 3.9 & 5.5 & 7.0 & 8.5 & 11.6 \\
\hline 2.4 & 1.000 & & & & & \\
\hline 3.9 & 0.926 & 1.000 & & & & \\
\hline 5.5 & 0.819 & 0.969 & 1.000 & & & \\
\hline 7.0 & 0.730 & 0.910 & 0.976 & 1.000 & & \\
\hline 8.5 & 0.638 & 0.838 & 0.936 & 0.983 & 1.000 & \\
\hline 11.6 & 0.538 & 0.740 & 0.866 & 0.927 & 0.970 & 1.000 \\
\hline
\end{tabular}
fashion.

Table 5-2: Cross-correlation coefficients as a function of depth for medium 2

Table 5-3: Cross-correlation coefficients as a function of size for medium 2

\begin{tabular}{|c||c|c|c|c|}
\hline \multicolumn{1}{|c||}{ Reference size $\mathrm{d}_{2}^{\mathrm{R}}(\mathrm{cm})$} & \multicolumn{4}{|c|}{ Test size $\mathrm{d}_{2}^{\mathrm{T}}(\mathrm{cm})$} \\
\cline { 2 - 5 } & 1.0 & 1.3 & 1.6 & 1.9 \\
\hline \hline 1.0 & 1.000 & \multicolumn{3}{c}{} \\
\hline 1.3 & 0.999 & 1.000 & \\
\hline 1.6 & 0.999 & 0.998 & 1.000 & \\
\hline 1.9 & 0.999 & 0.999 & 1.000 & 1.000 \\
\hline
\end{tabular}


Table 5-4: Cross-correlation coefficients as a function of contrast for medium 2

\begin{tabular}{|c|c|c|c|c|c|}
\hline Reference contrast $\kappa_{21}^{\mathrm{R}}$ & \multicolumn{5}{|c|}{ Test contrast $\kappa_{21}^{\mathrm{T}}$} \\
\cline { 2 - 5 } & 2.4 & 4.4 & 7.4 & 15.2 & $\begin{array}{c}\text { Metal } \\
\left(>10^{7}\right)\end{array}$ \\
\hline \hline 2.4 & 1.000 & \multicolumn{3}{|c|}{} \\
\hline 4.4 & 0.999 & 1.000 & 1.000 & \\
\hline 7.4 & 0.999 & 0.999 & 1.000 & 1.000 & \\
\hline 15.2 & 0.999 & 1.000 & 1.000 & 000 \\
\hline Metal $\left(>10^{7}\right)$ & 0.999 & 0.999 & 1.000 & 1.000 & 1.000 \\
\hline
\end{tabular}

Note: $\sigma_{1}=0.08 \mathrm{~S} / \mathrm{m}$

In Table 5-2, the cross-correlation value when the reference and test depths are the same is1.000 (as expected). The cross correlation value decreases as the distance between the reference and test depths increases indicating that the current pattern shape is a function of the depth. The cross-correlation coefficients in tables $5-3$ and $5-4$ do NOT vary appreciably with variations in the size or contrast ( $\mathrm{d}_{2}$ or $\left.\kappa_{21}\right)$ of medium 2 . The coefficient is approximately 1.0 indicating that the current pattern shape (but not magnitude) is independent of the size or contrast of medium 2.

\subsubsection{Examining Current Magnitude Dependence on Contrast}

Cross-correlation analysis demonstrates that the shape of the current pattern remains unchanged as the contrast is varied. The charge-charge correlation theory from Chapter 3 predicts the magnitude of the current pattern is a scalar multiple of the current pattern where medium 2 is composed of metal (equation (3-47)). This scalar multiple, the scaling factor $\xi$, is postulated to be a function of the contrast ratio $\kappa_{21}$ (equation (3-48)). Experimental results from experimental group 4 are used as an example for testing whether the current pattern magnitude does indeed match predictions.

$$
\mathbf{I}^{\text {non-metal }}=\xi \cdot \mathbf{I}^{\text {metal }}
$$

where $\xi$, the scaling factor, is computing using standard regression techniques

$$
\xi=\frac{\kappa-1}{\kappa+1}
$$


Figure 5-6 compares computed and predicted scaling factor vs. contrast from experimental group 4 data. Experimental scaling factor values are computing using the relationship between high contrast and low contrast current patterns presented in equation (5-8) (originally equation (3-47)). The predicted scaling factor is computed from the relationship presented in equation (5-9) (originally equation (3-48)). The experiments were conducted with 24 separate sets of contrast ratios ranging from nearly 1.0 to 15.0. These experimental scaling factor results (diamonds) are plotted vs. the known contrast ratio while the predicted scaling factor function is overlaid as a solid line. The error bars represent \pm 1 standard deviation about the mean of the computed scaling factor values.

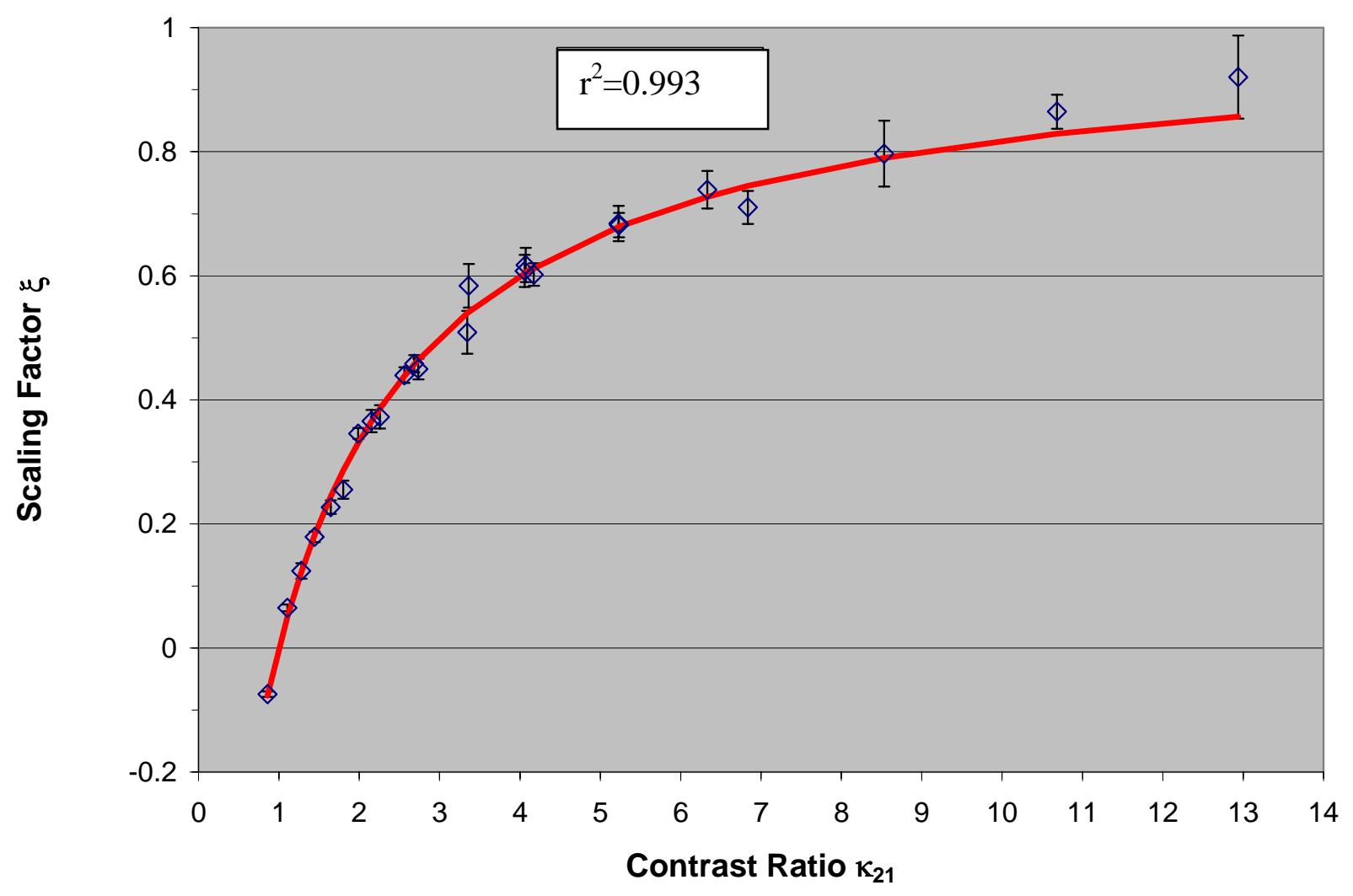

Experimental scaling factor Values

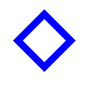

Predicted scaling factor function

Figure 5-6: Experimental and predicted scaling factor results: $\xi$ vs. $\kappa_{21}$

Predicted and experimentally determined scaling factors are plotted versus contrast ratio. Experimentally computed scaling factors are presented with uncertainty bars that are \pm 1 standard deviation of the mean. The coefficient of determination between the predicted and experimental results was computed with a value of 0.993 . 
The goodness of fit between the predicted and computed (from experimental data) scaling factor is quantified using the coefficient of determination, $\mathrm{r}^{2}$. The coefficient of determination is computed from the square of the correlation. Values range from 0 to 1 , where values close to 1 indicate there is no difference between the estimated scaling factor-value and the actual scaling factor-value while values close to 0 indicate the model is not helpful in predicting a scaling factor-value. The coefficient of determination indicates how much of the total variation in the dependent variable can be accounted for by the predicted scaling factor function. Most statisticians consider a COD of .7 or higher for accepting the model as reasonable [157]. The coefficient of determination is 0.993 , which experimentally supports the notion that magnitude of the current pattern is predictable.

$$
\text { c.v. }=\frac{\text { standard deviation of } \xi}{\text { mean of } \xi} \times 100 \%
$$

An interesting observation is that the error bars in Figure 5-6 appear to increase in magnitude as the scaling factor increases in magnitude. To determine if this is an issue to be concerned about, the coefficient of variance is computed. The coefficient of variance, sometimes called the relative standard deviation, is the degree to which a set of data points varies. It is defined as the ratio of the standard deviation to the mean (equation (5-10)), denoted as c.v. and quoted in percentage [156]. The c.v. vs. scaling factor results are presented in Figure 5-7. Except for two outlier points, the c.v. values generally fall between 3 and $7 \%$. The c.v. results suggest that the error bars presented in Figure 5-6 are consistent and not abnormal. 


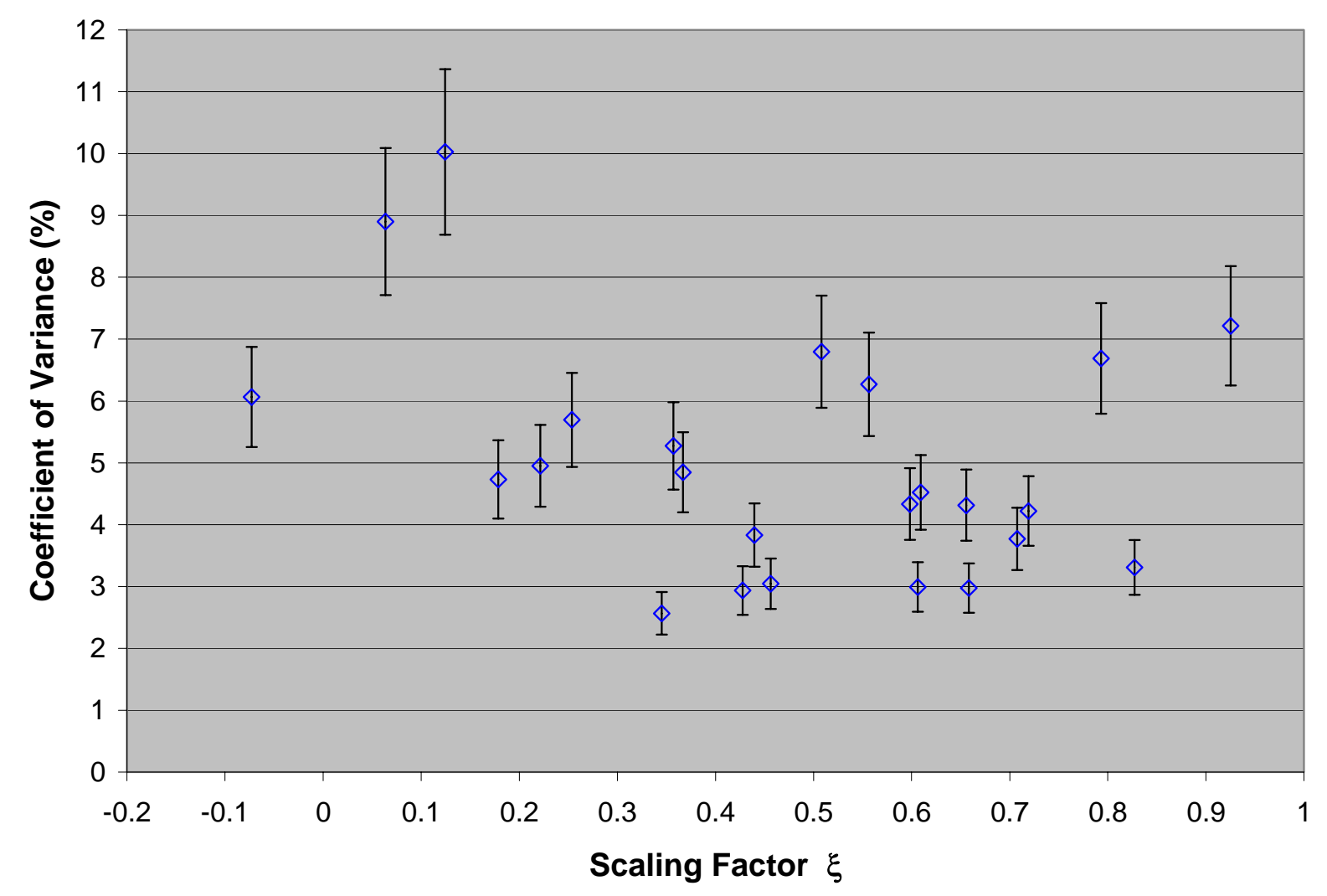

Figure 5-7: Coefficient of variance vs. scaling factor for experimental results

The coefficient of variance for the experimentally determined scaling factors is plotted versus contrast ratio. The uncertainty bars are \pm 1 standard deviation of the c.v.

\subsection{Forward Solution Results}

The next section determines if the high contrast forward solution (equation (3-45)) accurately fits the experimental result that is necessary in determining the contrast ratio, $\kappa_{21}$. The predicted current pattern, computed from the high contrast forward solution, is applied to data from experimental groups 1, 2 and 3. These experiments all use a medium 2 composed of metal.

\subsubsection{Comparing Measured and Predicted Current Patterns}

Figure 5-8 compares the predicted high contrast forward solution with the measured relative current results for the reference case $\left(x_{2}=11.6 \mathrm{~cm}, \mathrm{y}_{2}=2.4 \mathrm{~cm}, \mathrm{~d}_{2}=1.9 \mathrm{~cm}, \kappa_{21}=10^{7}\right)$. The $\mathrm{x}$ - and $\mathrm{y}$ - axes represent the electrode number and relative current respectively. Solid lines with 
diamond symbols represent experimental results while dashed lines with square symbols represent predicted lines. Visually, the predicted and measured current patterns appear to have nearly identical values for all 15 data points. The coefficient of determination $\left(r^{2}\right)$, which is 0.999, confirms this assessment. The high contrast forward solution is deemed a reasonable fit for the reference case.

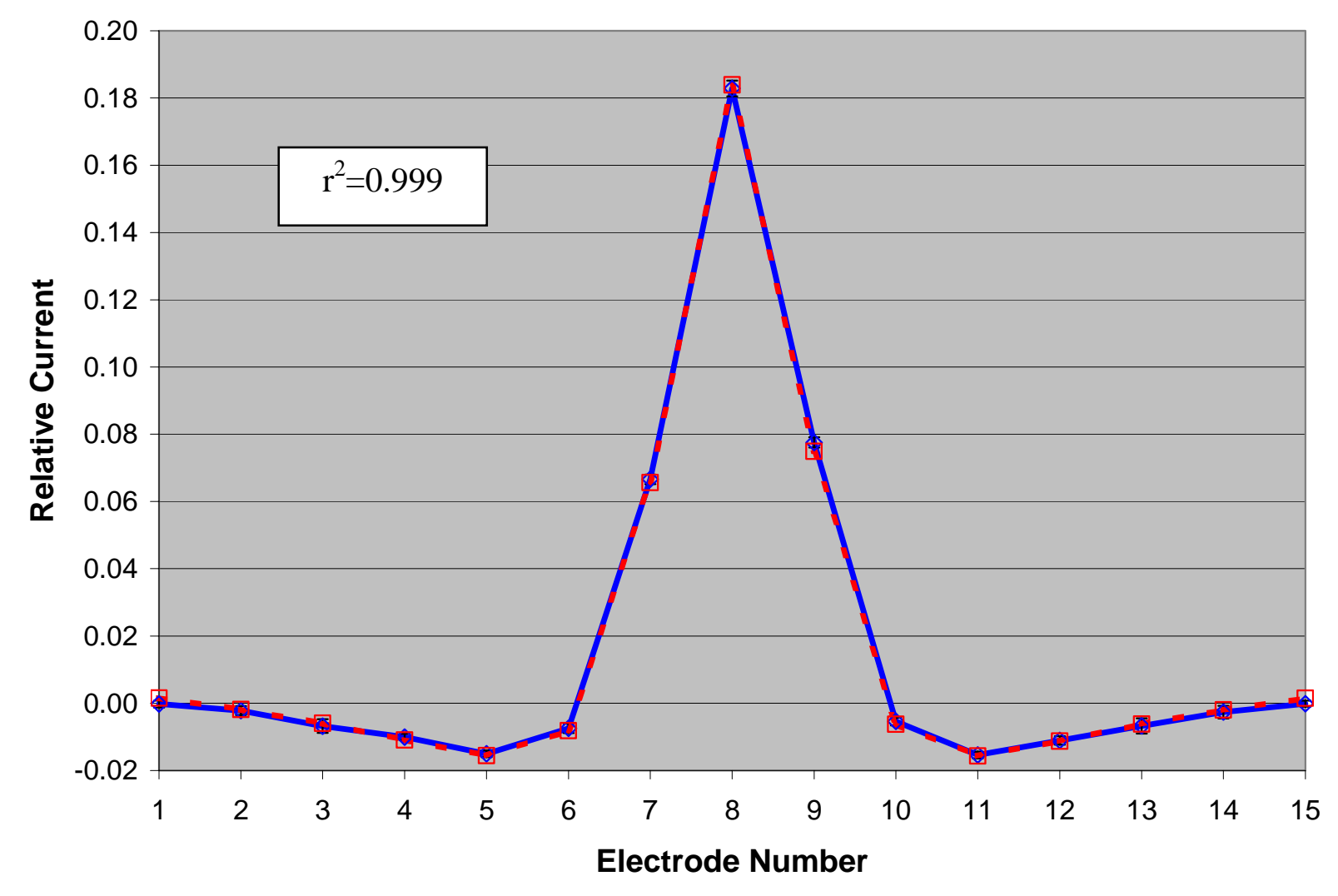

Experimental Current Pattern

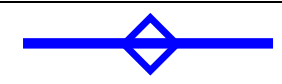

Predicted Current

Pattern

Figure 5-8: $I_{\text {relative }}$ vs. electrode number: Reference case measured and predicted

The predicted and measured current patterns (dashed and solid lines respectively) for the reference case with position, size, and contrast at $\mathrm{x}_{2}=11.6 \mathrm{~cm}, \mathrm{y}_{2}=2.4 \mathrm{~cm}, \mathrm{~d}_{2}=1.9 \mathrm{~cm}$ and $\kappa_{21}=10^{7}$ are presented. The coefficient of determination was determined to be 0.999 .

The high contrast forward solution is applied to all experimental results from experimental groups 1,2 , and 3 . Recall that experimental group 1 experiments vary lateral position, experimental group 2 experiments vary depth position, and experimental group 3 experiments vary size, all with respect to the reference case. In all cases the coefficient of 
determination is at least 0.9. Consequently, the forward solution is deemed an accurate model for predicting the current patterns for all cases under study.

\subsubsection{Estimating Limits on the Forward Solution}

This section examines the question, "at what stage does the forward solution stop yielding useful information?” The behavior of the high contrast forward solution is characterized using a single experimental data point in comparison with the corresponding predicted point. Since the coefficient of determination, $\mathrm{r}^{2}$, is close to one, comparing single corresponding data points is reasonable. An obvious point to compare is the peak current (i.e. the current with the maximum value) defined in equation (5-11).

$$
\mathrm{I}_{\text {peak }}=\max (\operatorname{abs}(\mathbf{I}))
$$

The peak current value is plotted for lateral position, depth position, and size variations (figure 5-9 through 5-11). Figure 5-9 presents the peak current resulting from the placement of medium 2 (composed of metal) at various lateral positions ranging from $1.7 \mathrm{~cm}$ to $11.6 \mathrm{~cm}$ lateral position (experimental group 1 results). The $\mathrm{x}$-axis represents the lateral position $\left(\mathrm{x}_{2}\right)$ in $\mathrm{cm}$ while the y-axis represents the peak current. Experimental peak current is plotted with diamond symbols, with 14 separate points corresponding to 14 separate placement positions. Predicted peak current is plotted with a solid line.

Recall that the peak current is selected from 1 of 15 electrodes. The electrode corresponding to the peak current depends on the lateral location of medium 2. The predicted and experimental results display oscillatory behavior. This is expected. Recall from Figure 5-1, the current is measured at a discrete number of electrodes with a discrete size. If the current pattern were measured with an infinite number of electrodes, the discrete current pattern would appear as a continuous current pattern. With only 15 electrodes, the current pattern is 'discretized.' If the peak current is located directly between two electrodes, the two adjacent electrodes will share the discretized current pattern, thus the current will appear to decrease. This result, though, is not primary to the issue at hand. The reader is directed to the Appendix C for a discussion on the reasons for this oscillatory behavior. 


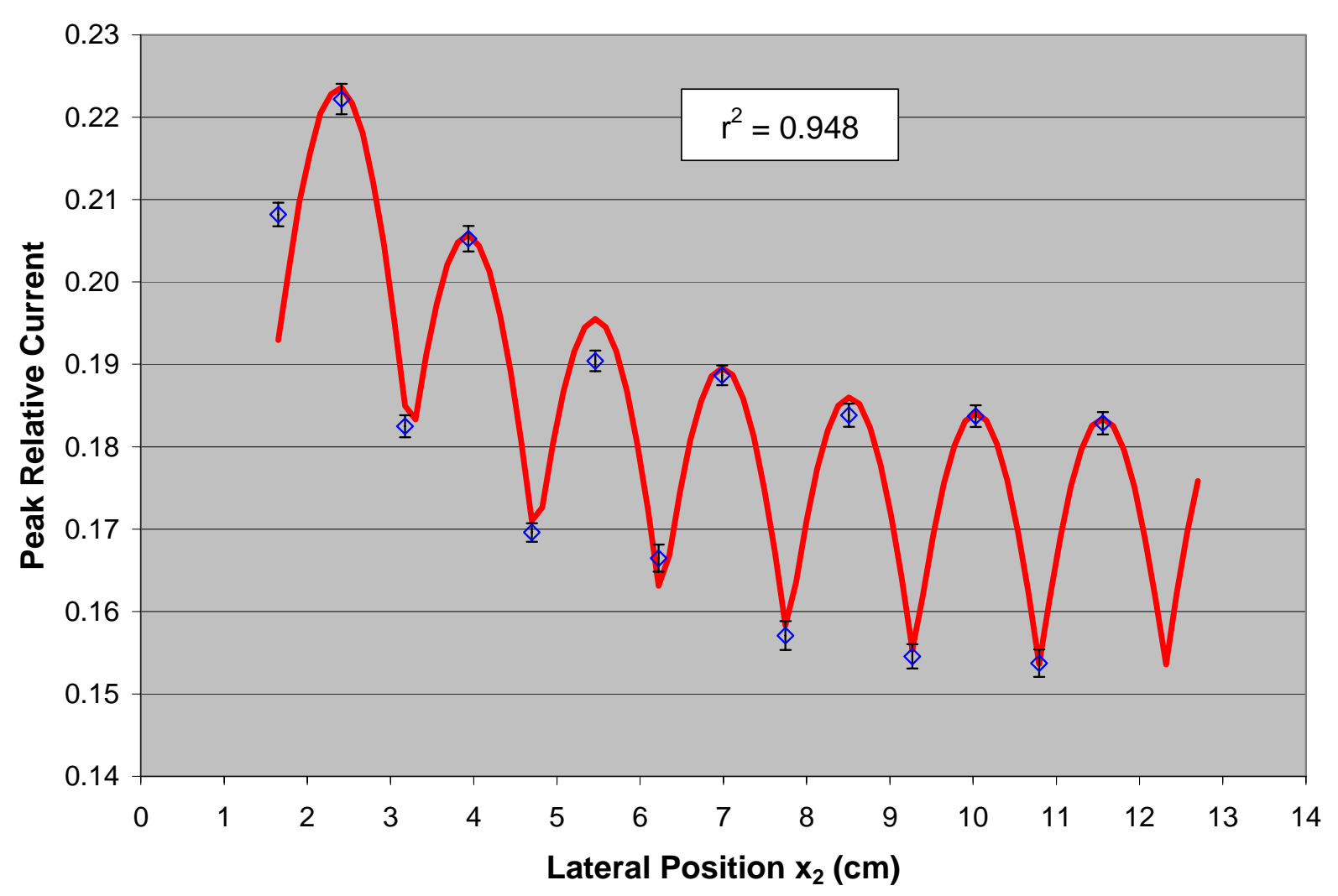

Experimental Peak Currents

Predicted Peak Current

Figure 5-9: Peak $I_{\text {relative }}$ vs. lateral position $x_{2}$

Measured and predicted peak current (diamond symbol and solid line respectively) as a function of the lateral position of medium 2.

The predicted and experimental results display good agreement and the coefficient of determination is 0.948 . The high contrast forward solution accurately predicts the peak relative current. The selection of the uniform case potential in equations (3-37) and (3-38) to compute internal charge is a reasonable approximation of the internal potential for small sized media. 


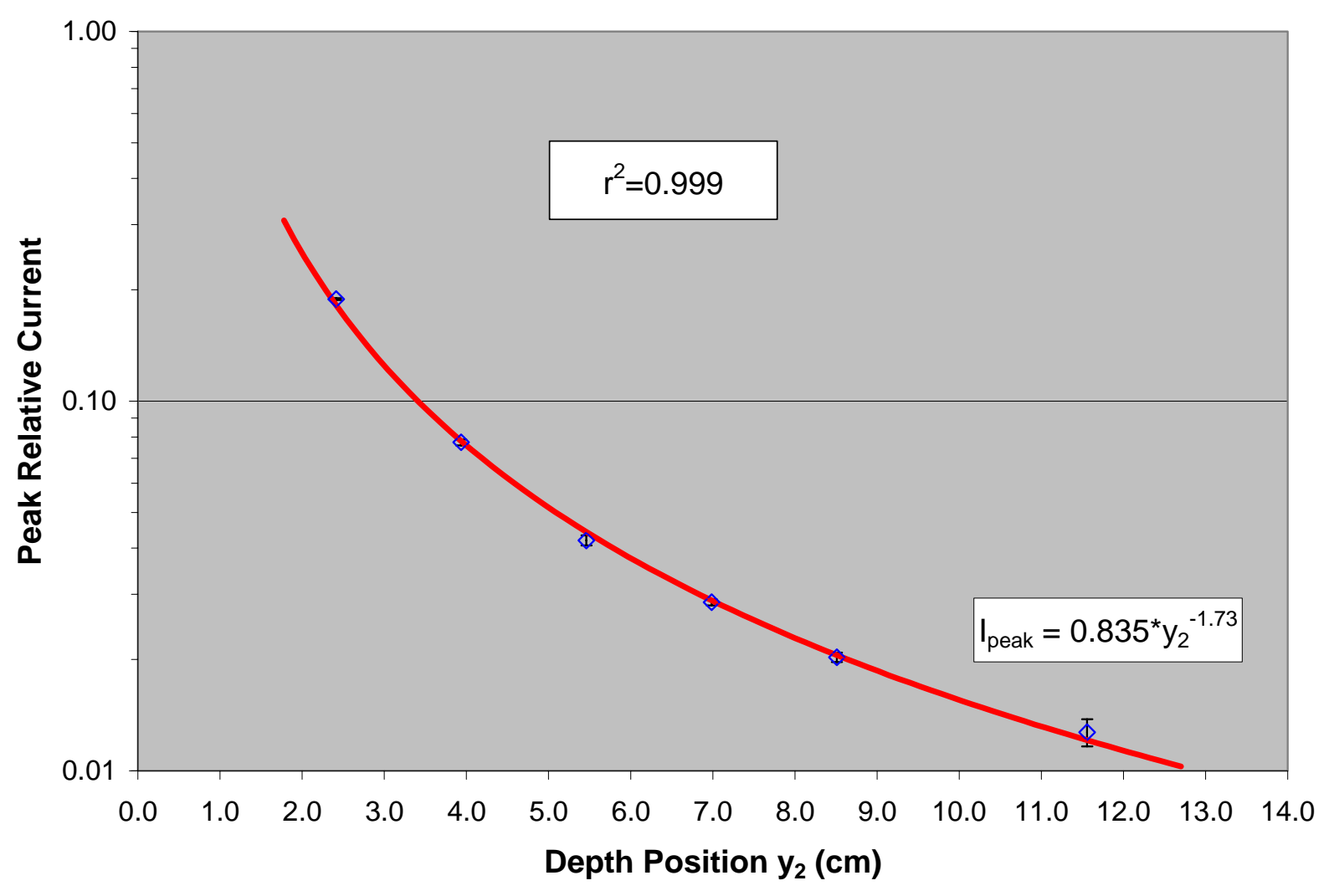

Experimental Peak Currents $\quad$ Predicted Peak Currents

Figure 5-10: Peak $I_{\text {relative }}$ vs. depth position $y_{2}$

Measured and predicted peak current (diamond symbol and solid line respectively) as a function of the depth position of medium 2. The predicted peak current was fit to a power law function.

Figure 5-10 presents the peak current resulting from the placement of medium 2 at six different depth positions ranging from $\mathrm{y}_{2}=2.4 \mathrm{~cm}$ to $11.6 \mathrm{~cm}$ depth position (experimental group 2 results). The $\mathrm{x}$-axis represents the depth position $\left(\mathrm{y}_{2}\right)$ in $\mathrm{cm}$ while the y-axis represents the peak current. Experimental peak current is plotted with diamond symbols and predicted peak current is plotted with a solid line.

The predicted and experimental results display nearly perfect agreement and the coefficient of determination is 0.999 . The high contrast forward solution accurately predicts the peak relative current. The experimental and predicted results decline with increasing depth. This behavior is characterized with a power series approximation (equation (5-12)). 


$$
I_{\text {peak }}=0.835 \cdot y_{2}^{-1.73}
$$

Figure 5-11 presents the peak current resulting from the placement of medium 2 for four different sizes ranging from $\mathrm{d}_{2}=1.0$ to $1.9 \mathrm{~cm}$ diameter (experimental group 3 results). The $\mathrm{x}$ axis represents the size $\left(d_{2}\right)$ in $c m$ while the y-axis represents the peak current. Experimental peak current for experimental results is plotted with diamond symbols and predicted peak current is plotted with a solid line.

$$
I_{\text {peak }}=0.053 \cdot d_{2}^{1.94}
$$

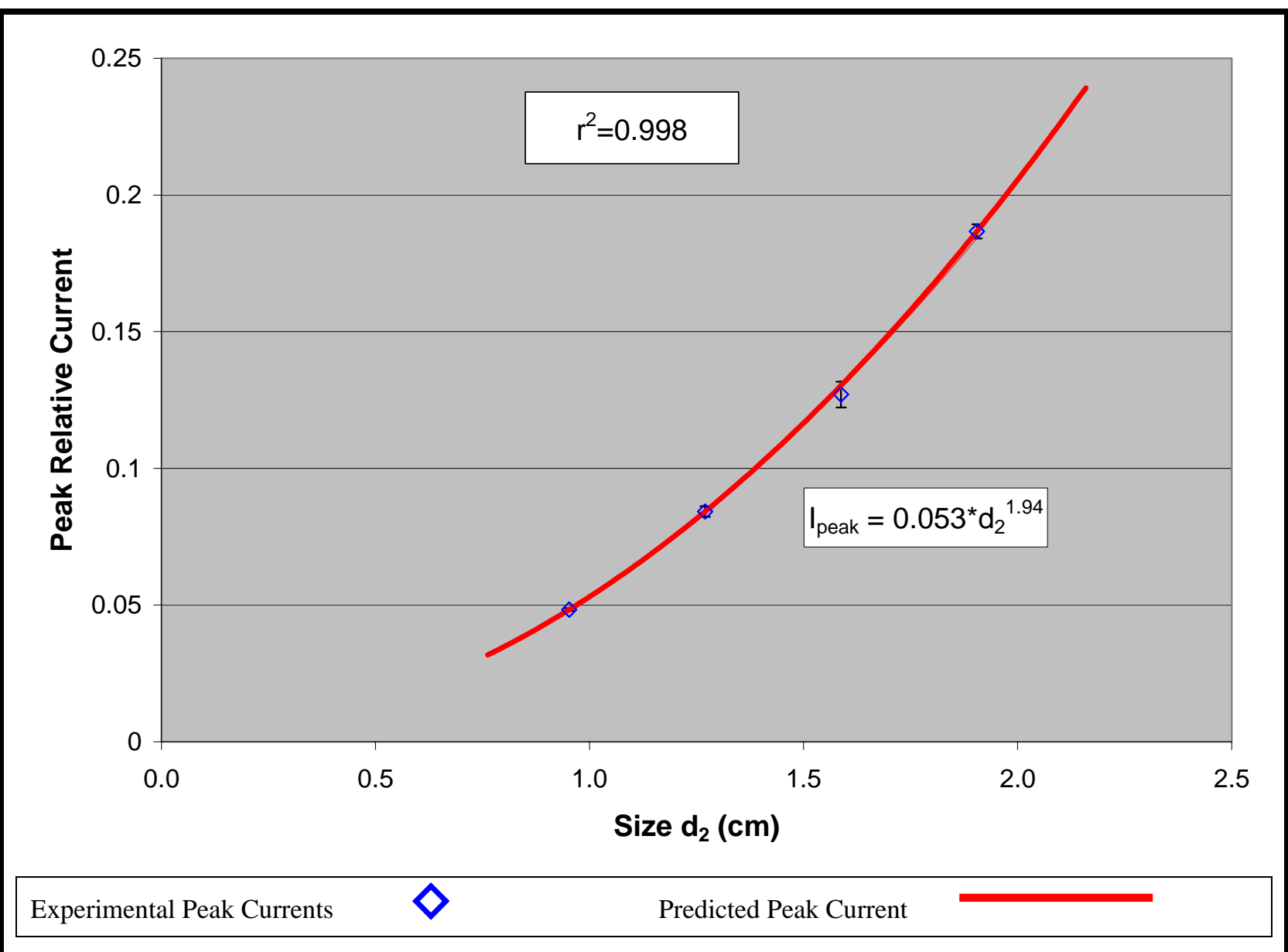

Figure 5-11: Peak $I_{\text {relative }}$ vs. size $d_{2}$

Measured and predicted peak current (diamond symbol and solid line respectively) as a function of the size of medium 2. The predicted peak current was fit to a power law function.

The predicted and experimental results display nearly perfect agreement and the coefficient of determination is 0.998 . The high contrast forward solution accurately predicts the 
peak relative current. The experimental and predicted results decline with decreasing size. This behavior is characterized with a power series approximation (equation (5-13)).

$$
\begin{gathered}
0.0034=\left(\frac{\kappa_{21}-1}{\kappa_{21}+1}\right) \cdot\left(0.835 \cdot(11.6)^{-1.73}\right) \cdot \frac{0.053 \cdot d_{2}^{1.94}}{0.053 \cdot(2.4)^{1.94}} \\
d_{2}=\left(0.636 \cdot\left(\frac{\kappa_{21}-1}{\kappa_{21}+1}\right)\right)^{-1 / 1.94}
\end{gathered}
$$

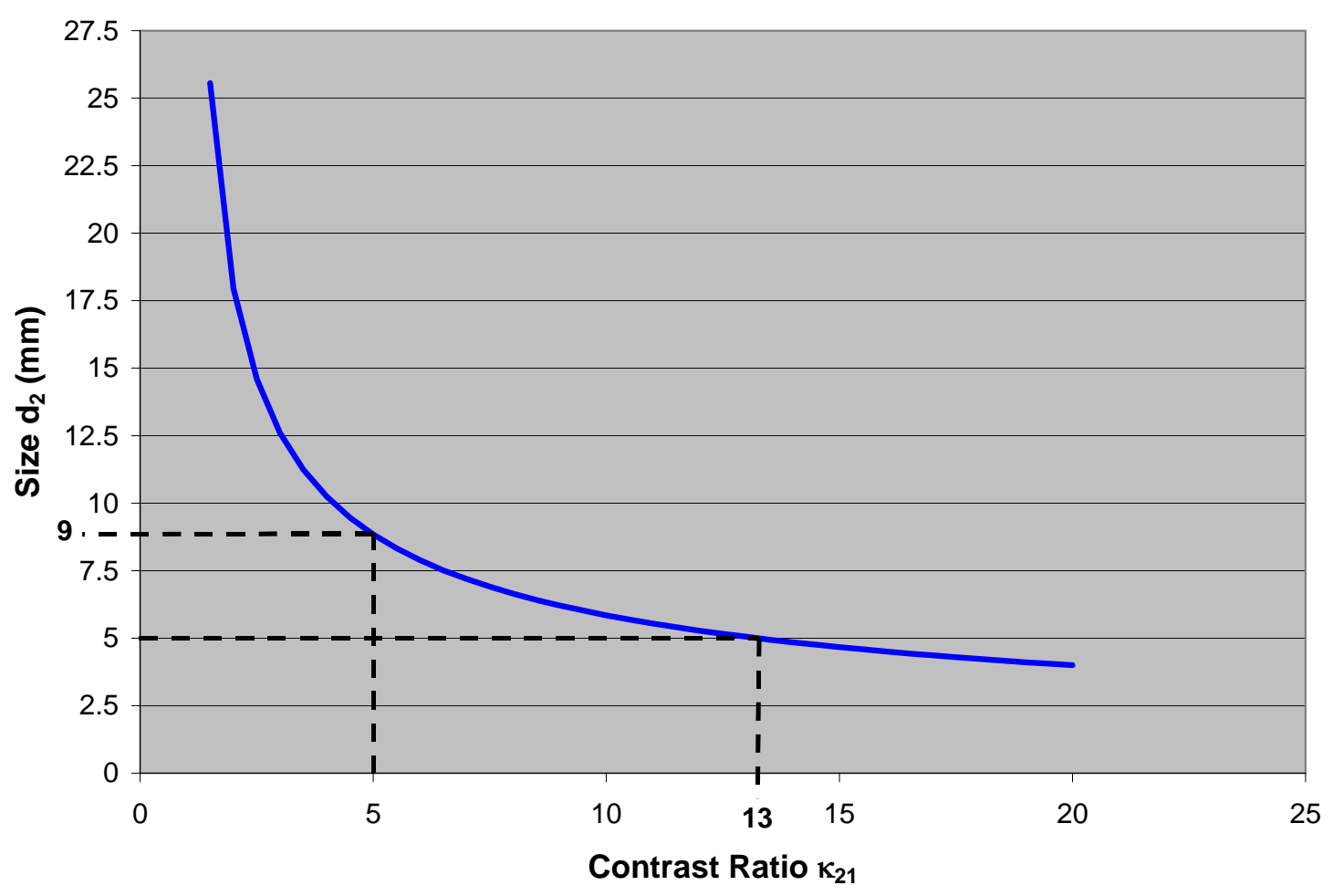

Figure 5-12: Determining minimum size and contrast detectability

The balance between minimum detectable size vs. minimum detectable contrast for medium 2 located at the center of the data acquisition tank $\left(\mathrm{y}_{2}=11.6 \mathrm{~cm}\right)$ is presented here. For instance, to detect a $5 \mathrm{~mm}$ object the contrast must be greater than 13 . Alternatively, to detect an object with a contrast of 5 , the size must be greater than $9 \mathrm{~mm}$.

The final step is to determine the limit on the maximum depth, minimum size, and minimum contrast ratio that can be reasonably detected. At some given depth and size, the current pattern signal is lost in random signal noise. This noise is unique for each data acquisition system. This noise, the noise floor, is estimated for this system using the standard error of the estimate [156] to be 0.0034 (in units of relative current). Based on symmetry 
arguments, the most distant point in the data acquisition tank to place medium 2 is the tank center, $\mathrm{y}_{2}=11.6 \mathrm{~cm}$. To determine a relationship between the minimum size and contrast that is detectable at the tank center, the noise floor, 0.0034, the relationships expressed in equations (5-12) and (5-13), along with the scaling factor relationship in equation (3-48) are substituted into (3-47) to produce equation (5-14). The depth position is chosen to be $y 2=11.6 \mathrm{~cm}$. Equation (5-14) is rearranged to form an expression relating size to contrast (equation (5-15)) and is presented in Figure 5-12. For instance, to detected an object with a diameter less than $5 \mathrm{~mm}$, the contrast ratio must be greater than 13. Alternatively, to detect and object with a contrast ratio less than 5 , the object must have a diameter greater than $9 \mathrm{~mm}$.

\subsection{Inverse Solution Results}

The third section presents the conductivity (contrast) results predicted by the inverse method. The section is divided into three separate groups. The first group presents data for the two media experiments (experimental groups 5 and 6). The second group presents results for the three media experiments that use only agar for medium 2 and 3 (experimental groups 6 and 7). The final group presents experimental group 9 results that are for more complex experiments using liver and oatmeal. The results in this final group are intended to shed some light on the future direction this research might eventually take. Predicted results for the first and second groups are computed using the charge-charge correlation method. Predicted results for the third group are computed using the indirect reconstruction method.

\subsubsection{Two Media Results: Predicted Contrast vs. Depth}

The results presented in this section cover experiments from experimental group 5. In these experiments a medium 2 composed of agar, with a diameter of $\mathrm{d}_{2}=1.9 \mathrm{~cm}$ was placed within saline at a lateral position of $11.6 \mathrm{~cm}$. Medium 2 possessed one of five different contrast values ranging from $\kappa_{21}=.1 .7$ to 15.2. Data are acquired for 6 different depth positions of medium 2 ranging from $\mathrm{y}_{2}=2.4$ to11.6 $\mathrm{cm}$. The temperature of the media 1 and 2 (saline bath and agar) is maintained at $21^{\circ} \mathrm{C}$. 


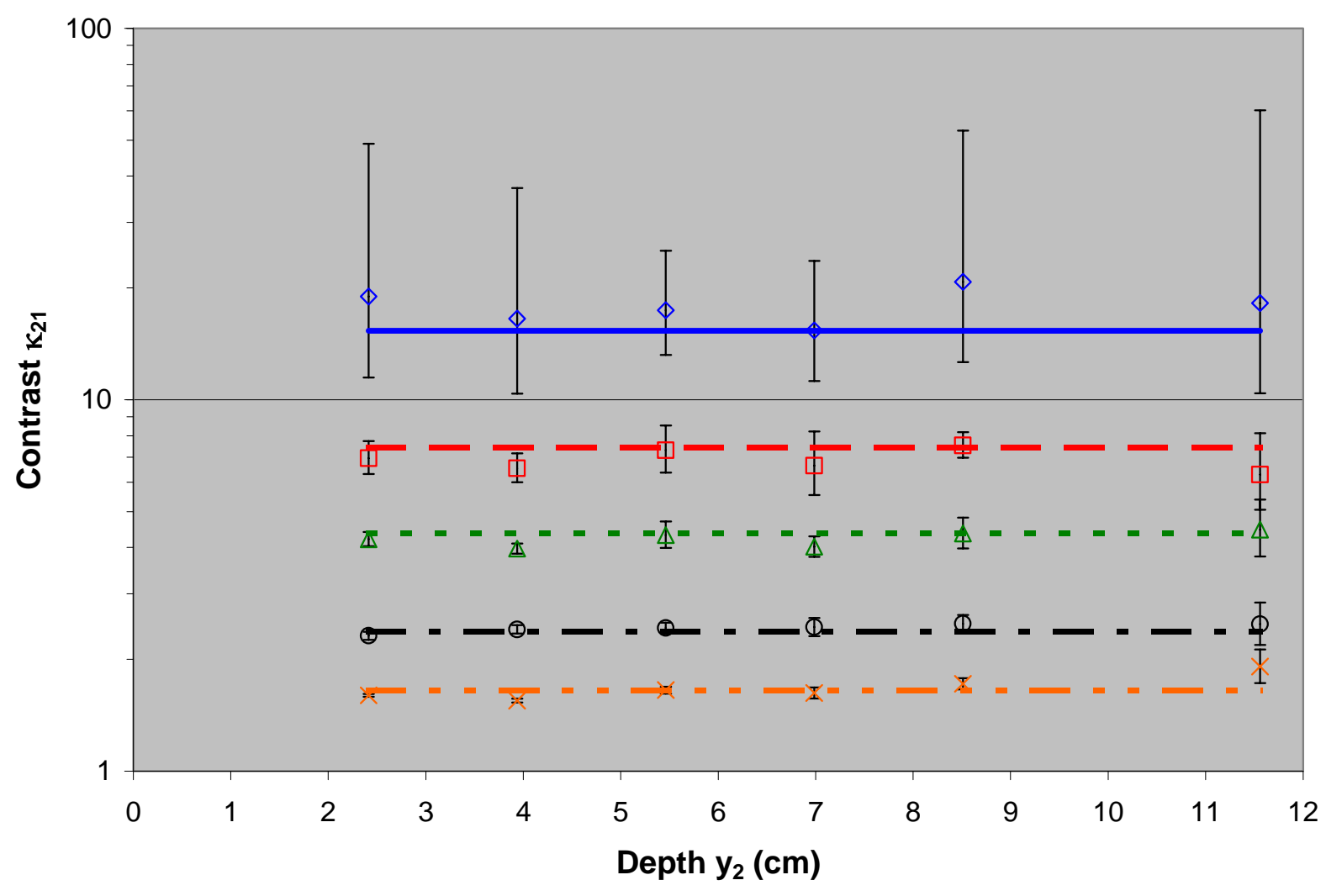

\begin{tabular}{|ccc|}
\hline $\begin{array}{c}\text { Contrast } \\
\text { Group }\end{array}$ & $\begin{array}{c}\text { Predicted } \\
\text { Contrast }\end{array}$ & $\begin{array}{c}\text { Independently } \\
\text { Measured } \\
\text { Contrast }\end{array}$ \\
\hline 15.2 & $\boldsymbol{0}$ & \\
7.4 & $\mathbf{D}$ & \\
4.4 & $\mathbf{\Delta}$ & $\mathbf{0}$ \\
2.4 & $\mathbf{O}$ & $\mathbf{-}$ \\
1.7 & $\mathbf{X}$ & \\
\hline
\end{tabular}

Figure 5-13: Predicted contrast vs. depth: The 2 media case

Predicted and 'true' (referred to as the independently measured) contrast ratios are presented for six depths of medium 2. At each depth, measurements for five different contrast ratios are collected. The predicted contrast ratios are computed from mean value of 50 separate measurements and the uncertainty bars are \pm 1 standard deviation of the mean. The 'true' contrast for each sample was determined using four lead measurement technique.

Figure 5-13 presents the predicted results as the depth of medium 2 is varied. The $\mathrm{x}$-axis of represents the depth of the medium 2 while the y-axis represents contrast ratio values. The 
results are presented in groups (families) of data points. The groupings are based on their common contrast values. The predicted contrast values are presented as individual data points along with their corresponding error bars. The value of these points is averaged from 50 separate estimates (predictions) of the agar contrast. The error bars are uniformly \pm 1 standard deviation of the average contrast value. All data points sharing the same contrast group are represented by the same symbol. The ‘true' contrast values are presented as continuous lines possessing various line styles. These values are computed by independent measurement using a standard 4 lead measurement method and are simply referred to as the independent measurement (IM) results [18].

In general, the predicted contrast results are grouped with their corresponding 'true' contrast results. Predicted results typically fall within one standard deviation of the independent measurement. The uncertainty in the predicted contrast values (error bars) increases with increasing contrast. Recalling the discussion from section 5.1.4, the coefficient of variance (c.v.) is relatively constant for all scaling factor values. The c.v. was generally between 3 and 7\%, or $5 \%$ on average.

To understand the impact of the scaling factor uncertainty on the computation of the contrast ratio uncertainty, consider Figure 5-14. This figure illustrates how the uncertainty in the scaling factor translates into uncertainties in the contrast ratio. Figure 5-14 presents 2 of the 24 data points originally presented in Figure 5-6. The value of the lower scaling factor is $0.366 \pm 0.018$ and for the higher scaling factor 0.797 \pm 0.053 . Equation (3-49) is used to compute the contrast and the corresponding uncertainties. The lower scaling factor translates into a mean contrast of 2.15 with an uncertainty of -0.084 and +0.093 . The higher scaling factor translates into a mean contrast of 8.53 with an uncertainty of -1.73 and +3.78 . The uncertainty of the lower contrast essentially balances, whereas the uncertainty of the higher contrast value is unbalanced. This imbalance is apparent in Figure 5-14. The large magnitude in the uncertainty with increasing contrast is also clearly visible. Therefore, the large uncertainties present in Figure 5-13 are consistent with findings from section 5.1.4. Thus, the larger uncertainties at higher contrast ratios are probably due to random measurement noise that affected the computation of the scaling factor. 


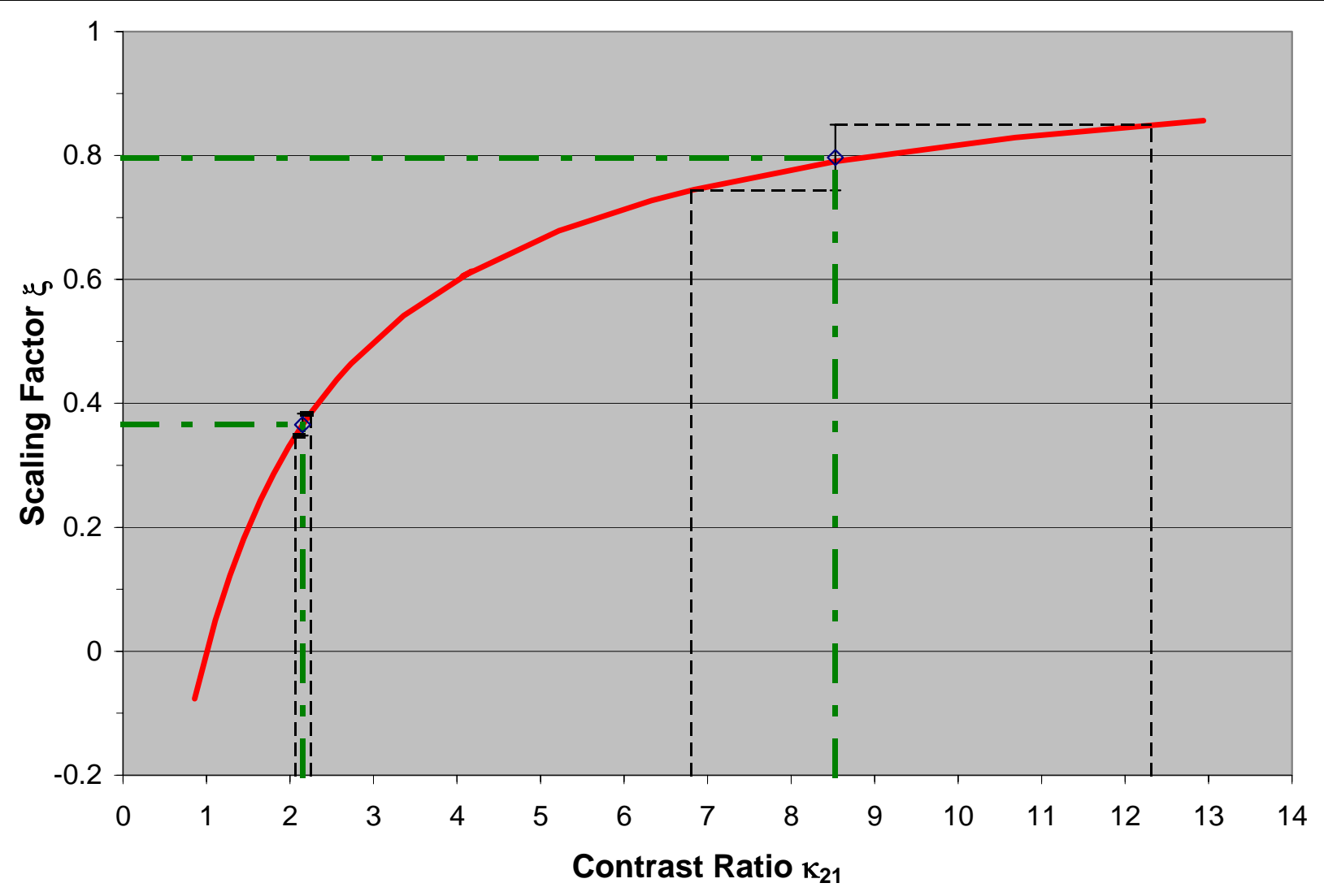

Figure 5-14: Predicted contrast uncertainties as a function of scaling factor

This figure illustrates how the uncertainty in the contrast ratio is computed from the scaling factor uncertainty. The scaling factor is the mean computed from 50 separate measurements and the uncertainty is \pm 1 standard deviation of the mean. As illustrated above, the contrast ratio is computed directly from the mean, while the uncertainty in the contrast ratio is computed from adding/subtracting the scaling factor uncertainty to the scaling factor mean.

Table 5-5 presents relative error results in a matrix representation. Relative error is given in percent and is defined in equation (5-16), as:

$$
\text { Relative Error }=\frac{\kappa_{I M}-\kappa_{\text {predicted }}}{\kappa_{I M}} \times 100 \%
$$

where $\kappa_{\mathrm{IM}}$ is the contrast from the independent measurement

$\kappa_{\text {predicted }}$ is the contrast of the predicted result

Each row of the table corresponds to the 'true' contrast and each column corresponds to the depth $\left(\mathrm{y}_{2}\right)$ of medium 2 . The intersection of a given column with a given row contains the relative error (in percent). Results are presented for 30 separate data points. In general, the error 
is less than $10 \%$. In fact, 22 of the 30 results are less than $10 \%$ while only 8 of the 30 were greater than $10 \%$. Four of the 8 cases with error greater than $10 \%$ correspond to high contrast $\left(\kappa_{21}=15.2\right)$ medium 2 . Three of the 8 cases with error greater than $10 \%$ correspond to medium 2 with great depth $\left(\mathrm{y}_{2}=11.6 \mathrm{~cm}\right)$.

Table 5-5: Error in predicted contrast for various depths: The 2 media case

\begin{tabular}{|c||c|c|c|c|c|c|}
\hline \multicolumn{1}{|c|}{$\begin{array}{c}\text { Contrast } \\
\kappa_{21}\end{array}$} & \multicolumn{7}{|c|}{ Depth $\mathrm{y}_{2}(\mathrm{~cm})$} \\
\hline 1.7 & 3.3 & 3.9 & 5.5 & 7.0 & 8.5 & 11.6 \\
\hline 2.4 & 2.6 & 1.4 & 0.0 & 1.7 & 3.8 & 13.6 \\
\hline 4.4 & 3.5 & 9.7 & 0.2 & 2.8 & 4.8 & 4.5 \\
\hline 7.4 & 6.9 & 13.8 & 1.8 & 11.9 & 1.3 & 18.4 \\
\hline 15.2 & 19.0 & 7.2 & 11.9 & 0.1 & 26.1 & 15.8 \\
\hline
\end{tabular}

Note: Error values are given in \%

Other researchers have arrived at comparable results. Glidwell and Ng [141] reported results from simulation studies that used a priori anatomical information. The purpose of the research was to improve the prediction of anisotropic tissues, in particular muscle. They performed simulation studies. Their studies attempted to estimate the heart conductivity of a simulated mongrel dog that they arbitrarily stipulated to be $0.232 \mathrm{~S} / \mathrm{m}$. The studies introduced simulated measurement noise. The simulation studies introduce $0 \%, 5 \%, 10 \%$ and $15 \%$ noise into the simulation and produced conductivities with $-4.7 \%, 7.3 \%,-12.9 \%$ and $-16.8 \%$ error respectively.

Baysal and Eyuboglu [144] reported results from experimental studies that used a priori anatomical information. They compared two different estimation methods, MiMSEE (minimum mean squares error estimator) and LSEE (least squares error estimator). Their experiment was performed on an analog circuit composed of resistive elements arranged in a 34 by 34 matrix. The circuit board was arranged as a cross section of the thorax. The region is divided into the following tissue groups: lung, heart, aorta, vertebra and sternum. The comparison with the heart in this model more closely compares with the agar in the current research since the heart is located within the domain and surrounded by the other tissues. Baysal reports his results an error of $26.9 \%$ and $6.99 \%$ using the LSEE and MiMSEE methods respectively. 
Table 5-6: Error in predicted contrast for various sizes: The 2 media case

\begin{tabular}{|c|c|c|c|c|}
\hline \multirow{2}{*}{$\begin{array}{c}\text { Contrast } \\
\kappa_{21}\end{array}$} & \multicolumn{4}{|c|}{ Size $\mathrm{d}_{2}(\mathrm{~cm})$} \\
\hline & 1.0 & 1.3 & 1.6 & 1.9 \\
\hline 1.6 & 1.4 & 9.2 & 1.3 & 1.8 \\
\hline 2.3 & 4.2 & 4.0 & 1.5 & 0.3 \\
\hline 4.2 & 5.4 & 3.5 & 7.8 & 1.1 \\
\hline 6.9 & 4.3 & 10.2 & 13.1 & 1.1 \\
\hline 13.1 & 18.4 & 2.3 & 28.0 & 25.9 \\
\hline
\end{tabular}

Note: Error values are given in \%

\subsubsection{Two Media Results: Predicted Contrast vs. Size}

Figure 5-15, presents the predicted contrast results as the size of medium 2 is varied (experimental group 6 results). In these experiments medium 2 is placed at a lateral position of $\mathrm{x}_{2}=11.6 \mathrm{~cm}$ and a depth of $\mathrm{y}_{2}=2.4 \mathrm{~cm}$. Data are acquired for 4 different depths of medium 2 ranging from $\mathrm{d}_{2}=1.0$ to1.9 $\mathrm{cm}$. Medium 2 was also varied in contrast values ranging from $\kappa_{21}=$. 1.6 to 13.2. The temperature of media 1 and 2 are maintained at $21^{\circ} \mathrm{C}$ ambient temperature. The layout and presentation of the results is nearly identical to Figure 5-12 except the $\mathrm{x}$-axis of Figure 5-15 represents the diameter of medium 2.

Table 5-6 presents error results in a matrix representation. Again, the presentation of this table is nearly identical to Table 5-5 except the columns correspond to the size of medium 2. Results are presented for 20 separate data points. The relative error is generally less than $10 \%$ with14 of the 20 results are less than $10 \%$ and 6 of the 20 were greater than $10 \%$. The 6 cases with error greater than $10 \%$ correspond to media with high contrast ( $\kappa_{21}=6.9$ and 13.1$)$. As previously observed, this is not unexpected and it's probably due to random measurement noise that affected the computation of the scaling factor, which at higher contrasts produces larger errors. 


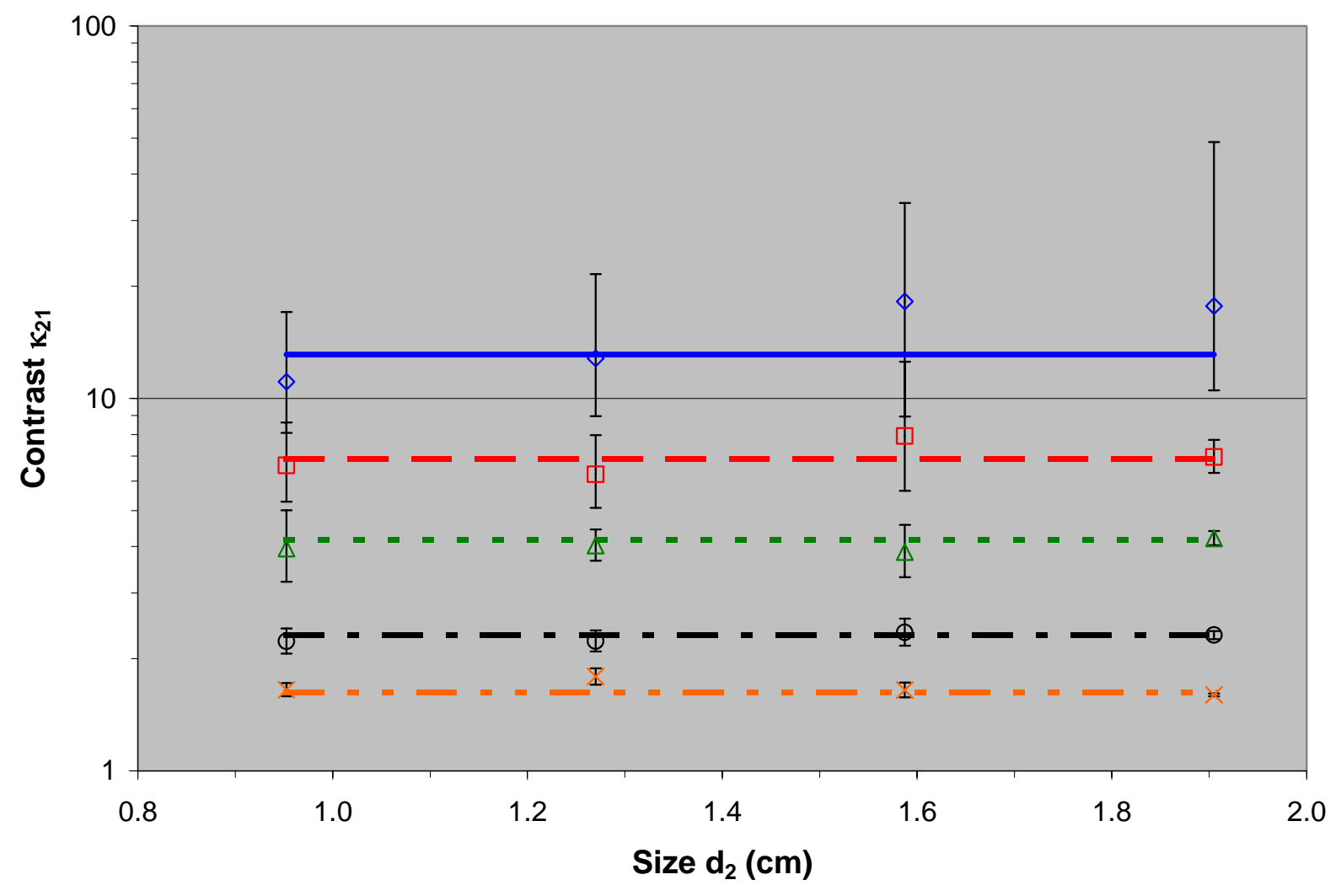

\begin{tabular}{|c|c|c|}
\hline $\begin{array}{l}\text { Contrast } \\
\text { Group }\end{array}$ & $\begin{array}{c}\text { Predicted } \\
\text { Contrast }\end{array}$ & $\begin{array}{c}\text { Independently } \\
\text { Measured } \\
\text { Contrast } \\
\end{array}$ \\
\hline 13.1 & $\nabla$ & \\
\hline 6.9 & $\square$ & - \\
\hline 4.2 & $\Delta$ & 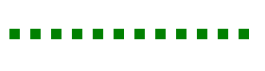 \\
\hline 2.3 & 0 & $=\cdot \square$ \\
\hline 1.6 & $x$ & {$[.=$} \\
\hline
\end{tabular}

Figure 5-15: Predicted contrast vs. size: The 2 media case

Predicted and 'true' (referred to as the independently measured) contrast ratios are presented for four sizes (diameter) of medium 2. Four each size, measurements for five different contrast ratios are collected. The predicted contrast ratios are computed from mean value of 50 separate measurements and the uncertainty bars are \pm 1 standard deviation of the mean. The 'true' contrast for each sample was determined using four lead measurement technique. 


\subsubsection{Three Media Results Using Agar}

The results presented in this section cover experiments using three media. These experiments are described in experimental groups 7 and 8 and are presented in Figures 5-16 and 5-17. Medium 1 is a saline bath with a conductivity $\sigma_{1}=0.08 \mathrm{~S} / \mathrm{m}$. Medium 2, in both experimental groups, is composed of an agar disk possessing a conductivity of $\sigma_{2}=0.1417 \mathrm{~S} / \mathrm{m}$ with a diameter $\mathrm{d}=20.3 \mathrm{~cm}$, positioned at $\mathrm{x}_{2}=11.6 \mathrm{~cm}$ and $\mathrm{y}_{2}=11.4 \mathrm{~cm}$. Medium 3, in both experimental groups, possesses four different contrast values ranging from $\kappa_{32}=1.4$ to 11.7 . Medium 3's lateral position is $\mathrm{x}_{3}=11.6 \mathrm{~cm}$ and is embedded in medium 2. The temperature of media 1,2 , and 3 is maintained at $21^{\circ} \mathrm{C}$.

Figure 5-16 presents contrast predictions of medium 3 as a function of depth position, $\mathrm{y}_{3}$, vs. contrast, $\kappa_{32}$. Figure 5-17 presents contrast prediction as a function of size, $\mathrm{d}_{3}$, vs. contrast, $\kappa_{32}$. Prediction results are presented in groups (families) of data points based on their common contrast values. In both figures, predicted contrast values are represented as individual mean data points along with their corresponding error bars. Contrast values are averaged from 49 separate computed predictions of the medium 3 and the error bars are uniformly \pm 1 standard deviation of the average contrast value. Contrast predictions with the same contrast (as determined by independent measurement) share the same symbol, while the 'true' contrast values are presented as lines with unique styles.

In general, the predicted results typically fall within one standard deviation of the independent measurement. The error in these results is presented in tables 5-7 and 5-8 in a matrix representation. Each row of the table corresponds to the 'true' contrast of medium 3. Each column of Table 5-7 corresponds to the depth of medium 3 (corresponding to results from Figure 5-16) while in Table 5-8 each column corresponds to the size of medium 3 (corresponding to results from Figure 5-17). The relative errors are quoted in percent.

Two trends are apparent in Table 5-7. First, the relative error increases with increasing contrast. Second, the relative error increases with increasing depth. Both trends are evident in the 2 media experiments. The reason for the first trend is the inverse method tends to produce higher contrast uncertainties at higher contrast values. The second trend is caused by the decrease in signal strength as medium 3 moves deeper into the data acquisition tank. Assuming 
the random measurement noise is unchanged by the location of medium 3 , the relative error should increase as the current pattern's magnitude decreases.

Two trends are apparent in Table 5-8. As in the case with Table 5-7, the first trend is an increase in relative error with increasing contrast, which is due to production of higher contrast uncertainties at higher contrast values. The second trend is that the relative error increases with decreasing size. By the same reasoning as presented above, assuming the random measurement noise is unchanged by the size of medium 3 , the relative error should increase as the current pattern's magnitude decreases.

Table 5-7: Error in predicted contrast for various depths: The 3 media case

\begin{tabular}{|c||c|c|c|c|}
\hline \multicolumn{1}{|c|}{ Contrast } & \multicolumn{4}{|c|}{ Depth $\mathrm{y}_{3}(\mathrm{~cm})$} \\
\cline { 2 - 5 } $\mathrm{k} 32$ & 2.8 & 4.3 & 5.8 & 7.4 \\
\hline 1.4 & 0.3 & 1.1 & 3.5 & 1.1 \\
\hline 2.7 & 3.1 & 6.7 & 1.1 & 1.6 \\
\hline 4.9 & 6.9 & 5.6 & 24.1 & 20.2 \\
\hline 11.7 & 16.9 & 1.5 & 33.2 & 0.2 \\
\hline
\end{tabular}

Note: Error values are given in \%

Table 5-8: Error in predicted contrast for various sizes: The 3 media case

\begin{tabular}{|c||c|c|c|c|}
\hline \multicolumn{1}{|c||}{ Contrast } & \multicolumn{4}{c|}{ Size $\mathrm{d}_{3}(\mathrm{~cm})$} \\
\cline { 2 - 5 }$\kappa_{32}$ & 1.0 & 1.3 & 1.6 & 1.9 \\
\hline \hline 1.4 & 5.7 & 1.5 & 1.4 & 0.5 \\
\hline 2.8 & 3.0 & 1.2 & 3.2 & 6.3 \\
\hline 4.9 & 5.1 & 5.0 & 4.4 & 10.7 \\
\hline 11.7 & 23.2 & 17.2 & 1.5 & 2.8 \\
\hline
\end{tabular}

Note: Error values are given in \% 


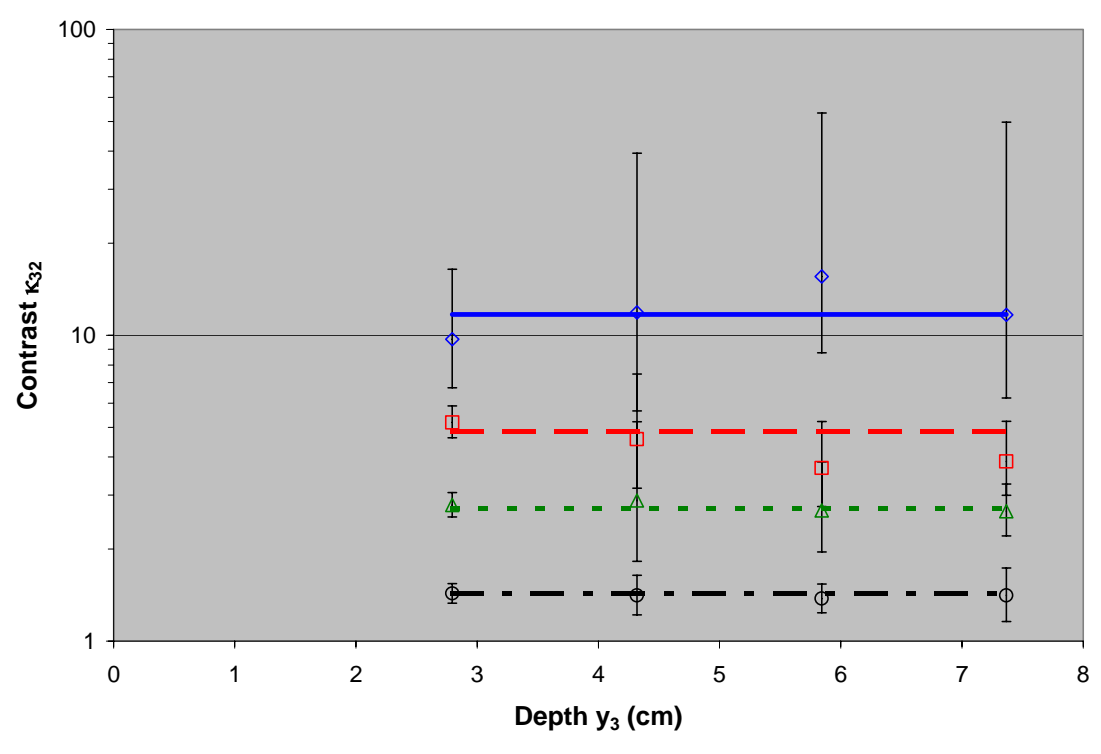

\begin{tabular}{|ccc|}
\hline $\begin{array}{c}\text { Contrast } \\
\text { Group }\end{array}$ & $\begin{array}{c}\text { Predicted } \\
\text { Contrast }\end{array}$ & $\begin{array}{c}\text { Independently } \\
\text { Measured } \\
\text { Contrast }\end{array}$ \\
\hline 11.7 & $\mathbf{0}$ & \\
4.9 & $\mathbf{0}$ & \\
2.7 & $\mathbf{0}$ & \\
1.4 & $\mathbf{0}$ & \\
\hline
\end{tabular}

Figure 5-16: Predicted contrast vs. depth: The 3 media case

Predicted and 'true' (referred to as the independently measured) contrast ratios are presented for four depths of medium 3. At each depth, measurements for four different contrast ratios are collected. The predicted contrast ratios are computed from mean value of 50 separate measurements and the uncertainty bars are \pm 1 standard deviation of the mean. The 'true' contrast for each sample was determined using four lead measurement technique.

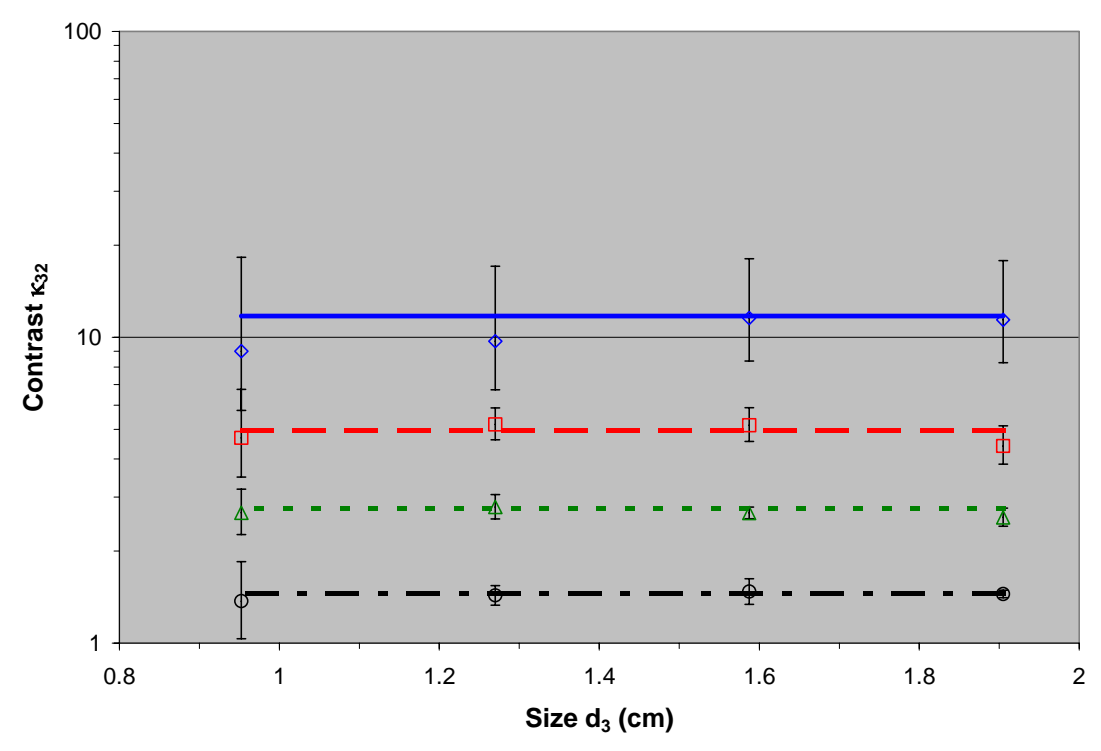

\begin{tabular}{|ccc|}
\hline $\begin{array}{c}\text { Contrast } \\
\text { Group }\end{array}$ & $\begin{array}{c}\text { Predicted } \\
\text { Contrast }\end{array}$ & $\begin{array}{c}\text { Independently } \\
\text { Measured } \\
\text { Contrast }\end{array}$ \\
\hline 11.7 & $\mathbf{0}$ & \\
4.9 & $\mathbf{0}$ & \\
2.8 & $\mathbf{0}$ & \\
1.4 & $\mathbf{0}$ & \\
\hline
\end{tabular}

Figure 5-17: Predicted contrast vs. size: The 3 media case

Predicted and 'true' (referred to as the independently measured) contrast ratios are presented for four sizes (diameter) of medium 3. Four each size, measurements for four different contrast ratios are collected. The predicted contrast ratios are computed from mean value of 50 separate measurements and the uncertainty bars are \pm 1 standard deviation of the mean. The 'true' contrast for each sample was determined using four lead measurement technique. 


\subsubsection{Three Media Results Using Liver and Oatmeal}

The results presented in this third section cover experiments using three media, but with the difference that medium 2 is composed of calves liver and medium 3 is composed of an oatmeal/saline solution mixture. This experiment is described in experimental groups 9. Medium 1 is a saline bath with a conductivity $\sigma_{1}=0.09 \mathrm{~S} / \mathrm{m}$. Medium 2 is a freshly slaughtered calf's liver disk with a diameter $\mathrm{d}=20.3 \mathrm{~cm}$, positioned at $\mathrm{x}_{2}=11.6 \mathrm{~cm}$ and $\mathrm{y}_{2}=11.4 \mathrm{~cm}$ possessing a conductivity that ranges from $\sigma_{2}=0.11$ to $0.14 \mathrm{~S} / \mathrm{m}$. Medium 3 is composed of an oatmeal-saline mixture that is positioned at $x_{3}=11.6$ and $y_{3}=2.8 \mathrm{~cm}$, has a size of $d_{3}=1.9 \mathrm{~cm}$ and possesses a single conductivity of $\sigma_{3}=0.25 \mathrm{~S} / \mathrm{m}$. The temperature of media 1,2 , and 3 ranges from 18 to $19{ }^{\circ} \mathrm{C}$ throughout the 17 hour data collection session.

Compared with the agar samples, which are predominantly conductive in nature, the liver media possesses complex electrical properties, conductivity and relative permittivity. The purpose of the experiments is to determine how accurately the EPET method predicts the electrical properties of medium 2 and 3. The indirect reconstruction method (using finite difference and Newton-Raphson methods for forward and inverse solutions) is applied this case.

\subsubsection{Liver Results}

Results in figures 5-18 and 5-19 present predictions of the conductivity and relative permittivity ( $\sigma_{2}$ and $\varepsilon_{\mathrm{r} 2}$ ) of medium 2 (liver) vs. time respectively. The x-axis in both figures represents the time that has passed since the first independent measurement of electrical properties is acquired. The y-axis of Figure 5-18 represents conductivity values in S/m while the y-axis for Figure 5-19 represents the relative permittivity. The predicted conductivity/relative permittivity values are presented as individual data points (diamonds). The value of these points is averaged from 50 separate predictions. The ‘true’ conductivity/relative permittivity values are presented as interpolations stretching between the first and last independent measurements of the liver electrical properties acquired at 0 hours and 17 hours elapsed time using four lead measurement, which are represented with x-symbols. The interpolated conductivity and relative permittivity values are presented as a continuous dashed line. The linear models of these independent measurements are presented in equations (5-17) and (5-18) for conductivity and relative permittivity respectively. These values were computed by independent measurement using a standard 4 lead measurement method. 


$$
\begin{gathered}
\sigma_{2}^{\mathrm{IM}}=0.002 \cdot \text { time }+0.112[\mathrm{~S} / \mathrm{m}] \\
\varepsilon_{r 2}^{\mathrm{IM}}=-279 \cdot \text { time }+14190
\end{gathered}
$$

The standard error of the estimate [156] between the EPET predicted and independently measured conductivity and relative permittivity values is presented in Table 5-9. The error for the liver conductivity (1.9\%) over the 17 hours is consistent with the agar results (between 0.5 and $6.3 \%$ ) for the same position, size and contrast range. The error for the liver relative permittivity is slightly higher (5.1\%), but is still within the range of the conductivity results for the 3 media agar experiments.

Table 5-9: Relative Standard Error of the Estimate for Liver and Oatmeal

\begin{tabular}{|c||c|}
\hline \multicolumn{1}{|c||}{ Case } & $\begin{array}{c}\text { Relative Standard } \\
\text { Error of the } \\
\text { Estimate } \pm 1 \text { Std } \\
(\%)\end{array}$ \\
\hline \hline Liver conductivity & $1.9 \pm 0.03$ \\
\hline Liver relative permittivity & $5.1 \pm 0.31$ \\
\hline $\begin{array}{c}\text { Oatmeal conductivity } \\
\text { (unadjusted phase) }\end{array}$ & $17.4 \pm 2.18$ \\
\hline $\begin{array}{c}\text { Liver conductivity } \\
\text { (phase adjusted) }\end{array}$ & $6.7 \pm 0.88$ \\
\hline
\end{tabular}

The results display a very obvious time variation in the liver's conductivity and relative permittivity values. Over the 17 hour time span, the conductivity rises about $24 \%$ in value while the relative permittivity declines about $32 \%$. The variation in conductivity and relative permittivity over time is an expected result. A number of studies performed by other researchers are available for comparison. Kehrer et al [158] report changes in the impedance of liver over the first few hours after slaughter. He noted that very quickly after death the alpha dispersion (low frequency) disappears rapidly (within 2 to 3 hours). He theorizes this was due to the closing of the gap junctions between cells within the first few minutes to a couple of hours. There is a clear trend change in the electrical properties measured in this dissertation work, however the cause is probably due to factors other than the closing of gap junctions.

In addition to Kehrer's results, the liver tissue is decaying. Cell membranes are steadily dissolving. Recalling the impedance model presented in chapter 2, the tissue relative 
permittivity is theorized to be predominantly due to the presence of the cellular membranes. As these membranes dissolve, the source of tissue relative permittivity also disappears.

Additionally, as the cell membranes dissolve, the intra- and extracellular fluids combine. Since the intracellular fluid possesses a higher conductivity than the extracellular fluid, and observer would expect the overall conductivity to increase as the cell membranes dissolve. This also provides a large current path through the tissue. 


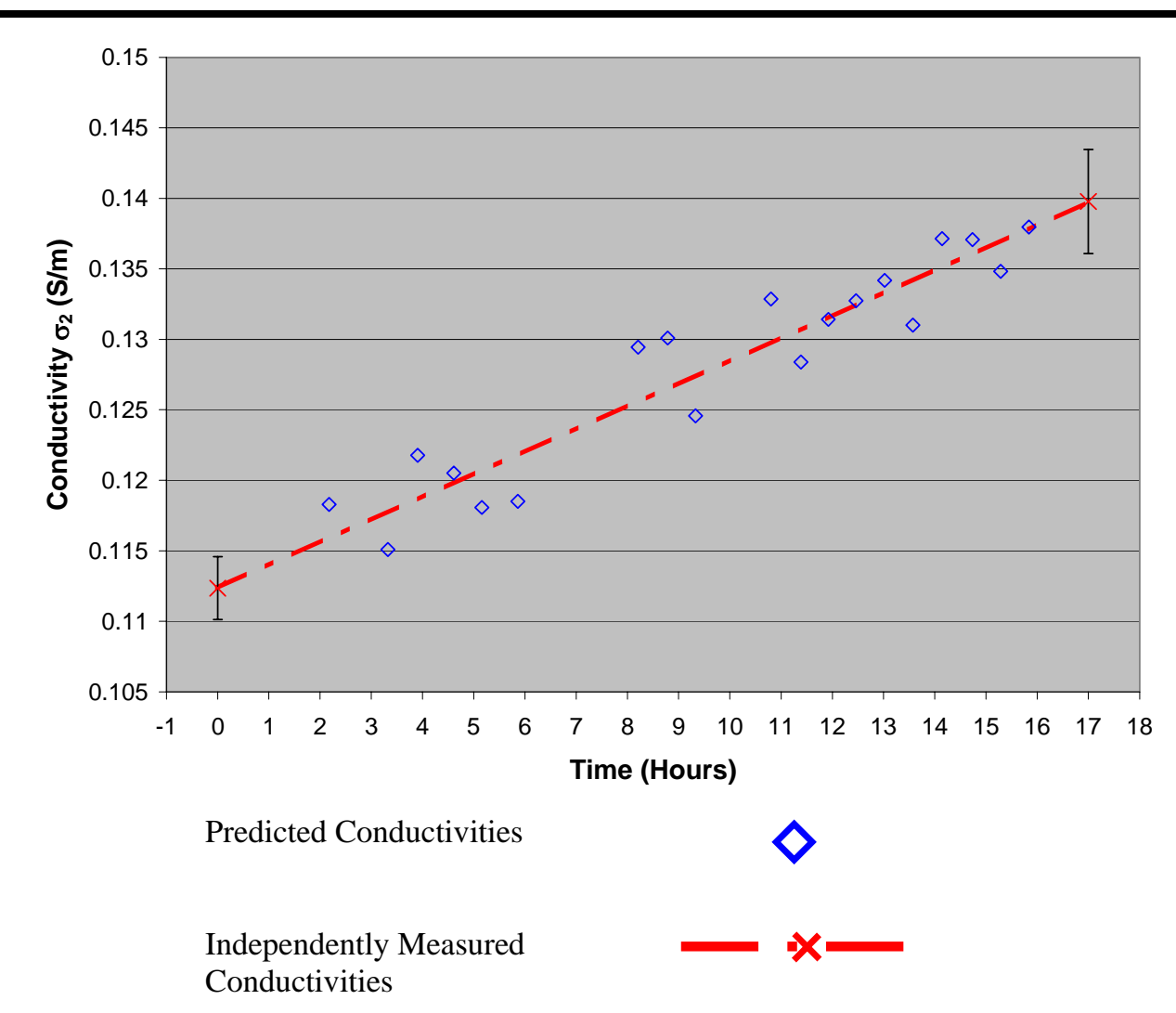

Figure 5-18: Predicted liver conductivity vs. time

Predicted and 'true' (referred to as the independently measured)

conductivity are presented for liver (medium 2). The predicted

conductivities are computed from mean value of 50 separate

measurements and the uncertainty bars are \pm 1 standard deviation of the

mean. The 'true' conductivity for each sample was determined using four

lead measurement technique before and following the experiment. The

expected 'true' conductivity for the period during the experiment was

computed by interpolating between these measurements.

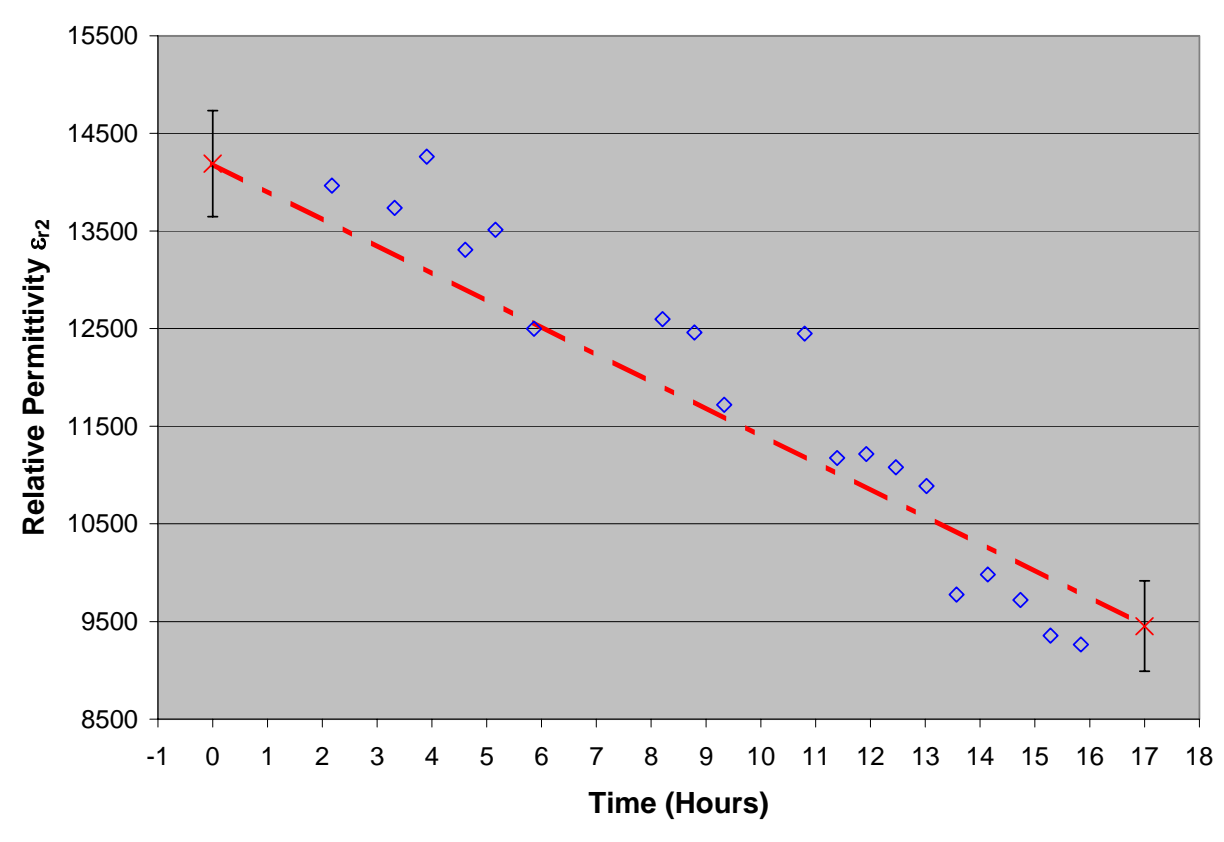

Predicted Relative

Permittivities

Independently Measured

Relative Permittivities

\section{Figure 5-19: Predicted liver relative permittivity vs. time}

Predicted and 'true' (referred to as the independently measured) relative permittivity are presented for liver (medium 2). The predicted relative permittivities are computed from mean value of 50 separate measurements and the uncertainty bars are \pm 1 standard deviation of the mean. The 'true' relative permittivity for each sample was determined using four lead measurement technique before and following the experiment. The expected 'true' relative permittivity for the period during the experiment was computed by interpolating between these measurements. 


\subsubsection{Oatmeal Results}

The oatmeal conductivity results are presented in Figure 5-20. The x-axis represents the time that has passed since the first independent measurement of electrical properties was acquired. The y-axis of Figure 5-20 represents conductivity values in S/m. The predicted conductivity values are presented as individual data points (diamonds). Note, the conductivity chart has a second set of predicted results denoted as the phase adjusted results that are represented as triangles. Each data point is averaged from 50 separate conductivity predictions for the oatmeal. The 'true' conductivity values are presented as a continuous dashed red line constant over time and were computed by independent measurement using a standard 4 lead measurement method.

The conductivity results appear to consistently predict values less than the expected oatmeal conductivity. The error for the oatmeal conductivity (Table 5-9) over the 17 hours is consistently greater (on average 17.4\%) than agar results predicted for similar position and size (between 0.5 and 5.8\%). The oatmeal relative permittivity over the same time period is negative and large (approximately -20,000). The relative permittivity results are not presented in a chart since negative permittivities are undefined and such results hold little meaning for physical interpretation. However, negative permittivity predictions suggest the source of a problem that can explain the high error in the conductivity results. Negative permittivity predictions are likely due to a phase error in the measured current pattern data. A negative relative permittivity could be explained by the 'positive' phase angle versus a 'negative' phase angle in the current data. Subsequent to the data collection session, the experimental setup was recalibrated. A phase-shift of 16 degrees was observed using agar and metallic samples as test objects. When purely conductive media are under test, no phase shift is expected. Candidates for the source of this phase shift are:

1. The driver/sensor electronics

2. the Solartron 1260 phase/gain amplifier, or

3. interface issues (i.e. the electrode/electrolyte electrochemical reactions).

Further testing was unable to narrow the source of the phase shift. 


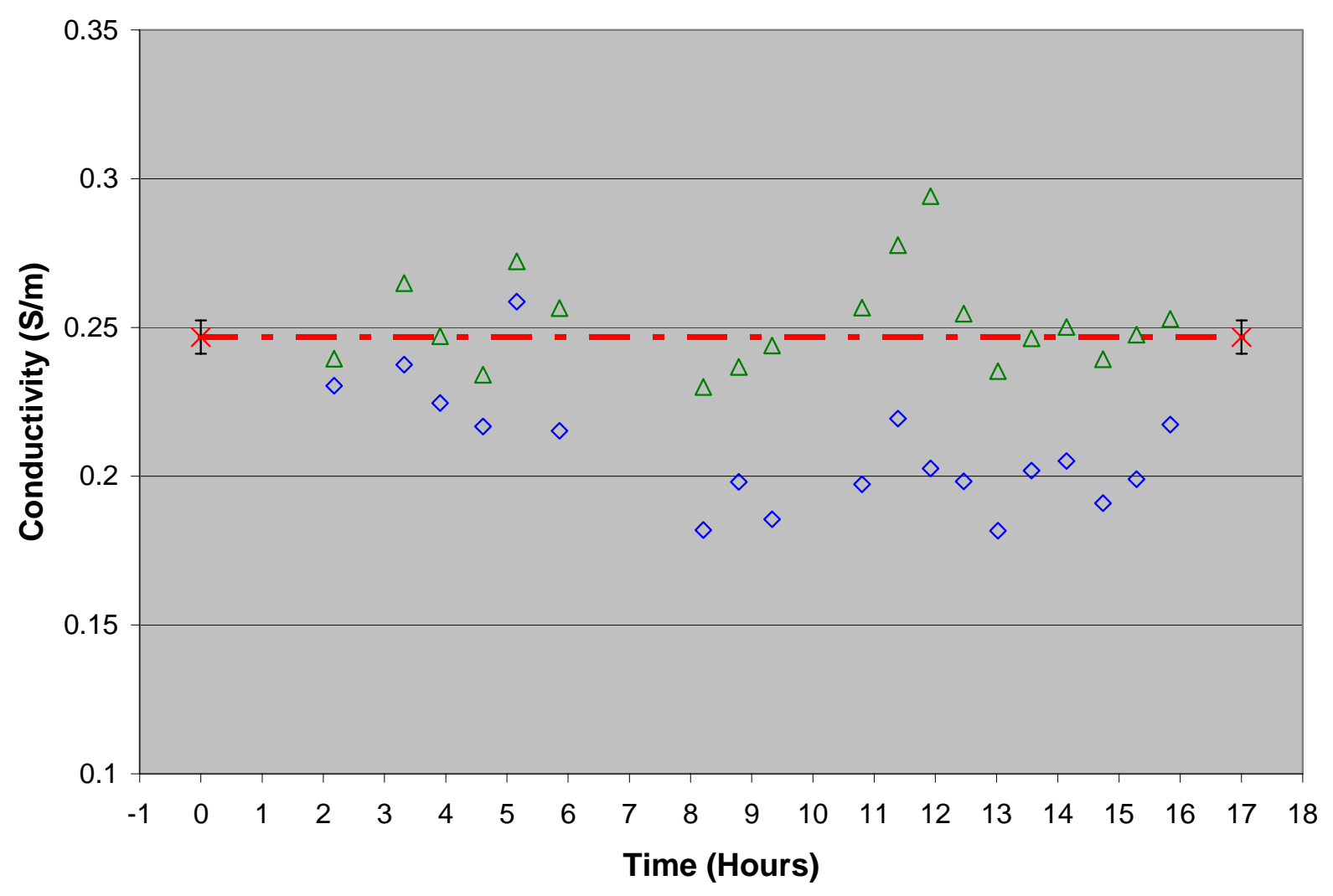

Predicted Conductivities (no phase adjustment)

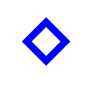
Independently Measured Conductivities

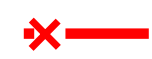

Predicted Conductivities

(with phase adjustment)

Figure 5-20: Predicted oatmeal conductivity vs. time

Predicted and 'true' (referred to as the independently measured) conductivity are presented for the oatmeal/saline mixture (medium 3). The predicted conductivities are computed from mean value of 50 separate measurements and the uncertainty bars are \pm 1 standard deviation of the mean. The 'true' conductivity for each sample was determined using four lead measurement technique before and following the experiment. The expected 'true' conductivity for the period during the experiment was computed by interpolating between these measurements.

To test the notion that the negative relative permittivity and under predicted conductivity are due to the phase error, the predicted conductivity and relative permittivity are phase shifted to match the expected phase angle. For a $0.25 \mathrm{~S} / \mathrm{m}$ saline solution the relative permittivity is approximately 80. The expected phase angle is (defined in equation (5-19)): 


$$
\text { phase angle }=\tan ^{-1}\left(\frac{\omega \varepsilon_{0} \varepsilon_{r}}{\sigma}\right)=0.05^{\circ}
$$

This angle is very small, thus the phase corrected conductivity is approximately the absolute magnitude of the complex conductivity (defined in equation (5-20)).

$$
\sigma_{\text {pha sec orrected }}=\sqrt{\sigma_{\text {uncorrected }}^{2}+\left(\omega \varepsilon_{0} \varepsilon_{r}\right)^{2}}
$$

The phase adjusted conductivity prediction is overlaid (triangle) with the uncorrected conductivity (diamond). These values reduce the error from $17.4 \%$ to $6.7 \%$, which is in line with agar results. With this phase correction, it appears that the oatmeal results are reasonable.

\subsubsection{Understanding the liver/oatmeal results}

The time varying problems are a significant source of uncertainty and error in these experiments. Three types of time varying sources are:

1. changing water conductivity,

2. changing liver properties and

3 . changing temperature.

Changing water conductivity results from the contamination of the blood in the liver mixing with the saline bath (medium 1). Blood has a conductivity of $0.7 \mathrm{~S} / \mathrm{m}$ at $25{ }^{\circ} \mathrm{C}$ [15]. When mixed with a saline solution of approximately $0.09 \mathrm{~S} / \mathrm{m}$, the saline solution's conductivity rises. Changing liver properties is most likely caused by the liver decaying. This change has already been discussed. The liver is allowed to reach room temperature and remains at room temperature $\left(17\right.$ to $\left.19^{\circ} \mathrm{C}\right)$ throughout the experiments.

The choice to use liver is balanced between advantages and drawbacks. The advantage is that liver possessed relative permittivity properties in the neighborhood found in living tissue at $50 \mathrm{kHz}$. It is isotropic in its electrical properties unlike other bulk tissue, for example muscle is highly anisotropic. It was not possible to reproduce an artificial phantom with similar characteristics. The disadvantage is that blood in the liver mixed with the saline resulting in changing conductivity values. The changing saline conductivity values were estimated statistically by examining the single medium (uniform case) current reading before and after each experimental run and comparing these readings with independently measured bath water conductivity measurements (using a handheld conductivity meter). Even though these estimates 
produced reasonable results as shown above, the method was tedious and susceptible to erroneous fluctuations.

A typical experiment took about 60 minutes to complete (including preparation and cleanup) and the data collection session lasted nearly 17 hours. Aside from the saline conductivity changes, the liver properties also changed. Over the 17 hours that the experiment lasted, the conductivity and relative permittivity changed from $1 \%$ to $2 \%$ per hour. It was desirable to complete the actual measurement of the liver as quickly as possible.

\subsection{Charge-charge Correlation vs. Indirect Reconstruction Method}

The final section of this chapter compares the two numerical methods used in the Electrical Property Enhanced Tomography (EPET) to predict electrical property values. These methods, the charge-charge correlation method (CCCM) and the indirect reconstruction method (IRM), are described in detail in chapters 3 and 4. The CCCM uses a high-contrast forward solution (equation (3-45)) to produce a predicted current pattern. From this predicted current pattern a scaling factor, $\xi$, is computed (equation (3-47)) which is used to compute the predicted contrast ratio, $\kappa$ (equation (3-49)). The IRM uses predictions of the electrical properties in the finite difference method (FDM) to compute the predicted current patterns. The Newton-Raphson method is then used to adjust the predicted electrical properties until they approach the measured current patterns (i.e. the least squares error estimate is minimized). The CCCM is employed to predict contrast ratios used in all experimental groups except experimental group 9, in which the IRM was employed.

These two methods are now compared side by side using data from experimental group 5 (the two media case). In these experiments a medium 2 composed of agar, with a diameter of $\mathrm{d}_{2}=1.9 \mathrm{~cm}$ was placed at a lateral position of $11.6 \mathrm{~cm}$. Medium 2 consisted of five different contrast values ranging from $\kappa_{21}=1.7$ to 15.2. Data are acquired for 6 different depth positions of medium 2 ranging from $y_{2}=2.4$ to $11.6 \mathrm{~cm}$. The temperature of the media 1 and 2 (saline bath and agar) was maintained at $21^{\circ} \mathrm{C}$ (ambient temperature).

The predicted contrast ratios are presented in Table 5-10. The relative errors and computation times for both numerical methods are presented in Table 5-11. The relative errors of both the IRM and CCCM are comparable at low contrast $\left(\kappa_{21}=1.7\right.$ and 2.4$)$ but begin to diverge as the contrast of medium 2 increases. The error using the CCCM remains under 10\% 
until the highest contrast ratio $\left(\kappa_{21}=15.2\right)$ and increases less rapidly than the IRM. The error using the IRM, by contrast, rises above $10 \%$ when the contrast ratio of medium 2 is relatively low $\left(\kappa_{21}=4.4\right)$. In the two media case, the CCCM produces better predictions of the contrast ratio at moderate contrast values $\left(4.4<\kappa_{21}<15.2\right)$ than the IRM.

The computation time for the IRM is between 3 and 4 minutes. The time depends somewhat on the magnitude of the contrast ratio for medium 2 and increases as the contrast ratio increases. By contrast, the CCCM performs the prediction computations in a fraction of a second, a more than 10,000 fold improvement over the IRM. The computation time is fairly constant and is independent of the contrast ratio. The comparison shows that the CCCM produces predictions of internal electrical properties that are at least equal to, and as contrast increases, superior to, the IRM. Furthermore, the CCCM achieves these results in a fraction of the time needed by the IRM.

Table 5-10: Predicted Contrast Results using IRM and CCCM

\begin{tabular}{|c||c|c|}
\hline \multicolumn{1}{|c||}{$\begin{array}{c}\text { Independently } \\
\text { Measured } \\
\text { Contrast } \\
\text { Ratio } \\
\kappa_{21}\end{array}$} & \multicolumn{2}{c|}{$\begin{array}{c}\text { Predicted Contrast Ratio } \\
( \pm 1 \text { standard deviation })\end{array}$} \\
\cline { 2 - 3 } & IRM & CCCM \\
\hline 1.7 & $1.63 \pm 0.01$ & $1.60 \pm 0.01$ \\
\hline 2.4 & $2.31 \pm 0.05$ & $2.31 \pm 0.06$ \\
\hline 4.4 & $3.93 \pm 0.15$ & $4.21 \pm 0.18$ \\
\hline 7.4 & $5.81 \pm 0.42$ & $6.96 \pm 0.65$ \\
\hline 15.2 & $11.19 \pm 3.78$ & $17.69 \pm 7.19$ \\
\hline
\end{tabular}


Table 5-11: Comparing relative errors and computation times for IRM and CCCM

\begin{tabular}{|c|c|c||c|c|}
\hline \multirow{2}{*}{$\begin{array}{c}\text { Independently } \\
\text { Measured } \\
\begin{array}{c}\text { Contrast } \\
\text { Ratio } \\
\kappa_{21}\end{array}\end{array}$} & \multicolumn{2}{|c||}{ Relative Error (\%) } & \multicolumn{2}{c|}{ Computation Time (seconds) } \\
\cline { 2 - 5 } & IRM & CCCM & IRM & CCCM \\
\hline \hline 1.7 & 4.1 & 5.9 & $190 \pm 2$ & $0.0137 \pm 0.0002$ \\
\hline 2.4 & 3.7 & 3.7 & $201 \pm 2$ & $0.0137 \pm 0.0002$ \\
\hline 4.4 & 10.7 & 4.3 & $209 \pm 3$ & $0.0138 \pm 0.0002$ \\
\hline 7.4 & 21.5 & 5.9 & $213 \pm 3$ & $0.0137 \pm 0.0002$ \\
\hline 15.2 & 26.4 & 16.4 & $222 \pm 4$ & $0.0137 \pm 0.0002$ \\
\hline
\end{tabular}




\section{SUMMARY AND FUTURE WORK}

Electrical Impedance Tomography (EIT) is a technique for determining conductivity and relative permittivity distributions within the human body. EIT has traditionally relied mainly on numerical methods to solve the non-linear Poisson's equation. Both direct (single iteration) and indirect (multiple iteration) methods are used, but indirect methods are the dominant method of choice. The forward and inverse problems are performed sequentially and iteratively until the LSEE falls below a minimum threshold. The forward solution is time consuming because it is computationally intensive. The inverse solution uses the Newton-Raphson method, which produces a matrix representation that is ill-posed. The matrix must be inverted, which produces erroneous results. The ill-posed issues are generally addressed using regularization or $a$ priori geometric information.

\subsection{The Proposed Solution}

This research proposed an alternative approach to solving the Poisson equation. This approach used the Green's theorem with Dirichlet boundary conditions to relate internal charge distribution and applied boundary potential to the internal potential field. The Green's function is determined solely by the shape of the domain.

Manipulation of the Green's theorem led to the charge-charge correlation relationship. This relationship forms the basis of the impedance reconstruction method, called Electrical Property Enhance Tomography (EPET). EPET does not attempt to create the image with the electrical data but rather adds electrical property information to the existing modality and, in fact, requires the data from another modality to locate the position of internal structures in the object. The EPET method uses a priori structural (geometric) information to identify the location of internal charge buildup. The buildup is expected to occur at the boundary between different electrical properties. Using a well defined domain, the charge-charge correlation relationship, and a priori structural information, the EPET method produces estimates of the internal electrical properties within a domain. 


\subsection{Conclusions}

First two media cases using saline for the first media and either agar or metal for the second media were considered. The observed current pattern possessed a Gaussian-like shape. Cross-correlation analysis showed that the current pattern shape was a function of the position of the second media only. Similar analysis demonstrated that the magnitude of the current pattern is a function of the size and contrast ratio. Its shape remained unchanged as the size and contrast ratio of the second medium varied, but the magnitude changed with these variations.

The magnitude of the current pattern was maximized when the second medium was metallic (i.e. when the contrast ratio was very large). As the contrast ratio decreased, the magnitude of the current pattern decreased. Analysis comparing the magnitude of the current patterns with cases where the contrast ratios ranged from approximately 1 to 15 demonstrated that the scaling factor function, $\xi$ (equation (3-48)), reasonably modeled (with a coefficient of determination $>0.9$ ) the magnitude of the current pattern. Since the scaling factor was described by a function involving only the contrast ratio and was independent of the position or size of the second media, this finding supported the assertion that the inverse solution of the EPET method could accurately estimate the unknown electrical properties of the second medium.

Next, consider the forward solution for two media cases using saline for the first medium and metal for the second medium. The forward solution was applied and compared to measured results where the lateral position, depth position, and size of the second media were varied. In all cases the coefficient of determination was at least 0.9. Consequently, the forward solution was deemed an accurate model for predicting the current patterns for all cases under study.

Analysis using the peak current as a test point demonstrated that the forward solution predicts the measured peak currents nearly perfectly (coefficient of determination $>0.99$ ) as the depth and size of the second medium was varied. This result suggested the forward solution could be used to estimate the second medium's minimum size and contrast ratio when it was placed in the center of the tank. Using an estimate of the measurement noise floor (level), a relationship between minimum contrast and minimum size was derived.

The inverse solution for both two and three media cases produced similar results. The error was less than $10 \%$ for 22 of the 30 cases in two media experiments when the depth of the second media was varied, and for 14 of the 20 cases when the diameter of the second medium 
was varied. In three medium experiments, the error was less than $10 \%$ for 10 of the 16 cases when the depth of the third media was varied and when the diameter of the third medium was varied. These results compared well with reports from other researchers. Glidwell and Ng [141] reported errors of $4.7 \%, 7.3 \%, 12.9 \%$ and $16.8 \%$ from simulation studies that introduce $0 \%, 5 \%$, $10 \%$ and 15\% noise respectively. Baysal and Eyuboglu [144] reported an error of $26.9 \%$ and 6.99\% using the LSEE and MiMSEE methods respectively.

Three trends were apparent from these error results. First, the error increased with increasing contrast; second, the error increased with increasing depth (of the second and third media in the two and three media experiments respectively); and third, the error increased with decreasing diameter (of the second and third media in the two and three media experiments respectively). Analysis of the inverse function (equation (3-49)) demonstrated that the inverse method was less accurate at higher contrast values. Analysis comparing the peak current for measured versus predicted cases demonstrated that as current pattern magnitude decreased with decreasing diameter (or increasing depth), relative uncertainty due to measurement noise increased. Given that the error results were in line with other researchers and that the detrimental effects of high contrast and small diameter were understood (as was discussed earlier), the inverse solution was deemed to be at least as reliable as the indirect reconstruction method.

The three media experiments using saline for medium 1, liver for medium 2 and an oatmeal/salt mixture for medium 3 were viewed in a more qualitative than quantitative light. Errors of the predicted liver conductivity and relative permittivity are 1.9\% and 5.1\% respectively. Over a 15 hour time span, the conductivity rose about $24 \%$ in value while the relative permittivity declined about $32 \%$. These changes over time were expected due to decay of the liver tissue. As these cell membranes dissolved, the source of tissue relative permittivity also disappeared, and conductivity increased. Additionally the intra- and extracellular fluids combined resulting in an overall conductivity increase.

The error for the oatmeal conductivity was on average $17.4 \%$. The oatmeal relative permittivity was negative, indicating a phase shift error in the measured data. Sources for an error source were identified (electronics, instrumentation, and electrode/electrolyte reactions). Correcting the phase shift reduced the conductivity error from $16 \%$ to $6.7 \%$. 
These experiments with liver were viewed as an indication of possible applications of the EPET method in the future. Liver was chosen because it possessed conductivity and relative permittivity properties that could not be reconstituted using manmade materials and methods. On the negative side, blood in the liver mixed with the saline causing the saline conductivity values to change. Also, a typical experiment required nearly 60 minutes to complete (including preparation and cleanup). Over the 15 hours that the experiment session lasted, the conductivity and relative permittivity changed from $1 \%$ to $2 \%$ per hour. It was desirable to complete the actual measurement of the liver as quickly as possible. Phase measurement errors were significant. Phase measurement were not an issue for purely conductive materials (agar and metal), but were vital when the relative permittivity was estimated.

When comparing the indirect reconstruction method (IRM) and charge-charge correlation method, the relative errors of both the IRM and CCCM were comparable at low contrast $\left(\kappa_{21}=\right.$ 1.7 and 2.4) but began to diverge as the contrast of medium 2 increased. The error using the CCCM remained under $10 \%$ until the highest contrast ratio $\left(\kappa_{21}=15.2\right)$. The error using the IRM, by contrast, rose above $10 \%$ when the contrast ratio of medium 2 is relatively low ( $\kappa_{21}=$ 4.4). In the two media case, the CCCM produced better predictions of the contrast ratio at moderate contrast values $\left(4.4<\kappa_{21}<15.2\right)$ than the IRM.

The computation time for the IRM was between 3 and 4 minutes. The time depended on the magnitude of the contrast ratio for medium 2 and increased as the contrast ratio increased. By contrast, the CCCM performed the prediction computations in a fraction of a second, a more than 10,000 fold improvement over the IRM. The computation time was fairly constant and was independent of the contrast ratio of medium 2. The comparison showed that the CCCM produced predictions of internal electrical properties that were at least equal to, and in moderate contrast ranges superior to, the IRM. Furthermore, the CCCM achieved these results in a fraction of the time needed by the IRM.

\subsection{Future Work}

The conclusions reached in this report suggest a need for further research. Proposals for future work should address some technical issues as well as theoretical issues. This proposal falls into three categories: evolving the theory, developing electronics, constructing a new data 
acquisition tank, and performing laboratory experiments. The purpose of this work would be to transition this research work to an eventual clinical application.

The work presented in this report was limited to a quasi-two dimensional application. The theory must be modified for three-dimensional applications. This effort will require solving Green's function in three dimensions and modifying the charge-charge relationship accordingly.

Work has already commenced on the development of new electronics. The University of Wisconsin at Milwaukee has developed electronics that drive and sense over frequencies ranging from 10 to $500 \mathrm{kHz}$. These electronics operate without the use of the Solartron 1260. The electronics measure the phase to within 1 degree of accuracy, which should greatly improve results using biological media.

The electronics are attached to a new data acquisition system that supports three dimensional experiments. The tank has a single column of 15 electrodes surrounded by very large guard rings. The medium under investigation hangs from a fixture. With the aid of a step motor, the fixture moves the medium laterally past the electrodes. A virtual network of electrodes can be simulated depending on the distance each step moves the medium. The system (electronics and acquisition tank) has been operational since the beginning of 2005.

Future experiments will be conducted using Agar media. The use of biological media will be avoided due to the complications associated with contamination of blood with the bath water. 


\section{APPENDIX A: EPET Related Publications}

This appendix contains abstracts for two patents and one short paper. These publications resulted from the work described in this dissertation.

\section{US Patent 6,763,263}

United States Patent

Gregory, et al.

July 13, 2004

Method and apparatus for producing an electrical property image using a charge correlation matrix

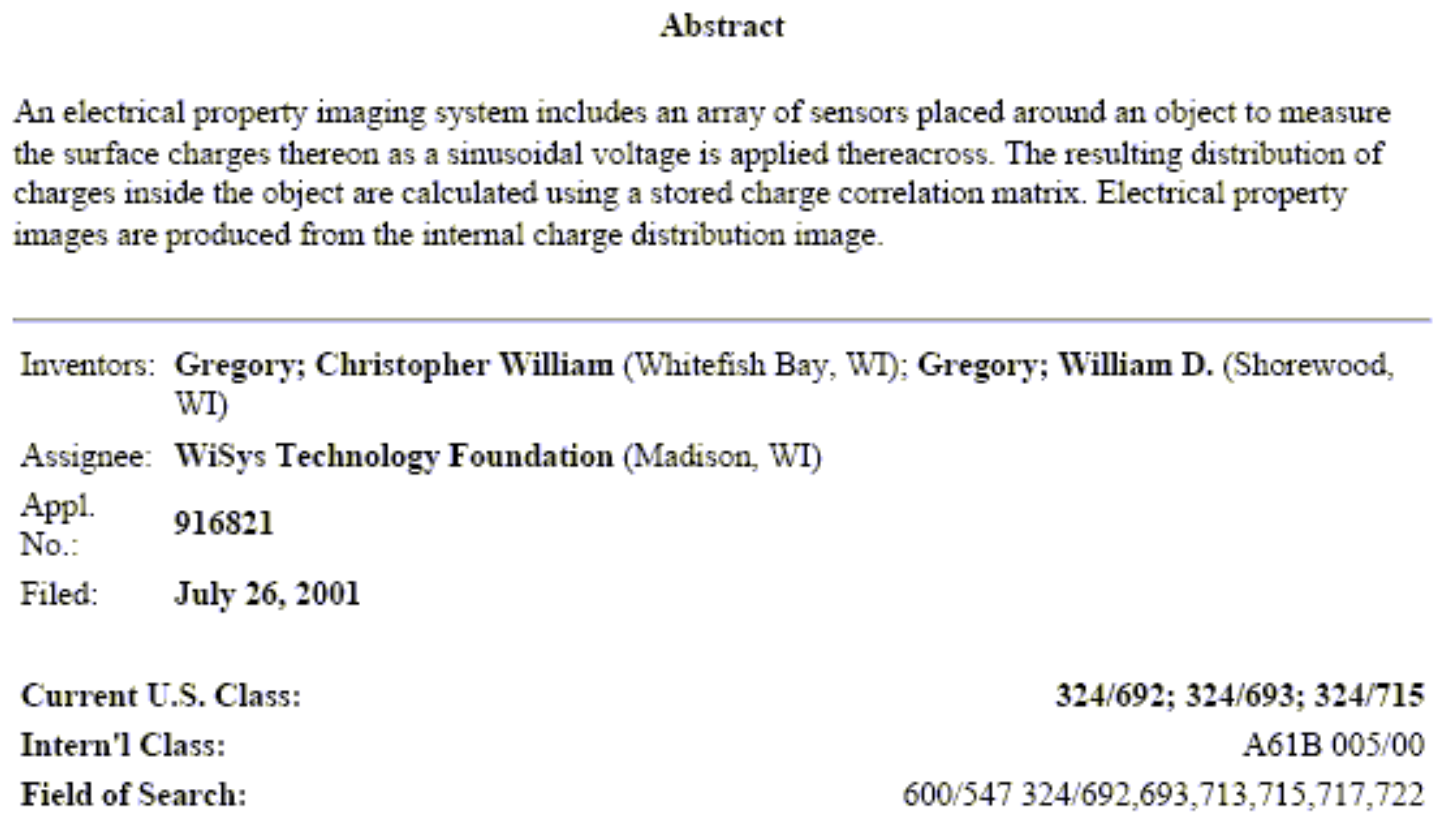


US Patent: 2004/0167421 A1

(19) United States

(12) Patent Application Publication Gregory et al.

(10) Pub. No.: US 2004/0167421 A1

(43) Pub. Date: Aug. 26, 2004

(54) METHOD AND APPARATUS FOR PRODUCING AN ELECTRICAL PROPERTY IMAGE OF SUBSTANTIALLY HOMOGENEOUS OBJECTS CONTAINING INHOMOGENETTIES

(76) Inventors: Christopher William Gregory, Whitefisb Bay, WI (US); William D. Gregory, Shorewood, WI (US)

Correspondence Address:

QUARLES \& BRADY LL.P

411 E. WISCONSIN AVENUE

SUITE 2040

MILWAUKEE, WI 53202-4497 (US)

(21) Appl. No:: 10/700,876

(22) Filed: Nov. 4, 2001

Related U.S. Application Data

(60) Provisional application No, 60/424,568, filed on Nov, 7, 2002 .

Publication Classification

(51) Int. $\mathrm{Cl}^{7}$

(52) U.S. Cl. A61B 5/05

ABSTRACT

An electrical parameter imagine apparatus and method includes the acquisition of a charge distribution pattern on an array of electrodes that surround an object being imaged. In addition the exterior boundary, or contours of the object is measured by an array of light beams and associated light sensors. The contour measurement is employed to provide a first estimate of the object geometry needed to compute an electrical parameter image from the acquired charge distribution pattern. 


\section{Electrical Property Enhanced Tomography (EPET) \\ William D. Gregory ${ }^{1}$ and Christopher W. Gregory ${ }^{2}$}

\begin{abstract}
We report a simulation study of a method for enhancing conventional imaging modalities (CT or MI) by adding information about electrical properties of the object under test. While related to the existing impedance imaging methods ${ }^{3}$, the technique reported here differs in a number of substantive ways. EPET does not attempt to create the image with the electrical data but rather adds electrical property information to the existing modality and, in fact, requires the data from the other modality to locate the position of internal structures in the object". Also, this method arrays the electrode sensors on a fixed geometric strueture outside the object and connects to the object with an electrically conductive bolus. Electrical data are acquired from all sensors simultaneously in an applied voltage rather than an injected current mode. As a result of these and other features of the technique, the image inversion problem is well conditioned and errors propagated from input to output are actually reduced by about two orders of magnitude.
\end{abstract}

Key words - Dielectric constant, conductivity, impedance imaging, error reduction

\section{BACKGROUND}

At the last World Congress in Nice (1997), we outlined a method of extracting images of the interior of an object. reflective of either the object's electrical resistance or reactances. In this paper we extend that work to simultaneous measurement of both components of the object's impedance map, and we report on a detailed simulation study of this imaging modality using four human axial cross sections.

A salient feature of this work is that our method produces a well-conditioned mathematical problem because the solution is expressible in an orthogonal, complete set of functions. In analogy to Fourier spectroscopy, the orthogonality of the solution equations appears to produce a multiplex advantage ${ }^{6}$. In addition, the computing burden is reduced by using other imaging modalities to locate internal structures in the object under test, allowing all the information content of the input data to be used to maximize the accuracy of the impedance image.

As a result of these features, we find a net reduction of noise propagated from input data (sensor currents) to output results (object internal impedance values) of approximately two orders of magnitude.

\section{THEORETICAL STRUCTURE}

Figure 1 illustrates the 2-D geometry we consider for this problem, where $\mathbf{x}_{j}=\left(x_{j}, y_{j}\right)$ [field point] and $\mathbf{x}_{i}^{\prime}=\left(x_{i}^{\prime}, y_{i}^{\prime}\right)$ [source point]. The object under test is thinly coated with an impedance matching gel (the bolus) which connects the object to an array of electrodes situated on a regular geometric structure surrounding the object. (Bolus exaggerated in Fig.1) In the mathematical derivation to follow, the geometry of the electrode array is rectangular. The use of a well defined geometry for the electrode array configuration, rather than the conventional technique of attaching electrodes directly to an arbitrary shaped object, allows the solution of the problem to be expressed in terms of a complete orthogonal set of functions. For the rectangular geometry used here the solution functions are the sine functions, $\sin ((t \cdot \pi \cdot x) / a)$ where $l$ is an integer. Other useful array geometries would be cylindrical (producing Bessel function solutions) and elliptical (leading to elliptical function solutions).

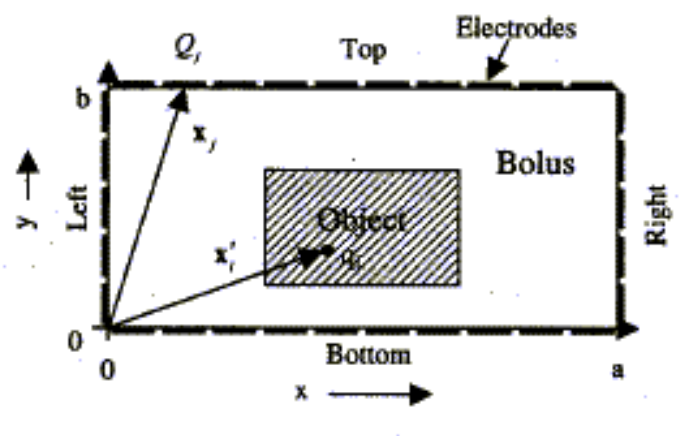

FIGURE 1

We will seek the quasi-static solution to this problem, where $\nabla \times \mathbf{E}=0$, allowing the use of a potential function $\Phi$ to generate $\mathbf{E}$. In addition, we will define the electrical properties of the object under test using the usual constitutive relations to connect the conductivity, $\sigma$, and the relative dielectric constant, $\varepsilon_{r}$, to the $\mathbf{E}$ field. These starting assumptions are summarized as follows:

$$
\mathbf{E}=-\nabla \Phi ; \quad \mathbf{D}=\varepsilon_{r} \varepsilon_{0} \mathbf{E} ; \quad \mathbf{J}=\sigma \mathbf{E}
$$

where $\varepsilon_{0}$ is the dielectric constant of free space.

In addition to the conditions listed in (1), the operable Maxwell equations for this situation are:

$$
\nabla \times \mathbf{H}=\mathbf{J}+\dot{\mathbf{D}} ; \quad \nabla \cdot \mathbf{E}=\frac{\rho_{\text {Total }}}{\varepsilon_{0} .}
$$

Since the divergence of a curl is always zero, the first Maxwell equation in (2) leads to the following equivalent statements in differential and integral form. The integral formulation will be used as the basis for a Finite Difference (FD) Solution to the problem.

$\nabla \cdot\left(\left[\sigma+j \omega \varepsilon_{,} \varepsilon_{r}\right] \nabla \Phi\right)=0$

so using Gauss's law 
$\oint\left(\left[\sigma+j a \varepsilon_{0} \epsilon_{r}\right] \cdot \nabla \Phi\right) \cdot d \mathbf{S}=0$.

Expressing the $\mathbf{E}$ field as the gradient of the potential function per equation (1) and taking the divergence of the second Maxwell equation in (2) leads to the Poisson equation for $\Phi$ :

$$
\nabla^{2} \Phi=-\frac{\rho_{T \text { otal }}}{\varepsilon_{o}}
$$

We will consider a second solution using the Green's function for the Dirichlet boundary conditions to solve (4). The Green's Function (GF) Solution for a closed volume $V$ surrounded by a surface $S$ is given by

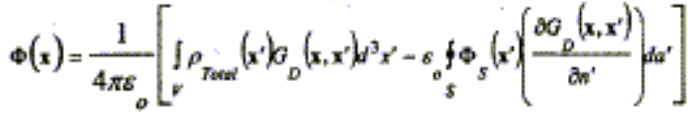

where $n^{\prime}$ is the normal to the surface $S$ and $d a^{\prime}$ is an element of area on $S$.

It is also instructive to write down an expression for the total charge density in the region of interest. This can be obtained by completing the task of taking the divergence of the differential form of equation (3), leading to the following expression:

$$
\rho_{\text {Tam }}=\frac{\left[\nabla \sigma+j \omega \varepsilon_{0} \nabla \varepsilon_{r}\right] \cdot \nabla \Phi}{\left[\sigma+j \omega \varepsilon_{0} \varepsilon_{r}\right]} \cdot \varepsilon_{o}
$$

\section{GREEN'S FUNCTION SOLUTION}

Our methodology will be to complete the Green's Function solution to the problem and use that result as a guide to produce an accurate, easily implemented numerical imaging tool based on the Finite Difference solution.

We start by noting from equation (6) that the total charge density $\rho_{\text {Tad }}$ is non-zero only when (a) either $\nabla \sigma$ or $\nabla \varepsilon_{r}$ is non-zero and (b) not perpendicular to $\nabla \Phi$. This means that if we consider the case where only the bolus is contained in the test region there are no boundaries where the electrical properties change, so $\rho_{\text {now }}=0$ everywhere and the first term in the Green's Function solution (equation (5)) vanishes. Thus, the second term in (5) is just equal to the potential when the test region is empty of the object under test, $\Phi_{t \log }$. For the Dirichlet boundary conditions assumed here, the second term in (5) would remain the same regardless of whether the test object is present or not because the Dirichlet conditions require that the boundary potential, $\Phi_{s}$, must remain constant throughout the experiment. This leads to the following expression for the change of the potential interior to the test volume before and after insertion of the object under test:

$$
\Phi_{\text {Fud }}(\mathbf{x})-\Phi_{\text {Empoy }}(\mathbf{x})=\frac{1}{4 \pi \varepsilon_{0}} \int \rho_{\text {Torof }}\left(\mathbf{x}^{\prime}\right) G_{D}\left(\mathbf{x}, \mathbf{x}^{\prime}\right) d^{3} x^{\prime}
$$

We can now operate on both sides of equation (7) with the del operator and obtain $-\nabla \Phi_{j}=\mathbf{E}_{j}$. Applying Gauss's Law to the $j^{\text {th }}$ sensor surface we obtain:

$$
Q_{j}\left(\mathbf{x}_{j}\right)=\varepsilon_{0}\left[\left.\left(\mathbf{E}\left(\mathbf{x}_{j}\right)\right)\right|_{j}-\left.\left(\mathbf{E}\left(\mathbf{x}_{j}\right)\right)_{j}\right|_{\text {fing }}\right] \cdot \Delta \mathbf{S} \text {, ' where }
$$

$Q_{f}\left(\mathbf{x}_{j}\right)$ is the net charge deposited on $\mathbf{S}$ by the object and oS is an element of area on sensor $j$. We then convert the right side of equation (7) to a discrete sum over the interior charges by setting $\rho_{\text {Tatal }}\left(x_{i}^{\prime}\right) d^{3} x_{i}^{\prime}=q_{i}\left(\mathbf{x}_{i}^{\prime}\right)$ and obtain:

$$
Q_{j}\left(\mathbf{x}_{j}\right)=-\frac{1}{4 \pi} \sum_{j}\left(\nabla G_{D}\left(\mathbf{x}_{j}^{\prime}, \mathbf{x}_{j}\right) \cdot \Delta \mathbf{S}\right]_{s} q_{i}\left(\mathbf{x}_{i}^{\prime}\right)
$$

For the rectangular geometry we have chosen for the sensor array, the Green's Function can be expanded as a sum over two products: (a) sine functions of the field and source variables, $\sin \left(\left(l \cdot \pi \cdot x_{j}\right) / a\right) \cdot \sin \left(\left(l \cdot \pi \cdot x_{i}^{\prime}\right) / a\right)$, where $l$ is an integer, $x_{j}$ is the horizontal component of $\mathbf{x}_{j}$ and $x_{j}^{\prime}$ is the horizontal component of $\mathbf{x}_{i}^{\prime}$ and (b) hyperbolic sine functions of the respective $y$ values in the vertical component direction?. Equation (8) then becomes a set of equations indexed by $j$ and a double sum over $l$ and $i$. Evaluating the normal derivative at the top or bottom boundary for equation (8) eliminates the $y_{j}$ variable on the right side. We can further simplify the remaining sums by taking the spatial sine Fourier transform of the sensor charge values $Q_{j}\left(\mathbf{x}_{j}\right)$. To maximize the information content, we compute the net transform for the top and bottom of the array:

$$
\begin{aligned}
& F T(l)=\int_{0}^{a}\left[Q_{\text {Tap }}(x)-Q_{\text {Bobain }}(x)\right] \cdot \sin \left(\frac{l \cdot \pi \cdot x}{a}\right) d x \\
& F T(l)=\frac{|\Delta \mathrm{S}|}{\pi^{2}} \sum_{T} q_{I} \frac{\sin \left(\left(l \cdot \pi \cdot x_{i}^{\prime}\right) / a\right) \cdot \sinh \left(\left(l \cdot \pi \cdot\left(\frac{b}{2}-y_{l}^{\prime}\right)\right) / a\right)_{(9)}}{\sinh ((l \cdot \pi \cdot b) /(2 \cdot a))}
\end{aligned}
$$

We note that for each Fourier transform, $F T(I)$. we get a separate equation that is a function of a different member of the set of sine functions, $\left\{\sin \left(\left(l \cdot \pi \cdot x_{s}^{\prime}\right) / a\right)\right\}$.

\section{FINITE DIFFERENCE SOLUTION}

Using repeated iterations, we demand that the integral form of equation (3) must be satisfied for the complex potential $\Phi$ at each point within the measuring region. During each iteration the values of the potentials at each point are updated as an average (using equation(3)) of the potentials from the nearest neighbor points. The iterative process is stopped when the changes in potential with each iteration fall below a preset threshold. For boundary potentials of the order of 1 unit, we usually set the threshold to $10^{-8}$. Using a global command in the IDL computer language that allows one to perform a pseudo-parallel operation on all points at once, adding back a portion of the difference between the actual and the required nearest neighbor average at each point to accelerate convergence, and working with a $450 \mathrm{MHz}$ computer, a typical 1000 element array solution will converge in about 10 seconds. This method is an extension of that due to McRae ${ }^{8}$. 


\section{Proceedings of the $22^{\mathrm{Ed}}$ Annual EMBS International Conference, July 23-28, 2000, Chicago IL.}

We have exarnined two methods to utilize the FD solution to determine the values of $\sigma$ and $\varepsilon$. In each case, we solve the "forward" problem i.e., we assume values for $\sigma$ and $\varepsilon$, and then use the computed potentials to compute a set of $F T(l)$ values for a chosen range of $l\left(\right.$ usually $l=1$ to $l_{\max }=$ 25) with the transform now written as a vector $\mathbf{F T}=\left(F T(1), \ldots, F T\left(l_{\max }\right)\right)$. We define an electrical properties vector alpha $\alpha$ as follows:

$$
\alpha=\left(\varepsilon_{r}(k=1), \ldots, \varepsilon_{r}\left(k=k_{\max }\right), \sigma(k=1), \ldots, \sigma\left(k=k_{\max }\right)\right)
$$

where the index $k$ counts the number of different impedance values for the object under test (i.e., skin, muscle, bone, etc.). We assume all regions of the same tissue type in a given cross-section have the same electrical values. The two methods are summarized in equations (10) and (11):

$$
\begin{gathered}
\text { Data Base Method } \\
\mathbf{F T}_{\text {Mear }}=\mathbf{F T}_{\text {Scard }} \cdot \mathbf{S C} \\
\left\langle\mathbf{F T}_{\text {Mear }}\right\} \leftrightarrow\left\{\mathbf{F T}_{\text {Sunad }}\right\} \\
\text { Iterative Correction Method } \\
\mathbf{F T}_{\text {Meax }}-\mathbf{F T}_{\text {Tial }}=\frac{\partial \overline{\mathbf{F T}}}{\partial \alpha} \cdot \delta \alpha
\end{gathered}
$$

In the Data Base Method we solve the problem for a given situation (e.g. for a typical chosen human axial cross section) using average literature values for the electrical properties, and from this solution we compute a "standard" FT vector. FT $_{\text {Sand }}$. We then compare the measured FT vector FT $_{\text {Dess }}$ to $\mathbf{F T}_{\text {Sasd }}$ using a scale factor vector $\mathbf{S C}$, where the elements of $\mathbf{S C}$ are the factors one must increase (decrease) $\mathbf{F T}_{\text {Stand }}$ to make it equal to $\mathbf{F T}_{\text {Mes }}$ These computations can be performed off-line for a large number of cases and then the set of vectors $[\alpha]$ can be correlated to the set of possible measured transforms \{ FT $_{\text {Mess }}$ \} using either a look-up table or a neural net.

In the Iterative Correction Method we use the actual CT or MI cross-section of the object to compute a trial transform, $\mathbf{F T}_{\text {Tris: }}$. The difference between $\mathbf{F T}_{\text {Mess }}$ and $\mathbf{F} \mathbf{T}_{\text {Triel }}$ is used to compute a corroction to the electrical property vector, $\delta \alpha$, using the first term in a Taylor's series expansion of the change of $\mathbf{F T}_{\text {Thal }}$ given by a numerically calculated matrix of dimensions $k_{\text {Max }}$ by $l_{\text {Mx }}$ written as $\frac{\partial \overline{\mathbf{F T}}}{\partial \alpha}$. We obtain $\delta \alpha$ by inverting equation (11) with a typically over-determined set of equations utilizing a Singular Value Decomposition technique?. If the magnitude of $\delta \alpha$ is larger than a preset value, $\delta \alpha$ is used to re-compute $\mathbf{F} \mathbf{T}_{\text {Thial }}$ and the process is repeated until the change in $\alpha$ is below an acceptable limit. A flow chart of the Iterative Correction Method is shown in Figure 2.

\section{RESULTS AND DISCUSSION}

We have conducted simulation studies of both the Data Base and Iterative Correction methods using anatomical data on four human cross-sections from the Visible Human Project ${ }^{10}$ : legs, pelvis, thorax and head. Values for the dielectric constant and conductivity of human tissues were

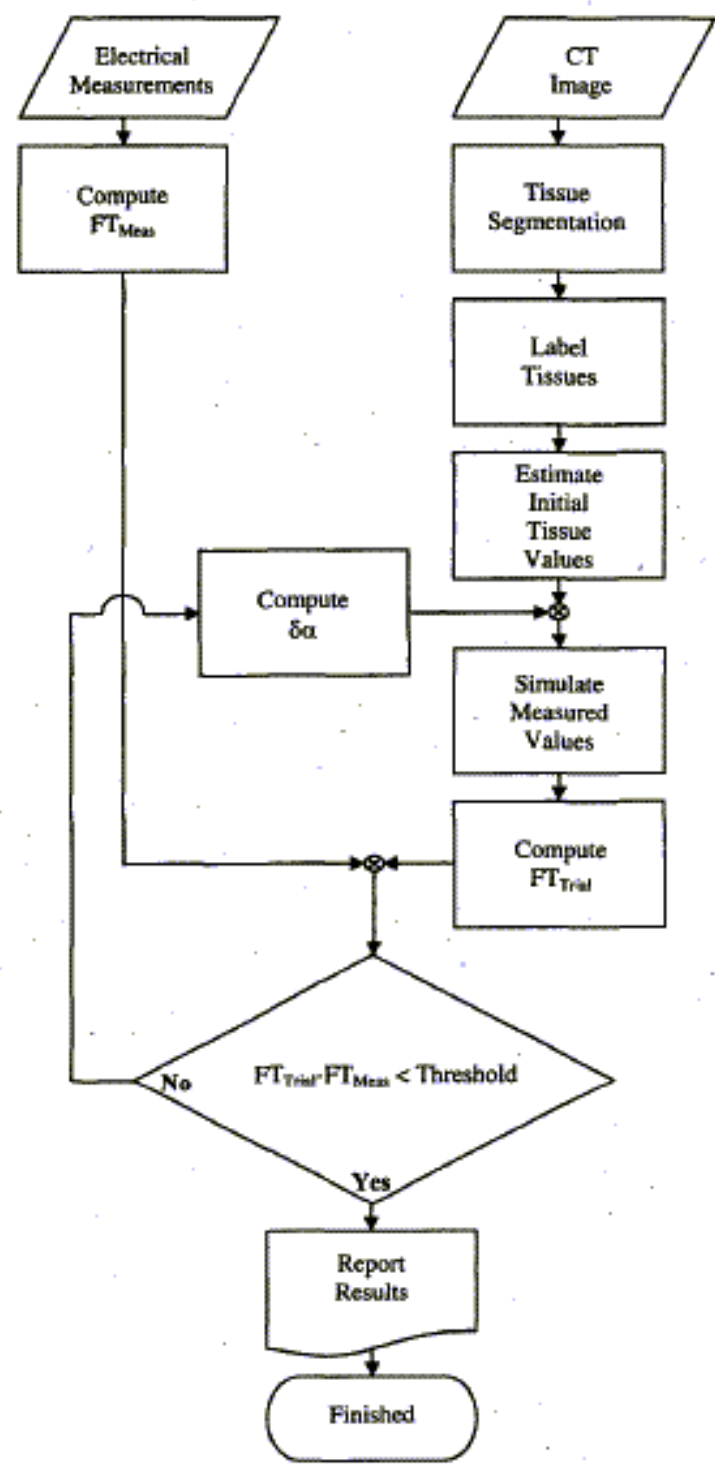

estimated using the compilation of Gabriel, Lau and Gabriel ${ }^{\mathrm{HI}}$ for five frequencies: $10^{4} .10^{5}, 10^{6}, 10^{7}$ and $10^{8} \mathrm{~Hz}$. Our simulation software allowed us to include noise or errors introduced by changes of the object (such as might be caused by motion of a human subject, perfusion of blood in organs, respiratory cycle changes, etc.) and/or due to inaccuracy of the current measurement. In addition, we were able to study the effect of various combinations of data from different relative orientations of the object and the electric field, as well as the effect of bolus impedance on the propagation of error. The basic methodology of the simulations was to 
Proceedings of the $22^{\text {nd }}$ Annual EMBS International Conference, July 23-28, 2000, Chicago IL.

define a "true" value for the electrical properties of the crosssection and then determine bow close either technique could come to the "true" values. In particular, we studied the effects of choosing "trial" values (as defined above) that were not necessarily close to the "true" value.

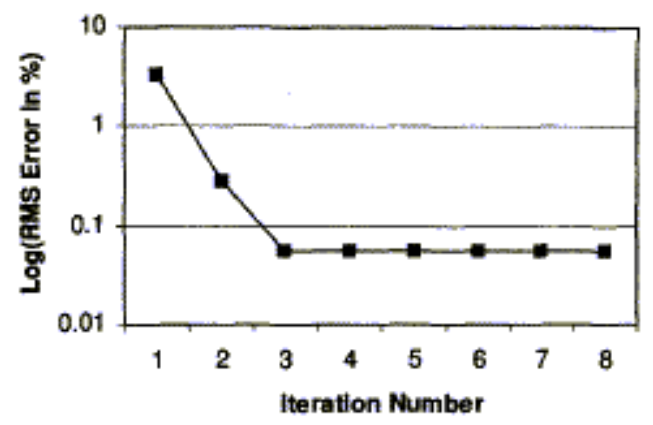

FIGURE 3

To investigate the Data Base method we generated a file of $6000 \mathrm{FT}$ vectors for a range of electrical property values bracketing a nominal "standard" value. Using both a look-up table approach and a neural net analysis we found that we could typically come within a few percent rms difference of the "true" and "measured" tissue electrical property values with this approach. While it is computationally intensive to produce the database, those calculations can be done off-line. Particularly with the neural net approach, a final result for a test query can be obtained almost instantaneously once the neural network algorithm is generated.

The Iterative Correction method yielded even more accurate results. Figure 3 shows the convergence of the rms difference between the "true" electrical property values and the "trial" values for successive iterations of equation (11). A pelvic cross-section with random errors of about $1 \%$ added to the object electrical property values for the "measured" transforms were used for the case shown in Figure 3.

We note in Figure 3 that after only three iterations the result converges to within $0.05 \% \mathrm{rms}$ of the "true ${ }^{*}$ electrical property values. This residual is due only to the random error introduced on the input. When we introduce zero random errors on the input, the values converge to within the double precision accuracy of the computer. Moreover, we found that the convergence was unaffected by the magnitude of the random error introduced or the deviation of the original "trial" values guessed, up to about at least a factor of two orders of magnitude times the "true" values.

We see the database method as a good way to generate starting $\sigma$ and $\varepsilon_{r}$ values from typical cross-sections to use with the iterative methad for the actual cross-section.

At the beginning of this study we had assumed that the proper values for the electrical properties of the bolus would be approximately equal to those of the outer surface of the object (i.e., the skin). In fact, choosing a bolus with the skin values results in almost a 1:1 ratio of "error out vs. error in ". We used the Pówel] ${ }^{9}$ multidimensional minimization procedure to obtain the bolus impedance values that minimize the error propagation for all of the cross-sections used in this study at all frequencies employed. Table I lists the best and worst cases of "error out vs. error in" for the entire study, The "error out/error in" ratios $\left(e_{\text {pue }} / e_{i s}\right)$ in Table I range from $0.7 \times 10^{-2}$ to $6 \times 10^{-2}$. For the number of measurements used in each simulation, this is about the error reduction expected from the multiplex advantage.

TABLE I

"ERROR OUT/ERROR IN" WITH OPTIMUM BOLUS

\begin{tabular}{|c|c|c|c|c|}
\hline 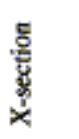 & $\begin{array}{l}\text { 졸 } \\
\text { 总 } \\
\text { 至 }\end{array}$ & $\stackrel{5}{\frac{5}{3}}$ & $\begin{array}{l}\text { 졸 } \\
\text { 总 } \\
\text { 点 }\end{array}$ & 胥 \\
\hline Head & $10^{4}$ & $1.66 \times 10^{-2}$ & $10^{6}$ & $2.83 \times 10^{-2}$ \\
\hline Chest & $10^{5}$ & $2.31 \times 10^{-2}$ & $10^{8}$ & $2.68 \times 10^{-2}$ \\
\hline Pelvis & $10^{6}$ & $0.74 \times 10^{-2}$ & $10^{4}$ & $6.11 \times 10^{-2}$ \\
\hline Legs & $10^{4}$ & $3.09 \times 10^{-2}$ & $10^{8}$ & $3.12 \times 10^{-2}$ \\
\hline
\end{tabular}

This work supported in part by NASA, the WV-NSF EPSCoR program and the WVU Virtual Reality Lab.

\section{REFERENCES}

\section{Dess of Enginetring}

WVU Toch, Leanard C. Nelson College of Engineering

Moetgomary, WV 25136

Wgregory wivutech.edu

2. Department of Computer Science and Electrical Enginecring WVU. College of Engineering and Mineral Resources Morgantown. WV 26506

Cwgregory a aol.com

3. Merueci, J-P., Valentuzzi. B. R., Fetice, C., L. Chavesu, N., and Marsili. P.M., 1996 "Biolectrio impedance techniques in medicine", Critical Reviews in Biomedical Engineering 24, no. 4-6, 223-681

4. Glidewell and $\mathrm{Ng}$ have also used anaturnical information to improve impedance tomograpty: Glidewell, M., and $\mathrm{Ng} . \mathrm{K} . \mathrm{T}, \mathrm{s}$, 199 -Anatcenically constrained electrical impedance tormography for anisotropic bodies va a two-step spproach", IEEE Transacrians an Medtcal lmaging 14, no. 3.498.503

5. Gregory. W. D. McRas, D. A. and Gregary, C. W., 1997 'Ekectrical property tamograpty using the correlatico of exterion-to-interiot induced currents", J. of the lint. Fed for Med \& Bio. Engineering 35 , (Supplement, part D, 325

6. Vanasse, G. A., Stair, A. J., Jf., and Baker, D. J., eds., Aspen intementianal Canference an Fourier Spectrascopy. 1970, Special Repors No. 114, AF Cambridge Res, Lab, AFCRL 71-0019, 1971, 3

7. Jackson, J. D., Classicel Electrodymamics, 2N Ed. John Wiley \& Sons, 1975,121

8. McRae, D. A. "A technique for modeling the internal struebares of objocts using phase sensitive measurement of their low-frequency electrical respouse and its spplicalion to some human body cross. sections", Ph.D. thesis, Georgetom University, 1984 (Uhily. Mistrofilms\})

9. Press, W. H. Flanoery, B. P. Teukolsky, S. A., and Vetterling, W. T., Numerical Recipes, Cambridge University Press, 1986

10. Images svailable on CD-ROM from RSL, lhc, 2995 Wildemess Place. Boalder CO \$a 01 (USA)

11. Gabriel, S., Lau, R. W., and Gabriel, C., 1996 "The dielectric properties of biological tissues: III, Parametric models for the dielectrie spectrum of tissues", Phys. Med. Biol. 41, 2271-2293 


\section{APPENDIX B: Using FDM to Model the Uniform Case Potential}

The uniform potential, $\Phi_{\mathrm{u}}$, is used in the computation of the charge-charge correlation method (equation (3-43)) and in the computation of the relative current (equation (5-1)). Since the uniform case is a single-medium case (i.e. only saline is measured), its computation is trivial. The finite difference method is employed to develop a continuous model of the potential field, $\Phi(\mathrm{x}, \mathrm{y})$. The gradient of this model is then determined by analytical means. The result is employed in equations (3-43) and (5-1).

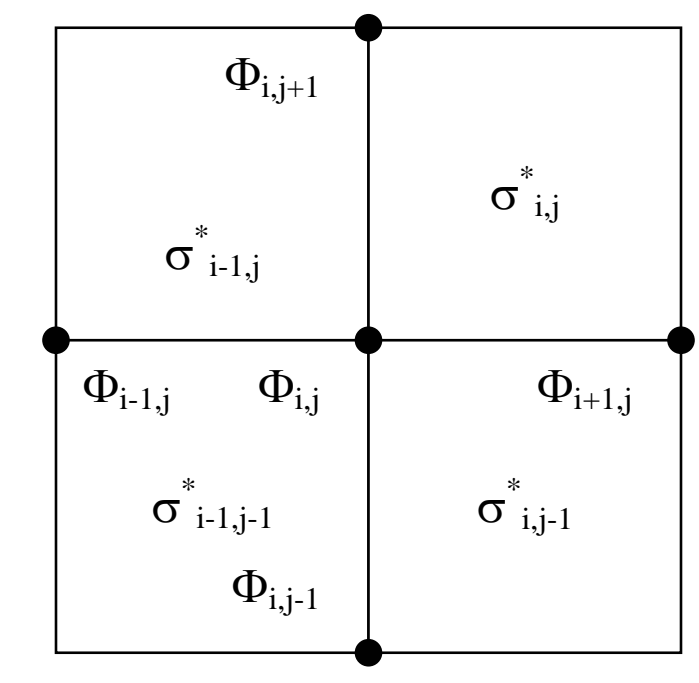

Figure B-1: Discritized potential locations within a domain.

The finite difference is a numerical method that computes the potential at discrete locations in the domain (equations (B-1) and (B-2)). Figure B-1 illustrates the layout of five discrete potential points. The potential points are labeled $\Phi_{\mathrm{i}, \mathrm{j}}$, where the indices $\mathrm{i}$ and $\mathrm{j}$ correspond to the discrete $\mathrm{x}$ and $\mathrm{y}$ locations respectively (defined in equations (B-3) through (B5)). Because the electrical properties, $\sigma_{i, j}$, in equation (B-2) are all the same value, the finite difference potential computation simplifies to one involving only the potential values (equation (B-6)). 


$$
\begin{aligned}
& \Phi_{i, j}=\Phi\left(x_{i}, y_{j}\right) \\
& \Phi_{i, j}^{n+1}= \\
& \frac{\left(\sigma_{i, j-1}^{n}+\sigma_{i, j}^{n}\right) \Phi_{i+1, j}^{n}+\left(\sigma_{i-1, j}^{n}+\sigma_{i, j}^{n}\right) \Phi_{i, j+1}^{n}+\left(\sigma_{i-1, j}^{n}+\sigma_{i-1, j-1}^{n}\right) \Phi_{i-1, j}^{n}+\left(\sigma_{i, j-1}^{n}+\sigma_{i-1, j-1}^{n}\right) \Phi_{i, j-1}^{n}}{4 \cdot\left(2\left(\sigma_{i, j-1}^{n}+\sigma_{i, j}^{n}+\sigma_{i-1, j-1}^{n}+\sigma_{i-1, j}^{n}\right)\right)} \\
& \text { where } \mathrm{i} \text { is the column } \\
& \mathrm{j} \text { is the row } \\
& \mathrm{n} \text { is the iteration count }
\end{aligned}
$$

$$
\begin{gathered}
x_{i}=i \cdot \delta x \\
y_{j}=j \cdot \delta y \\
\delta x=\delta y=\frac{23.1 \mathrm{~cm}}{93} \\
\Phi_{i, j}^{n+1}=\frac{\Phi_{i+1, j}^{n}+\Phi_{i, j+1}^{n}+\Phi_{i-1, j}^{n}+\Phi_{i, j-1}^{n}}{4}
\end{gathered}
$$

The potential is computed on the square domain $(23.1 \mathrm{~cm}$ on a side) that is discretized into 93 by 93 potential points. The resulting discrete potential values are used compute a twodimensional spatial polynomial fit of the domain potential. The fit is computed using the IDL function sfit.pro, which determines a polynomial fit to a surface and returns a fitted array. This polynomial fit, defined in equation (B-7), uses the coefficient kx, defined in equation (B-8). The gradient of the potential is determined analytically in equation (B-9), which is used in equation (3-43).

The boundary current along the $y=0$ side of the sample holder is computed from the integral of the current density passing through a given electrode centered at $\mathrm{x}_{\mathrm{k}}$ using the gradient of the potential (equation (B-9)). This integral, equation (B-10), is simplified in equation (B-11).

$$
\begin{gathered}
\Phi(x, y)=\sum_{i=0}^{5} \sum_{j=0}^{5} k x(i, j) \cdot x^{i} \cdot y^{j} \\
k x=\left(\begin{array}{cccccc}
9.88 \mathrm{E}-01 & -1.43 \mathrm{E}-02 & -1.14 \mathrm{E}-04 & 5.63 \mathrm{E}-06 & -7.05 \mathrm{E}-08 & 2.99 \mathrm{E}-10 \\
1.17 \mathrm{E}-03 & -1.36 \mathrm{E}-05 & 1.33 \mathrm{E}-05 & -4.94 \mathrm{E}-07 & 6.39 \mathrm{E}-09 & -2.83 \mathrm{E}-11 \\
-5.11 \mathrm{E}-05 & 5.21 \mathrm{E}-06 & -5.89 \mathrm{E}-07 & 1.93 \mathrm{E}-08 & -2.47 \mathrm{E}-10 & 1.09 \mathrm{E}-12 \\
7.93 \mathrm{E}-07 & -1.07 \mathrm{E}-07 & 9.57 \mathrm{E}-09 & -3.03 \mathrm{E}-10 & 3.86 \mathrm{E}-12 & -1.71 \mathrm{E}-14 \\
-3.55 \mathrm{E}-09 & 5.16 \mathrm{E}-10 & -5.05 \mathrm{E}-11 & 1.64 \mathrm{E}-12 & -2.11 \mathrm{E}-14 & 9.39 \mathrm{E}-17 \\
-6.05 \mathrm{E}-12 & 4.86 \mathrm{E}-13 & -9.87 \mathrm{E}-15 & 4.72 \mathrm{E}-18 & 1.39 \mathrm{E}-18 & -8.85 \mathrm{E}-21
\end{array}\right) \\
\nabla \Phi(x, y)=\sum_{i=0}^{5} \sum_{j=0}^{5} i \cdot k x(i, j) \cdot x^{i-1} \cdot y^{j} \hat{\mathbf{i}}+\sum_{i=0}^{5} \sum_{j=0}^{5} j \cdot k x(i, j) \cdot x^{i} \cdot y^{j-1} \hat{\mathbf{j}}
\end{gathered}
$$




$$
\begin{gathered}
I_{u}\left(x_{k}, 0\right)=-\left.\sigma_{1}^{*} \cdot \int_{x_{k}-\Delta x}^{x_{k}+\Delta x} \int_{0}^{\sqrt{R}} \nabla \Phi\right|_{\left(x_{k}, 0\right)} d z^{\prime} d x^{\prime} \cdot \hat{\mathbf{y}} \\
I_{u}\left(x_{k}, 0\right)=-\sigma_{1}^{*} \cdot \sum_{i=1}^{6} k x(i, 0) \cdot \int_{x_{k}-\Delta x}^{x_{k}+\Delta x} x^{\prime i} \cdot \sqrt{R-x^{\prime 2}} d x^{\prime}
\end{gathered}
$$




\section{APPENDIX C: Observed Current Pattern Dependence on Lateral Position}

Recall that the peak current is selected from 1 of 15 electrodes. The electrode corresponding to the peak current depends on the lateral location of medium 2. The predicted and measured peak currents oscillate in magnitude as a function of the x-position of medium 2. To explain this behavior, imagine if the 15 electrodes are replaced with a large number of electrodes. The measured current pattern would appear to be a continuous function. Such a continuous function is illustrated in Figure C- 1 with a solid line. In this example, $\mathrm{x}_{2}=11.6 \mathrm{~cm}$ (directly in front of electrode 8), $\mathrm{y}_{2}=2.4 \mathrm{~cm}, \mathrm{~d}_{2}=1.9 \mathrm{~cm}$, and $\kappa_{21}=10^{7}$. As the number of electrode is reduced, it effectively discretizes the continuous current pattern. This discretization

effectively occurs only at the center of the electrode (dashed line). Figure C-2 illustrates the case when the sample is moved between electrode 7 and $8\left(x_{2}=10.8 \mathrm{~cm}\right)$. The peak of the of the continuous current pattern (solid line) shifts to the left, but the discretize current pattern (dashed red line) peak disappears. The peak appears to be truncated and is shared by electrodes 7 and 8 . The value of the peak is reduced as well when compared to Figure C-1, consequently, the peak current is expected to oscillate as the sample moves along the $\mathrm{x}$-direction. 


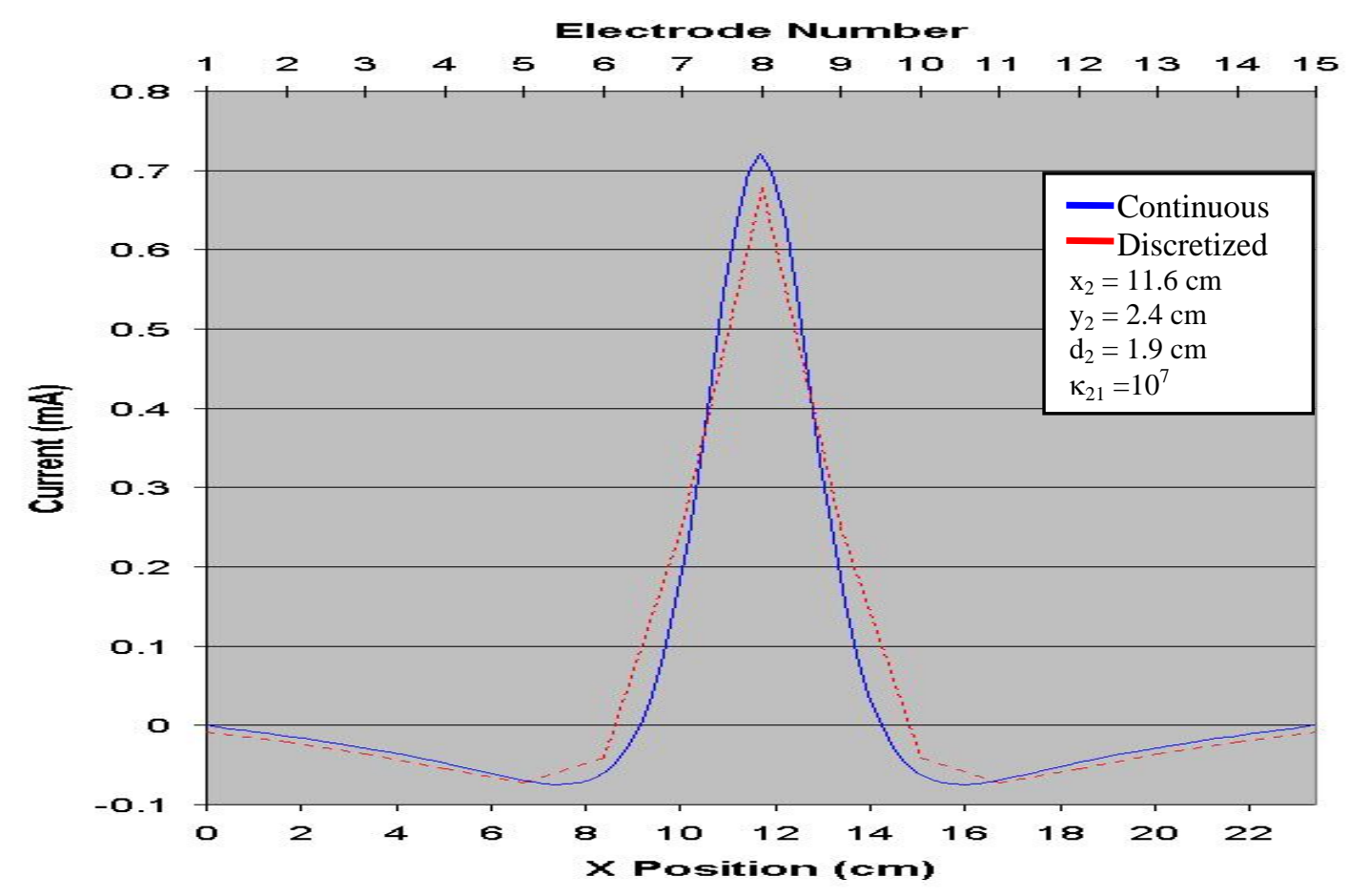

Figure C-1: Comparing continuous and discretized current patterns - Sample position centered on the electrode.

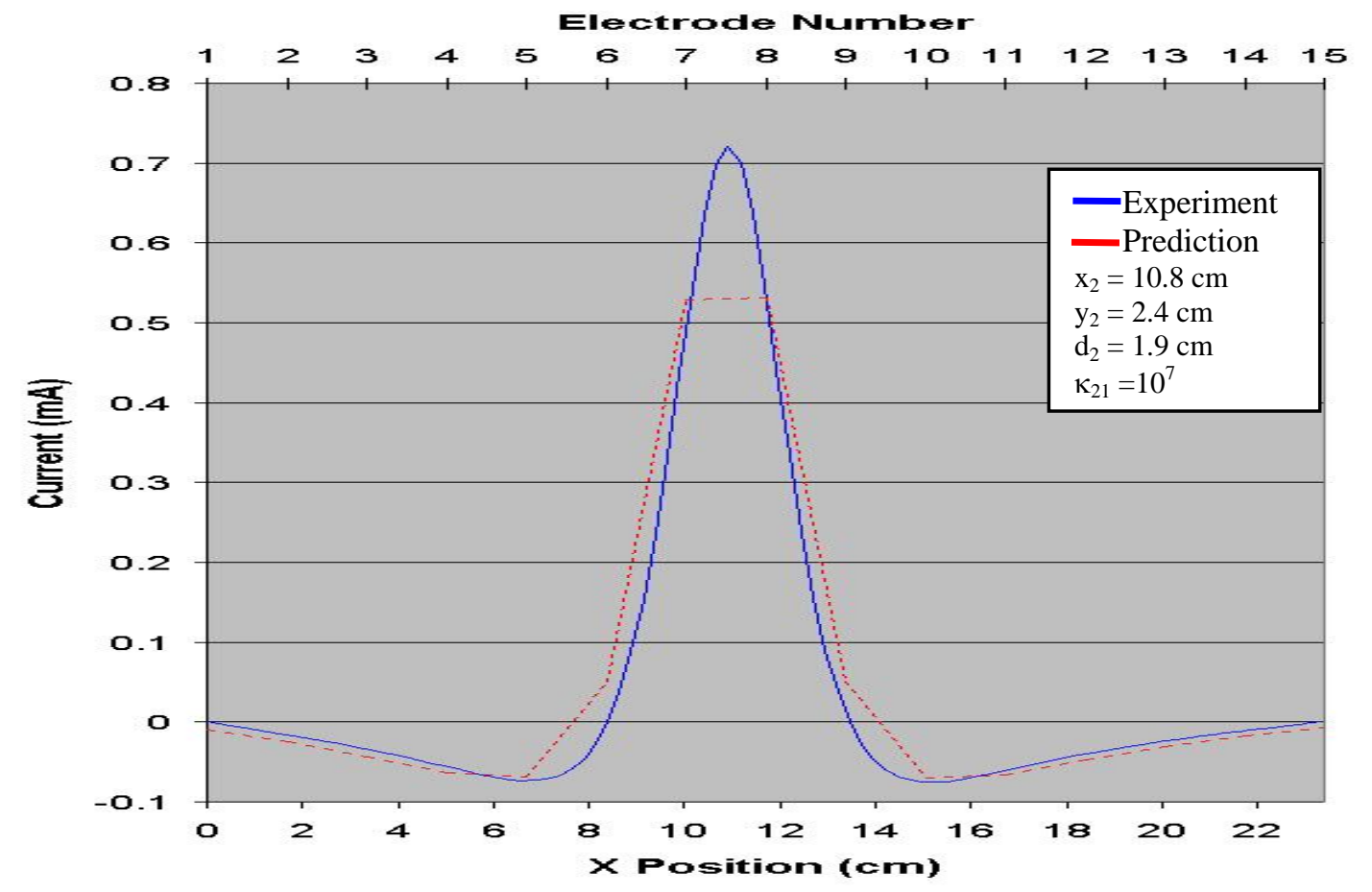

Figure C-2: Comparing continuous and discretized current patterns - Sample position between electrodes 


\section{CURRICULUM VITAE}

\section{EDUCATION}

Ph.D., Electrical Engineering

West Virginia University

Lane Department of Computer Science, Electrical and Computer Engineering

Morgantown, $W V$

Dissertation Title: The use of charge-charge correlation in impedance measurements: a test of the EPET method.

Advisor: Dr. Bojan Cukic, Ph.D.

M.S., Control Systems Engineering

West Virginia University Institute of Technology

Control Systems Engineering Program

Montgomery, WV

M.S. Project Title: Queue System Control: An Adaptive Control Scheme

M.S., Physics

1990

University of Illinois

Department of Physics

Urbana, IL

B.S., Physics

Clarkson University

Department of Physics

Potsdam, NY

PROFESSIONAL EXPERIENCE

University of Wisconsin-Milwaukee, Milwaukee, WI

2000 to Present

and

West Virginia University, Morgantown, WV (Joint Appointment)

Associate Researcher:

Conducted research in Electrical Property Enhanced Tomography (EPET), a variation Electrical Impedance Imaging. 
- Conceived, designed, generated, tested and verified software for processing and analyzing Electrical Property Enhanced Tomography Data for breast cancer models.

- Conceived, designed, and implemented methodologies for measuring and analyzing EPET data of breast cancer models.

- Co-authored multiple proposals to research in breast cancer screening and explosives detection.

- Supervised graduate and undergraduate students, as well as support personnel in EPET research.

Institute for Software Research, Fairmont, WV

1999 to 2000

\section{Member of the Research Staff.}

Collaborated in a group effort to develop a proposal to NASA's Dryden facility for work on fault tolerant flight control systems.

- Conducted analysis of the ARSHUS program which resulted in a Concept of Operations document. ARSHUS (Aerial Remote Sensing of Hydrologic Systems Under Stress) will use a suite of sensors placed on an unmanned aerial vehicle to collect data about the hydrologic, environmental and agricultural condition of the Mid-Atlantic region.

ANSER, Fairmont, WV

1998-1999

\section{Research Engineer for Image Recognition.}

Conducted analysis on the influence of light variations in facial recognition.

- Developed an algorithm for extracting photos from bitmap images.

- Designed and implemented a multi-camera experimental set-up for video recognition.

West Virginia University, Morgantown, WV

$1997-1998$

Teaching Assistant - Computer Science and Electrical Engineering Department·

Instructed a two semester, hands-on course for microprocessor interfacing. The course covered machine language, assembly language, hardware and software interfacing, memory $\mathrm{I} / \mathrm{O}$, and basic microprocessor interfacing techniques.

West Virginia University Institute of Technology, Montgomery, WV

$1995-1997$

Research Assistant - Leonard C. School of Engineering.

Conducted research on dielectric imaging and developed computer programs for IDL which simulate the data fusion of CT scans and dielectric imaging techniques.

The Johns Hopkins University, Applied Physics Laboratory, Laurel, MD 1991 - 1995 


\section{Associate Professional Staff Engineer-}

- Developed and tested new and established software code for processing and analyzing in situ acoustic data collected on US naval sea tests.

- Generated a computer simulator of the AEGIS Class radar target tracking function in support of US naval anti-aircraft warfare research.

- Conducted matched-filtering and other signal processing analysis of acoustic data in support of US naval anti- submarine warfare research.

- Published internal documents and presented results to sponsors and customers.

University of Illinois, Urbana, IL

Research Assistant - Atmospheric Sciences Department•

Processed atmospheric data and created image-based animation in support of atmospheric research.

Teaching Assistant - Physics Department•

Instructed undergraduate physics courses for five semesters. 


\section{REFERENCES}

1 A. J. Surowiec, S. S. Stuchly, J. R. Barr and A. Swarup, "Dielectric properties of breast carcinoma and the surrounding tissues,” IEEE Transactions on Biomedical Engineering, vol. 35(4), pp. 257-263, April 1988.

2 T. J. Yorkey, "Comparing reconstruction methods for electrical impedance tomography," Ph.D. dissertation, University of Wisconsin, Madison, WI, 1986.

3 W. D. Gregory, D. A. McRae and C. W. Gregory, "Electrical Property Tomography using the correlation of exterior-to-interior induced currents,” presented at the World Congress on Medical Physics and Biomedical Engineering, Nice, France, 1997.

4 W. D. Gregory and C. W. Gregory, "Electrical Property Enhanced Tomography (EPET)," in the Proceedings of the 22nd Annual International Conference of the IEEE Engineering in Medicine and Biology Society, vol. 4, pp. 2632 - 2635, 2000.

5 G. N. Stewart, "Research on the circulation time in organs and on the influences which affect it,” Journal of Physiology, vol. 15, pp. 1-89, 1894.

6 G. N. Stewart, "The measurement of the output of the heart," Science, vol. 22, p. 137, 1897

7 R. Höber, “Ein zweites Verfahren, die Leitfähigkeit im Innern von Zellen zu messen,” Arch. f. d. ges. Physiol., vol. 148, pp. 189, 1912.

8 G. W. Crile, H. Hosmer and A. F. Rowland, "The electrical conductivity of animal tissues under normal and pathological conditions,” American Journal of Physiology, vol. 60(1), pp. 59-106, 1921.

9 H. Fricke and S. Morse, “The electrical capacity of tumours of the breast,” Journal of Cancer Reserarch, vol.10, pp. 340-376, 1926.

10 K. S. Cole and R. H. Cole, "Dispersion and absorption in dielectrics," Journal of Chemical Physics, vol. 9, pp. 341-351, 1941.

11 K. S. Cole and H. J. Curtis, "Electrical physiology: Electrical resistance and impedance of cells and tissues,” in Medical Physics, Chicago: Yearbook Publishers, 1944, pp. 344348.

12 H. P. Schwan, "Electrical properties of tissues and cell suspensions," in Advances in Biological and Medical Physics. Vol. 5, p. 147-209. Academic Press. New York.

13 K. R. Foster and H. P. Schwan, "Dielectric properties of tissues and biological medias. A critical review,” Critical Reviews in Biomedical Engineering, vol. 17, pp. 25-104, 1989.

14 L. A. Geddes and L. E. Baker, "The specific resistance of biological medias-a compendium of data for the biomedical engineer and physiologist,” Medical and Biological Engineering, vol. 5, pp. 271-293, 1967.

15 C. Gabriel, S. Gabriel, and E. Corthout, "The dielectric properties of biological tissues .1. Literature survey,” Physics in Medicine and Biology, vol. 41(11), pp. 2231-2249, November 1996.

16 S. Gabriel, R. W. Lau and C. Gabriel, "The dielectric properties of biological tissues: III. Parametric models for the dielectric spectrum of tissues,” Physics in Medicine and Biology, vol. 41(11), pp. 2271-2293, November 1996.

17 Italian National Research Group, Institute for Applied Physics, “ Dielectric Properties of Body Tissues," http://niremf.ifac.cnr.it/tissprop. 
18 B. Rigaud, J. -P. Morucci and N. Chauveau, "Bioelectrical impedance techniques in Medicine, Part I, Second Section,” Critical Reviews in Biomedical Engineering, vol. 24(46), pp. 257-351, 1996.

19 R. Pethig, "Dielectric properties of body tissues," Clinical Physics and Physiological Measurement, vol. 8, pp. A3-A12, 1987.

20 V. Raicu, "Dielectric dispersion of biological matter: Model combining De Bye - type and 'universal' responses,” Physical Review E, vol. 60(4), pp. 4677 - 4680, October 1999.

21 J.G. Webster, Ed., Electrical Impedance Tomography, New York: Hilger, 1990.

22 R. C. Weast, M. J. Astle and W. H. Beyer, Ed. CRC Handbook of Chemistry and Physics, 65th Edition, Boca Raton: CRC Press, 1984.

23 K. S. Osterman, T. E. Kerner, D. B. Williams, A. Hartov, S. P. Poplack and K. D. Paulsen, "Multifrequency electrical impedance imaging: preliminary in vivo experience in breast,” Physiological Measurement, vol. 21(1) pp. 99-109, 2000.

24 B. Blad and B. Baldetorp, "Impedance spectra of tumor tissue in comparison with normal tissue; a possible clinical application for electrical impedance tomography,” Physiological Measurement, vol. 17, pp. A105-A115, 1996.

25 J. Jossinet, “The impedivity of freshly excised human breast tissue,” Physiological Measurement, vol. 19, pp. 61-75, 1998.

26 J. Jossinet, "Variability of impedivity in normal and pathological breast tissue," Medical \& Biological Engineering \& Computing, vol. 34(5), pp. 346-350, September 1996.

27 J. Jossinet and M. Schmitt, "A review of parameters for the bioelectrical characterization of breast tissue,” Annals of the New York Academy of Sciences, vol. 873, pp. 30-41 1999.

28 J. E. da Silva, J. P. M. de Sa and J. Jossinet, "Classification of breast tissue by electrical impedance spectroscopy,” Medical \& Biological Engineering \& Computing, vol. 38(1), pp. 26-30, January 2000.

29 R. Pethig, Dielectric and Electric Properties of Biological Medias, Chichester: John Wiley, 1979.

30 E. Zheng, S. Shao and J. G. Webster, "Impedance of skeletal muscle from $1 \mathrm{~Hz}$ to 1 MHz,” IEEE Transactions on Biomedical Engineering, vol. 31, pp. 477-481, 1984.

31 K. D. Paulsen and J. Jiang, "An enhanced electrical impedance imaging algorithm for hyperthermia applications,” International Journal of Hyperthermia, vol. 13(5), pp. 459480 September-October 1997.

32 B. R. Epstein and K. R. Foster, “Anisotropy in dielectric properties of skeletal muscle," Medical \& Biological Engineering \& Computing, vol. 21, pp. 51-55, 1983.

33 C. A. Shiffman and R. Aaron, "Angular dependence of resistance in non-invasive electrical measurements of human muscle: the tensor model,” Physics in Medicine and Biology, vol. 43(10), pp. 1317-1323, May 1998.

34 R. Aaron, M. Huang and C. A. Shiffman, "Anisotropy of human muscle via noninvasive impedance measurements,” Physics in Medicine and Biology, vol 42(7), pp. 1245-1262, July 1997.

35 M. Assenheimer, O. Laver-Moskovitz, D. Malonek, D. Manor, U. Nahaliel, R. Nitzan and A. Saad, "The T-SCAN (TM) technology: electrical impedance as a diagnostic tool for breast cancer detection,” Physiological Measurement, vol. 22(1), pp. 1-8, February 2001. 
36 A. Malich, T. Fritsch, R. Anderson, T. Boehm, M. G. Freesmeyer, M. Fleck and W. A. Kaiser, "Electrical impedance scanning for classifying suspicious breast lesions: first results,” European Radiology, vol. 10(10), pp. 1555-1561, 2000.

37 A. Malich, T. Boehm, M. Facius, M. Freesmeyer, M. Fleck, R. Anderson and W. A. Kaiser, "Additional value of electrical impedance scanning: experience of 240 histologically-proven breast lesions,” European Journal of Cancer, vol. 37(18), pp. 23242330, December 2001.

38 A. Malich, D. Sauner, C. Marx, M. Facius, T. Boehm, S. O. Pfleiderer, M. Fleck and W. A. Kaiser, "Influence of breast lesion size and histologic findings on tumor detection rate of a computer-aided detection system,” Radiology, vol. 228(3), pp. 851-856, September 2003.

39 V. A. Cherepenin, A. Y. Karpov, A. V. Korjenevsky, V. N. Kornienko, Y. S. Kultiasov, M. B. Ochapkin, O.V. Trochanova and J. D. Meister, “Three-dimensional EIT imaging of breast tissues: System design and clinical testing," IEEE Transactions on Medical Imaging, vol. 21(6), pp. 662-667, June 2002.

40 V. Cherepenin, A. Karpov, A. Korjenevsky, V. Kornienko, A. Mazaletskaya, D. Mazourov and D. Meister, “A 3D electrical impedance tomography (EIT) system for breast cancer detection,” Physiological Measurement, vol. 22(1), pp. 9-18, February 2001.

41 T. E. Kerner, K. D. Paulsen, A. Hartov, S. K. Soho and S. P. Poplack, "Electrical impedance spectroscopy of the breast: Clinical imaging results in 26 subjects," IEEE Transactions on Medical Imaging, vol. 21(6), pp. 638-645, June 2002.

42 T. E. Kerner, A. Hartov, S. K. Soho, S. P. Poplack and K. D. Paulsen, "Imaging the breast with EIS: an initial study of exam consistency,” Physiological Measurement, vol. 23(1), pp. 221-236, February 2002.

43 A. Malich, T. Boehm, M. Facius, M. Freesmeyer, T. Azhari, B. Werner, R. Anderson and W. A. Kaiser, "Electrical impedance scanning of lymph nodes: Initial clinical and technical findings,” Clinical Radiology, vol. 57(7), pp. 579-586, July 2002.

44 A. Malich, T. Boehm, M. Facius, H. J. Mentzel, M. Fleck, J. Boettcher, R. Anderson and W. A. Kaiser, "Use of electrical impedance scanning in the differentiation of sonographically suspicious and highly suspicious lymph nodes of the head-neck region,” European Radiology, vol. 12(5), pp. 1114-1120, May 2002.

45 A. Malich, T. Fritsch, C. Mauch, T. Boehm, M. Freesmeyer, M. Fleck, R. Anderson and W. A. Kaiser, "Electrical impedance scanning: a new technique in the diagnosis of lymph nodes in which malignancy is suspected on ultrasound," British Journal of Radiology, vol. 74(877), pp. 42-47, January 2001.

46 H. J. Mentzel, A. Malich, K. Kentouche, M. Freesmeyer, J. Bottcher, G. Schneider, B. Gruhn, S. Vogt, F. Zintl, R. Anderson and W. A. Kaiser, "Electrical impedance scanning - application of this new technique for lymph node evaluation in children," Pediatric Radiology, vol. 33(7), pp. 461-466, July 2003.

47 F. Y. Chang, C. L. Lu, C. Y. Chen, S. D. Lee, D. S. Tsai and S. E. Fu, “Applied potential tomography in liquid gastric emptying measurement - Design, assembling, calibration, and clinical application,” Digestive Diseases and Sciences, vol. 46(9), pp. 1839-1845, September 2001.

48 A. Giouvanoudi, W. B. Amaee, J. A. Sutton, P. Horton, R. Morton, W. Hall, L. Morgan, M. R. Freedman and N. M. Spyrou, "Physiological interpretation of electrical 
impedance epigastrography measurements,” Physiological Measurement, vol. 24(1), pp. 45-55, February 2003.

49 C. S. Chaw, E. Yazaki and D. F. Evans, "The effect of $\mathrm{pH}$ change on the gastric emptying of liquids measured by electrical impedance tomography and $\mathrm{pH}$-sensitive radiotelemetry capsule,” International Journal of Pharmaceutics, vol. 227(1-2), pp. 167175, October 2001.

50 A. Lange, P. Funch-Jensen and P. O. Schiotz, "Gastric dysrhythmia in infants with gastrointestinal diseases measured by epigastric impedance,” Journal of Pediatric Gastroenterology and Nutrition, vol. 37(3), pp. 294-299, September 2003.

51 I. Frerichs, H. Schiffmann, G. Hahn and G Hellige, "Non-invasive radiation-free monitoring of regional lung ventilation in critically ill infants," Intensive Care Medicine, vol. 27(8), pp. 1385-1394, August 2001.

52 I. Frerichs, P. A. Dargaville, T. Dudykevych and P. C. Rimensberger, "Electrical impedance tomography: a method for monitoring regional lung aeration and tidal volume distribution?” Intensive Care Medicine, vol. 29(12), pp. 2312-2316, December 2003.

53 I. Frerichs, G. Hahn, H. Schiffmann, C. Berger and G. Hellige, "Monitoring regional lung ventilation by functional electrical impedance tomography during assisted ventilation,” Annals of the New York Academy of Sciences, vol. 873, pp. 493-505, 1999.

54 I. Frerichs, W. Golisch, G. Hahn, M. Kurpitz, H. Burchardi and G. Hellige, "Heterogeneous distribution of pulmonary ventilation in intensive care patients detected by functional electrical impedance tomography,” Journal of Intensive Care Medicine, vol. 13(4), pp. 168-173, July-August 1998.

55 E. Raaijmakers, T. J. C. Faes, J. M. Meijer, P. W. A. Kunst, J. Bakker, H. G. Goovaerts and R. M. Heethaar, "Estimation of non-cardiogenic pulmonary oedema using dual-frequency electrical impedance,” Medical \& Biological Engineering \& Computing, vol. 36(4), pp. 461-466, July 1998.

56 A. V. Noordegraaf, P. W. A. Kunst, A. Janse, J. T. Marcus, P. E. Postmus, T. J. C. Faes and P. M. J. M. de Vries, "Pulmonary perfusion measured by means of electrical impedance tomography,” Physiological Measurement, vol. 19(2), pp. 263-273, May 1998.

57 P. W. A. Kunst, A. V. Noordegraaf, E. Raaijmakers, J. Bakker, A. B. J. Groeneveld, P E. Postmus and P. M. J. M. de Vries, "Electrical impedance tomography in the assessment of extravascular lung water in noncardiogenic acute respiratory failure," Chest, vol. 116(6), pp. 1695-1702, December 1999.

58 H. J. Smit, A. V. Noordegraaf, R. J. Roeleveld, J. G. F. Bronzwaer, P. E. Postmus, P. M. J. M. de Vries and A. Boonstra, "Epoprostenol-induced pulmonary vasodilatation in patients with pulmonary hypertension measured by electrical impedance tomography," Physiological Measurement, vol. 23(1), pp. 237-243, February 2002.

59 P. W. A. Kunst, S. H. Bohm, G. V. de Anda, M. B. P. Amato, B. Lachmann, P. E. Postmus and P. M. J. M. de Vries, "Regional pressure volume curves by electrical impedance tomography in a model of acute lung injury," Critical Care Medicine, vol. 28(1), pp. 178-183 January 2000.

60 T. J. Noble, N. D. Harris, A. H. Morice, P. Milnes and B. H. Brown, "Diuretic induced change in lung water assessed by electrical impedance tomography," Physiological Measurement, vol. 21(1), pp. 155-163, February 2000. 
61 R. H. Smallwood, A. R. Hampshire, B. H. Brown, R. A. Primhak, S. Marven and P. Nopp, "A comparison of neonatal and adult lung impedances derived from EIT images," Physiological Measurement, vol. 20(4), pp. 401-413, November 1999.

62 B. H. Brown, R. A. Primhak, R. H. Smallwood, P. Milnes, A. J. Narracott and M J. Jackson, "Neonatal lungs: maturational changes in lung resistivity spectra," Medical \& Biological Engineering \& Computing, vol. 40(5), pp. 506-511, September 2002.

63 A. Vonk-Noordegraaf, A. Janse, J. T. Marcus, J. G. F. Bronzwaer, P. E. Postmus, T. J. C. Faes and P. M. J. M. de Vries, "Determination of stroke volume by means of electrical impedance tomography,” Physiological Measurement, vol. 21(2), pp. 285-293, May 2000.

64 N. Polydorides, W. R. B. Lionheart and H. McCann, "Krylov subspace iterative techniques: On the detection of brain activity with electrical impedance tomography," IEEE Transactions on Medical Imaging, vol. 21(6), pp. 596-603, June 2002.

65 C. M. Towers, H. McCann, M. Wang, P. C. Beatty, C. J. D. Pomfrett and M. S. Beck, "3D simulation of EIT for monitoring impedance variations within the human head," Physiological Measurement, vol. 21(1), pp. 119-124, February 2000.

66 A. P. Bagshaw, A. D. Liston, R. H. Bayford, A. Tizzard, A. P. Gibson, A. T. Tidswell, M. K. Sparkes, H. Dehghani, C. D. Binnie and D. S. Holder, "Electrical impedance tomography of human brain function using reconstruction algorithms based on the finite element method,” Neuroimage, vol. 20(2), pp. 752-764, October 2003.

67 A. T. Tidswell, A. Gibson, R. H. Bayford and D. S. Holder, "Electrical impedance tomography of human brain activity with a two-dimensional ring of scalp electrodes," Physiological Measurement, vol. 22(1), pp. 167-175, February 2001.

68 A. Hartov, P. LePivert, N. Soni and K. Paulsen, "Using multiple-electrode impedance measurements to monitor cryosurgery,” Medical Physics, vol. 29(12), pp. 2806-2814, December 2002.

69 M. M. Radai, S. Abboud and B. Rubinsky, "Evaluation of the impedance technique for cryosurgery in a theoretical model of the head," Cryobiology, vol. 38(1), pp. 51-59, February 1999.

70 S. Zlochiver, M. M. Radai, M. Rosenfeld and S. Abboud, "Induced current impedance technique for monitoring brain cryosurgery in a two-dimensional model of the head," Annals of Biomedical Engineering, vol. 30(9), pp. 1172-1180, October 2002.

71 Imaginis, The Breast Health Resource, "Breast Cancer: Statistics on Incidence, Survival, and Screening”, http://imaginis.com/breasthealth/statistics.asp, April, 2005.

72 J. P. Morucci and P. M. Marsili, "Bioelectrical impedance techniques in medicine .3. Impedance imaging - Second section: Reconstruction algorithms," Critical Reviews in Biomedical Engineering, vol. 24(4-6), pp. 599-654, 1996.

73 R. Plonsey and D. B. Heppner, "Considerations of Quasi-Stationarity in Electrophysiological Systems,” Bulletin of Mathematical Biophysics, vol. 29, pp. 657664, 1967.

74 J. D. Jackson, Classical electrodynamics, $2^{\text {nd }}$ edition, New York : Wiley, 1975.

75 R. P. Henderson and J. G. Webster, "An Impedance Camera for Spatially Specific Measurements of the Thorax," IEEE Transactions on Biomedical Engineering, vol. 25, pp. 250 - 254, 1978. 
76 D. C. Barber and B. H. Brown "Applied Potential Tomography,” Journal of Physics E: Scientific. Instrumentation, vol. 17, pp. 723 - 733, 1984.

77 T. Murai and Y. Kagawa, "Electrical impedance computed tomography based on a finiteelement model,” IEEE Transactions on Biomedical Engineering, vol. 32, pp. 177-184, 1985.

78 D. B. Geselowitz, "An application of electrocardiographic lead theory to impedance plethsmography,” IEEE Transactions on Biomedical Engineering, vol. 18, pp. 38-41, 1971.

79 Y. Kim, J. G. Webster and W. J. Tompkins, "Electrical impedance imaging of the Thorax,” Journal of Microwave Power, vol. 18, pp. 245-257, 1983.

80 P. Gilbert, "Iterative methods for the reconstruction of three-dimensional objects from projections," Journal of Theoretical Biology, vol. 36, pp. 105-117, 1972.

81 T. J. Yorkey, J. G. Webster and W. J. Tompkin, "An optimal impedance tomographic reconstruction algorithm," in Proceedings of the $8^{\text {th }}$ Annual Conference of the IEEE Engineering Medical and Biological Society, pp. 339-342, 1986.

82 A. Wexler, B. Fry and M. R. Neuman, "Impedance-computed tomography algorithm and system,” Applied Optics, vol. 24, pp. 3985-3992, 1985.

83 P. Tong and J. N. Rossettos, Finite-element Method: Basic Technique and Implementation, Cambridge MA: MIT Press, 1977.

84 T. J. Yorkey, J. G. Webster and W. J. Tompkins, "Comparing reconstruction algorithms for electrical-impedance tomography,” IEEE Transactions on Biomedical Engineering, vol. 34(11), pp. 843-852, November 1987.

85 D. C. Barber, "A review of image-reconstruction techniques for electrical-impedance tomography,” Medical Physics, vol. 16(2), pp. 162-169, March-April 1989.

86 K. Boone, D. Barber and B. Brown, "Imaging with electricity: Report of the European Concerted Action on Impedance Tomography," Journal of Medical Engineering \& Technology, vol. 21(6), pp. 201-232, November-December 1997.

87 M. Cheney, D. Isaacson and J. C. Newell, "Electrical impedance tomography," SIAM Review, vol. 41(1), pp. 85-101, March 1999.

88 D. C. Barber, B. H. Brown and N. J. Avis, "Image Reconstruction in Electrical Impedance Tomography Using Filtered Back-Projection,” in Proceedings of the Annual International Conference of the IEEE Engineering in Medicine and Biology Society, vol. 14, pp. 1691 - 1692, 1992.

89 F. Santosa and M. Vogelius, "A backprojection algorithm for electrical-impedance imaging,” SIAM Journal on Applied Mathematics, vol. 50(1), pp. 216-243, February 1990.

90 R. Bayford, Y. Hanquan, K. Boone and D. S. Holder, "Experimental validation of a novel reconstruction algorithm for electrical-impedance tomography based on backprojection of Lagrange multipliers," Physiological Measurement, vol. 16, pp. A237A247, August 1995.

91 R. Guardo, C. Boulay, B. Murray and M. Bertrand, "An experimental-study in electricalimpedance tomography using backprojection reconstruction,” IEEE Transactions on Biomedical Engineering, vol. 38(7), pp. 617-627, July 1991. 
92 A. F. Frangi, P. J. Riu, J. Rosell and M. A. Viergever, "Propagation of measurement noise through backprojection reconstruction in electrical impedance tomography," IEEE Transactions on Medical Imaging, vol. 21(6), pp. 566-578, June 2002.

93 J. P. Morucci, P. M. Marsili, M. Granie, Y. Shi, M. Lei and W. W. Dai, "A direct sensitivity matrix approach for fast reconstruction in electrical-impedance tomography," Physiological Measurement, vol. 15, pp. A107-A114, May 1994.

94 J. P. Morucci, M. Granie, M. Lei, M. Chabert and P. M. Marsili, "3D reconstruction in electrical-impedance imaging using a direct sensitivity matrix approach,” Physiological Measurement, vol. 16, pp. A123-A128, August 1995.

95 E. Somersalo, M. Cheney, D. Isaacson and E. Isaacson, "Layer stripping - a direct numerical-method for impedance imaging, inverse problems,” vol. 7(6), pp. 899-926 December 1991.

96 M. Cheney, D. Isaacson, J. C. Newell, S. Simske and J. Goble, "NOSER: An algorithm for solving the inverse conductivity problem," International Journal of Imaging Systems and Technology, pp 66-75, 1990.

97 P. M. Edic, G. J. Saulnier, J. C. Newell and D. Isaacson, “A real-time electricalimpedance tomograph,” IEEE Transactions on Biomedical Engineering, vol. 42(9), pp. 849-859 September 1995.

98 A. Le Hyaric and M. K. Pidcock, "A one step image reconstruction algorithm for electrical impedance tomography in three dimensions," Physiological Measurement, vol. 21(1), pp. 95-98, February 2000.

99 A. Le Hyaric and M. K. Pidcock, "An image reconstruction algorithm for threedimensional electrical impedance tomography," IEEE Transactions on Biomedical Engineering, vol. 48(2), pp. 230-235, February 2001.

100 S. Siltanen, J. Mueller and D. Isaacson, "An implementation of the reconstruction algorithm of A Nachman for the 2D inverse conductivity problem,” Inverse Problems, vol. 16(3), pp. 681-699, June 2000.

101 J. L. Mueller, S. Siltanen and D. Isaacson, "A direct reconstruction algorithm for electrical impedance tomography,” IEEE Transactions on Medical Imaging, vol. 21(6), pp. 555-559, June 2002.

102 J. L. Mueller and S. Siltanen, "Direct reconstructions of conductivities from boundary measurements," SIAM Journal on Scientific Computing, vol. 24(4), pp. 1232-1266, 2003.

103 J. E. Akin, Finite element analysis with error estimators: An introduction to the FEM and adaptive error analysis for engineering students, Burlington, MA: Elsevier/ButterworthHeinemann, 2005.

104 A. R. Mitchell, The finite difference method in partial differential equations, New York: Wiley, 1980.

105 O. P. Gupta, Finite and boundary element methods in engineering, Brookfield, VT: A.A. Balkema, 1999.

106 U.S. National Library of Medicine, National Institutes of Health, "The Visible Human Project®," September 2003, http://www.nlm.nih.gov/research/visible/visible_human.html.

107 Y. Z. Ider, S. Onart and W. R. B. Lionheart, "Uniqueness and reconstruction in magnetic resonance-electrical impedance tomography (MR-EIT)," Physiological Measurement, vol. 24(2), pp. 591-604, May 2003. 
108 X. L. Zhao, Y. Kinouchi, T. Iritani, T. Morimoto and M. Takeuchi, "Estimation of multi-layer tissue conductivities from non-invasively measured bioresistances using divided electrodes,” IEICE Transactions on Information and Systems, vol. E85D(6), pp. 1031-1038, June 2002.

109 X. L. Zhao, Y. Kinouchi, E. Yasuno, D. Gao, T. Iritani, T. Morimoto and A. Takeuchi, "A new method for noninvasive measurement of multilayer tissue conductivity and structure using divided electrodes,” IEEE Transactions on Biomedical engineering, vol. 51(2), pp. 362-370, February 2004.

110 R. P. Patterson and J. Zhang, "Evaluation of an EIT reconstruction algorithm using finite difference human thorax models as phantoms," Physiological Measurement, vol. 24(2), pp. 467-475, May 2003.

111 R. H. Bayford, A. Gibson, A. Tizzard, T. Tidswell and D. S. Holder, "Solving the forward problem in electrical impedance tomography for the human head using IDEAS (integrated design engineering analysis software), a finite element modeling tool," Physiological Measurement, vol. 22(1), pp. 55-64, February 2001.

112 A. Borsic, C. McLeod, W. Lionheart and N. Kerrouche, "Realistic 2D human thorax modeling for EIT,” Physiological Measurement, vol. 22(1), pp. 77-83, February 2001.

113 P. M. Marsili, G. Mounie, M. Granie and J. P. Morucci, "Conductivity interface modeling with dipoles by means of optimal-control and boundary element methods in impedance tomography,” Clinical Physics and Physiological Measurement, vol. 13, pp. 107-111, 1992.

114 Y Shi, P. M. Marsili, W. W. Dai, M. Granie and J. P. Morucci, “Application of the boundary element method to the study of boundary potentials in electrical-impedance tomography," Clinical Physics and Physiological Measurement, vol. 13, pp. 139-143, 1992.

115 J. C. de Munck, T. J. C. Faes, A. J. Hermans and R. M. Heethaar, “A parametric method to resolve the ill-posed nature of the EIT reconstruction problem - A simulation study,” Electrical Bioimpedance Methods: Applications to Medicine and Biotechnology, vol. 873, pp. 440-453, 1999.

116 J. C. de Munck, T. J. C. Faes and R. M. Heethaar, "The boundary element method in the forward and inverse problem of electrical impedance tomography,” IEEE Transactions on Biomedical Engineering, vol. 47(6), pp. 792-800, June 2000.

117 S. I. Goncalves, J. C. de Munck, J. P. A. Verbunt, F. Bijma, R. M. Heethaar and F. L. da Silva, "In vivo measurement of the brain and skull resistivities using an EIT-based method and realistic models for the head,” IEEE Transactions on Biomedical Engineering, vol. 50(6), pp. 754-767 June 2003.

118 H. Jain, D. Isaacson, P. M. Edic, J. C. Newell, "Electrical impedance tomography of complex conductivity distributions with noncircular boundary,” IEEE Transactions on Biomedical Engineering, vol. 44(11), pp. 1051-1060, November 1997.

119 R. Duraiswami, K. Sarkar and G. L. Chahine, "Efficient 2D and 3D electrical impedance tomography using dual reciprocity boundary element techniques,” Engineering Analysis with Boundary Elements, vol. 22(1), pp. 13-31, July 1998.

120 M. Tarvainen, M. Vauhkonen, T. Savolainen and J. P. Kaipio, "Boundary element method and internal electrodes in electrical impedance tomography,” International Journal for Numerical Methods in Engineering, vol. 50(4), pp. 809-824, February 2001. 
121 F. Kleinermann, N. J. Avis, S. K. Judah and D. C. Barber, "Three-dimensional image reconstruction for electrical impedance tomography,” Physiological Measurement, vol. 17, pp. A77-A83, November 1996.

122 M. T. Clay and T. C. Ferree, "Weighted regularization in electrical impedance tomography with applications to acute cerebral stroke,” IEEE Transactions on Medical Imaging, vol. 21(6), pp. 629-637, June 2002.

123 R. Gadd, F. Vinther, P. M. Record and P. Rolfe, "Reconstruction of 3-dimensional data for electrical-impedance tomography,” Electronics Letters, vol. 28(11), pp. 974-976, May 1992.

124 C. J. Kotre, "EIT image-reconstruction using sensitivity weighted filtered backprojection,” Physiological Measurement, vol. 15, pp. A125-A136, May 1994.

125 R. Gadd, P. M. Record and P. Rolfe, "On the sensitivity method of reconstruction for electrical-impedance tomography,” Clinical Physics and Physiological Measurement, vol. 11(2), pp. 180-181, May 1990.

126 K. Jerbi, W. R. B. Lionheart, P. J. Vauhkonen and M. Vauhkonen, "Sensitivity matrix and reconstruction algorithm for EIT assuming axial uniformity,” Physiological Measurement, vol. 21(1), pp. 61-66, February 2000.

127 E. Ratajewicz-Mikolajczak, G. H. Shirkoohi and J. Sikora, "Two ANN reconstruction methods for electrical impedance tomography,” IEEE Transactions on Magnetics, vol. 34(5), pp. 2964-2967, September 1998.

128 A. Adler and R. Guardo, "A neural-network image-reconstruction technique for electricalimpedance tomography,” IEEE Transactions on Medical Imaging, vol. 13(4), pp. 594600, December 1994.

129 E. Ratajewicz-Mikolajczak and J. Sikora, "Neural networks method for identification of the objects behind the screen,” IEEE Transactions on Medical Imaging, vol. 21(6), pp. 613-619, June 2002.

130 A. S. Miller, B. H. Blott and T. I. Hames, "Neural networks for electrical-impedance tomography image characterization,” Clinical Physics and Physiological Measurement, vol. 13, pp. 119-123, 1992.

131 A. I. Nachman, "Global Uniqueness for a Two-Dimensional Inverse Boundary Value Problem,” Annals of Mathematics, vol. 143(1), pp. 71-96, January 1996.

132 G. Allesandrini, "Stable Determination of Conductivity by Boundary Measurements," Applied Analysis, vol. 27, pp. 153-172, 1988.

133 Institut für Numerische und instrumentelle Mathematik, Universität Münster, “Algorithms in Tomography," June 1996, http://wwwmath.unimuenster.de/math/inst/num/Preprints/1997/natterer_4/l2html/node2.html

134 E. J. Woo, P. Hua, J. G. Webster and W. J. Tompkins, “A robust image-reconstruction algorithm and its parallel implementation in electrical-impedance tomography,” IEEE Transactions on Medical Imaging, vol. 12(2), pp. 137-146, June 1993.

135 J Artola, J. Dell, "Broyden quasi-newton method applied to electrical-impedance tomography,” Electronics Letters, vol. 30(1), pp. 27-28, January 1994.

136 W. W. Loh and F. J. Dickin, "Improved modified Newton-Raphson algorithm for electrical impedance tomography,” Electronics Letters, vol. 32(3), pp. 206-207, February 1996. 
137 C. J. Grootveld, A. Segal and B. Scarlett, "Regularized modified Newton-Raphson technique applied to electrical impedance tomography,” International Journal of Imaging Systems and Technology, vol. 9(1), pp. 60-65, 1998.

138 P. M. Edic, D. Isaacson, G. J. Saulnier, H. Jain and J. C. Newell, “An iterative Newton-Raphson method to solve the inverse admittivity problem," IEEE Transactions on Biomedical Engineering, vol. 45(7), pp. 899-908, July 1998.

139 L. Y. Rao, R. J. He, Y. H. Wang, W. L. Yan, J. Bai and D. T. Ye, “An efficient improvement of modified Newton-Raphson algorithm for electrical impedance tomography,” IEEE Transactions on Magnetics, vol. 35(3), pp. 1562-1565, May 1999.

140 M. A. Player, J. van Weereld, A. R. Allen, D. A. L. Collie, "Truncated-Newton algorithm for three-dimensional electrical impedance tomography,” Electronics Letters, vol. 35(25), pp. 2189-2191, December 1999.

141 M. Glidewell and K. T. Ng, “Anatomically constrained electrical-impedance tomography for anisotropic bodies via a 2-step approach,” IEEE Transactions on Medical Imaging, vol. 14(3), pp. 498-503, September 1995.

142 M. E. Glidewell and K.T. Ng, "Anatomically constrained electrical impedance tomography for three-dimensional anisotropic bodies,” IEEE Transactions on Medical Imaging, vol. 16(5), pp. 572-580, October 1997.

143 U. Baysal and B. M. Eyuboglu, "Use of a priori information in estimating tissue resistivities - a simulation study," Physics in Medicine and Biology, vol. 43(12), pp. 35893606, December 1998.

144 U. Baysal and B. M. Eyuboglu, "Use of a priori information in estimating tissue resistivities-application to measured data,” Physics in Medicine and Biology, vol. 44(7), pp. 1677-1689, July 1999.

145 U. Baysal and B.M. Eyuboglu, "Tissue resistivity estimation in the presence of positional and geometrical uncertainties,” Physics in Medicine and Biology, vol. 45(8), pp. 23732388, August 2000.

146 M. Vauhkonen, J. P. Kaipio, E. Somersalo and P. A. Karjalainen, "Electrical impedance tomography with basis constraints," Inverse Problems, vol. 13(2), pp. 523-530, April 1997.

147 J. P. Kaipio, V. Kolehmainen, M. Vauhkonen and E. Somersalo, "Inverse problems with structural prior information,” Inverse Problems, vol. 15(3), pp. 713-729, June 1999.

148 Wikipedia, the free encyclopedia, "Sigmoid function,” October 2005, http://en.wikipedia.org/wiki/Sigmoid_function

149 W. H. Press, S. A. Teukolsky, W. T. Vetterling, and B. P. Flannery, Numerical Recipes in FORTRAN: The Art of Scientific Computing, Second Edition, New York: Cambridge University Press, 1992.

150 The American Heritage ${ }^{\circledR}$ Stedman's Medical Dictionary, Houghton Mifflin Company, 2002.

1511260 Impedance Gain-Phase Analyzer Operating Manual, Solartron Analytical, December 1995.

152 ZPlot ${ }^{\circledR}$ for Windows, Scribner and Associates, 1998.

153 B. Blad, "An electrical impedance tomography system for complex impedance imaging," Innovation et Technologie en Biologie et Medecine, vol. 15(No. Special 1), pp. 80-88, 1994. 
154 B. Blad, K. Lindstroem, L. Bertenstam, B. R. R. Persson and H.-G. Holmer, “A current injecting device for electrical impedance tomography,” Physiological Measurement, vol. 15(Suppl. 2A), pp. A69-A77, 1994.

155 A. Hartov, R. A. Mazzarese, F. R. Reiss, T. E. Kerner, K. S. Osterman,D. B. Williams and K. D. Paulsen, A multichannel continuously selectable multifrequency electrical impedance spectroscopy measurement system, IEEE Transactions on Biomedical Engineering, vol. 47(1), pp. 49 - 58, January 2000.

156 M. R. Spiegle, editor, Theory and Problems of Statistics, $2^{\text {nd }}$ ed, Schaum's Outline Series, New York: McGraw-Hill, 1994.

157 Quality America Inc., "Coefficient of Determination R Squared,” http://www.qualityamerica.com/knowledgecente/knowctrCoefficient_of_Determination_R S.htm

158 G. Kehrer, A. Aminalai, E. Burger, E. Gersing, F. Grossmann, A. Mari, P. A. Schnabel, M. Wagner, J. Richter, H. Bosseckert and H. J. Bretschneider, "Interrelations between electrical and biochemical processes in ischemic porcine livers at lowtemperature,” Zeitschrift fur Gastroenterologie, vol. 31(3), pp. 171-178, March 1993. 